\title{
Aufbau von Bausteinen zur Synthese von Spinosynanaloga
}

\author{
Dissertation \\ zur Erlangung des Doktorgrades \\ „Doctor rerum naturalium" \\ der Mathematisch-Naturwissenschaftlichen Fakultäten \\ der Georg-August-Universität zu Göttingen
}

vorgelegt von
Timo Scheffer
aus Bad Hersfeld

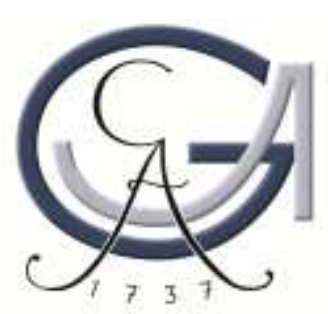

Göttingen 2012 

D7

Referent:

Prof. Dr. Dr. h.c. Lutz F. Tietze

Korreferent:

Prof. Dr. Hartmut Laatsch

Tag der mündlichen Prüfung: $\quad$ 21.02.2012 

Die vorliegende Arbeit wurde in der Zeit von September 2008 bis Januar 2012 am Institut für Organische und Biomolekulare Chemie der Georg-August-Universität Göttingen unter der Leitung von Prof. Dr. Dr. h.c. Lutz F. Tietze angefertigt.

Mein besonderer Dank gilt Herrn Prof. Dr. Dr. h.c. Lutz F. Tietze für die interessante Themenstellung, sein stetes Interesse am Fortgang dieser Arbeit sowie für die zahlreichen Diskussionen und Anregungen. 

Meiner FAmilie,

MeINEN FREUnden 

„Auch eine Enttäuschung, wenn sie nur gründlich und endgültig ist, bedeutet einen Schritt vorwärts.“

Max Planck 



\section{A. INHALTSVERZEICHNIS}

A.INHALTSVERZEICHNIS I

B.ALLGEMEINER TEIL .............................................................................. 1

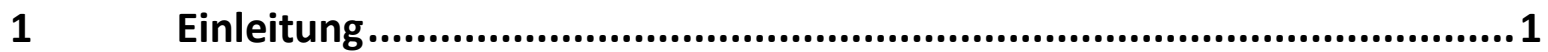

2 Der moderne Pflanzenschutz ............................................................

2.1 Die Geschichte des Pflanzenschutzes..................................................

2.2 Anforderungen an neue Wirkstoffe .................................................. 6

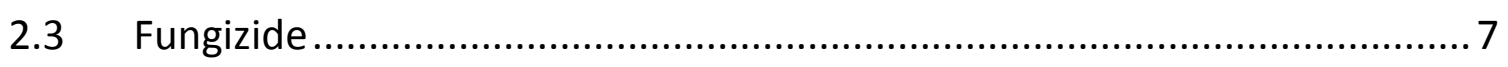

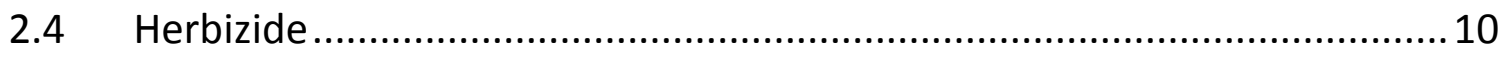

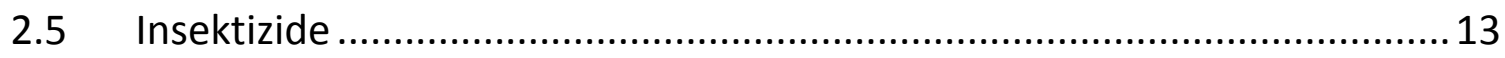

3 Die Naturstoffklasse der Spinosyne .......................................... 18

3.1 Entdeckung, Identifizierung und Strukturmerkmale ............................. 18

3.2 Eigenschaften und Wirkungsweise................................................... 19

S.3 Stand der gegenwärtigen Forschung............................................. 23

3.3.1 Naturstoffderivatisierung - Struktur-Aktivitäts-Beziehungen und Synthese von Spinosoiden ..................................................... 23

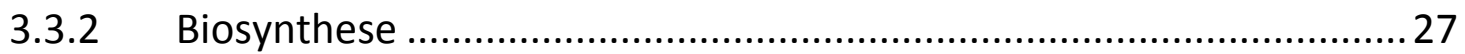

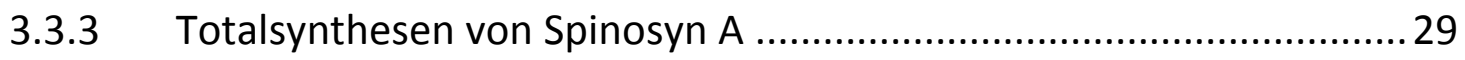

C. ZIELSETZUNG UND PLANUNG DER ARBEIT.......................................... 33

D.DARSTELLUNG DER ERGEBNISSE .............................................. 37

1 Synthese des cis-disubstituierten Cyclopentenbausteins rac-69............37

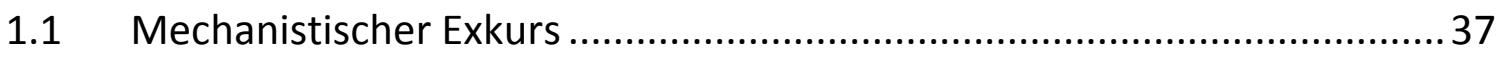

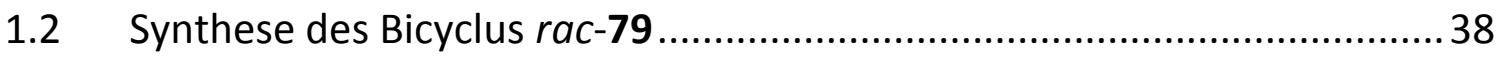

1.3 Synthese des Cyclopentens rac-69 ................................................ 40

1.4 Diskussion ausgewählter spektroskopischer Daten der Verbindung rac-69 
$2 \quad$ Synthese des aromatischen Bausteins 62 45

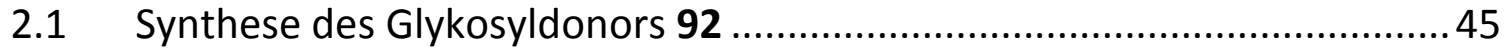

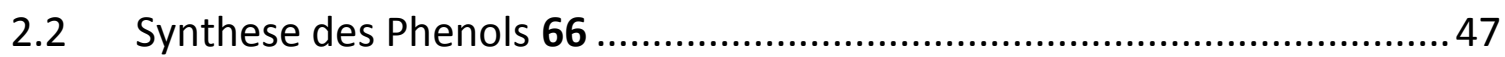

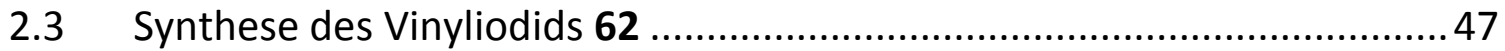

2.4 Diskussion ausgewählter spektroskopischer Daten der Verbindung $62 . . .48$ Synthese des Tricyclus 60 .........................................................5 51

3.1 Inter- und Intramolekulare Heck-Reaktion .........................................51

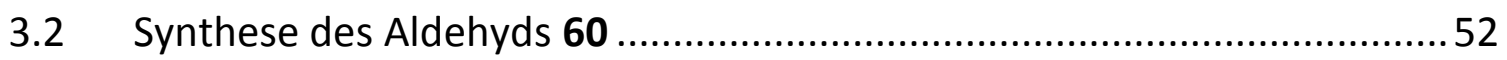

3.3 Diskussion ausgewählter spektroskopischer Daten der Verbindung $60 \ldots 53$

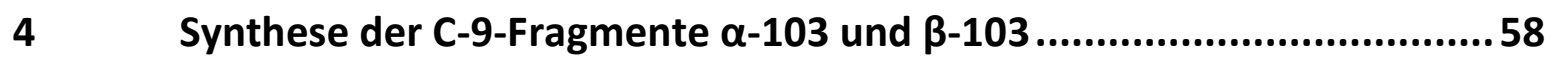

4.1 Synthese der C-6-Bausteine $\alpha-115$ und $\beta-115$......................................58

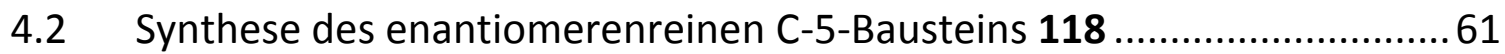

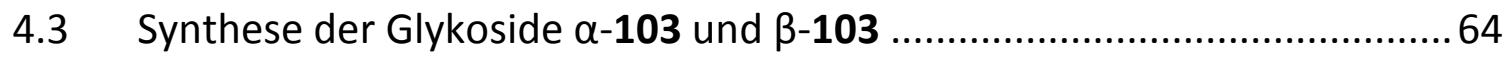

4.4 Diskussion ausgewählter spektroskopischer Daten der Verbindung $\beta-103$ 68

$5 \quad$ Untersuchungen zur Kupplung des Tricyclus 60 mit den

Bausteinen $\alpha / \beta-103$................................................................ 72

5.1 Untersuchungen zur 1,2-Addition .................................................. 72

5.2 Untersuchungen zur Umpolung des Aldehyds 60 .............................. 75

E. ZUSAMMENFASSUNG .......................................................... 79

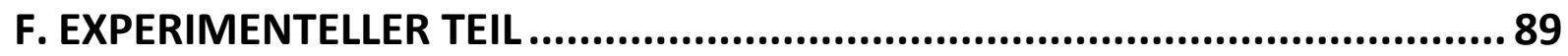

$1 \quad$ Allgemeine Arbeitstechniken .......................................................89

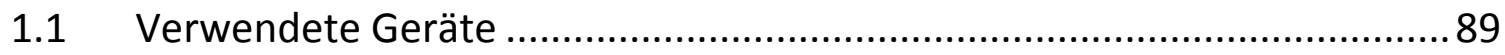

1.2 Chromatographische Methoden ........................................................ 91

2 Synthese der verwendeten Reagenzien ......................................93

$2.1 \quad N, N^{\prime}$-Di-iso-propyl-O-tert-butyl-iso-harnstoff (87) .................................93

2.2 Polymer-gebundenes 1,8-Diazabicyclo[5.4.0]undec-7-en (93) ................ 93

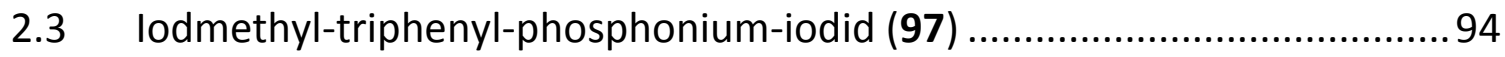


3 Synthese des cis-disubstituierten Cyclopentenderivats 69 96

3.1 Malonsäuremonomethylester (74) ................................................ 96

3.2 rac-2-Chlor-2-chlorcarbonyl-essigsäuremethylester (75) ......................97

3.3 rac-(1R,5S)-6-Chlor-7-Oxobicyclo[3.2.0]hept-3-en-6-

carbonsäuremethylester (78)

3.4 rac-(1R,5R,6R/S)-7-Oxobicyclo[3.2.0]hept-3-en-6-

carbonsäuremethylester (79) 98

3.5 rac-(4aS,7aR)-4,4a,7,7a-Tetrahydro-1H-cyclopenta[c]pyran-3-on (83).....99

3.6 rac-(1S,5R)-2-[(5-Hydroxymethyl)-cyclopent-2-enyl]-essigsäure-tertbutylester (85) 100

3.7 rac-(1S,5R)-2-[(5-tert-Butyldimethylsilyloxymethyl)-

cyclopent-2-enyl]essigsäure-tert-butylester (69) 102

4 Synthese des aromatischen Fragments 62 103

4.1 Methyl- $\alpha$-L-rhamnopyranosid (89) 103

4.2 1,2,3,4-Tetra-O-methyl- $\alpha$-L-rhamnopyranosid (90) ............................. 103

$4.3 \quad 2,3,4-T r i-O-m e t h y l-\alpha / \beta$-L-rhamnopyranosid (91) ................................. 104

$4.4 \quad 0$-(2,3,4-Tri-O-methyl- $\alpha$-L-rhamnopyranosyl)trichloracetimidat (92) .....105

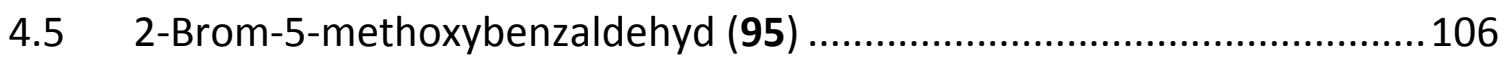

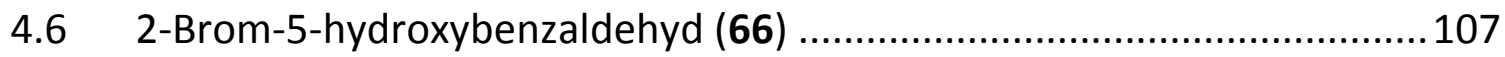

4.7 2-Brom-5-(2,3,4-tri-O-methyl- $\alpha$-L-rhamnopyranosyl)benzaldehyd (96).. 108

4.8 (Z)-2-(2-lodethenyl)-4-(2,3,4-tri-O-methyl- $\alpha$-L-rhamnopyranosyl)brombenzol (62) 109

$5 \quad$ Synthese des Tricyclus 102 111

$5.12-\{2-[(Z)-2-(2-B r o m-5-(2,3,4-t r i-O-m e t h y l-\alpha-L-r h a m n o p y r a n o s y l)-$ phenyl)-vinyl]-5-tert-butyldimethylsilyloxymethyl-cyclopent-3-enyl\}essigsäure-tert-butylester (98)

5.2 2-[2-(tert-Butyldimethylsilyloxymethyl)-7-(2,3,4-tri-O-methyl- $\alpha$-Lrhamnopyranosyl)-3a,9b-dihydro-3H-cyclopenta[a]naphthalin-3-yl]essigsäure-tert-butylester (100) .112

5.3 2-[2-Hydroxymethyl-8-(2,3,4-tri-O-methyl- $\alpha$-I-rhamnopyranosyl)- 
3a,9b-dihydro-3H-cyclopenta[a]naphthalin-3-yl]-essigsäure-

tert-butylester (102) 114

5.4 Diastereomerentrennung von 2-[2-Hydroxymethyl-8-

(2,3,4-tri-O-methyl- $\alpha$-L-rhamnopyranosyl)-3a,9b-dihydro-3H-

cyclopenta[a]naphthalin-3-yl]-essigsäure-tert-butylester (102) 114

5.5 (3S,3aS,9bS)-2-(2-Formyl-7-(2,3,4-tri-O-methyl- $\alpha$-L-rhamnopyranosyl)3a,9b-dihydro-3H-cyclopenta[a]naphthalin-3-yl)-essigsäure-

tert-butylester (60) 116

6 Synthese des C-9 Fragments 103 118

6.1 (S)-2-(Benzyloxy)propansäuremethylester (105) ................................ 118

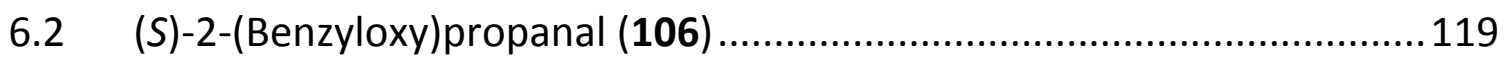

6.3 (2S,3S)-2-(Benzyloxy)hex-5-en-3-ol (107) ........................................... 120

$6.4 \quad(\mathrm{rac})$-Pent-1-en-3-yl-3,5-dinitrobenzoat (118) .................................... 121

6.5 (S)-Pent-1-en-3-yl-3,5-dinitrobenzoat (118) ..................................... 122

6.6 HPLC-Reinigung von (S)-Pent-1-en-3-yl-3,5-dinitrobenzoat (118) ...........123

6.7 (2S,3S,7S)-2-(Benzyloxy)-3-(N,N-didemethyl-N-benzyloxycarbonyl$\alpha$-D-forosaminyl)-non-5-en-7-yl-3,5-dinitrobenzoat ( $\alpha$-119) .

6.8 (2S,3S,7S)-2-(Benzyloxy)-3-(N,N-didemethyl-N-benzyloxycarbonyl-

$\beta$-D-forosaminyl)-non-5-en-7-yl-3,5-dinitrobenzoat ( $\beta$-119)

6.9 (2S,3S,7S)-2-(Benzyloxy)-3-( $N, N$-didemethyl- $N$-benzyloxycarbonyl-

$\alpha$-D-forosaminyl)-non-5-en-7-ol ( $\alpha$-120) 126

6.10 (2S,3S,7S)-2-(Benzyloxy)-3-(N,N-didemethyl-N-benzyloxycarbonyl-

$\beta$-D-forosaminyl)-non-5-en-7-ol ( $\beta$-120)

6.11 (2S,3S,7S)-2-(Benzyloxy)-7-((tert-butyldimethylsilyl)oxy)-3-(N,N-

didemethyl- $N$-benzyloxycarbonyl- $\alpha$-D-forosaminyl)-non-5-en ( $\alpha-121)$...129

6.12 (2S,3S,7S)-2-(Benzyloxy)-7-((tert-butyldimethylsilyl)oxy)-3-(N,N-

didemethyl- $N$-benzyloxycarbonyl- $\beta$-D-forosaminyl)-non-5-en ( $\beta$-121) ... 130

$6.13(2 S, 3 S, 7 S)-2-($ Benzyloxy)-7-((tert-butyldimethylsilyl)oxy)-3-(N,N-

didemethyl- $N$-benzyloxycarbonyl- $\alpha$-D-forosaminyl)-nonan ( $\alpha-122)$.......132

6.14 HPLC-Reinigung von (2S,3S,7S)-2-(Benzyloxy)-7- 
((tert-butyldimethylsilyl)oxy)-3-(N,N-didemethyl-

$N$-benzyloxycarbonyl- $\alpha$-D-forosaminyl)-nonan ( $\alpha$-122)

6.15 (2S,3S,7S)-2-(Benzyloxy)-7-((tert-butyldimethylsilyl)oxy)-3-(N,N-

didemethyl- $N$-benzyloxycarbonyl- $\beta$-D-forosaminyl)-nonan ( $\beta$-122) ........134

6.16 HPLC-Reinigung von (2S,3S,7S)-2-(Benzyloxy)-7-

((tert-butyldimethylsilyl)oxy)-3-(N,N-didemethyl-

$N$-benzyloxycarbonyl- $\beta$-D-forosaminyl)-nonan ( $\beta$-122).

6.17 (2S,3S,7S)-2-(Benzyloxy)-7-((tert-butyldimethylsilyl)oxy)-

3-( $\alpha$-D-forosaminyl)-nonan ( $\alpha-123)$

6.18 (2S,3S,7S)-2-(Benzyloxy)-7-((tert-butyldimethylsilyl)oxy)-

3-( $\beta$-D-forosaminyl)-nonan ( $\beta$-123)

6.19 (2S,3S,7S)-7-((tert-Butyldimethylsilyl)oxy)-3-( $\alpha$-D-forosaminyl)-

nonan-2-ol ( $\alpha$-103)

6.20 (2S,3S,7S)-7-((tert-Butyldimethylsilyl)oxy)-3-( 3 -D-forosaminyl)-

nonan-2-ol $(\beta-103)$ 140

G.ANHANG 





\section{B. Allgemeiner TeIL}

\section{$1 \quad$ Einleitung}

Im Herbst des Jahres 2011 wurde die symbolische Geburt des sieben Milliardsten Menschen gefeiert. Doch gleichzeitig löst das Überschreiten dieser Sieben-MilliardenMarke besonders in den ärmeren Teilen der Erde große Ängste aus. Die starke Bevölkerungszunahme bringt enorme Herausforderungen mit sich; so wird der Druck auf die Gesundheits- und Bildungssysteme sowie auf die Ernährungslage weiter steigen. Wie sollen immer mehr Menschen ernährt werden? Schon heute leiden Schätzungen der Weltgesundheitsorganisation WHO zufolge etwa eine Milliarde Menschen an ständigem Hunger. Unterdessen wird in den reichen Ländern Getreide an Vieh verfüttert sowie zur Deckung des Energiehaushalts verwendet.

Während die Weltbevölkerung weiter wächst, hat aber die Zunahme der Flächen für die Nahrungsmittelproduktion Grenzen, denn die Erde wird nicht größer. Es ist also aufgrund der immer kleiner werdenden Anbauflächen notwendig, die Leistungen der Kulturpflanzen zu erhöhen. Dafür hat die Chemie der Landwirtschaft zwei äußerst hilfreiche Mittel zur Seite gestellt: den Mineraldünger und die Pflanzenschutzmittel. Besonders die Entwicklung im Bereich Pflanzenschutzmittel ist dabei in den letzten beiden Jahrzehnten erstaunlich. Getrieben von ständig erhöhten Auflagebedingungen seitens der Gesetzgeber hat sich die agrochemische Branche fast ungeachtet von der Öffentlichkeit zu einer High-Tech-Industrie entwickelt und ist in Sachen Wissenschaftlichkeit und Qualitätsansprüchen durchaus mit der Pharmaindustrie zu vergleichen. Dass dieser Vergleich durchaus sinnvoll ist, zeigt sich auch bei der Überlegung, welche Bedeutung Pflanzenschutzmittel für Pflanzen besitzen. Sie sollen, wie Medikamente für Menschen und Tiere, die Pflanzen von Krankheiten heilen oder sie vor Angriffen von Fremdorganismen schützen. Auch das Thema Resistenzen spielt im Bereich Pflanzenschutz eine immer größere Rolle. Während die Pharmaindustrie gegen multiresistente Bakterienstämme kämpft und auf der Suche nach neuen Antibiotika 
ist, bekommt es die agrochemische Industrie vor allem im Bereich der Insektizide und Herbizide mit Resistenzen zu tun.

Darüber hinaus hat besonders der Aspekt der Umweltverträglichkeit in den letzten Jahren stark an Bedeutung gewonnen. Die Landwirtschaft war und ist immer noch einer der wichtigsten Nutzer unserer Umwelt. Die Forderungen im Rahmen des integrierten Pflanzenbaus zeigen deutlich, dass die Landwirtschaft künftig noch naturund umweltverträglicher gestaltet werden soll.

Dies alles zeigt wie wichtig es ist, neue Wirkstoffe zu entwickeln. Dabei sind, wie bereits beschrieben, die Anforderungen an einen modernen Wirkstoff sehr vielfältig. Glücklicherweise stellt die Natur eine Vielzahl von hochpotenten Verbindungen bereit, die, aus Pflanzen, Pilzen, marinen Organismen oder Mikroorganismen extrahiert, als Leitstrukturen für innovative Pflanzenschutzmittel dienen können. Die direkte Gewinnung von Wirkstoffen aus den natürlichen Quellen ist aber häufig sehr aufwendig und kann auch nicht immer die benötigten Wirkstoffmengen abdecken. Eine wichtige Rolle für die Bewältigung dieser Probleme könnte der Synthesechemie zukommen. Unter Verwendung neuer effizienter Synthesekonzepte ist es möglich, nicht nur die Wirkstoffe "nachzubauen“, sondern auch Derivate zu synthetisieren, die ein erweitertes oder verbessertes Wirkprofil aufweisen.

Die vorliegende Arbeit beschäftigt sich mit der Entwicklung von Bausteinen für eine neue konvergente Syntheseroute zum Aufbau eines Spinosynanalogons. Die Spinosyne sind natürlich vorkommende Verbindungen, die eine hohe insektizide Wirkung gepaart mit einem außergewöhnlich guten ökologischen Profil aufweisen. Es wurden jedoch in Labor- und Feldstudien erste Resistenzen beobachtet, welche die Entwicklung neuer Analoga dringend erforderlich machen. 


\section{Der moderne Pflanzenschutz}

Die Flächenerträge der wichtigen Nahrungspflanzen dieser Erde haben in den vergangenen Jahrhunderten eine Aufsehen erregende Steigerung vollzogen. Ohne diese Entwicklung wäre weder eine ausreichende Ernährung eines Großteils der immer größer werdenden Weltbevölkerung noch der rasch wachsende Wohlstand in den Industrieländern möglich gewesen. Hierzu haben vor allem neue, äußerst leistungsfähige Sorten der Kulturpflanzen, aber auch verbesserte Anbaumethoden sowie eine schnell vorangetriebene Mechanisierung ihren Beitrag geleistet. Trotzdem wäre ohne die Erkenntnisse der Chemie seit Mitte des 19. Jahrhunderts ein solch steiler Aufstieg nicht möglich gewesen. Schadorganismen, Pilzkrankheiten und Unkräuter, aber auch schlechte Witterungsbedingungen verursachen weltweit Verluste von enormer wirtschaftlicher Bedeutung, welche bisweilen sogar existenzbedrohende Ausmaße annehmen können. Die Chemie hat der Landwirtschaft mit dem Mineraldünger und den Pflanzenschutzmitteln hierbei zwei herausragende Helfer mit auf den Weg gegeben.

Ziel dieses Kapitels ist es, einen Überblick über den Stand des modernen Pflanzenschutzes zu geben und die Bedeutung der Synthesechemie für die Agrarindustrie in der Vergangenheit sowie der heutigen Zeit aufzuzeigen.

\subsection{Die Geschichte des Pflanzenschutzes ${ }^{1,2}$}

Die ersten wirklich bedeutenden Ackerbaukulturen entwickelten sich vor rund 6000 Jahren in Ägypten und im Vorderen Orient. Aus Wildpflanzen entstanden durch Selektion des Menschen Kulturpflanzen und durch ausgedehnte Bewässerungswirschaft, wie sie z. B. in Mesopotamien zu finden war, konnten so mehr Menschen als je zuvor ernährt werden.

Aus Schriften der Sumerer sowie verschiedensten ägyptischen Darstellungen ist ersichtlich, dass die damaligen Kulturen mit den gleichen Problemen wie heutige Landwirte zu kämpfen hatten. Auch historische Quellen der Griechen und Römer überliefern Pflanzenschäden durch Insekten, Nagetiere und Pilzkrankheiten. Für den Schutz ihrer Vorräte hatten die Menschen damals schon viele Möglichkeiten. Den 
Pilzkrankheiten waren sie jedoch ziemlich hilflos ausgeliefert, da deren wahre Ursachen den früheren Kulturen nicht bekannt waren. Auch der Kampf gegen Fraßschäden durch Insekten stellte sich schwieriger dar als heute, da man nicht in der Lage war, diese abzutöten. Die Römer z. B. verwendeten deshalb Abschreckungsmittel (Reppelents), wie Asche, gestoßene Zypressenblätter oder verdünnten Urin, um die Schäden so gering wie möglich zu halten. Nach dem Zusammenbruch des Römischen Reiches bis in die frühe Neuzeit wurden keine verlässlichen Aufzeichnungen mehr geführt, so dass über die Landwirtschaft zu dieser Zeit nur wenig überliefert ist. Es ist jedoch nicht anzunehmen, dass es weniger Probleme als in den Jahrhunderten zuvor gegeben hat.

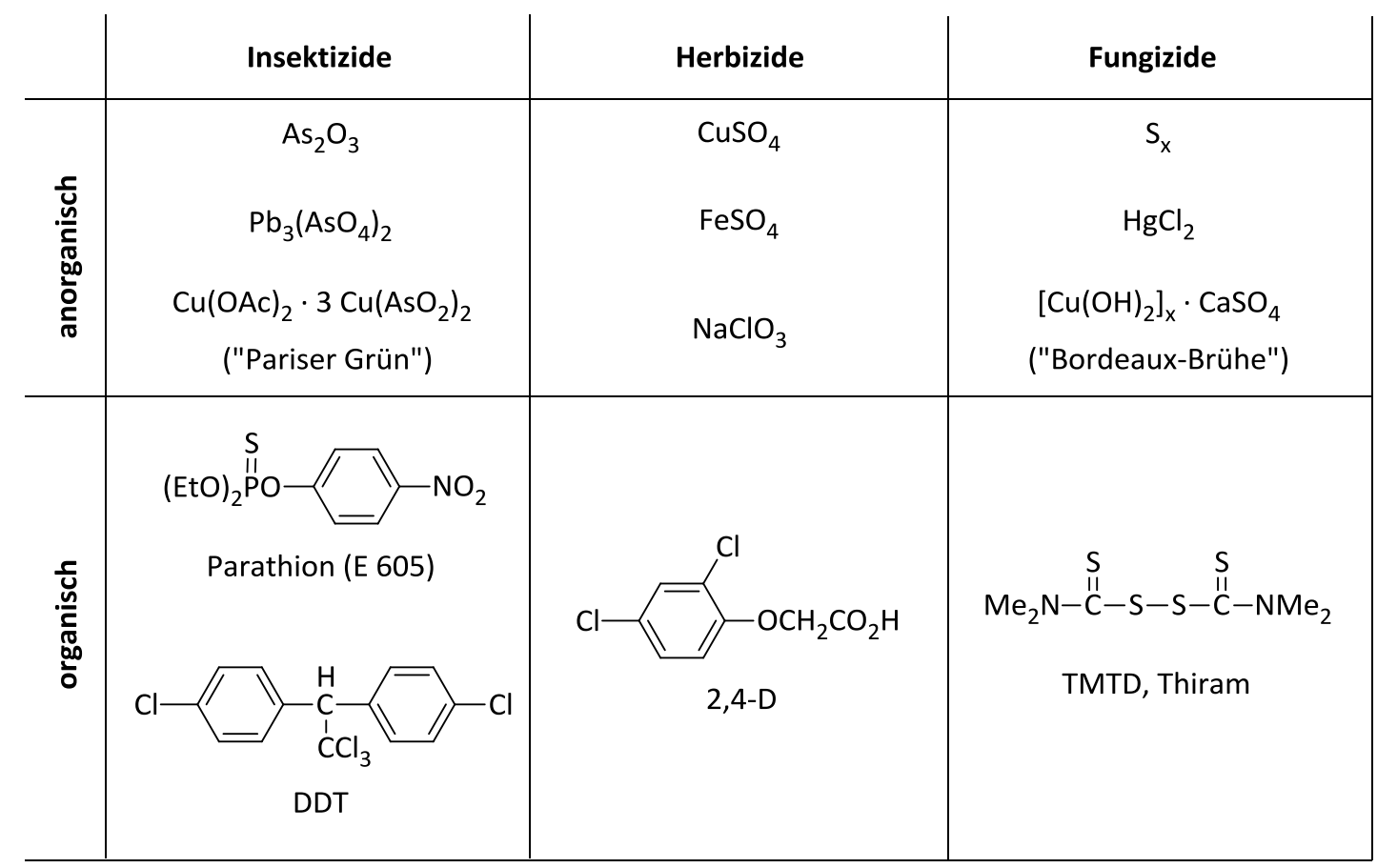

Abbildung 1: $\quad$ Beispiele für historische Pflanzenschutzmittel nach Stetter et al.

Eine neue Epoche des Pflanzenschutzes wurde ab der zweiten Hälfte des 18. und im Verlauf des 19. Jahrhunderts durch die Entdeckung wirksamer Naturstoffe zur Bekämpfung von Insekten eingeläutet. Hier ist vor allem das um das Jahr 1840 entdeckte Pyrethrum zu nennen, welches aus den getrockneten Blüten von Chrysanthemum cinerariifolium isoliert und als Insektenpulver verwendet wurde. Die ersten brauchbaren chemischen Mittel zum Schutz von Pflanzen waren meist anorganischer Natur und in der Regel recht unselektiv, so dass sie in großen Mengen aufgebracht werden mussten und zudem häufig auch für den Menschen toxisch waren. Sie waren 
meist Zufallsentdeckungen, wie die legendäre Bordeaux-Brühe, und stellten die ersten bescheidenen Waffen im Kampf gegen Seuchen im Kartoffel- und Weinbau des ausgehenden 19. Jahrhunderts dar. Ätzende Metallsalze, wie Kupfer- und Eisensulfat, aber auch verdünnte Säuren, wie Schwefel- und Salpetersäure, wurden zur Bekämpfung von Unkräutern verwendet (siehe Abbildung 1).

Eine wirkliche Revolution der agrochemischen Industrie begann erst in den 30er Jahren des 20. Jahrhunderts mit der Einführung einer planmäßigen Forschung, die das Ziel hatte, mindertoxische und selektive organisch-chemische Wirkstoffe zu entwickeln und im Pflanzenschutz zur Anwendung zu bringen. Die Erschließung leicht zugänglicher, breit anwendbarer, aber durchaus sehr effektiver Mittel stellte den ersten Durchbruch dar. Vor allem die Entwicklung von Organophosphor- (z. B. Parathion $[E \text { 605] }]^{3}$ ), Organochlor- (z. B. DDT $\left.{ }^{4}, 2,4-D^{5}\right)$ und Carbonsäurederivaten (z. B. Thiram, aber auch Carbamate) ${ }^{6}$ legten den Grundstein für eine prosperierende Pflanzenschutzmittelindustrie. Auch die Akzeptanz der Anwender war ausgesprochen hoch. Schließlich verminderten die neuen Pflanzenschutzmittel das betriebswirtschaftliche Risiko und den zu betreibenden Aufwand bei stark erhöhten Erträgen.

Bei den Konsumenten und in der breiten Öffentlichkeit war die Situation allerdings eine andere, führten die neuen synthetischen Wirkstoffe doch zu bisher nicht aufgetretenen Problemen. Einige Jahre nach ihrer Einführung stellte sich heraus, dass sich z. B. die Organochlorinsektizide (wie DDT oder Lindan) aufgrund ihrer hohen Persistenz in der Nahrungskette anreichern, indem sie sich im Fettgewebe von Tieren und Menschen einlagern. Diese heute als POPs (Persistent Organic Pollutants; langlebige organische Schadstoffe) bezeichneten Verbindungen führten zu ernsthaften Umweltschäden sowohl in Erd- als auch in Wasserökosystemen. ${ }^{7}$ Darüber hinaus stehen sie unter Verdacht, Schädigungen des Nervensystems, schnelleres Altern sowie Brustkrebserkrankungen zu verursachen. ${ }^{8}$ Der Höhepunkt der öffentlichen Diskussion über das Verhältnis von Chemie und Landwirtschaft war das Erscheinen von Rachel Carsons Buch „Silent Spring “9 übermäßigen Gebrauchs der damaligen Pflanzenschutzmittel auf das Ökosystem beschrieb und so die Bevölkerung auf dieses Thema aufmerksam machte. Schließlich 
wurde das Zeitalter der POPs mit deren Verbot durch das Inkrafttreten der Stockholmer Konvention im Jahre 2004 endgültig beendet. Es darf jedoch nicht vergessen werden, welche unschätzbaren Dienste Wirkstoffe wie DDT bei der Bekämpfung der Moskitos und somit der Malaria in den tropischen und subtropischen Gebieten der Erde geleistet haben. Berichten der Weltgesundheitsorganisation WHO zufolge konnten mit Hilfe von DDT viele Millionen Menschen vor dem Malariatod bewahrt werden.

\subsection{Anforderungen an neue Wirkstoffe $\mathrm{e}^{1,2}$}

Fast unbemerkt von der Öffentlichkeit hat in den vergangenen Jahren ein Prozess stattgefunden, in dessen Verlauf sich die Agrochemie zu einer High-Tech-Industrie entwickelt hat. Die heutige Forschung und Entwicklung im Pflanzenschutz unterscheidet sich in der Wissenschaftlichkeit und den hohen Qualitätsansprüchen kaum noch von der Arzneimittelforschung und -entwicklung. Frühere Pflanzenschutzmittelgesetze regelten lediglich den Schutz der Kulturpflanzen und forderten eine Anerkennung der Mittel. Heute hingegen besteht eine Zulassungspflicht und die Hersteller müssen neben der Wirkung auch die toxikologische Unbedenklichkeit für Anwender, Konsumenten und Umwelt gewährleisten. Die Zeiten, in denen die einzigen bekannten Fakten eines Pflanzenschutzmittels deren chemische Struktur und ihre beobachtete nützliche Wirkung waren, sind längst vorbei. Heute gehören sie ohne jeden Zweifel zu den am besten untersuchten Chemikalien, mit denen der Mensch und die Umwelt in Kontakt kommen. Dabei sind die Anforderungen an ein modernes Pflanzenschutzmittel stetig gestiegen und sehr vielfältig. Neben einer hohen spezifischen Wirkung auf den Schadorganismus sollte es günstige toxikologische Eigenschaften aufweisen, d. h. keine oder nur geringe Toxizität gegenüber Nutzorganismen sowie eine hervorragende Umweltverträglichkeit. Darüber hinaus sollte es eine gute Pflanzenverträglichkeit zeigen sowie eine für die benötigte Wirkung möglichst geringe Aufwandmenge besitzen. Dies ist z. B. durch eine systemische Wirkung zu erreichen. Um eine ständige Verfügbarkeit des Wirkstoffs zu gewährleisten muss die Gewinnung oder Herstellung unkompliziert und wirtschaftlich sein. Die wichtigste Voraussetzung 
ist jedoch ein verändertes Wirkungsspektrum mit möglichst neuem Wirkmechanismus, um die Gefahr von Kreuzresistenzen mit anderen Wirkstoffen zu vermeiden. So ist es nicht verwunderlich, dass die durchschnittliche Entwicklungszeit von der Synthese im Labor bis hin zur Zulassung als Handelsprodukt eine Zeitspanne von 8-12 Jahren umfasst.

Das zentrale Schlagwort der modernen Landwirtschaft heißt integrierter Pflanzenbau. Dies ist ein Gesamtkonzept, welches die ökologischen und wirtschaftlichen Aspekte des Pflanzenschutzes auf der Basis einer nachhaltigen Entwicklung verknüpfen soll. Das primäre Ziel ist eine Verminderung des Einsatzes chemischer Pflanzenschutzmittel auf das notwendige Minimum. Dies soll durch die Anwendung biologischer, biotechnischer sowie anbau- und kulturtechnischer Maßnahmen oder durch gezielte Züchtung geschehen. Die Umsetzung des integrierten Pflanzenbaus und die Harmonisierung des europäischen Pflanzenschutzrechts wird durch die Verordnung (EG) Nr. 1107/2009 geregelt, die zum 1. Januar 2014 für alle Mitgliedstaaten der Europäischen Union verpflichtend wird. ${ }^{10}$ Die Bundesregierung hat bereits darauf reagiert und im August 2011 den Gesetzentwurf zur Neuordnung des Pflanzenschutzrechts beschlossen. ${ }^{11}$

\subsection{Fungizide $e^{12}$}

Weltweit sind etwa 120000 Pilzarten bekannt, die auch etliche unserer Kulturpflanzen besiedeln. Dies macht sie zu einem ernsthaften Nahrungskonkurrenten für die Menschen. Darüber hinaus sind sie besonders in der Vergangenheit die Ursache für schwerste Erkrankungen, wie z. B. die „Mutterkornkrankheit“, gewesen, durch die viele Menschen den Tod gefunden haben. Schätzungen der FAO (Food and Agriculture Organization of the United Nations) zufolge werden Jahr für Jahr Millionen Tonnen an Nahrungsmitteln durch Pilzkrankheiten vernichtet. Aus diesem Grund ist eine stetige Bekämpfung pathogener Pilze an Kulturpflanzen notwendig. Laut Umweltbundesamt wurden in Deutschland im Jahr 2010 insgesamt Fungizide in einer Menge von 10431 t verkauft, womit sie knapp vor den Insektiziden auf Platz 2 der Pflanzenschutzmittel rangieren. ${ }^{13}$ 
Für einen fungiziden Wirkstoff gibt es verschiedene Wirkorte, da Pilze entweder als entwickelte Organismen im Inneren der Pflanze existieren oder in Form von Sporen auf der Pflanzenoberfläche zu finden sind. Daher lassen sich die Fungizide im Allgemeinen in zwei Klassen einteilen. Die protektiven Fungizide sind Kontaktfungizide, die auf der Pflanzenoberfläche angewendet werden, um eine Keimung und/oder eine Penetration des Keimschlauches zu verhindern. Die kurativen Fungizide sind vor allem systemische Wirkstoffe, die durch das Blatt oder die Wurzeln aufgenommen werden und dann über die Leitungsbahnen in der Pflanze verteilt werden. Dies ermöglicht auch die Bekämpfung von versteckt liegenden Krankheitsherden, die sich nach einer Infektion ausbilden. Die systemische Wirkung darf dabei allerdings nicht zu hoch werden, da der Wirkstoff sonst voll dem Metabolismus der Pflanze ausgesetzt ist. Dies kann einen schnelleren Abbau zu unwirksamen Stoffwechselprodukten zur Folge haben.

Moderne Wirkstoffe befinden sich zwischen den beiden beschriebenen Extremfällen. Sie sind weder reine Kontaktfungizide, noch wirken sie ausschließlich systemisch. Unter Verwendung spezieller Formulierungsmethoden können durch die Applikation regenfeste Wirkstoffdepots auf der Oberfläche der Blätter generiert werden, welche dann nach und nach das Fungizid in das Blatt entlassen und die Pflanze für mehrere Wochen befallsfrei halten. Dies hilft zudem Wirkstoff einzusparen, da sich die Häufigkeit der benötigten Anwendungen stark verringert. Gewöhnlich wird eine Mischung verschiedener Fungizide mit unterschiedlichen Eigenschaften ausgebracht, um die Pflanze sowohl protektiv als auch nach einer Infektion zu schützen.

Die Strukturmerkmale der Fungizide sind sehr vielfältig und auch deren Wirkmechanismen äußerst unterschiedlich. Die größte und am häufigsten verwendete Gruppe hat jedoch die mitochondriale Atmungskette als Ziel, in der die Wirkstoffe an verschiedenen Orten als Inhibitoren auftreten. Die prominentesten Vertreter dieser Klasse sind zweifelsohne die Strobilurine. Namensgeber dieser Klasse sind die Naturstoffe Strobilurin A (1) und B (2), welche aus dem Kiefernzapfenrübling Strobilurus tenacellus isoliert wurden. ${ }^{14}$ Nach ihrem strukturellen Vorbild und ihrer Wirkungsweise wurde eine Vielzahl von Wirkstoffen, wie z. B. Kresoxim-methyl (3) oder Pyra- 
clostrobin (4), entwickelt (Abbildung 2). Vor allem Letzterer ist in den letzten Jahren unter dem Namen F 500 zu einem weltweiten „Blockbuster" (Verkaufsschlager) aufgestiegen.

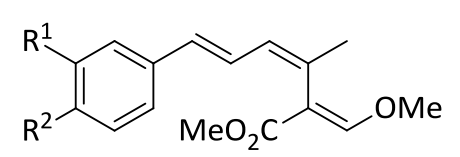

Strobilurin $A(1): \quad R^{1}=R^{2}=H$ Strobilurin $\mathrm{B}(2)$ : $\quad \mathrm{R}^{1}=\mathrm{OCH}_{3}, \mathrm{R}^{2}=\mathrm{Cl}$<smiles>CC(C)(C)OC(C)(C)C(C)(C)C</smiles><smiles>COC/C(=N/OC)c1ccccc1COc1ccccc1C</smiles>

Kresoxim-methyl (3)

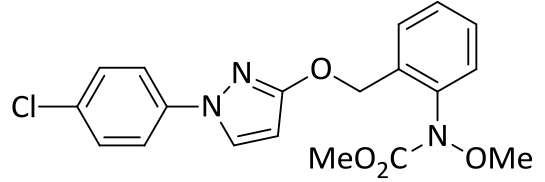

Pyraclostrobin (4)

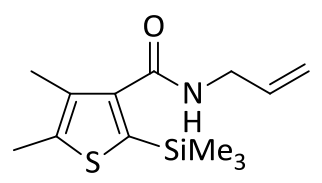

Silthiofam (7)

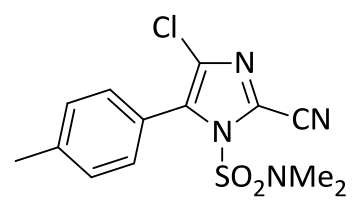

Cyazofamid (6)

Abbildung 2: Natürliche und neue Strobilurine sowie weitere Inhibitoren der Pilzatmung.

Wie vielfältig die Struktur selbst innerhalb der Gruppe der Atmungsketteninhibitoren ist, zeigen der Komplex-II-Hemmstoff Boscalid (5) aus der Klasse der Anilide, das Sulfonamid Cyazofamid (6) sowie das siliciumhaltige Saatbeizmittel Silthiofam (7).

Eine andere große Gruppe von Fungiziden greift in die Steroidbiosynthese der Pilze ein. Auch hier gibt es wieder eine Vielzahl an Orten in der Pflanze, an denen eine Hemmung vollzogen werden kann. Viele dieser Wirkstoffe sind innerhalb der Pflanze mobil, was ihnen die Eigenschaft verleiht, sowohl kurativ als auch protektiv wirksam zu sein. Die wichtigsten Vertreter sind hier die Demethylierungsinhibitoren (DMI), bei denen vor allem das Triazol Epoxiconazol (8) zu nennen ist (Abbildung 3). Die DMI werden häufig in Kombination mit anderen Fungiziden appliziert, was zu einer breiten Einsetzbarkeit im Getreide- und Gemüseanbau führt.

Aber auch die Inhibitoren des Zellwandaufbaus stellen äußerst potente Vertreter der Fungizide dar. Eine interessante Struktur weist hier der Wirkstoff Benthiavalicarb (10) auf, der mit L-Valin eine natürliche und mit D-Alanin eine unnatürliche Aminosäure enthält.

Bei den Melaninbiosynthese-Hemmern handelt es sich korrekterweise nicht um Fungizide, da der Pilz bei deren Einsatz nicht abgetötet wird. Es sind lediglich fungistatische Verbindungen, die den Pilz daran hindern, in die Epidermis der Pflanze 
einzudringen und somit in die Pathogenese einzugreifen. Ein Beispiel hierfür ist das optisch aktive Carpropamid (9).<smiles>Fc1ccc(C2(Cn3cncn3)OC2c2ccccc2Cl)cc1</smiles>

Epoxiconazol (8)<smiles>CCC1(C(=O)N[C@@H](C)c2ccc(Cl)cc2)C(C)C1(Cl)Cl</smiles>

Carpropamid (9)<smiles>CC(C)OC(=O)N[C@H](C(=O)N[C@@H](C)c1nc2ccc(F)cc2s1)C(C)C</smiles>

Abbildung 3: Beispiele für Inhibitoren der Steroid- und Melaninbiosynthese sowie des Zellwandaufbaus.

\subsection{Herbizide ${ }^{15}$}

Pflanzenarten, die mit den angebauten Kulturpflanzen um Nährstoffe, Sonnenlicht, Wasser und Raum konkurrieren, bezeichnet man als unerwünschte Begleitflora oder im allgemeinen Sprachgebrauch als Unkräuter. Aber auch auf Flughäfen, Bahngleisen und Autobahnen können Unkräuter zu erheblichen Schäden führen.

Die meisten Unkräuter werden vom Menschen selbst durch den Anbau von Kulturpflanzen verbreitet. Die Unkrautarten gedeihen unter ähnlichen Bedingungen und sind oft sogar mit den Kulturpflanzen verwandt. Dies macht es umso schwieriger die ungewollten Pflanzenarten selektiv zu bekämpfen. Neben einer chemischen Behandlung mit Herbiziden ist es ebenfalls möglich, den Unkrautbesatz durch bestimmte Fruchtfolgen einzuschränken. Darüber hinaus besteht die Möglichkeit, die Unkräuter manuell oder mechanisch zu entfernen. Dies ist jedoch meist nicht sehr effektiv und die Behandlungen müssen mehrmals wiederholt werden. In Ländern mit hohen Lohn- sowie Energiekosten sind diese beiden Methoden zudem unwirtschaftlich geworden und wurden deshalb durch den Einsatz von Herbiziden abgelöst. Im Jahr 2010 wurden deutschlandweit 16675 t an Herbiziden verkauft. ${ }^{13}$ Dies entspricht in etwa $40 \%$ der für den Schutz von Kulturpflanzen in Deutschland verwendeten 
Pflanzenschutzmittel. Damit stellen sie mit Abstand die wirtschaftlich wichtigsten Pflanzenschutzmittel dar.

Die Ausbringung landwirtschaftlich genutzter Herbizide erfolgt während verschiedener Anbauphasen. Daher können sie zunächst grob nach ihrer Einsatzzeit eingeteilt werden. Manche Produkte hemmen sowohl die Kulturpflanze als auch die Unkräuter im Wachstum. Diese nicht-selektiven Wirkstoffe werden entweder bereits vor bzw. während der Aussaat oder nach der Ernte verwendet. Andere Produkte werden ausgebracht, bevor der Keimling der Pflanze die Erdoberfläche durchbricht. Diese bezeichnet man als Vorauflauf-Herbizide. Der Einsatz sogenannter NachauflaufHerbizide erfolgt, wenn die Kulturpflanze bereits die Keim- oder Laubblätter ausgebildet hat.

Auch bei den Wirkstoffen der Herbizide gibt es eine Vielzahl von verschiedenen Wirkmechanismen. Es gibt in etwa 30 experimentell bestätigte Targets (Angriffsorte), die sich zumeist in den Chloroplasten der Pflanzenzellen befinden. Von diesen sind etwa 19 wirtschaftlich interessant. Zwei äußerst beliebte Angriffsziele stellen dabei die beiden Photosysteme dar.

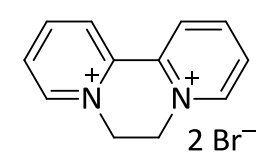

Diquat (11)

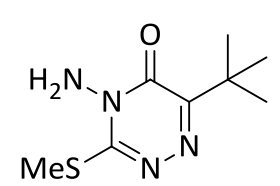

Metribuzin (13)

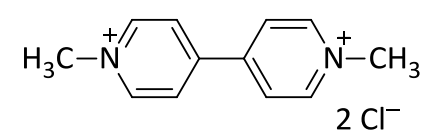

Paraquat (12)<smiles>CC(C)N1C(=O)c2ccccc2NS1(=O)=O</smiles>

Bentazon (14)<smiles>N#Cc1cc(Br)c(O)c(Br)c1</smiles>

Bromoxynil (15)

Abbildung 4: $\quad$ Beispiele für Inhibitoren der Photosysteme I und II.

Die prominentesten Vertreter der Photosystem-I-Herbizide sind die beiden nichtselektiv wirkenden Bipyridiniumsalze Diquat (11) und Paraquat (12) (Abbildung 4), deren Wirkung bereits in den 50er Jahren des letzten Jahrhunderts beschrieben wurde. Aufgrund toxikologischer Bedenken ist die Anwendung von Paraquat (12) jedoch in vielen Ländern nicht mehr erlaubt. Die Anzahl der Photosyntheseinhibitoren, welche die Funktion des Photosystems II beeinflussen, ist weitaus höher 
und stellt einen Großteil der weltweit verwendeten Herbizide dar. Hier spielen Wirkstoffe wie Metribuzin (13), Bentazon (14) oder Bromoxynil (15) eine wichtige Rolle (Abbildung 4). Vor allem letzteres ist ein sehr selektives Herbizid, welches neben seiner Eigenschaft als Photosyntheseinhibitor zusätzlich in die pflanzliche Atmung der Unkräuter eingreift. Dabei verhindert es den Aufbau des für die Synthese von ATP notwendigen Protonengradienten durch die Mitochondrien-Membran und stoppt so die Energiegewinnung des Unkrauts.

Neben den beiden Photosystemen sind aber auch die Biosynthesen verschiedener biochemisch relevanter Moleküle beliebte Targets. Hervorzuheben sind hier besonders die Synthese von Chlorophyll, der Carotinoide und der Aminosäuren.

Ein charakteristisches Strukturmerkmal vieler Carotinoid-Biosynthese-Inhibitoren ist die Trifluormethylphenyl-Teilstruktur, wie sie z. B. in Picolinafen (16) zu finden ist (Abbildung 5).
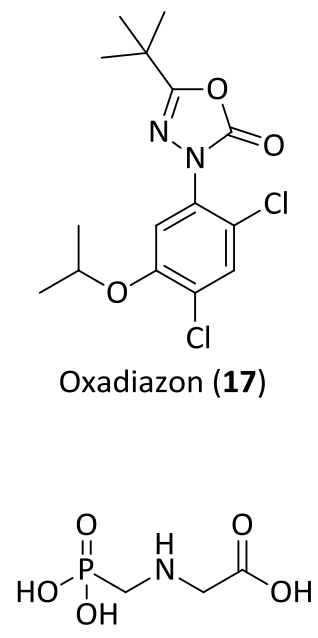

Glyphosat (18)
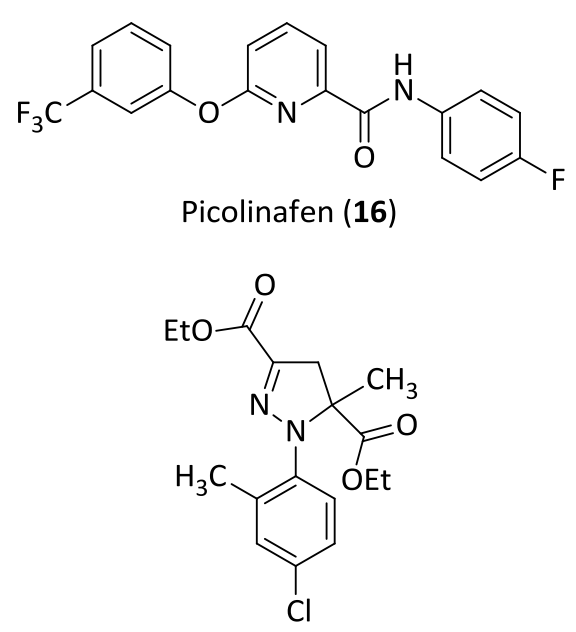

Mefenpyr-diethyl (19)

Abbildung 5: Beispiele für Biosynthese-Inhibitoren und Safener.

Ein gutes Beispiel für Wirkstoffe, die als Chlorophyll-Biosynthese-Hemmer fungieren, ist das Oxadiazon (17). Diese Wirkstoffgruppe basiert in auffällig vielen Fällen auf einer Heterocyclyl-Aryl-Grundstruktur, wie sie auch das Oxadiazol 17 trägt.

Der wohl bekannteste Vertreter der Aminosäure-Biosynthese-Inhibitoren und der Herbizide überhaupt ist das Glyphosat (18) (Abbildung 5). Dieses Phosphonsäuremethylglycerin-Derivat hemmt die Enolpyruvatshikimat-3-phosphat-Synthase (EPSPS), welche eines der zentralen Enzyme des Shikimisäurewegs ist. Dieser ist 
gemeinsamer Bestandteil der Biosynthese vieler aromatischer Pflanzeninhaltsstoffe, vor allem der aromatischen Aminosäuren Tryptophan, Phenylalanin und Tyrosin. Die günstigen herbizid-physiologischen Eigenschaften, wie die leichte Aufnahme über die Blätter und die schnelle Verteilung in der Pflanze, sowie die schnelle Abbaurate und die niedrigen Herstellungskosten haben Glyphosat (18) zum weltweit meistverkauften Pflanzenschutzmittel gemacht. Es wird in Nord- und Südamerika vor allem in Kombination mit gentechnisch veränderten Kulturpflanzen verwendet, denen künstlich ein Resistenzgen übertragen wurde, welches sie gegen Glyphosat unempfindlich macht. In den letzten Jahren sind jedoch verstärkt Resistenzen gegen den Wirkstoff aufgetreten, was den Weltmarkt für neu entwickelte Herbizide öffnet.

Eine bahnbrechende Entdeckung in der Herbizidsparte gelang den Forschern mit der Entwicklung von sogenannten Safenern (Antidots) ${ }^{16}$, wie das in Abbildung 5 gezeigte Mefenpyr-diethyl (19). Diese Verbindungen schützen selektiv die Kulturpflanze gegen die schädigende Wirkung des applizierten Herbizids, indem sie durch Aktivierung bestimmter Abbauenzyme metabolische Vorgänge in der Pflanze induzieren. Es besteht die Möglichkeit, Safener durch Beizung des Saatguts anzuwenden (SaatgutSafener), weit häufiger werden sie jedoch gemeinsam mit dem Herbizid als Mischung auf das Feld ausgebracht.

\subsection{Insektizide ${ }^{17}$}

Um die Qualität und den Ertrag der Erzeugnisse zu sichern, sind Insektizide zum Schutz der Kulturpflanzen und Nutztiere vor Schädlingsbefall nicht mehr aus der Landwirtschaft wegzudenken. Die wichtigsten Schädlinge lassen sich in die Gruppen (saugende und beißende) Insekten, Milben (Acari) und Zecken sowie Nematoden (Fadenwürmer) einteilen. Allerdings handelt es sich auch bei vielen Nützlingen um Tiere dieser Arten. Bienen und Hummeln sind z. B. wichtige Helfer bei der Bestäubung der Kulturpflanzen und bestimmte räuberische Milbenarten ernähren sich von Schädlingseiern, weshalb sie im Rahmen der biologischen Schädlingsbekämpfung und dem integrierten Pflanzenschutz zum Einsatz kommen. Bei den Insektiziden muss es sich also bestenfalls um Wirkstoffe handeln, die eine hohe Toxizität gegenüber den 
Schadorganismen zeigen und gleichzeitig Nützlinge, Anwender sowie die Umwelt schonen. Wie bei allen anderen Klassen der Pflanzenschutzmittel spielt auch bei den Insektiziden die systemische Wirkung eine wichtige Rolle, um die Aufwandmenge des Wirkstoffs zu verringern und einen umfassenden Schutz der Pflanze zu garantieren.

Eine beliebte Methode, die unglaubliche Vielzahl an Insektiziden in Gruppen einzuteilen, ist die Einordnung der Substanzen gemäß ihres Wirkmechanismus (mode of action). Nur ein kleiner Teil (ca. 5\%) der heutzutage auf dem Markt befindlichen Insektizide greift in die Entwicklung der Schädlinge ein. Dies geschieht auf der einen Seite durch Hormonmimetika oder Inhibitoren der Chitinbiosynthese, auf der anderen Seite durch Wirkstoffe, welche die mitochondriale Atmungskette bzw. die oxidative Phosphorylierung hemmen. Der weitaus größere Teil (etwa 80\%) der Insektizide wirkt jedoch als Neurotoxine, bei denen besonders die Neurotransmitter, wie Acetylcholin, und deren Rezeptoren beliebte Angriffsziele darstellen. Die Wirkmechanismen der übrigen etwa $15 \%$ der Insektizide sind bis heute nicht aufgeklärt.

Chitin bildet zusammen mit verschiedenen Proteinen das äußere Skelett der meisten Schädlinge. Benzoylharnstoffe wie das Diflubenzuron (20) (Abbildung 6) sind dafür bekannt, dass sie die Biosynthese von Chitin inhibieren, so dass die Schädlinge während ihrer Entwicklung nicht mehr dazu fähig sind, eine neue Cuticula (Außenhaut) auszubilden. Das Absterben der Tiere erfolgt jedoch erst mit einer gewissen Verzögerung im Zuge der nächsten regulären Häutung.

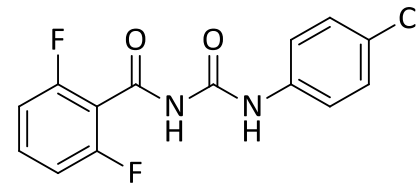

Diflubenzuron (20)<smiles>CCOC(=O)NCCOc1ccc(Oc2ccccc2)cc1</smiles>

Fenoxycarb (21)

Abbildung 6: $\quad$ Chitinbiosynthese-Inhibitor 20 und Juvenilhormon-Mimetikum 21.

Auf eine andere Art und Weise greift der in Abbildung 6 dargestellte Wirkstoff Fenoxycarb (21) in die Entwicklung des Schädlings ein. Indem es als Mimetikum für die Juvenilhormone wirkt, täuscht es die dauernde Anwesenheit dieser Hormone vor. Dies führt dazu, dass die Schädlinge in ihrer Weiterentwicklung gestört werden, da 
z. B. der Übergang vom Larvenstadium zum fortpflanzungsfähigen adulten Insekt ausbleibt.

Es ist sinnvoll, die große Gruppe der Neurotoxine nochmals nach ihrem genauen Angriffsort im Nervensystem in verschiedene Klassen einzuteilen. Die Reizleitung im Nervensystem erfolgt innerhalb einer Nervenzelle durch Membranpotenzialänderungen. Bei der Kommunikation zwischen zwei Nervenzellen, also bei der Weiterleitung eines Reizes über interzelluläre Kontaktstellen (Synapsen), spielen die sogenannten Neurotransmitter eine wichtige Rolle.<smiles>CCOP(=S)(OCC)Oc1ccc([N+](=O)[O-])cc1</smiles>

Parathion (22)<smiles>CN1CCC[C@H]1c1cccnc1</smiles>

(S)-Nicotin (24)<smiles>CNC(=O)Oc1cccc2c1OC(C)(C)C2</smiles>

Carbofuran (23)<smiles>O=[N+]([O-])/N=C1\NCCN1Cc1ccc(Cl)nc1</smiles>

Imidacloprid (25)

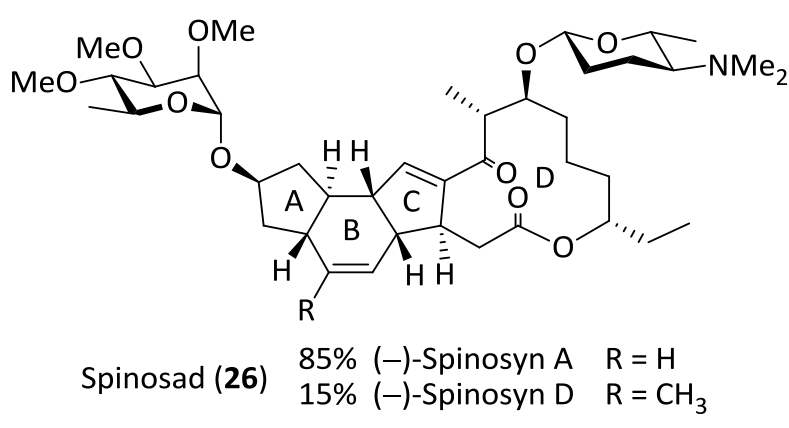

$\begin{array}{lll}\text { Spinosad (26) } & 85 \%(-) \text {-Spinosyn A } & \mathrm{R}=\mathrm{H} \\ 15 \%(-) \text {-Spinosyn D } & \mathrm{R}=\mathrm{CH}_{3}\end{array}$

Abbildung 7: Beispiele für Acetylcholin-Esterase-Inhibitoren sowie Acetylcholin-Rezeptor-Agonisten.

Eine Vielzahl an Wirkstoffen verhindert den Abbau des Neurotransmitters Acetylcholin durch Inhibierung der Acetylcholin-Esterase, so dass die reguläre Abschaltung des Reizes nicht erfolgen kann. Dies führt zunächst zur Lähmung des Tieres und anschließend zum Tod. Dominiert wird diese Wirkstoffklasse von den Carbamaten und den Organophosphaten. Vor allem aufgrund ihrer strukturellen Einfachheit und ihrer kostengünstigen Herstellung sind aus ihnen eine Fülle an Insektiziden wie Parathion (22) oder Carbofuran (23) hervorgegangen (Abbildung 7). Da die Reizweiterleitung von Säugetieren auf die gleiche Weise funktioniert, sind einige ihrer Vertreter jedoch nicht nur toxisch für Schädlinge, sondern auch für Säugetiere. Deshalb haben in den letzten Jahren viele von ihnen (auch die beiden oben genannten) ihre Zulassung in der Europäischen Union verloren.

Eine andere wichtige Gruppe von Insektiziden tritt als Agonisten des AcetylcholinRezeptors auf. Indem sie die Andockstellen für Acetylcholin blockieren bzw. selbst aktivieren, unterbrechen sie die kontrollierte Reizleitung im Schadorganismus. Die 
meisten Vertreter stammen aus der Stoffklasse der Neonicotinoide. Der Name dieser Stoffklasse leitet sich von dem Naturstoff Nicotin (24) (Abbildung 7) ab, bei dem erstmals diese Art von Wirkmechanismus beschrieben wurde. Sie sind hochwirksame Insektizide und zeichnen sich zudem durch eine hohe systemische Wirkung sowie eine sehr geringe Säugertoxizität aus. Der bekannteste Wirkstoff dieser Klasse ist das 1991 eingeführte Imidacloprid (25), welches eines der meistverkauften Pflanzenschutzmittel weltweit ist und nach dessen Vorbild eine Vielzahl neuer Wirkstoffe entwickelt wurde. Auch der sehr interessante Wirkstoff Spinosad (26) zählt zu der Klasse der Acetylcholin-Rezeptor-Agonisten, jedoch ist der genaue Angriffsort bis heute nicht aufgeklärt. Es ist nur bekannt, dass es sich um einen anderen RezeptorSubtyp als bei den Neonicotinoiden handeln muss (Abbildung 7).

Darüber hinaus gibt es noch die lonenkanal-Modulatoren. Wie bereits erwähnt, sind elektrische Signale in Form von Membranpotenzialänderungen für die intrazelluläre Reizleitung verantwortlich. Diese werden durch das Öffnen bzw. Schließen von Ionenkanälen, wie den spannungsabhängigen Natriumkanälen oder den Chloridkanälen gesteuert. Beide Kanäle sind hervorragende Targets und ihre Modulation führt zu einer Hyper-Erregung, die dann in der Lähmung des Schädlings endet. Die bekanntesten Vertreter der Natriumkanal-Modulatoren sind zweifelsohne die Pyrethroide; aber auch die Semicarbazone, zu denen das Metaflumizon (29) gehört, sind eine interessante Wirkstoffgruppe. Die Pyrethroide sind eine riesige Stoffklasse von hochwirksamen und selektiven synthetischen Insektiziden, deren Struktur und Wirkmechanismus sich von dem Naturstoff Pyrethrum ableitet. Abbildung 8 zeigt den Wirkstoff Alpha-Cypermethrin (28), der als 1:1-Gemisch der beiden Enantiomere zur Bekämpfung von Schadorganismen eingesetzt wird. Auch der Chloridkanal-Modulator Fipronil (27) wird als racemisches Gemisch appliziert und findet neben seiner Verwendung im Ackerbau auch in der Veterinärmedizin und im Haushaltsbereich Einsatz. 
<smiles>N#CC1NN(c2c(Cl)cc(C(F)(F)F)cc2Cl)C(N)C1C(=O)C(F)(F)F</smiles>

Fipronil (27)<smiles>N#Cc1ccc(C/C(=N\NC(=O)Nc2ccc(OC(F)(F)F)cc2)c2ccc(C(F)(F)F)cc2)cc1</smiles>

Metaflumizon (29)<smiles>C[C@H](OC(=O)[C@@H]1[C@H](C=C(Cl)Cl)C1(C)C)c1cccc(Oc2ccccc2)c1</smiles><smiles>[CH][C](OC(=O)[C@H]1[C@H](C=C(Cl)Cl)C1(C)C)c1cccc(Oc2ccccc2)c1</smiles>

Alpha-Cypermethrin (28)

(1:1-Gemisch der Enantiomere)

Abbildung 8: $\quad$ Beispiele für lonenkanal-Modulatoren.

Neben den üblichen Ausbringungsarten der Saatbeizung und Spritzapplikation gibt es für die Insektizide noch eine weitere Möglichkeit, den Schädling mit dem Wirkstoff in Kontakt zu bringen. Durch Pheromone werden z. B. die männlichen Artgenossen einer Schädlingspopulation zunächst zu einer Falle gelockt, in der sie mit dem Wirkstoff in Berührung kommen und sterben. Dieses Verfahren hat den großen Vorteil, dass die Kulturpflanze an sich nicht mit dem Insektizid in Kontakt kommt. Jedoch sind die Einsatzmöglichkeiten trotz großer Anstrengungen von Seiten der agrochemischen Industrie bis heute beschränkt. 


\section{Die Naturstoffklasse der Spinosyne ${ }^{18,19}$}

\subsection{Entdeckung, Identifizierung und Strukturmerkmale}

Um neue chemische Verbindungen mit möglichst ausgeprägter biologischer Aktivität zu finden, ist die Isolierung von Mikroorganismen aus Bodenproben und das Screening ihrer Fermentationsbrühen ein langbewährtes Mittel. Dabei besteht allerdings die Gefahr, dass man eine äußerst wertvolle Verbindung, die unter all den Produkten der Fermentationsbrühe in einer kaum zu detektierenden Menge vorliegt, übersieht.

Im Rahmen eines solchen Screening-Programms wurde auch die Naturstoffklasse der Spinosyne entdeckt. Aus einer Bodenprobe vom Gelände einer stillgelegten Rumdestillerie auf den Jungferninseln konnte im Jahre 1982 der Actinomycet Saccharopolyspora spinosa isoliert werden. ${ }^{20}$ Die beiden Hauptmetabolite dieses aeroben, grampositiven und Mycel-bildenden Bakteriums sind die in Abbildung 9 dargestellten Verbindungen $\mathbf{3 0}$ und 31. In Anlehnung an den Artennamen der Spezies wird diese Klasse von Verbindungen als Spinosyne bezeichnet. Der aktivste Vertreter ist das Spinosyn A (30), welches zusammen mit Spinosyn D (31) von dem Organismus ungefähr im Verhältnis 85:15 produziert wird.

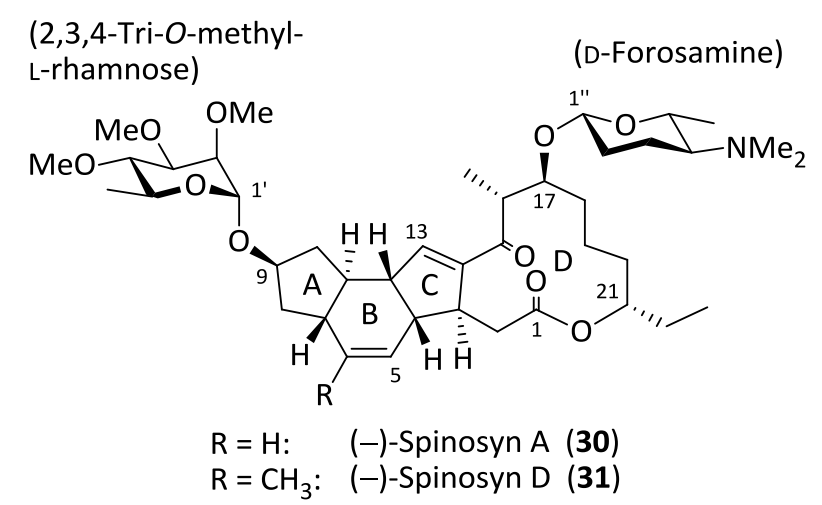

Abbildung 9: $\quad$ Hauptmetabolite von Saccharopolyspora spinosa: Spinosyn A (30) und D (31).

Strukturell gesehen sind die Spinosyne Makrolide, deren Grundgerüst von einem tetracyclischen Ringsystem gebildet wird. Dieses Ringsystem, bestehend aus einem cis-anti-trans-5,6,5-Tricyclus, anelliert an ein 12-gliedriges Makrolacton, ist in der Natur einzigartig. Lediglich die beiden Antibiotika Ikarugamycin ${ }^{21}$ und Capsimycin ${ }^{22}$ 
zeigen ein ähnliches Grundgerüst, besitzen jedoch ein ganz anderes Substitutionsmuster und sind zudem an ein Makrolactam anelliert. Das tetracyclische Grundgerüst enthält neun stereogene Zentren. Zwei dieser stereogenen Zentren tragen Hydroxyfunktionen, an welche die 2,3,4-Tri-O-methyl-L-rhamnose (C-9) durch eine $\alpha$-Verknüpfung und der Aminozucker D-Forosamin (C-17) durch eine $\beta$-Verknüpfung gebunden sind. Mittlerweile wurden weit über 20 verschiedene Spinosyne isoliert und charakterisiert. Sie unterscheiden sich hauptsächlich in dem Methylierungsmuster der Amino- und Hydroxygruppen der Zuckereinheiten und in geringem Maße auch im Alkylierungsmuster des Grundgerüsts (C-6 und C-21).

Darüber hinaus wurde vor wenigen Jahren eine neue Saccharopolyspora-Spezies mit dem Namen Saccharopolyspora pogona entdeckt. Aus dieser konnten zusätzlich mehr als 30 weitere Spinosyne isoliert und charakterisiert werden. Viele dieser auch als Pogonine bezeichneten Verbindungen tragen an C-21 anstelle des Ethyl-Substituenten eine 2-Butenyl-Einheit, was ihnen zusätzlich den Namen Butenyl-Spinosyne einbrachte. $^{23}$

\subsection{Eigenschaften und Wirkungsweise}

Die erste Zulassung einer auf Spinosynen basierenden Wirkstoff-Zubereitung wurde 1997 in den USA erteilt. Dabei handelte es sich um den Wirkstoff Spinosad, dessen Name eine phonetische Zusammensetzung des Begriffs Spinosyn mit den beiden Faktorennamen A und D darstellt. Spinosad wird durch Fermentation gewonnen und enthält die Spinosyne A (30) und D (31) in einem Verhältnis von etwa 85:15, wie es auf natürliche Weise von Saccharopolyspora spinosa produziert wird. Bis heute wurden etliche Produkte mit Spinosad als Wirkstoff für verschiedenste Anwendungen in der Landwirtschaft entwickelt. Im Handel erhältlich sind sie weltweit unter Markennamen wie SpinTor ${ }^{\circledR}$, Entrust $^{\circledR}$, Tracer $^{\circledR}$, Conserve $^{\circledR}$ sowie Success $^{\circledR}$ und spielen auch im Rahmen des integrierten Pflanzenschutzes eine gewichtige Rolle. Aufgrund seines ausgezeichneten ökologischen Profils und der Tatsache, dass es sich um einen Naturstoff handelt, ist Spinosad sogar für die Verwendung im ökologischen Landbau zugelassen. Die Naturstoffklasse der Spinosyne zeichnet sich neben ihrer 
insektiziden Wirkung gegen Schädlinge, wie Lepidopteren (Schmetterlinge), Dipteren (Zweiflügler), Hymenopteren (Hautflügler), Thysanopteren (Fransenflügler) sowie Coleopteren (Käfer), auch durch ihre antiparasitäre Wirkung aus. Daher gibt es mittlerweile auch eine Reihe an Produkten, die in der Veterinärmedizin verwendet werden, um Nutz- und Haustiere vor dem Befall von blutsaugenden Parasiten zu schützen. Ganz aktuell aus dem Jahr 2011 ist die Zulassung des Spinosad-enthaltenden Produktes Natroba ${ }^{\mathrm{TM}}$ in den USA. Dies ist ein Mittel aus der Humanmedizin, welches zur äußerlichen Behandlung von Kopfläusen verwendet wird.

Resistenzen spielen im Bereich der Schädlingsbekämpfung eine immer größer werdende Rolle. Ein verantwortungsbewusstes Resistenzmanagement zielt darauf ab, neue Wirkstoffe zu entdecken, die einen möglichst neuartigen Wirkmechanismus (mode of action) besitzen, um die Gefahr von Kreuzresistenzen zu minimieren. Der mode of action der Spinosyne konnte bis zum heutigen Tag nicht vollständig aufgeklärt werden. Tabelle 1 gibt eine Übersicht über die typischen Angriffsorte der handelsüblichen, als Neurotoxine wirkenden Insektizide.

Wirkort

$\mathrm{Na}^{+}-$Kanal

Acetylcholin-Esterase

nACh-Rezeptor

GABA-Rezeptor

\section{Wirkstoffbeispiel}

Pyrethroide, DDT

Organophosphate, Carbamate

Neonicotinoide (z. B. Imidacloprid)

Avermectin, Fipronil

Tabelle 1: $\quad$ Wirkort und zugehöriger Wirkstoff bei verschiedenen Insektiziden bzw. Insektizidklassen.

Erste Studien führten zu der Vermutung, dass es sich bei dem Angriffsort der Spinosyne um einen noch nicht bekannten Subtypen des nicotinergen Acetylcholin(nACh)-Rezeptors handeln könnte. ${ }^{24}$ Außerdem wäre ein antagonistischer Effekt auf den $\gamma$-Aminobuttersäure (GABA)-Rezeptor nicht auszuschließen. ${ }^{25}$ Neueste Untersuchungen durch Tritium- $\left[{ }^{3} \mathrm{H}\right]$-Austauschexperimente führten auf jeden Fall zu dem Ergebnis, dass die Spinosyne keine der bisher bei anderen Insektiziden bekannten Bindungsstellen adressieren, und somit einen völlig neuartigen Wirkmechanismus 
besitzen müssen. ${ }^{26}$ Es konnte lediglich ein geringes Maß an Interaktion mit den spannungsabhängigen Calciumkanälen nachgewiesen werden. Auch wenn der genaue Wirkmechanismus des Spinosads nicht abschließend geklärt ist, sind die Auswirkungen auf den Schädling nach Kontamination mit dem Wirkstoff deutlich zu sehen. Es kommt zum Tremor, unkontrollierten Bewegungen der Mundwerkzeuge sowie hektischer Atmung bis hin zur vollständigen irreversiblen Lähmung des Schädlings. Dabei kann die Aufnahme des Wirkstoffs sowohl über Kontakt, als auch durch Fraßaktivitäten erfolgen. Die Wirkung setzt meist einige Minuten bis Stunden nach der Kontamination ein, wobei sie durch orale Aufnahme 5- bis 10-mal höher einzustufen ist als jene durch Kontakt. Durch Studien an der amerikanischen Tabakeule (Heliothis virescens) konnte zudem gezeigt werden, dass es sich bei Spinosad um einen langsam in den Schädling penetrierenden Wirkstoff handelt, der im Organismus selbst wenig bis gar nicht metabolisiert wird. Dies könnte dafür sprechen, dass die biologische Aktivität nicht auf einzelne Strukturmerkmale, sondern auf das Molekül als Ganzes zurückzuführen ist. ${ }^{27}$

Spinosyne zeigen eine außerordentlich hohe Selektivität gegenüber dem Zielorganismus, während sie nur eine geringe bis keine toxische Wirkung für Säugetiere, Vögel und Wasserlebewesen aufweisen (Tabelle 2). ${ }^{28,29}$

\begin{tabular}{|c|c|c|c|}
\hline Spezies & Test & Ergebnis & Einstufung \\
\hline $\begin{array}{l}\text { Ratte männl. } \\
\text { Ratte weibl. }\end{array}$ & Akute orale $\mathrm{LD}_{50}$ & $\begin{array}{l}>3738 \text { mg/kg Körpergewicht } \\
>5000 \text { mg/kg Körpergewicht }\end{array}$ & Nicht toxisch \\
\hline Kaninchen & Akute dermale $L_{50}$ & >5000 mg/kg Körpergewicht & Nicht toxisch \\
\hline Forelle & $96 \mathrm{~h}$ akute $\mathrm{LC}_{50}$ & $30 \mathrm{mg} / \mathrm{L}$ Wasser & Nicht toxisch \\
\hline Stockente & Akute orale $\mathrm{LD}_{50}$ & >2000 mg/kg Körpergewicht & Nicht toxisch \\
\hline Tabelle 2: & Toxische Wirkung von & pinosad auf ausgewählte Säugetiere, & Fische und Vögel. \\
\hline
\end{tabular}


auch für eine Vielzahl von Raubmilben sowie räuberischen Insekten nicht schädigend ist. Daher kann der Wirkstoff bedenkenlos im integrierten Pflanzenschutz eingesetzt werden. Eine wichtige Rolle unter den Nutzinsekten kommt den Bienen und Hummeln zu, da sie einen großen Anteil an der Bestäubung der Kulturpflanzen haben. Daher werden alle Insektizide in vier Kategorien nach ihrer Bienentoxizität eingeteilt: B4 (nicht bienengefährlich), B3 (nicht bienengefährlich, da durch Zulassung nur festgelegte Anwendungen des Mittels erlaubt sind), B2 (bienengefährlich, ausgenommen bei Anwendung nach dem täglichen Bienenflug bis 23.00 Uhr) und B1 (bienengefährlich). ${ }^{30}$ Spinosad ist in der Kategorie B1 zugelassen und unter Laborbedingungen akut toxisch für Bienen. ${ }^{31}$ In Feldversuchen konnte jedoch gezeigt werden, dass bei sachgemäßer Anwendung nur eine geringe Gefährdung für Bienen besteht. Wurden die Bienen z. B. bereits auf der Pflanze angetrockneten Wirkstoffrückständen ausgesetzt, konnten keinerlei Vergiftungserscheinungen festgestellt werden. ${ }^{32}$

Die hochpotente Wirkung des Spinosads zeigt sich vor allem dadurch, dass die auszubringende Wirkstoffmenge zur Kontrolle verschiedener Schädlingspopulationen in den meisten Fällen unter 100 g pro Hektar Ackerfläche liegt (Tabelle 3). ${ }^{28}$

\begin{tabular}{ccc}
\hline Spezies & Wissenschaftlicher Name & Aufwandmenge (g/ha) \\
\hline Maiszünsler & Ostrinia nubilalis & $25-50$ \\
Baumwollkapselbohrer & Helicoverpa zea & $40-100$ \\
Baumwollkapselwurm & Helicoverpa armigera & $50-100$ \\
Kohlmotte & Plutella xylostella & $15-50$ \\
Kohlweißling & Pieris rapae & $50-100$ \\
Traubenwickler & Lobesia botrana & $25-50$ \\
Tomatenschwärmer & Manduca quinquemaculata & $40-100$ \\
\hline
\end{tabular}

Tabelle 3:

Aufwandmengen für Spinosad im Bereich Insektenbekämpfung.

Neben einer hohen Wirksamkeit und Selektivität ist die Umweltverträglichkeit ein ganz entscheidender Faktor für die Etablierung eines Insektizids auf dem Weltmarkt. Wichtige Aspekte sind hierbei vor allem der Einfluss des Wirkstoffs auf die Grundwasserqualität sowie dessen mögliche Abbauprozesse in den oberen Bodenschichten und auf der Pflanzenoberfläche. 
Die Spinosyne sind relativ unpolare Verbindungen $(\log P=4.0$, bei $\mathrm{pH} 7$ für Spinosyn A) und besitzen eine schlechte Wasserlöslichkeit (235 mg/L bei pH 7). Besonders die schlechte Wasserlöslichkeit verhindert, dass der Wirkstoff in tiefere Bodenschichten gespült wird, so dass es zu keiner Kontamination des Grundwassers kommen kann. Darüber hinaus unterliegt es im Boden schnellen, mikrobiell gesteuerten Degradierungsprozessen. Auf der Pflanzen- und Bodenoberfläche sowie in Gewässern stellt die Photolyse den primären Abbaumechanismus dar. ${ }^{33}$ Unter direkter Sonneneinstrahlung beträgt die Halbwertszeit im Freiland in etwa einen Tag.

\subsection{Stand der gegenwärtigen Forschung}

Spätestens seit Beginn des neuen Jahrtausends wird vermehrt über die Entwicklung von Resistenzen sowohl im Labor $^{34}$ als auch in Feldstudien ${ }^{35}$ berichtet. Um diese rechtzeitig beherrschen zu können sowie die zukünftige Deckung des Wirkstoffbedarfs zu garantieren, ist eine weiterhin intensive Forschung auf dem Gebiet der Spinosyne unabdingbar. Neben der Optimierung der Fermentationsprozesse steht dabei vor allem die Erschließung alternativer Zugangsquellen, wie z. B. die chemische Totalsynthese oder die gentechnische Veränderung des Bakterienstamms, zur Herstellung neuer Derivate und Analoga im Mittelpunkt. Damit dies gelingt, müssen Studien zum Verständnis des Zusammenhangs zwischen Molekülstruktur und Aktivität durchgeführt werden, um so sinnvolle Modifikationen am Wirkstoff durchführen zu können.

\subsubsection{Naturstoffderivatisierung - Struktur-Aktivitäts-Beziehungen und Synthese von Spinosoiden}

Besonders im direkten Umfeld der industriellen Forschung wurden große Anstrengungen unternommen, um durch gezielte Derivatisierung neue semisynthetische Spinosyne (Spinosoide) herzustellen (Abbildung 10). Im Blickpunkt der Forschung stand dabei vor allem die Aufklärung der Struktur-Aktivitäts-Beziehung (SAR = structure activity relationship). Dabei bediente man sich später auch einer Computer-gestützten Variante, der sogenannten artificial-neural-network (ANN)basierten quantitativen Struktur-Aktivitäts-Beziehung, mit deren Hilfe weitere 
sinnvolle Modifikationen vorausgesagt werden konnten. ${ }^{36}$ Bis heute wurden so insgesamt mehr als 1000 Spinosoide synthetisiert, charakterisiert und auf biologische Aktivität getestet.

Rhamnose: Abspaltung, Ersatz,

Forosamin: Abspaltung, Ersatz, Modifikation des Substitutionsmusters Modifikation des Substitutionsmusters

Einführung zusätzlicher Doppelbindungen im A-Ring

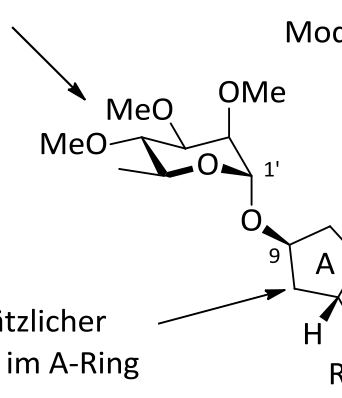

Modifikation des

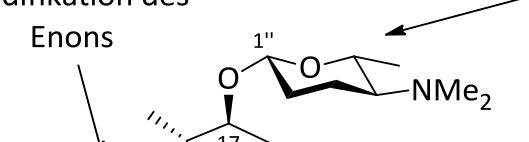<smiles>[2H]O[C@@H](C)[C@@H](C)/C(C)=C(\C)C(C)C</smiles>

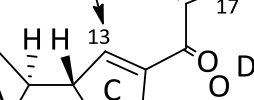

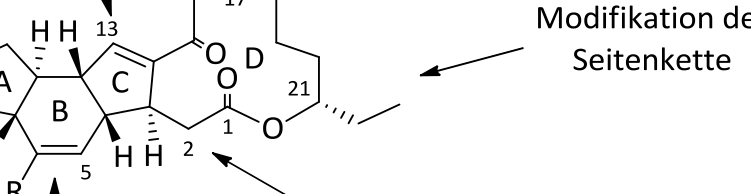
Allylsystem: Oxidation, C-2-Alkylierung Reduktion

Abbildung 10: Übersicht zu bereits durchgeführten Derivatisierungen im Rahmen von SAR-Studien.

Die ersten richtungsweisenden Arbeiten zur Aufklärung der Struktur-AktivitätsBeziehung wurden an den beiden Zuckereinheiten L-Rhamnose und D-Forosamin durchgeführt. Diese unterstrichen dabei die essentielle Rolle der beiden Zuckereinheiten, da deren Abspaltung zum völligen Verlust der insektiziden Wirkung führte. ${ }^{37}$

\begin{tabular}{|c|c|c|c|c|c|}
\hline Modifikation & Spinosyn & $\begin{array}{c}\mathrm{LC}_{50} \\
\text { [ppm] }\end{array}$ & Modifikation & Spinosyn & $\begin{array}{c}L_{50} \\
{[p p m]}\end{array}$ \\
\hline Spinosyn A (30) & $A$ & 0.3 & $13-\mathrm{NHOH}$ & $A$ & 5.6 \\
\hline Spinosyn D (31) & D & 0.8 & 5,6- $\beta$-Eроху & D & 10 \\
\hline Aglykon & $A$ & $>64$ & 5,6-Dihydro & $A$ & 0.5 \\
\hline $2^{\prime}, 3^{\prime}, 4^{\prime}-$ Tri-O-Et & $A$ & 0.02 & $5-\mathrm{OH}$ & $\mathrm{D}$ & $>64$ \\
\hline 4"-Keto & A & 3.4 & 2-Methyl & A & 4.5 \\
\hline $13,14-\beta$-Dihydro & A & 20 & 7,8-Dehydro (32) & $\mathrm{D}$ & 0.6 \\
\hline $13,14-\alpha$-Dihydro & $A$ & 4.7 & 7,11-Dehydro (33) & $\mathrm{D}$ & 0.2 \\
\hline 13,14-Epoxy & $A$ & 1.4 & Indenyl (34) & D & $>64$ \\
\hline
\end{tabular}

Tabelle 4: Insektizide Wirkung von ausgewählten Spinosynen und Spinosoiden gegenüber der amerikanischen Tabakeule (Heliothis virescens).

Positive Veränderungen konnten durch die Variation des Substitutionsmusters am Rhamnosylglykosid erzielt werden. Biologische Screenings dieser von Crouse et al. 
synthetisierten Spinosoide zeigten in manchen Fällen eine respektable Aktivitätssteigerung. ${ }^{38}$ Einige interessante Ergebnisse sind in Tabelle 4 dargestellt.

Erste Modifikationen am tetracyclischen Grundgerüst wurden am Enon- und 5,6-Allylsystem durchgeführt. Eine Epoxidierung der C=C-Doppelbindungen führte in beiden Fällen zu einer Aktivitätsverschlechterung. Auch die Einführung eines Alkylsubstituenten am C-2-Kohlenstoffatom hatte eine Verringerung der Wirkung zur Folge.

Gegenstand weiterer intensiver Untersuchungen war die Einführung zusätzlicher C=CDoppelbindungen zwischen und in dem A-B-Ringsystem des Spinosyns D (31). Dies hätte zusätzlich den Effekt, dass es zu einer Verringerung der Anzahl stereogener Zentren kommt und somit zu einer Verringerung der Komplexität. Abbildung 11 zeigt die synthetisierten Derivate.

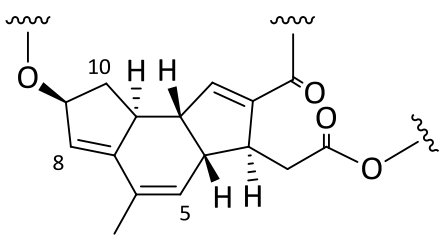

7,8-Dehydro-Spinosyn D (32)

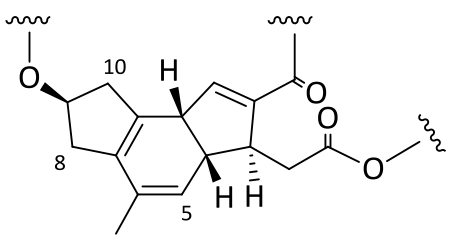

7,11-Dehydro-Spinosyn D (33)

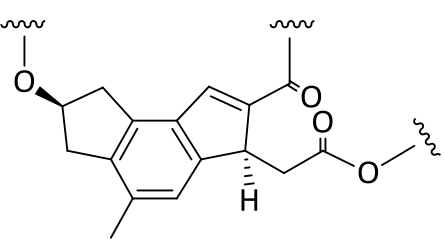

Indenyl (34)

Abbildung 11: $\quad$ Derivate von Spinosyn D mit zusätzlichen Doppelbindungen.

Trotz des Wegfalls der stereogenen Zentren durch die Einführung einer Doppelbindung in 7- und 8-Position bzw. in 7- und 11-Position wurden Aktivitäten erzielt, die mit $\mathrm{LC}_{50}=0.6 \mathrm{ppm}$ für Verbindung 32 und $\mathrm{LC}_{50}=0.2 \mathrm{ppm}$ für Verbindung $33 \mathrm{im}$ Bereich der wirksamsten Vertreter dieser Naturstoffklasse liegen (Tabelle 4). Das Indenylderivat $34\left(\mathrm{LC}_{50}>64 \mathrm{ppm}\right)$ zeigt hingegen einen vollständigen Verlust der insektiziden Wirkung.

Die Erkenntnisse dieser Studie sind sehr aufschlussreich, da sie beweisen, dass die stereogenen Zentren an C-7 und C-11 für die biologische Aktivität nicht benötigt werden, wohingegen die cis-Verknüpfung des B- und C-Rings unentbehrlich ist. Diese Interpretationen lassen die Annahme zu, dass eine Fortführung der Strukturvereinfachungen, z. B. durch Einführung eines aromatischen Cyclus anstelle des aliphatischen A-Rings, zu interessanten Bausteinen im Rahmen des Resistenzmanagements führen könnte. Untersuchungen zur Herstellung eines solchen strukturvereinfachten Derivats sind auch Gegenstand dieser Arbeit. 
Auf der Grundlage dieser Studien zur Struktur-Aktivitäts-Beziehung hat vor kurzem ein neuer semi-synthetischer Wirkstoff in verschiedenen Ländern unter den Namen Delegate $^{\circledR}$ und Radiant ${ }^{\circledR}$ die Zulassung als Pflanzenschutzmittel erhalten. Hierbei handelt es sich um den Wirkstoff Spinetoram, welcher ein Gemisch aus 3'-O-Ethyl5,6-dihydro-Spinosyn J (37) als Hauptkomponente und 3'-O-Ethyl-Spinosyn L (38) als Minderkomponente ist.

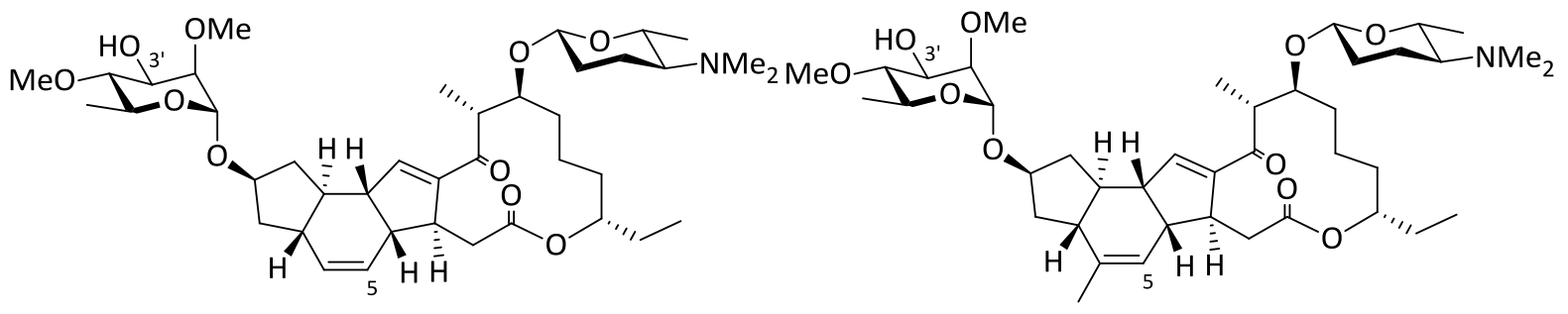

Spinosyn J (35)

Spinosyn L (36)

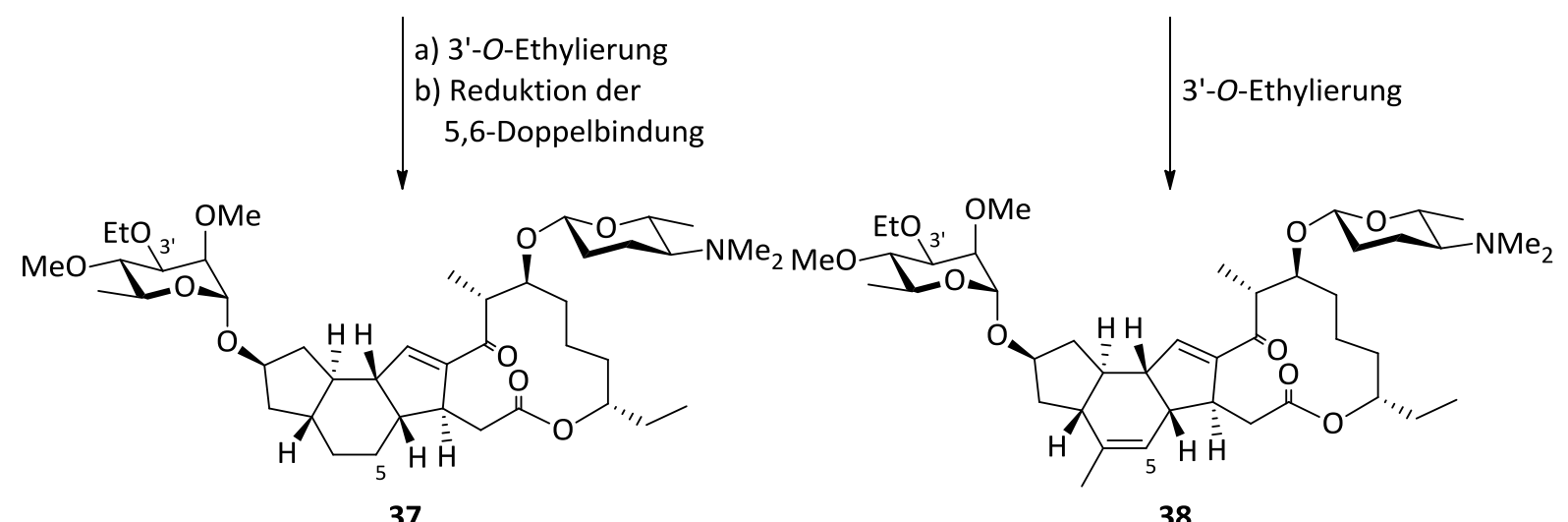

Abbildung 12: $\quad$ Semi-synthetischer Zugang zu Spinetoram.

Abbildung 12 beschreibt den Weg der beiden Spinosoide von dem natürlichen, durch Fermentation gewonnenen Gemisch von Spinosyn J (35) und L (36) bis zum fertigen Wirkstoff. Der erste Schritt ist eine Ethylierung der in beiden Spinosynanaloga frei vorliegenden Alkoholfunktion an C-3'. Die darauffolgende Hydrierung der 5,6-Doppelbindung ist bei dem Derivat des Spinosyns L durch die Anwesenheit der Methylgruppe in 6-Position gehindert und erfolgt daher nur bei dem von Spinosyn J abgeleiteten Derivat. Es konnte gezeigt werden, dass Spinetoram im Vergleich zu Spinosad eine höhere Toxizität gegenüber Schadorganismen sowie eine verbesserte Photostabilität aufweist. Außerdem wurde ein früheres Einsetzen der Wirkung und eine geringere Toxizität gegenüber Hummeln beobachtet. $^{39,40}$ 


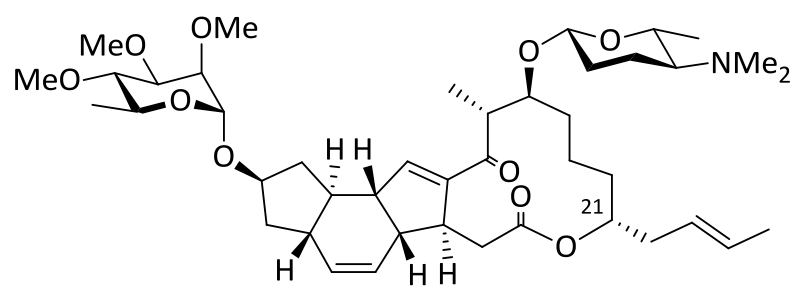

Spinosyn $\alpha 1$ (39)

Abbildung 13: Beispiel für die Struktur eines Butenyl-Spinosyns.

Zudem wurde kürzlich eine neue Unterklasse der Spinosyne entdeckt. Sie entstammt der Actinomyceten-Spezies Saccharopolyspora pogona, von der auch, wie bereits erwähnt, der Name Pogonine abgeleitet ist. Ein Großteil dieser Verbindungen besitzt an C-21 eine Butenyleinheit als Substituenten anstelle eines Ethylrestes, was dieser Gruppe zusätzlich den Namen Butenyl-Spinosyne eingebracht hat. ${ }^{23}$ Diese Verbindungen sind sehr gut dazu geeignet, die durch verschiedenste Manipulationen der Seitenkette an C-21 entstehenden Effekte auf die Aktivität der Spinosyne zu untersuchen (Abbildung 13).

Die ersten Testungen der insektiziden Wirkung der Butenyl-Spinosyne und deren semi-synthetischen Derivaten zeigten teilweise recht gute Aktivitäten, lagen aber im Bereich der bisher bekannten $\mathrm{LC}_{50}$-Werte. Allerdings konnten einige interessante neue Struktur-Aktivitäts-Beziehungen durch die Modifikation der Seitenkette an C-21 festgestellt werden. ${ }^{41}$

\subsubsection{Biosynthese ${ }^{42,43}$}

Die Aufklärung der Spinosyn-Biosynthese mit all ihren Schritten war in den letzten Jahren das Ziel zahlreicher Untersuchungen, welche in einer Vielzahl von Publikationen mündete. Frühere Arbeiten hatten bereits gezeigt, dass der Aufbau des Bausteins 40 über einen Typ-I-Polyketid-Synthase (PKS) Pfad erfolgt, wobei neun Acetat- und zwei Propioneinheiten verbraucht werden. In den neuen Studien konnten vier Gene (SpnA-E) im Spinosyn-A-Gencluster von Saccharopolyspora spinosa ausgemacht werden, die vermutlich die Enzyme für die Umsetzung zu dem Produkt 40 der Polyketid-Synthase (PKS) exprimieren (Abbildung 14). 


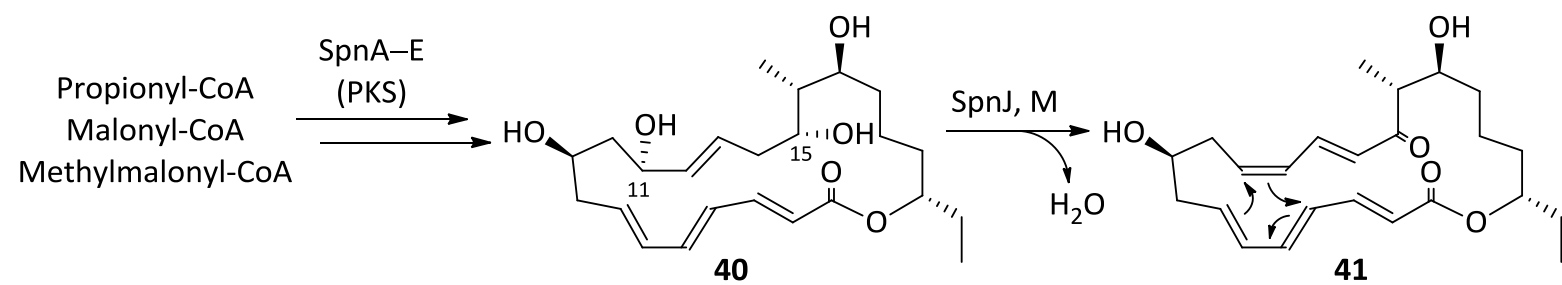

40

41

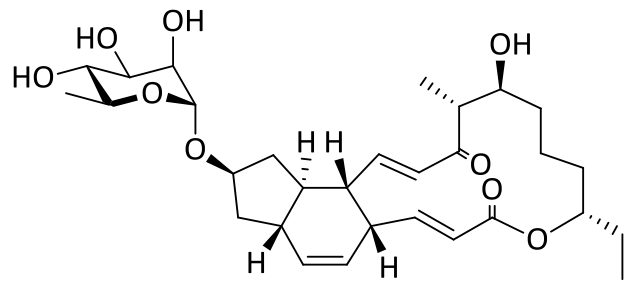

43

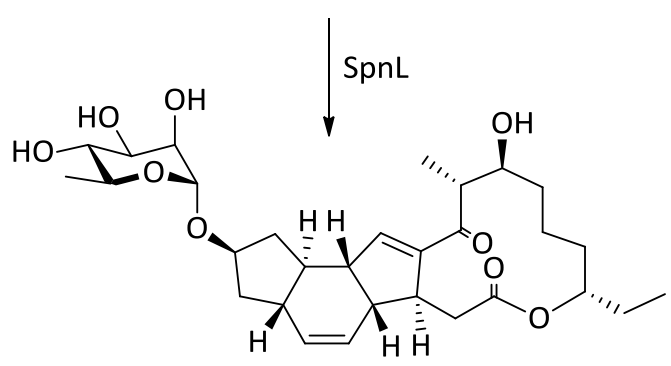

44

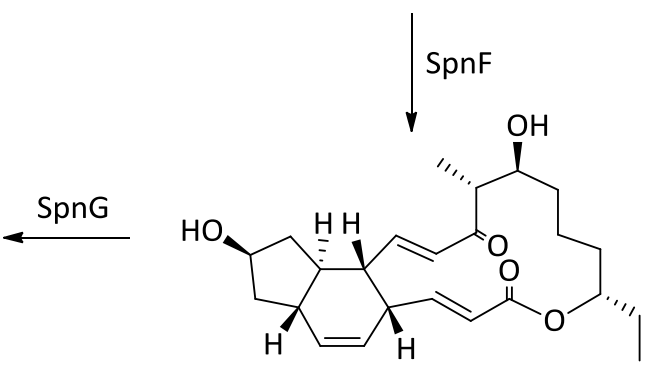

42

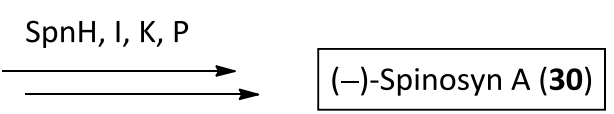

Abbildung 14: $\quad$ Postulierter Biosyntheseweg von Spinosyn A (30) nach Liu et al.

Nach vielen kontroversen Veröffentlichungen über den Mechanismus zum Aufbau des tetracyclischen Grundgerüsts konnten darüber hinaus vier weitere Gene, SpnJ, SpnF, SpnL und SpnM, identifiziert werden, die für die Cyclisierungs-Tranformationen zum Aufbau des tetracyclischen Grundgerüsts verantwortlich sind. Ausgehend vom Produkt $\mathbf{4 0}$ der Polyketid-Synthase katalysiert das Genprodukt von SpnJ, eine Flavinabhängige Dehydrogenase, die selektive Oxidation der C-15-Alkoholfunktion unter Ausbildung des Keto-Intermediats 41. Zur Vorbereitung auf die eigentliche Cyclisierungsreaktion erfolgt anschließend eine Dehydratation unter Eliminierung der Hydroxyfunktion an C-11. Der nachfolgende Schlüsselschritt der Cyclisierungssequenz ist eine transannulare [4+2]-Cycloaddition, welche durch das von SpnF codierte Enzym katalysiert wird und zu dem tricyclischen Zwischenprodukt 42 führt (Abbildung 14). Es konnte dabei aber nicht abschließend geklärt werden, ob es sich bei dieser Reaktion um eine Diels-Alder-Reaktion (katalysiert durch eine DielsAlderase) oder um eine schrittweise erfolgende [4+2]-Cycloaddition handelt. Als 
nächstes erfolgt die selektive Glykosidierung des tricyclischen Makrolactons $\mathbf{4 2}$ mit der L-Rhamnose, bevor SpnL den Cyclisierungsprozess durch Verknüpfung von C-3 und C-14 vollendet. Es wird vermutet, dass es sich hierbei um eine vinyloge MoritaBaylis-Hillman-Reaktion, auch unter dem Namen Rauhut-Currier-Reaktion bekannt, handelt. $^{44}$

Für die Permethylierung der Rhamnoseeinheit sind weitere drei Gene verantwortlich. SpnH, SpnI und SpnK exprimieren jeweils Methyltransferasen, deren Mechanismen mit denen bisher literaturbekannten Methyltransferasen übereinstimmen. Die Spinosynbiosynthese wird durch die abschließende Glykosidierung mit D-Forosamin vollendet. Gesteuert wird diese Reaktion durch das Glykosyltransferasegen SpnP. Die Aufklärung des Biosynthesewegs stellt einen Meilenstein in der über 20 Jahre andauernden Geschichte der Spinosyn-Forschung dar. Mit dem Verständnis der biochemischen Prozesse könnte es nun möglich sein, neue, hochpotente Spinosynanaloga zu entwickeln sowie die Fermentationsprozesse zu optimieren, um die Wirkstoffproduktion zu erhöhen.

\subsubsection{Totalsynthesen von Spinosyn A}

Chemische Totalsynthesen können vor allem im Hinblick auf die Herstellung von Wirkstoffderivaten ein hilfreiches Werkzeug sein. Auch bei der Aufklärung von Absolutkonfigurationen spielen sie noch heute eine gewichtige Rolle. Ziel ist es immer, eine möglichst kurze, selektive und effiziente Totalsynthese zu entwickeln.

Für die Naturstoffklasse der Spinosyne gibt es bis heute lediglich drei bekannte Totalsynthesen. Dies ist sicherlich vor allem der Größe und Komplexität des Moleküls mit seiner großen Anzahl an stereogenen Zentren geschuldet. Die erste Totalsynthese eines Spinosyns wurde 1993 von Evans et al. mit der Herstellung von (+)-Spinosyn A (ent-30) beschrieben. ${ }^{45}$ 


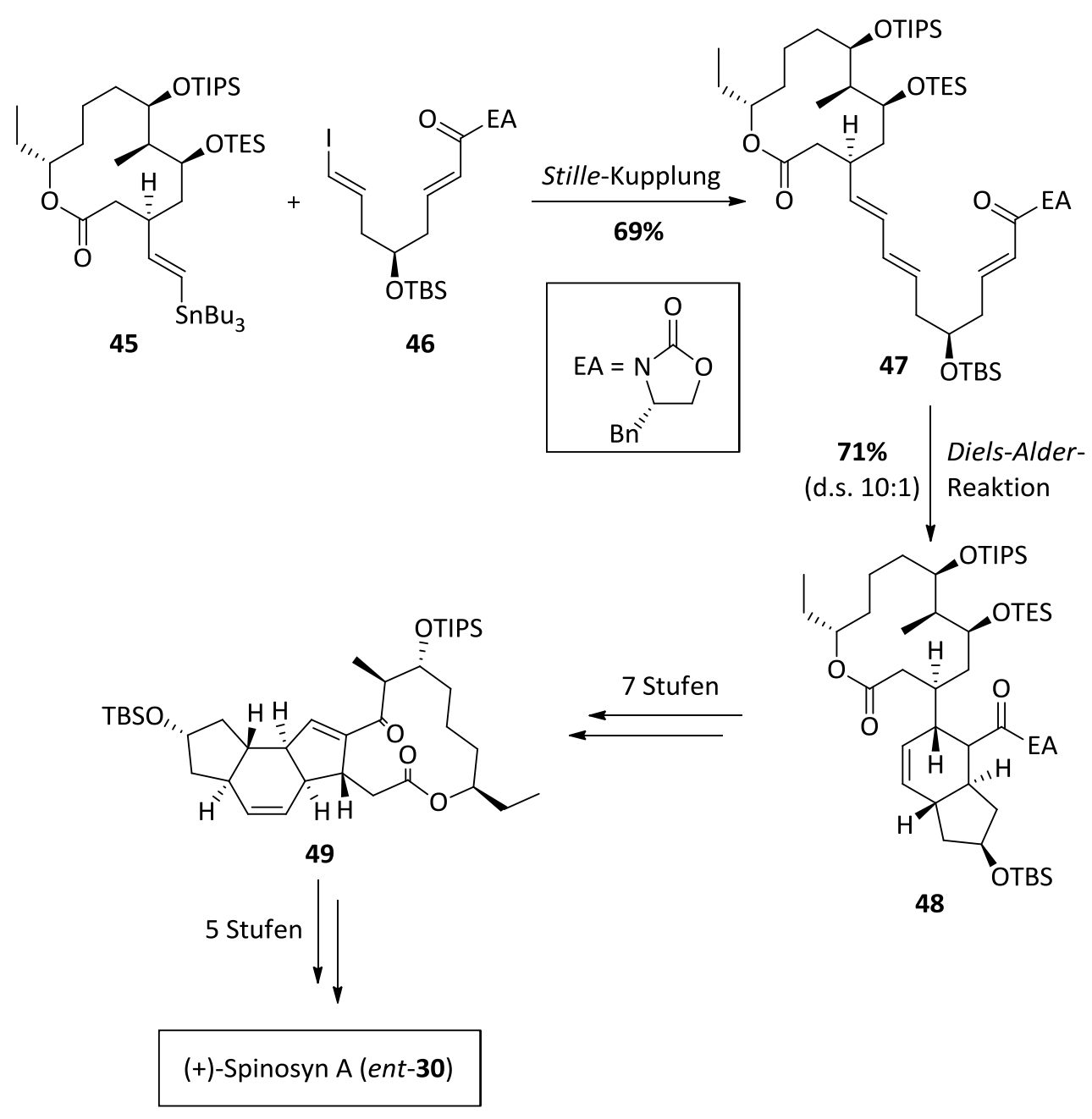

Abbildung 15: $\quad$ Schlüsselschritte der (+)-Spinosyn A (ent-30) Synthese nach Evans et al.

Ein Schlüsselschritt dieser Synthese war eine intermolekulare Stille-Kupplung des Vinyliodids 46 mit dem cyclischen Fragment 45 (Abbildung 15). Diese lieferte den Vorläufer 47 für die darauffolgende intramolekulare Diels-Alder-Reaktion, deren Produkt 48 in sieben weiteren linear geführten Schritten, unter anderem in einer Aldolkondensation, in den Tetracyclus 49 überführt werden konnte. Die Entfernung der Schutzgruppen und die Einführung der Zuckereinheiten ergaben schließlich das nicht natürliche Isomer (+)-Spinosyn A (ent-30). Die Synthese war ein wichtiger Bestandteil der Strukturaufklärung der Spinosyne, da deren Stereochemie zu diesem Zeitpunkt noch nicht aufgeklärt war.

Die erste Totalsynthese des natürlichen Enantiomers (-)Spinosyn A (30) wurde 1998 von Paquette et al. veröffentlicht (Abbildung 16). ${ }^{46}$ 


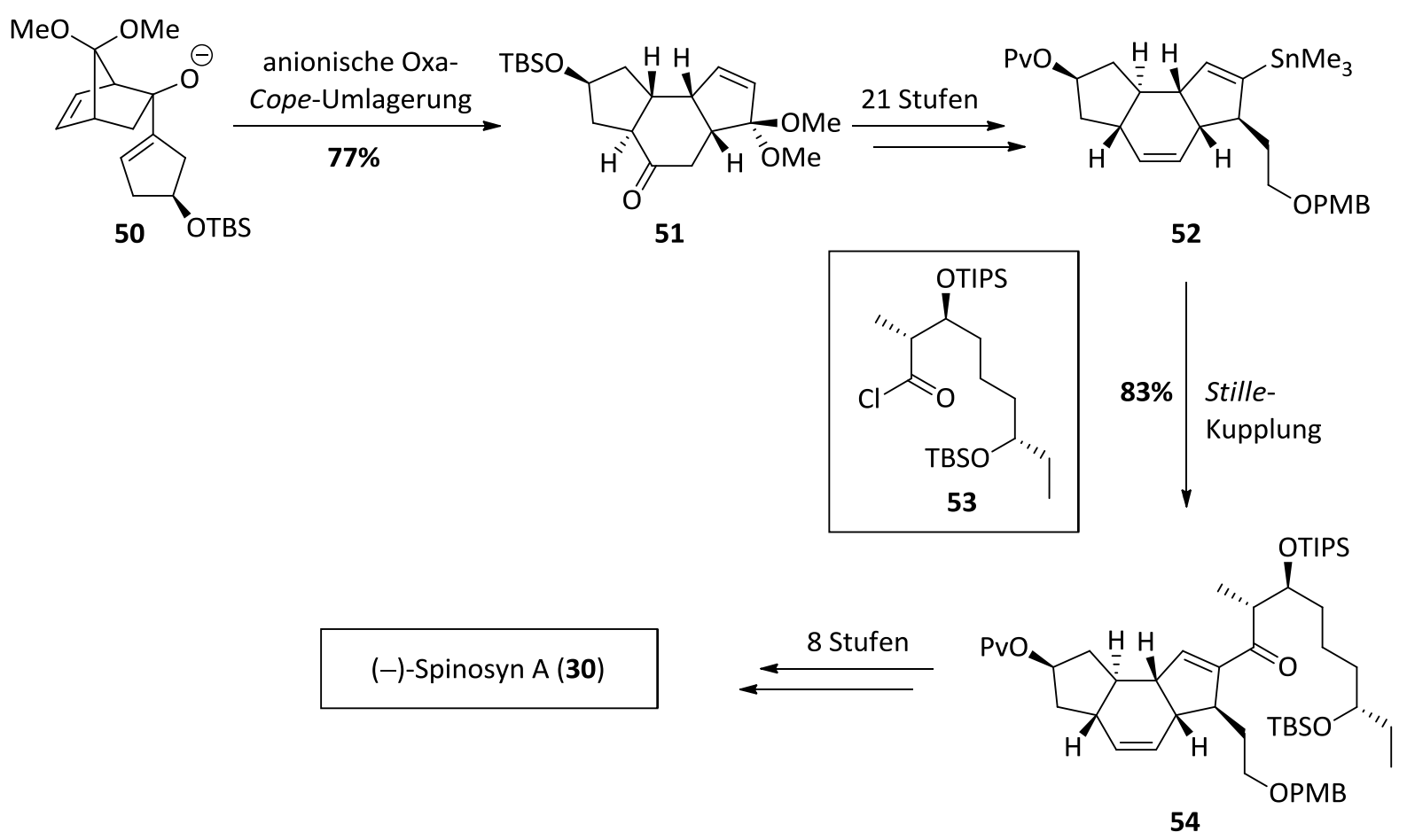

Abbildung 16: Schlüsselschritte der (-)-Spinosyn A (30) Synthese nach Paquette et al.

Eine unter basischen Bedingungen geführte anionische Oxa-Cope-Umlagerung wurde, ausgehend vom enantiomerenreinen Baustein 50, für den Aufbau der as-Indaceneinheit verwendet. Allerdings wies das anellierte System $\mathbf{5 1}$ nicht die den Spinosynen entsprechende stereochemische Verknüpfung der Carbocyclen auf. In 21 linear geführten Schritten war es jedoch möglich, den Tricyclus 52 zu erhalten, der die entsprechende Verknüpfung des Naturstoffs besitzt. Eine Stille-Kupplung mit dem Säurechlorid 53 lieferte den Vorläufer 54 für die Makrolactonisierung. Die Makrolactonisierung miteinbezogen, wurden weitere acht Stufen für die Fertigstellung der Synthese benötigt.

Der dritte totalsynthetische Ansatz wurde 2004 von Roush et al. ${ }^{47}$ publiziert und war durch die Spinosynbiosynthese inspiriert. ${ }^{42}$ Es wurde zunächst der acyclische Vorläufer 55 hergestellt, der über eine Wittig-Horner-Reaktion cyclisiert werden sollte. Unerwartet kam es jedoch unter den verwendeten Bedingungen zu einer Domino-Reaktion, ${ }^{48}$ bei der sich die transannulare Diels-Alder-Reaktion in situ an die Makrolactonisierung anschloss und den Tricyclus 56 bildete (Abbildung 17). Es folgte eine Morita-Baylis-Hillman-Reaktion, durch die der Tetracyclus $\mathbf{5 7}$ in beeindruckender 
Weise in nur zwei Schritten; ausgehend vom acyclischen Baustein $\mathbf{5 5}$ erhalten werden konnte.<smiles>CCO[C@H](CCC[C@H](CC)OC(=O)C[PH](=O)(OCC)OCC)[C@@H](C)C(=O)/C=C/C=C/C[C@H](C/C=C(Br)/C=C/C=O)O[R6]c1ccccc1</smiles>

Rham $=2,3,4-$ Tri-O-methyl- $\alpha$-L-rhamnopyranosyl
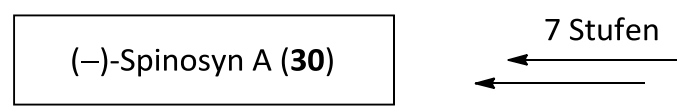

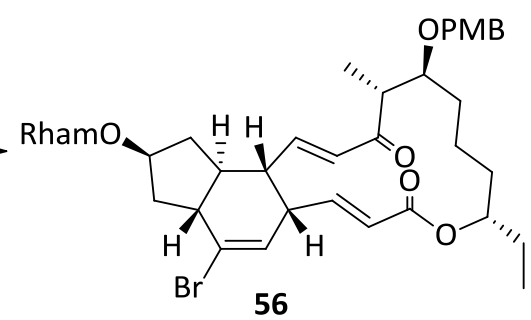

\begin{tabular}{l|l} 
quant. & Morita-Baylis-
\end{tabular} (3 Isomere88:7:5) Hillman-Reaktion

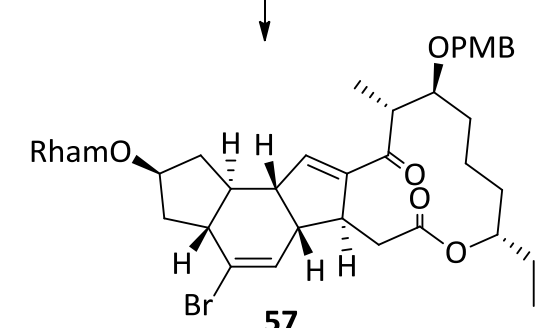

Abbildung 17: $\quad$ Schlüsselschritte der (-)-Spinosyn A (30) Synthese nach Roush et al. 


\section{Zielsetzung und Planung der Arbeit}

Im vorangegangenen Kapitel wurde die noch junge Naturstoffklasse der Spinosyne bereits ausführlich vorgestellt. Ihr größter Vorteil als Insektizide besteht sicherlich in ihrer hohen Selektivität und Effizienz gegenüber einer Vielzahl an Schadorganismen kombiniert mit einem hervorragenden ökologischen Profil. Diese Eigenschaften erweckten im Rahmen eines modernen Pflanzenschutzes großes Interesse von Seiten der agrochemischen Industrie. Darüber hinaus werden die Spinosyne seit kurzem auch zum Schutz von Haus- und Nutztieren vor dem Befall von blutsaugenden Insekten und anderen Parasiten erfolgreich eingesetzt.

Seit der Identifizierung des bakteriellen Produzenten im Jahre $1986^{20}$ und der nachfolgenden Charakterisierung der für die insektizide Wirkung hauptsächlich verantwortlichen Metabolite Spinosyn A (30) und D (31) (Abbildung 18) wurden auf deren Basis zahlreiche neue Pflanzenschutzmittel entwickelt und weltweit zugelassen. Der bis heute ausschließlich durch aufwändige Fermentation gewonnene Wirkstoff dieser Mittel nennt sich Spinosad. Dieser ist das Gemisch der beiden Spinosyne A (30) und D (31) im Verhältnis 85:15, wie es auf natürlichem Wege von Saccharopolyspora spinosa produziert wird.

Im Jahr 2000 traten jedoch bei vereinzelten Schädlingsklassen erste Resistenzen auf. $^{34,35}$ Die Aufgabe der akademischen wie auch der industriellen Forschung sollte nun darin bestehen, im Rahmen eines modernen Resistenzmanagements neue wirksame Spinosynderivate zu entwickeln. Bei der Herstellung solcher Verbindungen nimmt die chemische Synthese eine große Rolle ein. In der Literatur sind bis jetzt erst wenige totalsynthetische Zugänge zu den Spinosynen beschrieben. Diese sind zudem für eine industrielle Anwendung wenig geeignet, da sie zum einen sehr viele Reaktionsschritte aufweisen und zum anderen nur im begrenzten Maße Möglichkeiten für Derivatisierungen bieten. Hinzu kommt, dass die natürlichen Spinosyne alleine am tetracyclischen Grundgerüst neun stereogene Zentren aufweisen, deren selektiver Aufbau, besonders bei der Herstellung im industriellen Maßstab, nicht einfach und mit hohen Kosten verbunden ist. Daher ist es im Hinblick auf Wirtschaft- 
lichkeit und Effizienz unabdingbar, im Zuge der Synthese von Analoga die natürliche Struktur der Spinosyne zu vereinfachen.

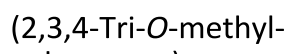

L-rhamnose)
(D-Forosamine)

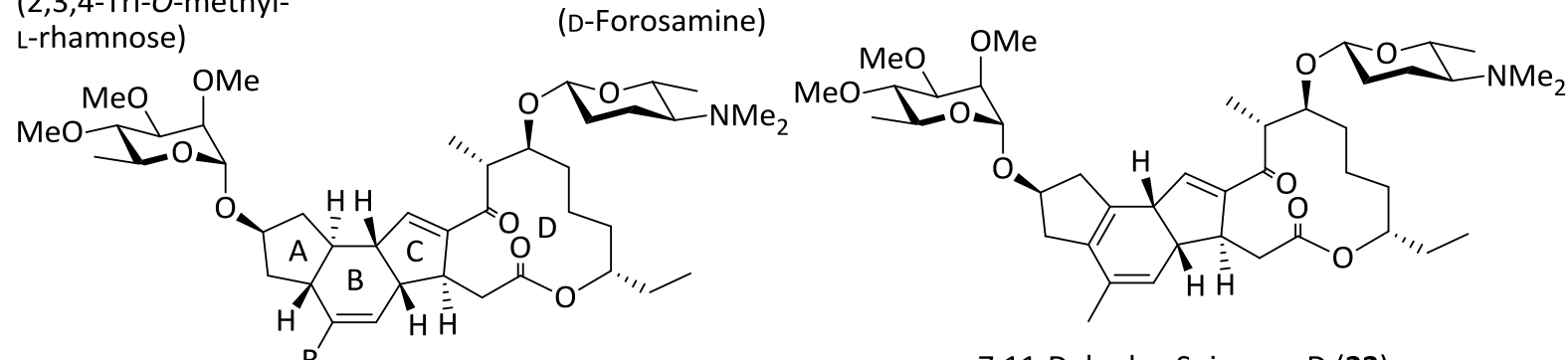

7,11-Dehydro-Spinosyn D (33)

$\mathrm{R}=\mathrm{H}: \quad(-)$-Spinosyn A (30) $\quad\left(\mathrm{LD}_{50}=0.3 \mathrm{ppm}\right)$

$\mathrm{R}=\mathrm{CH}_{3}$ : (-)-Spinosyn D (31) $\quad\left(\mathrm{LD}_{50}=0.8 \mathrm{ppm}\right)$ $\left(\mathrm{LD}_{50}=0.2 \mathrm{ppm}\right)$

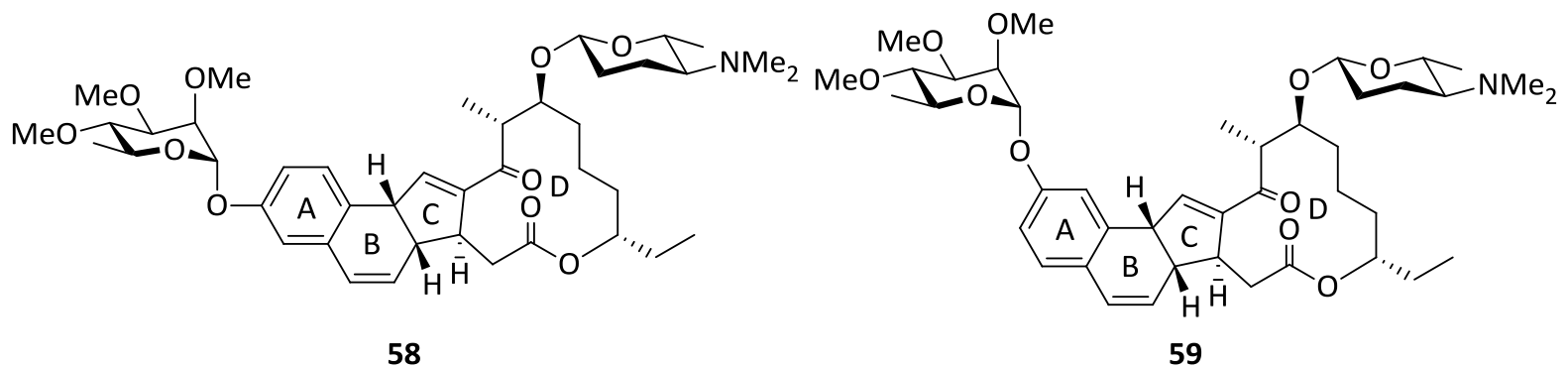

Abbildung 18: Natürliche Spinosyne A (30) und D (31), Spinosynderivat 33 sowie geplante Analoga 58 und 59.

Um herauszufinden, ob eine geplante Modifikation sinnvoll ist oder nicht, sind Struktur-Aktivitäts-Beziehungen von höchster Bedeutung. Im vorigen Kapitel wurden hierzu schon einige Ergebnisse beschrieben. Das 7,11-Dehydroanalogon 33 z. B. zeigt trotz fehlender Stereoinformation an C-7 und C-11 sogar eine geringfügig höhere Aktivität im Vergleich zu den Spinosynen A (30) und D (31). Auf Grundlage dieser Untersuchungen werden im Arbeitskreis Tietze Spinosyn-Analoga vom Typ $\mathbf{5 8}$ und $\mathbf{5 9}$ entwickelt (Abbildung 18). Hier wird die Rhamnose-tragende Cyclopentaneinheit (Ring A) der natürlichen Vorbilder durch einen aromatischen 6-Ring ersetzt. Durch die Einsparung von drei stereogenen Zentren wird die Komplexität des Moleküls entscheidend verringert. Die besondere synthetische Herausforderung dieser Analoga besteht zum einen im Aufbau der cis-Verknüpfung der Ringe $B$ und $C$ sowie in der Anordnung der C=C-Doppelbindungen, zum anderen in der selektiven Einführung der beiden für die Wirkung essentiellen Zuckereinheiten. In vorangegangenen Arbeiten zur Synthese von Spinosynanaloga konnte im Arbeitskreis Tietze 
das tricyclische Grundgerüst bereits mehrfach mit verschiedenen Bausteinen über zwei hintereinander geschaltete Heck-Reaktionen hoch selektiv aufgebaut werden. ${ }^{18}$

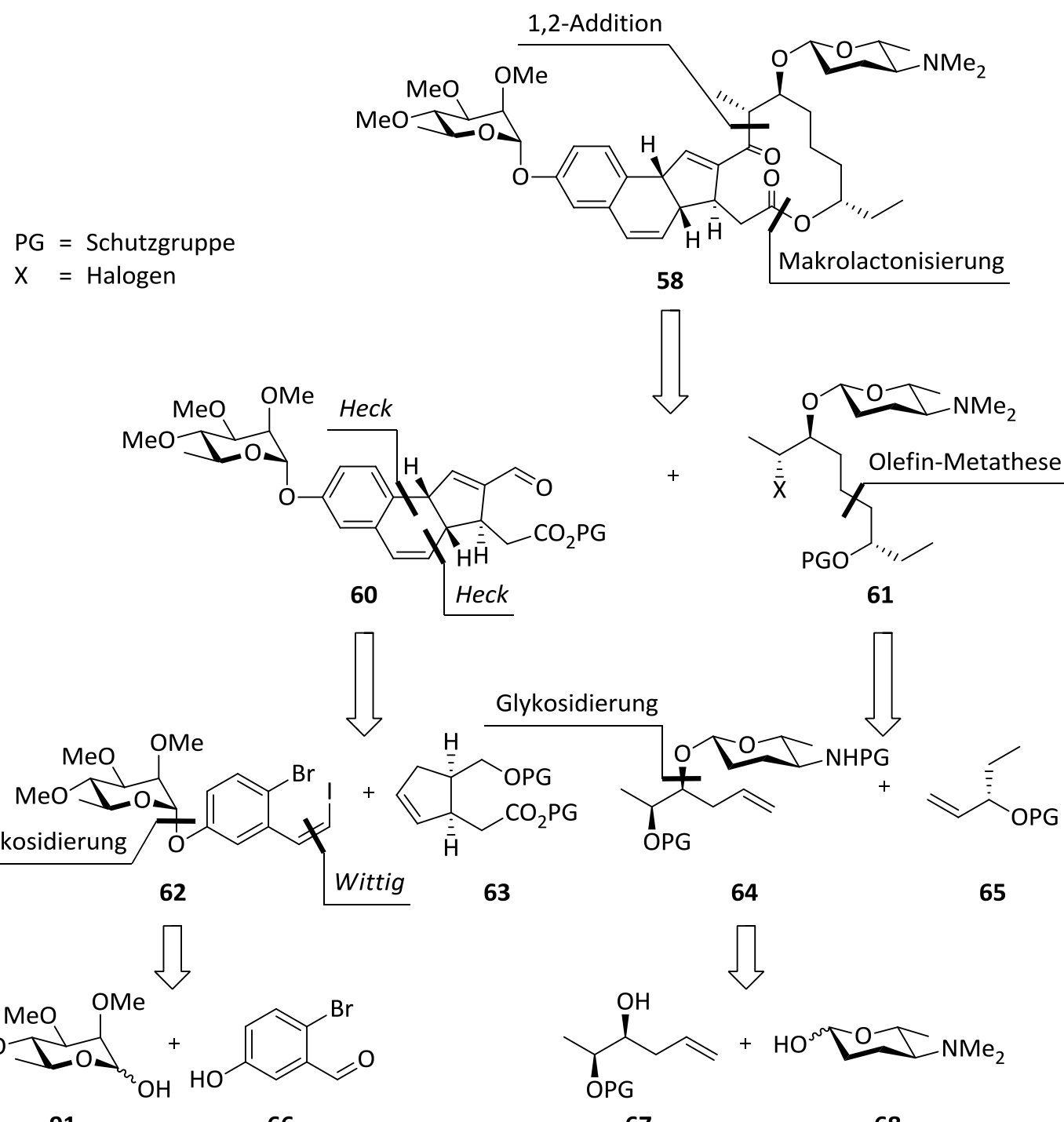

91

66

67

68

Abbildung 19: $\quad$ Retrosyntheseschema zum Aufbau des Spinosynanalogons 58.

Im Rahmen dieser Dissertation sollten die für die Synthese des Spinosynanalogons 58 benötigten Bausteine $\mathbf{6 0}$ und $\mathbf{6 1}$ hergestellt sowie erste Untersuchungen zu deren Kupplung durchgeführt werden (Abbildung 19). Darüber hinaus sollte in Kooperation mit S. Dietz aus dem Arbeitskreis Tietze die $\beta$-selektive Glykosidierung des Bausteins 61 und seiner Vorläufer mit dem von ihr synthetisierten enantiomerenreinen D-Forosamin (68) und Forosaminderivaten untersucht werden. Hier war es wichtig, die Forosamineinheit zu einem möglichst frühen Zeitpunkt der Synthese einzuführen, da sich in früheren Arbeiten herausgestellt hatte, dass eine späte Glyko- 
sidierung entweder gar nicht oder nur in schlechten Ausbeuten realisiert werden konnte. $^{18}$

Im Mittelpunkt stand zunächst die Synthese des Tricyclus 60 unter Verwendung der in früheren Arbeiten etablierten zweifachen Heck-Reaktion. Dazu mussten das cis1,2-disubstituierte Cyclopentenderivat 63 und der aromatische Baustein 62 hergestellt werden. Der aromatische Baustein 62 sollte über die Glykosidierung des Phenols 66 mit 2,3,4-Tri-O-methyl-L-rhamnose (91) und anschließender WittigReaktion zugänglich sein.

Zum Aufbau des $\beta$-Glykosids $\mathbf{6 1}$ bot sich eine Olefin-Metathese zwischen dem enantiomerenreinen C-5-Fragment 65 und dem bereits die Forosamineinheit tragenden Hexen 64 an. Im Anschluss daran sollte eine Methode entwickelt werden, den C-9Baustein 61 mit dem Tricyclus 60 zu kuppeln. Dazu war zunächst eine 1,2-Addition des Glykosids 61 als Lithiumorganyl geplant. Durch eine abschließende Makrolactonisierung sollte dann die Synthese eines Spinosynanalogons vom Typ 58 erfolgen.

Zusammenfassend ergaben sich folgende Teilaufgaben:

- Synthese eines cis-disubstituierten Cyclopentenbausteins vom Typ 63

- Synthese des mehrfach funktionalisierten Aromaten 62

- Aufbau des tricyclischen Grundgerüsts 60 mit cis-verknüpfter B-C-Einheit durch zweifache Heck-Reaktion der Bausteine 62 und 63

- Synthese des $\beta$-Glykosids 61 durch Metathese der Bausteine 64 und 65

- Untersuchungen zur $\beta$-selektiven Glykosidierung mit enantiomerenreinem D-Forosamin (68) in Zusammenarbeit mit S. Dietz aus dem Arbeitskreis Tietze

- Untersuchungen zur Kupplung der Bausteine 60 und 61 zum Aufbau des Spinosynanalogons 58. 


\section{DARSTELLUNG DER ERGEBNISSE}

\section{Synthese des cis-disubstituierten Cyclopentenbausteins rac-69}

\subsection{Mechanistischer Exkurs}

Das Grundgerüst der Spinosynanloga stellt Tricyclus 73 dar, dessen Ringe B und C, wie in Abbildung 20 gezeigt, eine cis-Verknüpfung aufweisen. Der Aufbau des entsprechenden Tricyclus sollte über eine zweifache Heck-Reaktion erfolgen. Um dies zu erreichen, ist es notwendig, einen 1,2-cis-disubstituierten Cyclopentenbaustein bereitzustellen. Der Grund dafür soll kurz anhand mechanistischer Überlegungen erläutert und mit Hilfe der folgenden Abbildung verdeutlicht werden.

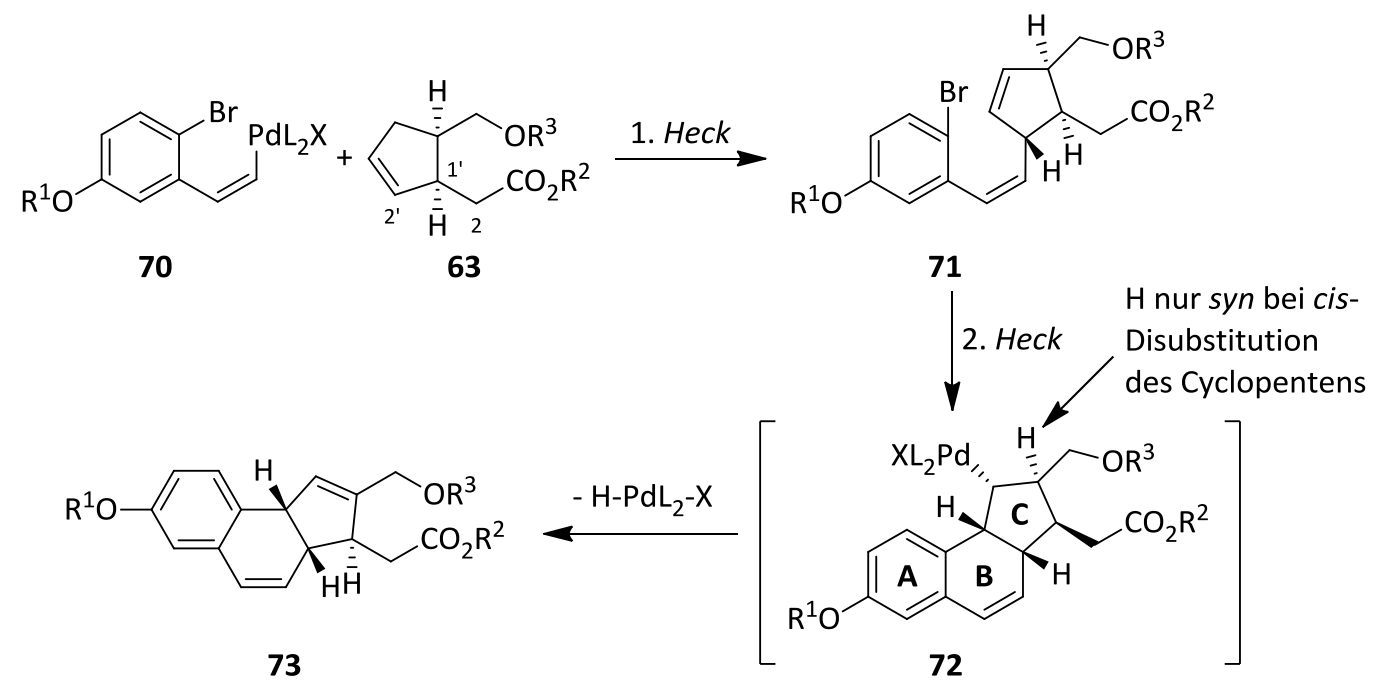

Abbildung 20: $\quad$ Syntheseschema der zweifachen Heck-Reaktion.

Wenn man annimmt, dass der Insertionsschritt regioselektiv an C-2' des Cyclopentenderivats verläuft und es sich um eine diastereoselektive Reaktionssteuerung des stereogenen Zentrums an C-1' handelt, sollte der Angriff des Olefins $\mathbf{6 3}$ an den Palladiumkomplex $\mathbf{7 0}$ von der konkaven Seite erfolgen. Dies führt im ersten Reaktionsschritt dazu, dass ausschließlich das Heck-Kupplungsprodukt $\mathbf{7 1}$ gebildet werden sollte (Abbildung 20). Auch bei der im zweiten Reaktionsschritt folgenden intramolekularen Heck-Reaktion sollte der Insertionsprozess der olefinischen Doppelbindung in den neu gebildeten Pd-Komplex aufgrund der eingeschränkten Flexibilität des Systems erneut von oben erfolgen. Das Ergebnis des angenommenen Reaktions- 
verlaufs ist die Bildung einer intermediären Spezies $\mathbf{7 2}$ mit der gewünschten cisVerknüpfung der Ringe B und C. Von hier aus ist eine Weiterreaktion zum angestrebten tricyclischen Grundgerüst 73 nur dann möglich, wenn ein zum Palladium syn-ständiges Wasserstoffatom in $\beta$-Position für den Eliminierungsprozess vorhanden ist. Diese Anordnung ist wiederum nur bei der Verwendung eines cis-disubstituierten Cyclopentenderivats zu realisieren.

\subsection{Synthese des Bicyclus rac-79}

Eine als Intermediat geeignete Verbindung zur Herstellung eines disubstituierten Cyclopentens mit cis-Anordnung der Substituenten wird in der Literatur von Goldstein et al. $^{49}$ beschrieben. Der Bicyclus 79 (Abbildung 21) lässt sich in wenigen Schritten in ein für die zweifache Heck-Reaktion benötigtes Cyclopentenderivat überführen. Die Herstellung des racemischen Bicyclus $\mathbf{7 9}$ erwies sich bereits in früheren Arbeiten ${ }^{50}$ aus dem Arbeitskreis Tietze als sehr zuverlässig und konnte problemlos in größerem Maßstab (25-50 g) durchgeführt werden.

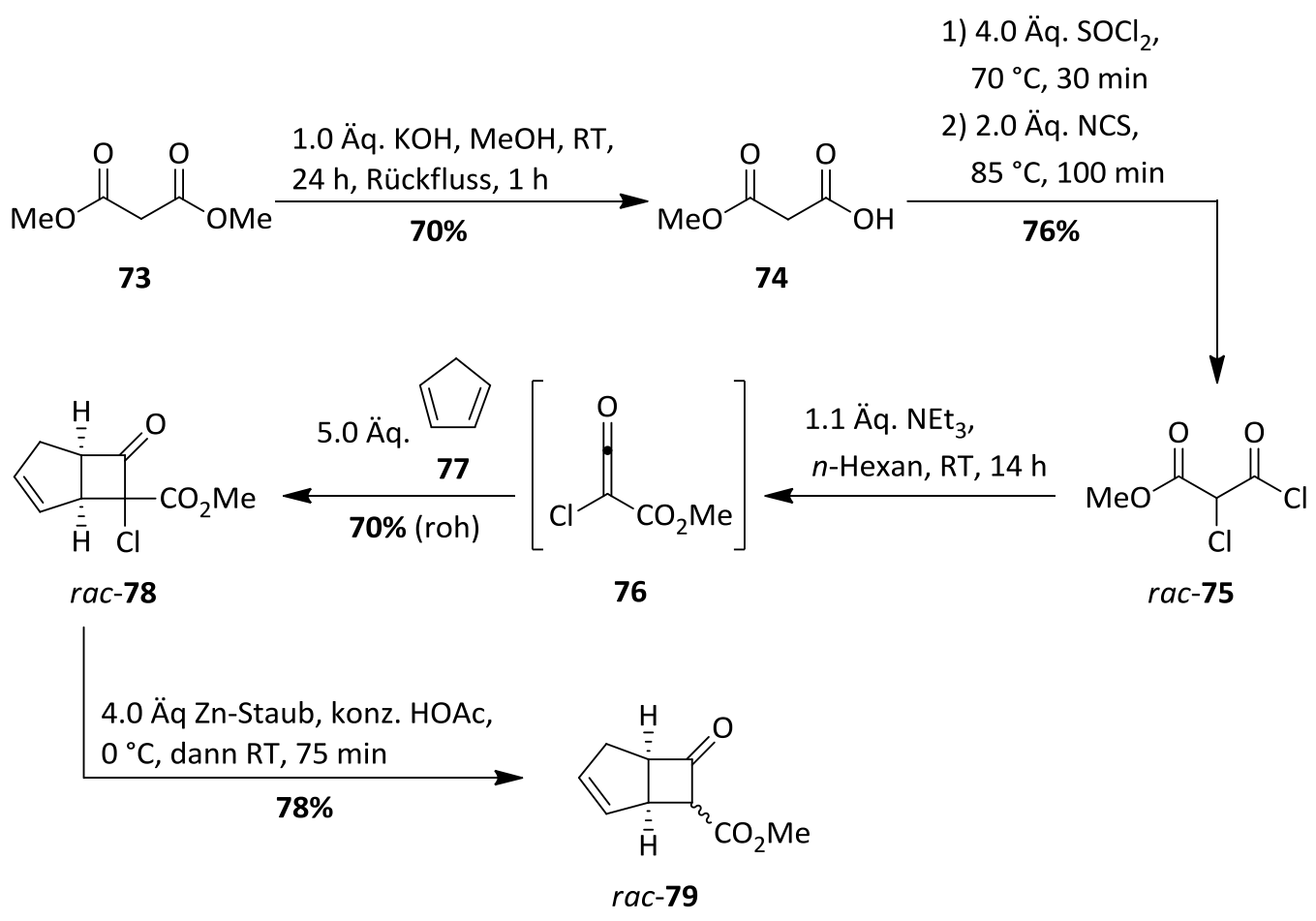

Abbildung 21: $\quad$ Synthese des Bicyclus rac-79.

Die Ausgangsverbindung für die vierstufige Synthesesequenz zur Darstellung des Bicyclus 79 war der kommerziell erhältliche Malonsäuredimethylester (73). Um den 
Monoester $\mathbf{7 4}$ zu erhalten, wurde zunächst selektiv eine der beiden Esterfunktionen gespalten. Dies gelang unter Verwendung von äquimolaren Mengen $\mathrm{KOH}$ in $\mathrm{MeOH}$ bei Raumtemperatur mit einer Ausbeute von $70 \% .{ }^{51}$ Nun wurde zunächst die freie Säurefunktion mit $\mathrm{SOCl}_{2}$ in das entsprechende Säurechlorid überführt, um dann in demselben Reaktionsschritt die Methylengruppe durch Zugabe von N-Chlorsuccinimid unter Erhalt von 75 einfach zu chlorieren. ${ }^{52}$ Dabei war es von großer Bedeutung, die angegebenen Reaktionsbedingungen genau zu befolgen, um einen unselektiven Reaktionsverlauf zu vermeiden.

Um Ausbeuteverlusten vorzubeugen, wurde das reaktive Malonsäurederivat 75 unmittelbar nach Destillation im nächsten Reaktionsschritt mit $\mathrm{NEt}_{3}$ und frisch destilliertem Cyclopentadien (77) umgesetzt. Dabei wurde in situ das Keten 76 gebildet, welches mit dem Dien in einer [2+2]-Cycloaddition unter Bildung des gewünschten Bicyclus 78 reagierte. Die von Woodward und Hoffmann entwickelten Regeln zur Vorhersage des Verlaufs von pericyclischen Reaktionen beschreiben für die Reaktion von Ketenen mit C-C-Doppelbindungen einen thermisch möglichen, da antarafacial erlaubten, konzertierten Reaktionsverlauf, welcher die cis-Verknüpfung der beiden Carbocyclen in 78 gewährleistet. ${ }^{53}$ Der Bicyclus wurde in einer Rohausbeute von $70 \%$ erhalten und konnte ohne weitere Reinigung im nächsten Reaktionsschritt eingesetzt werden.

Um die von Goldstein et al. beschriebene stark gesundheitsgefährdende, radikalische Dechlorierung mit $\mathrm{HSnBu}_{3}$ und $\mathrm{AIBN}$ in Benzol zu vermeiden, ${ }^{49}$ wurde im nächsten Schritt eine Dehalogenierung mit Zinkstaub in Eisessig durchgeführt. ${ }^{54}$ Mit dieser weitaus milderen Variante konnte die entsprechende dechlorierte Spezies 79 nach nur $20 \mathrm{~min}$ bei $0{ }^{\circ} \mathrm{C}$ und destillativer Reinigung in einer guten Ausbeute von $78 \%$ erhalten werden. Laut spektroskopischer Befunde fiel das Produkt dabei als 2:1 Diastereomerengemisch an.

Betrachtet man die Einführung des Chloratoms im zweiten Schritt der Sequenz, so erscheint sie auf den ersten Blick vor dem Hintergrund einer Entfernung im letzten Schritt als überflüssig. Die Literatur verweist jedoch darauf, dass eine Cycloaddition 
des entsprechend nicht chlorierten Ketens mit Cyclopentadien (77) nicht möglich ist. $^{49}$

\subsection{Synthese des Cyclopentens rac-69}

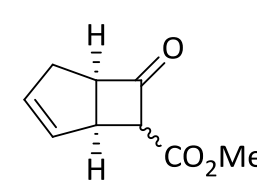

rac-79

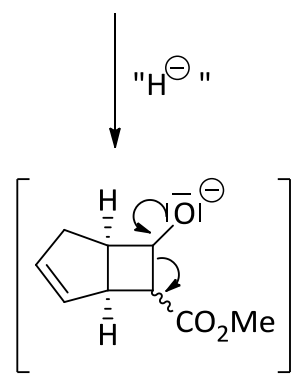

80

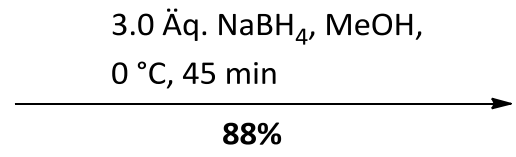

$88 \%$
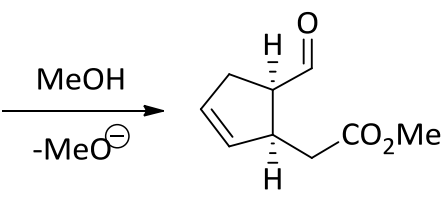

rac-81

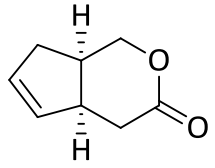

rac-83

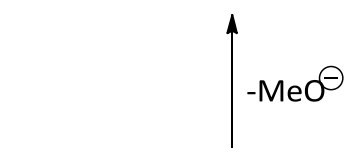

82

Abbildung 22: Postulierte Domino-Sequenz zur Synthese des Lactons 83

Im nächsten Schritt sollte das bicyclische Intermediat $\mathbf{7 9}$ in das Lacton $\mathbf{8 3}$ überführt werden. Dies gelang in einer Domino-Sequenz unter Verwendung von 3.0 Äq. $\mathrm{NaBH}_{4}$ in Methanol (Abbildung 22). Dabei kam es zunächst zu einer Reduktion der Ketofunktion, gefolgt von einer Retroaldoladdition, was zur intermediären Bildung des cis-Aldehyds $\mathbf{8 1}$ führte. Das nächste Äquivalent $\mathrm{NaBH}_{4}$ reduzierte die neugebildete Aldehydfunktion umgehend und es wurde das Alkoholat $\mathbf{8 2}$ gebildet. Den Abschluss der Domino-Sequenz stellt der intramolekulare Angriff des Alkoholations auf die Esterfunktion dar. Dieser erfolgte aufgrund der cis-Anordnung der beiden Substituenten und ihrer daraus resultierenden räumlichen Nähe äußerst schnell, so dass die Bildung möglicher Nebenprodukte weitgehend unterdrückt wurde. Das gewünschte Lacton 83 konnte in einer sehr guten Ausbeute von 88\% erhalten werden.

Als nächstes sollte nun das Lacton $\mathbf{8 3}$ geöffnet werden und die neu entstandene Säurefunktion als tert-Butylester geschützt werden. Dabei setzen allerdings zahlreiche Darstellungsvarianten die Verwendung von starken Brønsted- oder LewisSäuren voraus, ${ }^{55}$ was jedoch ohne vorherige Schützung der primären Hydroxyfunktion unweigerlich zur Rückbildung des Lactons 83 aus dem Natriumsalz 84 der 
Carbonsäure führen würde. Eine Alternative wäre die Verwendung einer aktivierten Carboxylfunktion. ${ }^{55}$ Aber auch hier wäre eine vorherige Schützung der Alkoholfunktion unabdingbar, da die Aktivierung sonst ebenfalls in dem Erhalt des Lactons $\mathbf{8 3}$ enden würde. Eine praktikable Lösung dieses Problems stellte die Verwendung des aus $N, N^{\prime}$-Diisopropylcarbodiimid (86) zugänglichen Isoharnstoffs 87 dar. ${ }^{56}$ Dieser ermöglichte unter leicht sauren Bedingungen eine Übertragung des tert-Butylfragments auf das Natriumcarboxylat 84 (Abbildung 23).

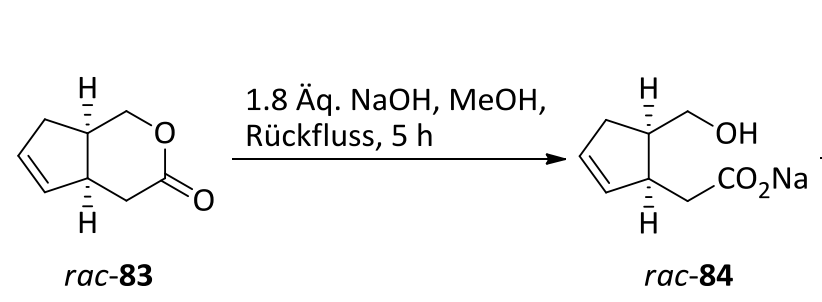

1) 3.0 Äq. $\mathrm{NH}_{4} \mathrm{Cl}, 3.4$ Äq. 87

2) 1.5 Äq. $\mathrm{NH}_{4} \mathrm{Cl}, 3.4$ Äq. 87

3) $1.5 \ddot{\mathrm{Aq}}$. $\mathrm{NH}_{4} \mathrm{Cl}, 3.4$ Äq. 87, $\mathrm{CH}_{2} \mathrm{Cl}_{2} / t$-BuOH (1:1), RT, $19 \mathrm{~h}$

79\% (über 2 Stufen)

rac-83

rac-84

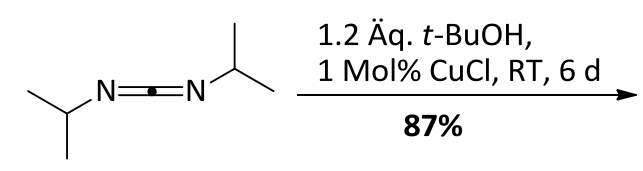

86

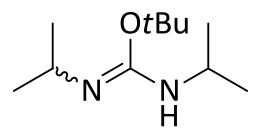

87

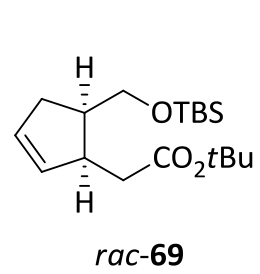

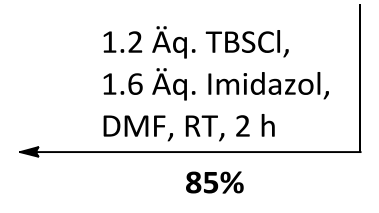

$85 \%$

Abbildung 23: $\quad$ Synthese des cis-disubstituierten Cyclopentenderivats 69.

Die Synthese des Isoharnstoffs $\mathbf{8 7}$ gelang in sehr guten Ausbeuten und war auch im Multigrammmaßstab (100 g) möglich. Neben dem relativ hohen Anschaffungspreis für das Startmaterial (100 $\mathrm{g} \approx 180 €$ ) stellte die lange Reaktionszeit von $6 \mathrm{~d}$, die den Isoharnstoff $\mathbf{8 7}$ in $\mathbf{8 7 \%}$ lieferte, das einzig nennenswerte Problem dar. Dieser musste bei der Umsetzung von rac-84 in großem Überschuss (10.2 Äq.) eingesetzt werden, um das nach basischer Hydrolyse vorliegende Natriumcarboxylat in den gewünschten tert-Butylester 85 zu überführen. Die besten Ausbeuten von 79\% über 2 Stufen wurden hierbei erzielt, wenn der Isoharnstoff $\mathbf{8 7}$ sequentiell in definierten Zeitabständen zugeben wurde. ${ }^{18 c}$ Ferner konnte zusätzlich nicht umgesetztes Lacton 83 reisoliert werden. Der Versuch eines Austauschs des teuren Startmaterials $N, N^{\prime}$-Diiso- 
propylcarbodiimid (86) durch das wesentlich günstigere Dicyclohexylcarbodiimid war nicht erfolgreich.

Der letzte Schritt der Synthesesequenz hin zum für die Heck-Reaktion benötigten cisdisubstituierten Cyclopentenbaustein stellte eine TBS-Schützung der primären Alkoholfunktion in Verbindung 85 dar. Dies gelang ohne Schwierigkeiten nach einem StandardprotokolI ${ }^{55}$ mit TBSCl und Imidazol in DMF. Das gewünschte Produkt 69 konnte nach einer Reaktionszeit von $2 \mathrm{~h}$ bei Raumtemperatur in guter Ausbeute von 85\% erzielt werden. Bei der Synthese des benötigten cis-disubstituierten Cyclopentenbausteins 69 konnte auf diverse Vorarbeiten aus dem Arbeitskreis Tietze zurückgegriffen werden. ${ }^{18 c}$

\subsection{Diskussion ausgewählter spektroskopischer Daten der Verbindung rac-69}

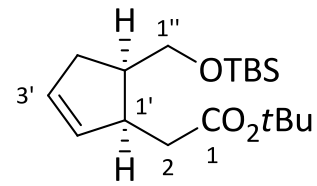

In Abbildung 24 ist das ${ }^{1} \mathrm{H}$-NMR-Spektrum (300 MHz, $\mathrm{CDCl}_{3}$ ) der Verbindung 69 mit der entsprechenden Zuordnung der Signale dargestellt. Die drei Singuletts bei $\delta=0.02$, 0.86 und $1.43 \mathrm{ppm}$ können den drei Methylgruppen des tert-Butylester $(\delta=1.43 \mathrm{ppm})$ und der tert-Butyldimethylsilylschutzgruppe (Me: $\delta=0.02 \mathrm{ppm}$, tert-Bu: $\delta=0.86 \mathrm{ppm}$ ) der primären Alkoholfunktion zugeordnet werden.

Weitere charakteristische Signale in Form von vier Dubletts vom Dublett werden für die jeweils diastereotopen Protonen an C-2 und C-1" erhalten. Hierbei weisen die Signale für die diastereotopen Protonen an C-2 eine deutlich unterschiedliche chemische Verschiebung auf. Das $2-\mathrm{H}_{\mathrm{A}}$-Proton resoniert bei $\delta=2.06 \mathrm{ppm}$ und besitzt für die vicinale Kopplung mit dem benachbarten 1'-H eine Kopplungskonstante von $J=9.9 \mathrm{~Hz} .2-\mathrm{H}_{\mathrm{B}}$ resoniert hingegen bei $\delta=2.45 \mathrm{ppm}$ mit einer vicinalen Kopplungskonstanten von $J=6.2 \mathrm{~Hz}$. Die den beiden Protonen gemeinsame Kopplungskonstante nimmt mit $J=15.0 \mathrm{~Hz}$ einen für geminale Kopplungen typischen Wert an. Ein wesentlich geringerer Unterschied in der chemischen Verschiebung zeigt sich für die beiden diastereotopen Protonen an C-1". 


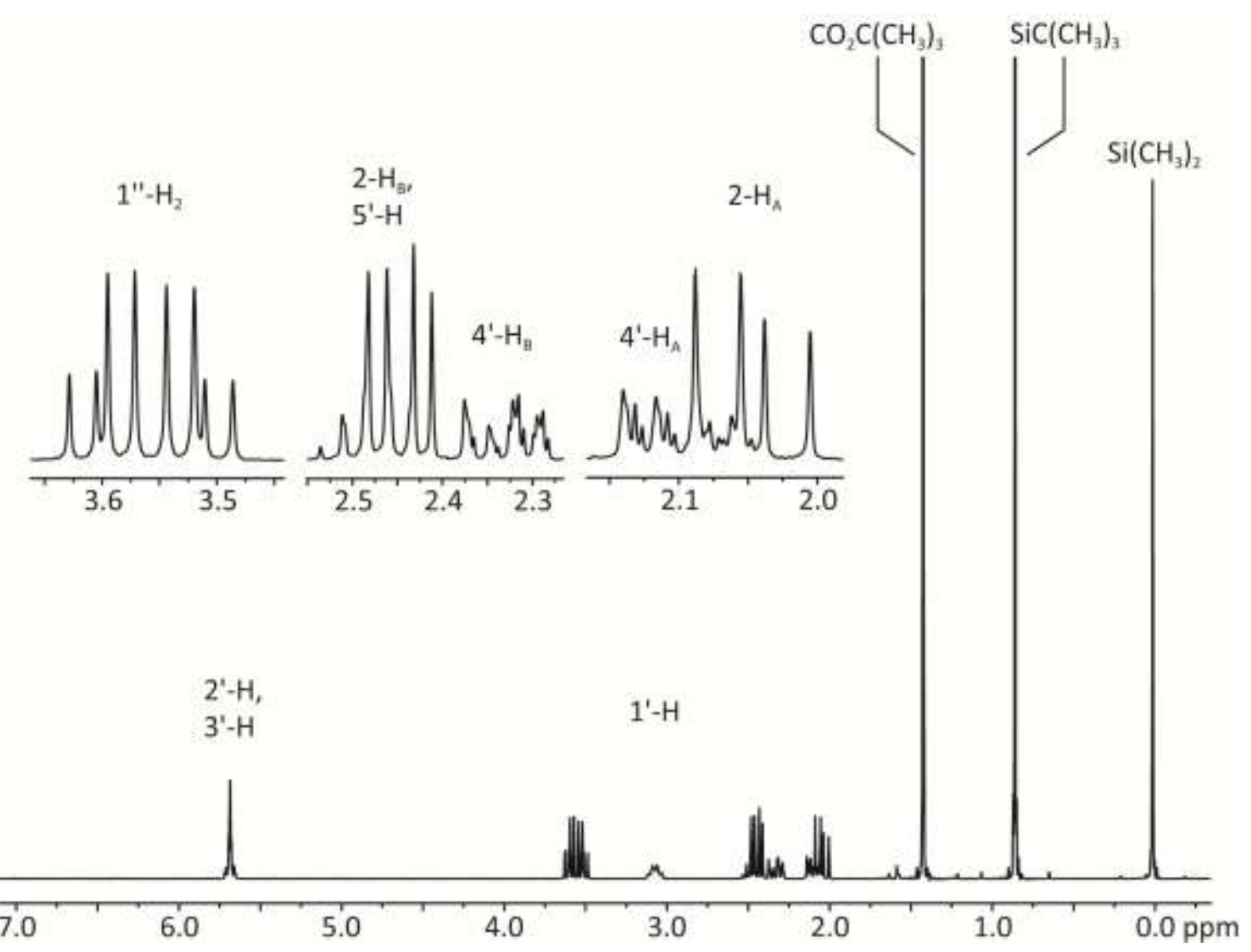

Abbildung 24: $\quad{ }^{1} \mathrm{H}-\mathrm{NMR}\left(300 \mathrm{MHz}, \mathrm{CDCl}_{3}\right)$ der Verbindung 69.

Bei $\delta=3.52 \mathrm{ppm}$ mit einer geminalen Kopplungskonstanten von $J=10.0 \mathrm{~Hz}$ und einer vicinalen von $J=7.3 \mathrm{~Hz}$ resoniert $1 "-\mathrm{H}_{\mathrm{A}}$. Nur leicht tieffeldverschoben bei $\delta=3.60$ ppm wird mit gleicher geminaler, aber anderer vicinaler Kopplungskonstante $(J=7.1 \mathrm{~Hz})$ das Signal für $1 "-\mathrm{H}_{\mathrm{B}}$ gefunden. Auch die beiden diastereotopen Protonen an $\mathrm{C}-4$ ' resonieren bei leicht unterschiedlichen chemischen Verschiebungen. 4'- $_{A}$ resoniert im Bereich $\delta=2.05-2.16 \mathrm{ppm}$ und $4 "-\mathrm{H}_{\mathrm{B}}$ bei $\delta=2.26-2.38 \mathrm{ppm}$ jeweils in Form eines Multipletts. Das Multiplett im Bereich von $\delta=2.41-2.54 \mathrm{ppm}$ ist dem Proton an C-5' zuzuordnen. Das Proton an C-1' resoniert bei $\delta=2.98-3.19 \mathrm{ppm}$ ebenfalls als Multiplett. Eine Aussage über die cis-Ständigkeit dieser Protonen kann aufgrund der nicht auswertbaren Kopplungskonstanten an dieser Stelle nicht getroffen werden. Jedoch wurde dies bereits durch NOESY-Experimente in früheren Arbeiten $^{18}$ im Arbeitskreis Tietze und durch NOESY-Experimente im späteren Verlauf dieser Arbeit verifiziert. Das letzte noch verbliebene Signal ist ein zentriertes Multiplett mit einer chemischen Verschiebung von $\delta=5.69 \mathrm{ppm}$. Hierbei handelt es sich um die Signale für die beiden olefinischen Protonen $2^{\prime}-\mathrm{H}$ und 3 '-H, die, wie für 
Doppelbindungsprotonen zu erwarten ist, im Tieffeldbereich des Spektrums zu finden sind.

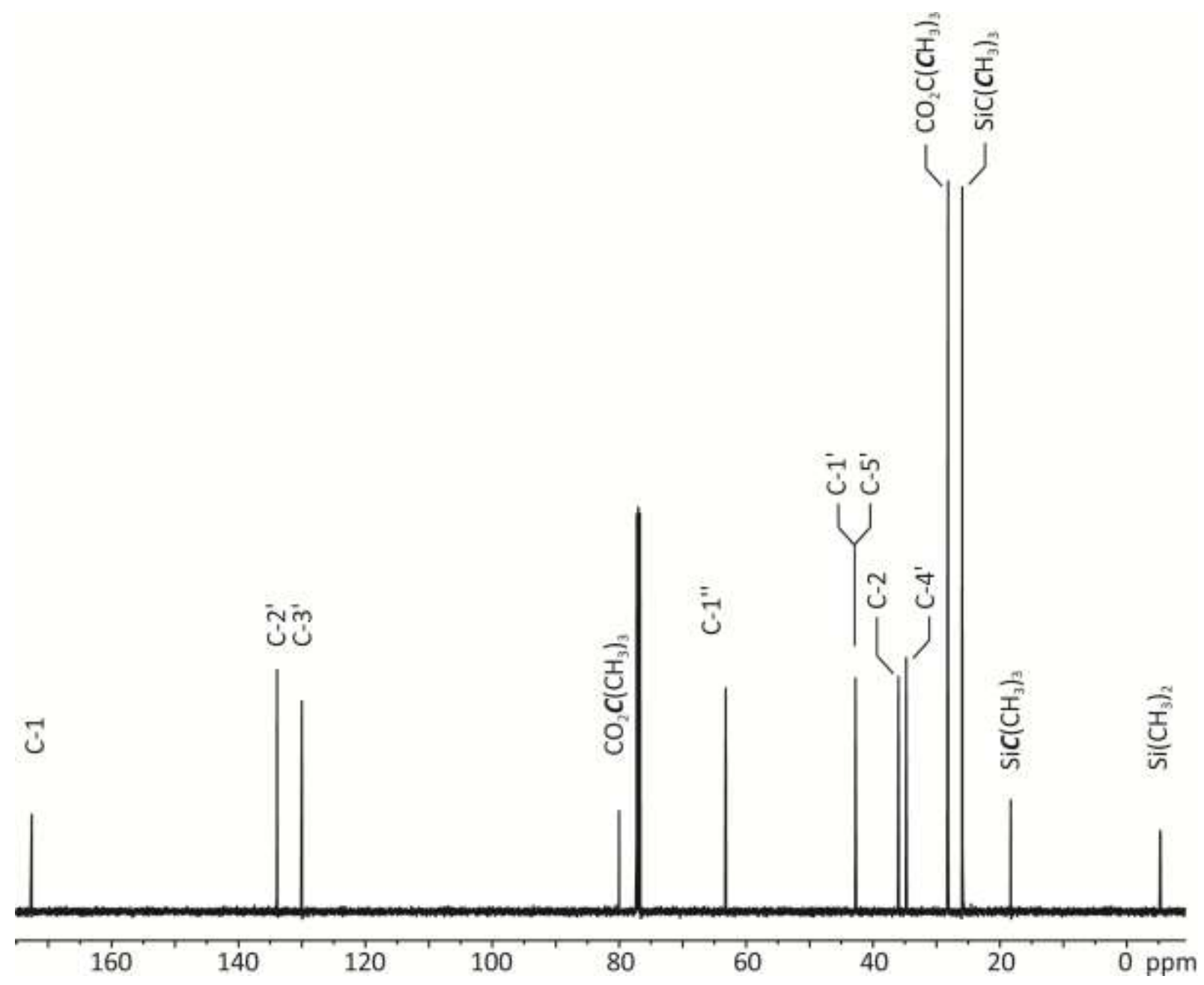

Abbildung 25: $\quad{ }^{13} \mathrm{C}-\mathrm{NMR}-\mathrm{Spektrum}\left(126 \mathrm{MHz} \mathrm{CDCl}_{3}\right.$ ) der Verbindung 69.

Abbildung 25 zeigt das ${ }^{13} \mathrm{C}-\mathrm{NMR}-$ Spektrum $\left(126 \mathrm{MHz}, \mathrm{CDCl}_{3}\right.$ ) der Verbindung 69 mit den zu den einzelnen Signalen gehörenden Zuordnungen. Im Hochfeldbereich des Spektrums findet man zunächst die Signale der TBS-Schutzgruppe. Die Kohlenstoffatome resonieren bei $\delta=-5.3$ und $\delta=-5.2 \mathrm{ppm}\left(\mathrm{Si}\left(\mathrm{CH}_{3}\right)_{2}\right), \delta=18.3 \mathrm{ppm}\left(\mathrm{SiC}\left(\mathrm{CH}_{3}\right)_{3}\right)$ sowie bei $\delta=26.0 \mathrm{ppm}\left(\mathrm{SiC}\left(\mathrm{CH}_{3}\right)_{3}\right)$. Weitere charakteristische Signale lassen sich für die Kohlenstoffatome des tert-Butylesters finden. Diese resonieren bei $\delta=28.2 \mathrm{ppm}$ $\left(\mathrm{CO}_{2} \mathrm{C}\left(\mathrm{CH}_{3}\right)_{3}\right)$ und bei $\delta=80.0 \mathrm{ppm}\left(\mathrm{CO}_{2} \underline{\mathrm{C}}\left(\mathrm{CH}_{3}\right)_{3}\right)$. Das Signal für das C-1-Atom der Esterfunktion kann bei $\delta=172.6 \mathrm{ppm}$ beobachtet werden. Die beiden olefinischen Kohlenstoffatome resonieren bei $\delta=130.0 \mathrm{ppm}\left(\mathrm{C}-3^{\prime}\right)$ und $133.9 \mathrm{ppm}$ (C-2'), was typischen Werten für die chemische Verschiebung von Kohlenstoffatomen einer $\mathrm{C}=\mathrm{C}$ Doppelbindung entspricht. Die Resonanzfrequenzen der übrigen aliphatischen 
Kohlenstoffatome betragen $\delta=34.8 \mathrm{ppm}\left(\mathrm{C}-4^{\prime}\right), 36.0 \mathrm{ppm}(\mathrm{C}-2), 42.7$ und $42.8 \mathrm{ppm}$ (C-1', C-5') sowie $\delta=62.2$ ppm für das C-1"-Atom.

\section{Synthese des aromatischen Bausteins 62}

\subsection{Synthese des Glykosyldonors 92}

Die Synthese des Glykosyldonors 92 wurde in Anlehnung an die von A. Grube im Arbeitskreis Tietze entwickelte Vorschrift durchgeführt. Als Ausgangsverbindung für die Synthese des Trichloracetimidats 92 wurde das käuflich erhältliche L-RhamnoseMonohydrat (88) verwendet. Im ersten Schritt der Synthese konnte das Methylpyranosid $\mathbf{8 9 ^ { 5 7 }}$ in quantitativer Ausbeute erhalten werden. Dies gelang durch Umsetzung der Verbindung $\mathbf{8 8}$ mit Amberlite IR-120 (stark saurer lonenaustauscher) unter Rückfluss in Methanol (Abbildung 26).

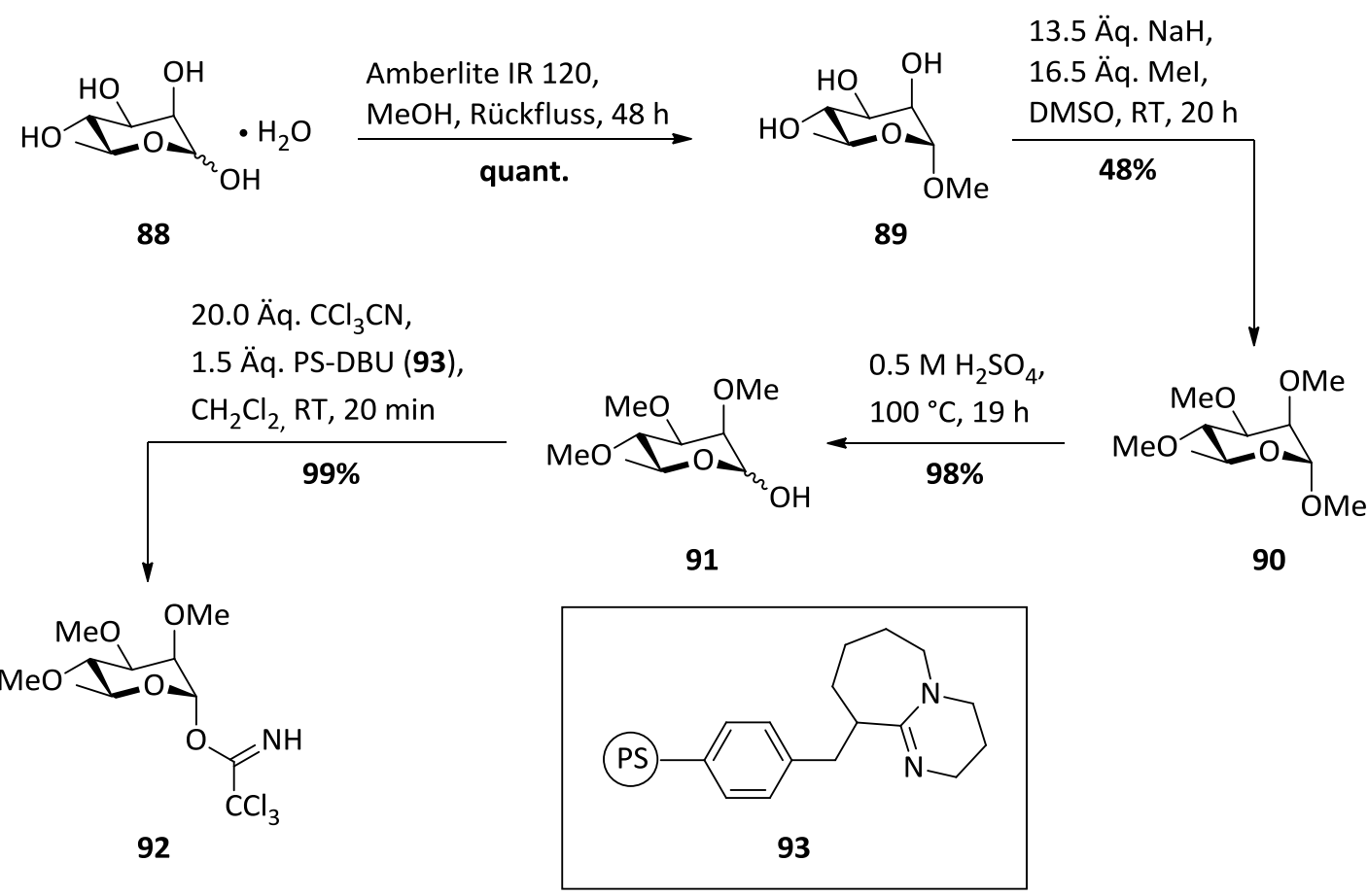

Abbildung 26: $\quad$ Synthese des Glykosyldonors 92.

Die drei übrigen Hydroxyfunktionen wurden dann in nur einem weiteren Schritt mit $\mathrm{NaH}$ und $\mathrm{Mel}$ in DMSO methyliert. ${ }^{58}$ Das vollständig methylierte $\alpha$-Pyranosid 90 wurde jedoch lediglich in moderater Ausbeute von 48\% erhalten. Eine direkte globale Methylierung des L-Rhamnose-Monohydrats (88) zu Verbindung 90 führte jedoch zu 
noch schlechteren Ausbeuten und darüber hinaus auch zu einer verstärkten Bildung des nicht gewünschten entsprechenden $\beta$-Anomers.

Eine selektive Entschützung der anomeren Hydroxyfunktion unter sauren Bedingungen führte in $98 \%$ Ausbeute zu Verbindung $91{ }^{58}$ welche als Gemisch der beiden Anomere $(\alpha: \beta=6: 1)$ erhalten wurde.

Für die im späteren Teil der Synthese möglichst selektive $\alpha$-Gykosidierung des L-Rhamnosederivats mit dem Phenol 66 (siehe Abbildung 28) wurde nun eine Abgangsgruppe gesucht, die zu einer hohen $\alpha$-Selektivität führt. Es wurde hierfür auf die in der Literatur bereits mit großem Erfolg eingesetzte Trichloracetimidat-Gruppe zurückgegriffen. Diese von Schmidt et al. ${ }^{59}$ entwickelte Methode kombiniert eine hohe Reaktivität mit einer hervorragenden $\alpha / \beta$-Selektivität. Diese Eigenschaften der Abgangsgruppe sowie der anomere Effekt des Ringsauerstoffatoms und der benachbarten axialen Methoxygruppe sollten unter Einsatz einer starken Lewis-Säure eine selektive $\alpha$-Glykosidierung ermöglichen.

Die abschließende Aktivierung von Verbindung 91 zum Trichloracetimidat 92 gelang unter Verwendung von Polymer-gebundenem DBU $93^{60}$ als Base und Trichloracetonitril in $\mathrm{CH}_{2} \mathrm{Cl}_{2}$ (Abbildung 26). Der Einsatz von Polymer-gebundenem DBU 93 vereinfachte die Aufarbeitung im Vergleich zu herkömmlichem DBU erheblich, da hier lediglich eine Filtration über Celite erforderlich ist und auf eine anschließende säulenchromatographische Reinigung verzichtet werden kann. So wurde das gewünschte $\alpha$-Trichloracetimidat 92 rein und in sehr guter Ausbeute von 99\% erhalten. 


\subsection{Synthese des Phenols 66}

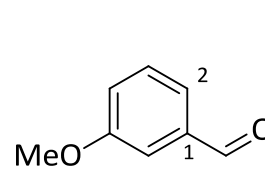

94

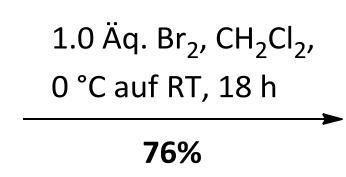

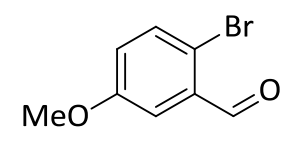

95

$$
77 \% \mid \begin{aligned}
& 2.1 \mathrm{Äq} . \mathrm{BBr}_{3}, \mathrm{CH}_{2} \mathrm{Cl}_{2} \text {, } \\
& -20^{\circ} \mathrm{C}, 22 \mathrm{~h}
\end{aligned}
$$

66

\section{Abbildung 27: $\quad$ Synthese des Phenols 66.}

Das Startmaterial für die Herstellung des als Glykosylakzeptor fungierenden Phenols 66 stellte der sehr günstig erhältliche 3-Methoxybenzaldehyd (94) dar. Dieser musste im ersten Schritt regioselektiv an C-2 bromiert werden. Dies gelang unter Verwendung von elementarem $\mathrm{Br}_{2}$ in $\mathrm{CH}_{2} \mathrm{Cl}_{2}$ bei Raumtemperatur. ${ }^{18 a}$ Nach Umkristallisation aus $n$-Pentan konnte die gewünschte bromierte Spezies 95 in 76\% Ausbeute erhalten werden (Abbildung 27). Abschließend erfolgte die Spaltung des aromatischen Methylethers nach einem Standardprotokoll ${ }^{61}$ bei $-20^{\circ} \mathrm{C}$ mit der starken Lewis-Säure $\mathrm{BBr}_{3}$ in $\mathrm{CH}_{2} \mathrm{Cl}_{2}$. Das entsprechende Phenol 66 wurde in guter Ausbeute von $77 \%$ isoliert.

\subsection{Synthese des Vinyliodids 62}

Dank der hohen Reaktivität des Trichloracetimidats 92 konnte die Lewis-Säure-katalysierte Glykosidierung ${ }^{60}$ des Phenols 66 in sehr hohen Ausbeuten von bis zu $88 \%$ realisiert werden.

Nun musste abschließend nur noch der Aldehyd 96 in eine lodvinylfunktionalität überführt werden (Abbildung 28). Dies geschah mit Hilfe einer Wittig-Reaktion unter Verwendung des Phosphoniumsalzes $97^{62}$ in THF, bei der das (Z)-konfigurierte Vinyliodid 62 in einer Ausbeute von 78\% isoliert werden konnte. 
<smiles>COC1C(OC)C(OC)C(OC(=N)C(Cl)(Cl)Cl)C1OC</smiles>

92

$\mathrm{PPh}_{3}+\mathrm{CH}_{2} \mathrm{I}_{2} \underset{60 \%}{\stackrel{\text { Toluol, } 80{ }^{\circ} \mathrm{C}, 14 \mathrm{~h}}{\longrightarrow}}\left[\mathrm{Ph}_{3} \mathrm{PCH}_{2}\right]^{+} \mathrm{I}^{-}$

97

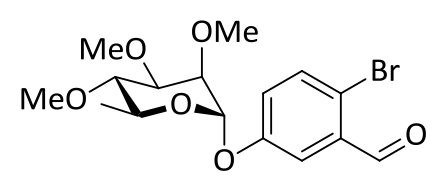

96

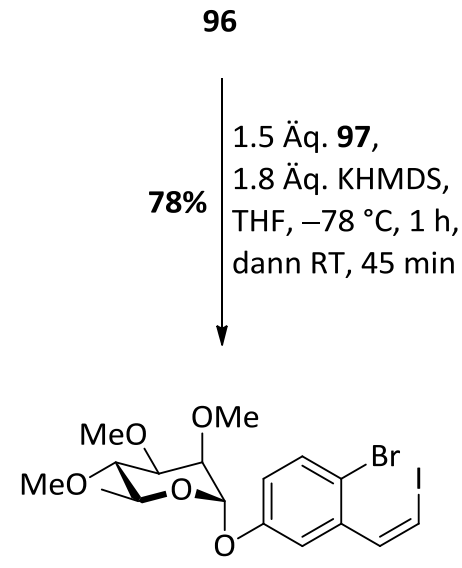

62

Abbildung 28: $\quad$ Synthese des Vinyliodids 62.

Auch die Synthese des für die Heck-Reaktion benötigten aromatischen Fragments 62 geschah in Anlehnung an die von A. Grube aus dem Arbeitskreis Tietze bereits durchgeführten Arbeiten.

\subsection{Diskussion ausgewählter spektroskopischer Daten der Verbindung 62}

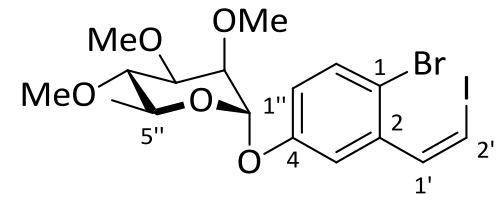

Abbildung 29 zeigt das ${ }^{1} \mathrm{H}-\mathrm{NMR}$-Spektrum (300 MHz, $\mathrm{CDCl}_{3}$ ) von Verbindung 62 mit der entsprechenden Zuordnung der Signale. Im Hochfeldbereich des Spektrums resonieren die drei Protonen des C- 6 " bei $\delta=1.25$ ppm als Dublett und weisen mit einer Kopplungskonstanten von $J=6.2 \mathrm{~Hz}$ einen typischen Wert für eine vicinale Kopplung auf. Für das 4"-H findet man ein Triplett mit einer chemischen Verschiebung von $\delta=3.18 \mathrm{ppm}$, welches dadurch entsteht, dass beide axial-axial-Kopplungen mit den benachbarten Protonen eine gleich große Kopplungskonstante von $J=9.4 \mathrm{~Hz}$ aufweisen. Die Resonanzfrequenzen der drei Methoxygruppen unterscheiden sich nur geringfügig, so dass nur zwei Singuletts bei $\delta=3.54$ und $3.55 \mathrm{ppm}$ beobachtet werden. Dem Multiplett im Bereich von $\delta=3.58-3.68$ ppm können sowohl das 3"-H 
als auch das 5"-H zugeordnet werden. Das Proton an C-2" zeigt eine Resonanzfrequenz von $\delta=3.75$ ppm und tritt in Form eines Dubletts vom Dublett auf.

Die Kopplungskonstanten von $J=3.3$ und $1.9 \mathrm{~Hz}$ stellen typische Werte für eine äquatorial-axial-Kopplung mit 3"-H sowie für eine äquatorial-äquatorial-Kopplung mit 1"-H dar. Das Proton am anomeren Zentrum C-1" zeigt eine starke Verschiebung in den Tieffeldbereich und das Dublett mit einer kleinen Kopplungskonstanten von $J=1.9 \mathrm{~Hz}$ deutet auf das Vorhandensein einer äquatorial-äquatorial-Kopplung hin. Diese beiden Fakten lassen nur den Schluss zu, dass es sich bei der vorliegenden Verbindung um ein $\alpha-G l y k o s i d$ handeln muss.

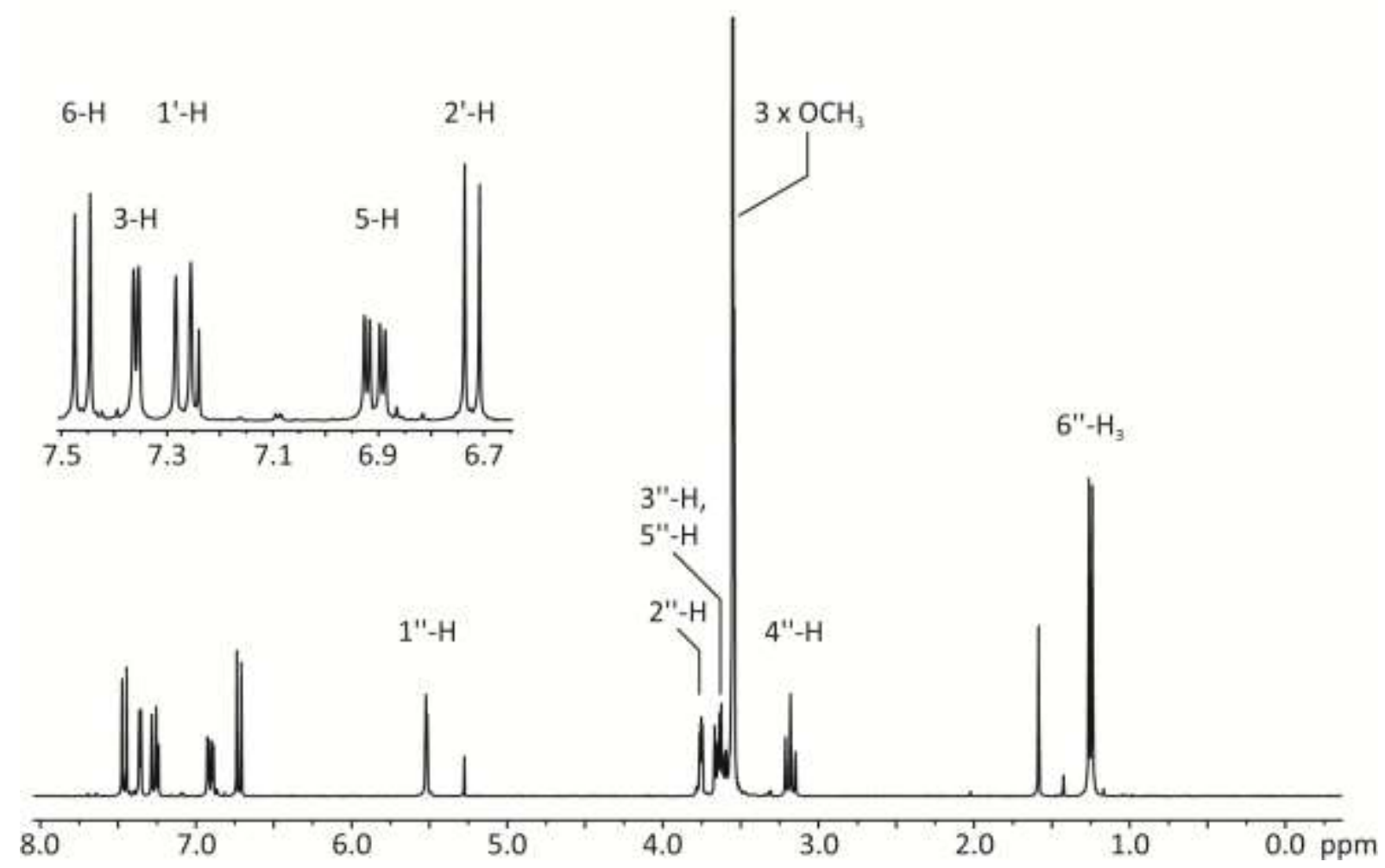

Abbildung 29: $\quad{ }^{1} \mathrm{H}-\mathrm{NMR}-S p e k t r u m\left(300 \mathrm{MHz}, \mathrm{CDCl}_{3}\right)$ der Verbindung 62.

Die aromatischen Signale im Tieffeldbereich zeigen das markante Kopplungsmuster eines 1,2,4-trisubstituierten Aromaten. Das Proton an C-5 resoniert bei $\delta=6.91 \mathrm{ppm}$ in Form eines Dubletts vom Dublett mit einer ortho-Kopplungskonstanten von $J=8.8 \mathrm{~Hz}$ und einer meta-Kopplungskonstanten von $J=2.9 \mathrm{~Hz}$. Das sich dazu in orthoPosition befindende $6-\mathrm{H}$ resoniert bei $\delta=7.46 \mathrm{ppm}$ und erzeugt ein Dublett mit einer für ortho-Kopplungen typischen Kopplungskonstanten von $J=8.8 \mathrm{~Hz}$. Eine metaKopplung $(\mathrm{J}=2.9 \mathrm{~Hz})$ mit $5-\mathrm{H}$ bedingt die Aufspaltung des Protons an $\mathrm{C}-3 \mathrm{zu}$ einem 
Dublett, welches eine chemische Verschiebung von $\delta=7.36 \mathrm{ppm}$ aufweist. Die räumliche Nähe der beiden vinylischen Protonen 1'-H und 2'-H zum aromatischen System bewirkt eine starke Verschiebung in den Tieffeldbereich, so dass 2'-H bei $\delta=6.72 \mathrm{ppm}$ und $1^{\prime}-\mathrm{H}$ sogar bei $\delta=7.27 \mathrm{ppm}$ resoniert. Die identische Kopplungskonstante der beiden Protonen von $J=8.5 \mathrm{~Hz}$ ist ein eindeutiges Zeichen für das Vorliegen einer (Z)-konfigurierten Doppelbindung.

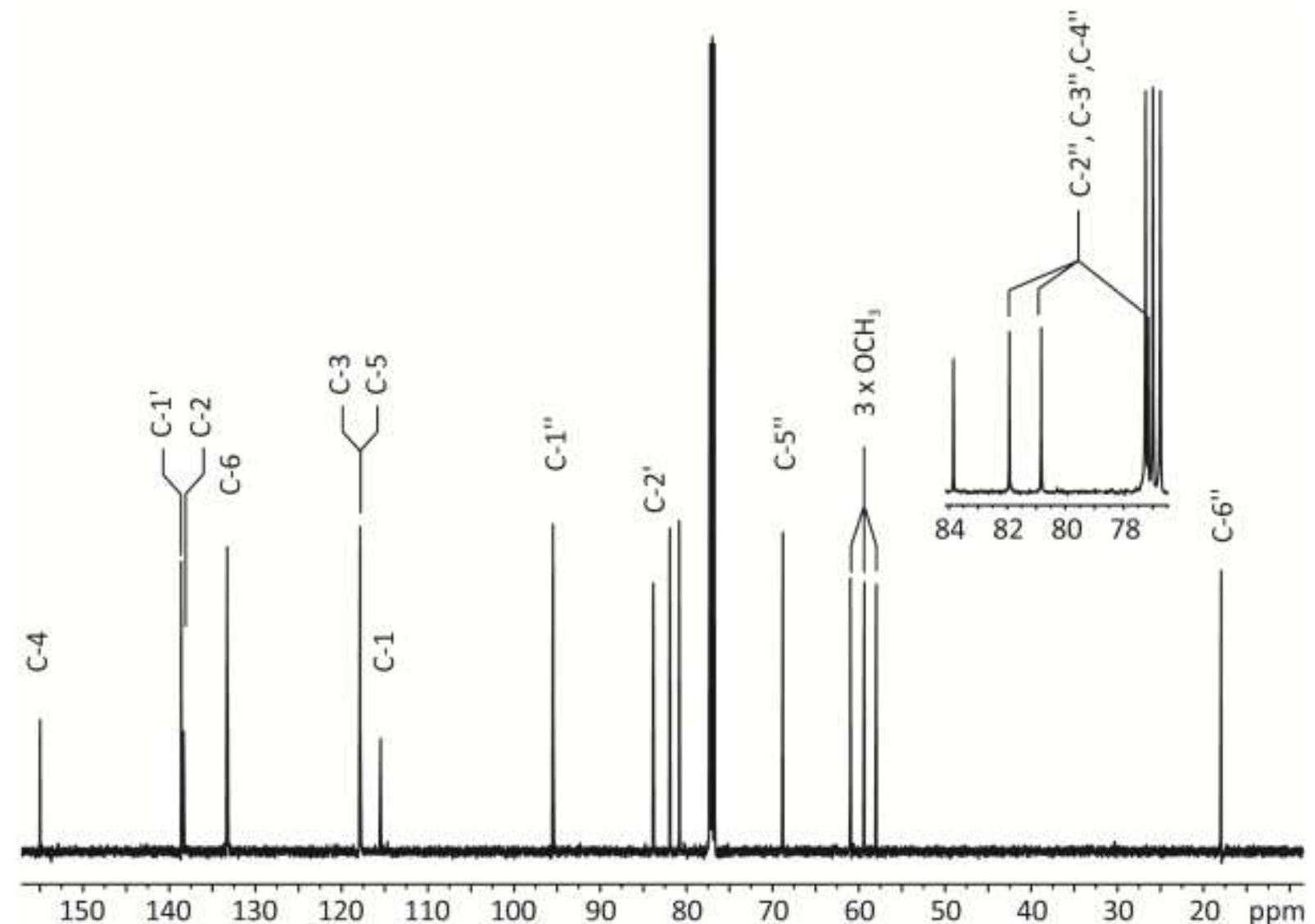

Abbildung 30: $\quad{ }^{13} \mathrm{C}-\mathrm{NMR}-S p e k t r u m\left(126 \mathrm{MHz}, \mathrm{CDCl}_{3}\right)$ der Verbindung 62.

Im ${ }^{13}$ C-NMR-Spektrum des Aromaten 62 (Abbildung 30) resoniert das C-6"-Kohlenstoffatom bei $\delta=18.0 \mathrm{ppm}$. Die drei Methoxygruppen zeigen jeweils eine charakteristische chemische Verschiebung bei $\delta=58.0,59.4$ und $61.0 \mathrm{ppm}$. Das Signal bei $\delta=95.5$ ppm kann eindeutig dem Kohlenstoffatom C-1" des anomeren Zentrums zugeordnet werden. Die übrigen vier Ringkohlenstoffatome der Zuckereinheit resonieren im Bereich zwischen $\delta=68.8$ und 81.9 ppm. Das olefinische C-2' zeigt eine ungewöhnlich starke Verschiebung in den Hochfeldbereich bei $\delta=83.8$. Dies kann durch den Schweratomeffekt des lodsubstituenten erklärt werden. Im Bereich zwischen $\delta=115.5$ und $138.3 \mathrm{ppm}$ resonieren die aromatischen Kohlenstoffatome 
C-1, C-3, C-5, C-6 und C-2. Die Resonanzfrequenz des zweiten olefinischen Kohlenstoffatoms C-1' beträgt $\delta=138.6 \mathrm{ppm}$. Komplettiert wird der Signalsatz durch das am weitesten in den Tieffeldbereich verschobene Signal für C-4 bei $\delta=155.0$ ppm.

\section{Synthese des Tricyclus 60}

\subsection{Inter- und Intramolekulare Heck-Reaktion}

Bei der intermolekularen Kupplung zwischen dem cis-disubstituierten Cyclopentenbaustein 69 mit dem aromatischen Vinyliodid 62 konnte erneut von Vorarbeiten aus dem Arbeitskreis Tietze profitiert werden. Hier wurden in früheren Arbeiten ${ }^{18}$ bereits ausführliche Studien und Optimierungen für die Bedingungen der intermolekularen Heck-Reaktion zwischen einer Vielzahl von Cyclopentenderivaten und Arylvinyliodiden durchgeführt. Aus diesem Grund waren weitergehende Studien für die Umsetzung der vorliegenden Bausteine nicht nötig.

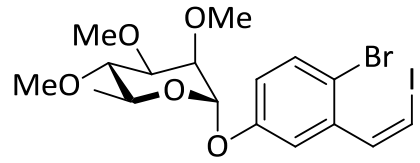

62

$+$

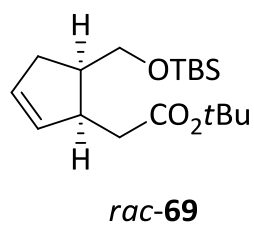

2.5 Äq. 69, $5 \mathrm{Mol} \% \mathrm{Pd}(\mathrm{OAc})_{2}$ 3.0 Äq. NaOAc, 1.0 Äq. $n-\mathrm{Bu}_{4} \mathrm{NCl}$, DMF, RT, $20 \mathrm{~h}$

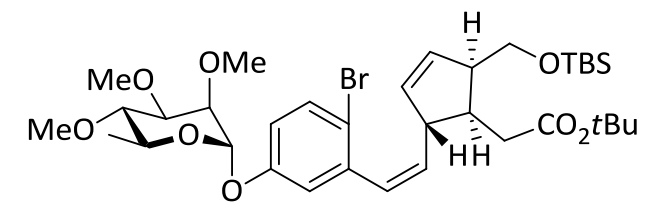

98

+ Diastereomer

$48 \%$

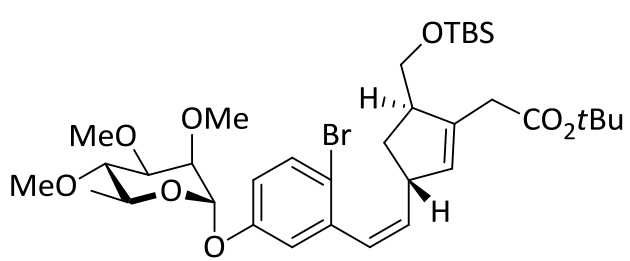

99

+Diastereomer

Abbildung 31: Intermolekulare Heck-Reaktion der Bausteine 62 und 69.

Schließlich wurde die Kupplungs-Reaktion bei Raumtemperatur mit $\mathrm{Pd}(\mathrm{OAc})_{2}$ als Katalysator und unter der Verwendung von $n-\mathrm{Bu}_{4} \mathrm{NCl}$ und $\mathrm{NaOAc}$ als Additive durchgeführt. Das erwünschte Kupplungsprodukt 98 wurde dabei in einer Ausbeute von $48 \%$ erhalten, wobei es sich um das 2:1-Gemisch zweier Diastereomere handelte, da der Cyclopentenbaustein 69 als racemisches Gemisch eingesetzt wurde 
(Abbildung 31). Es wurde primär ein 1:1-Verhältnis der beiden Diastereomere erwartet, da der Rhamnose-Rest als chiraler Induktor verhältnismäßig weit entfernt vom Reaktionszentrum ist. Die Zuordnung der beiden Diastereomere erfolgt auf der Stufe der tricyclischen Verbindung 102. Aufgrund der Übersichtlichkeit und zur Vereinfachung wird jedoch im Laufe dieses Abschnitts lediglich das Hauptdiastereomer abgebildet. Als zusätzliche Nebenprodukte traten darüber hinaus das Regioisomer 99, ebenfalls als 2:1-Gemisch der Diastereomere, sowie ein nicht weiter auftrennbares Gemisch von isomerisierten $(E)$-Kupplungsprodukten auf.

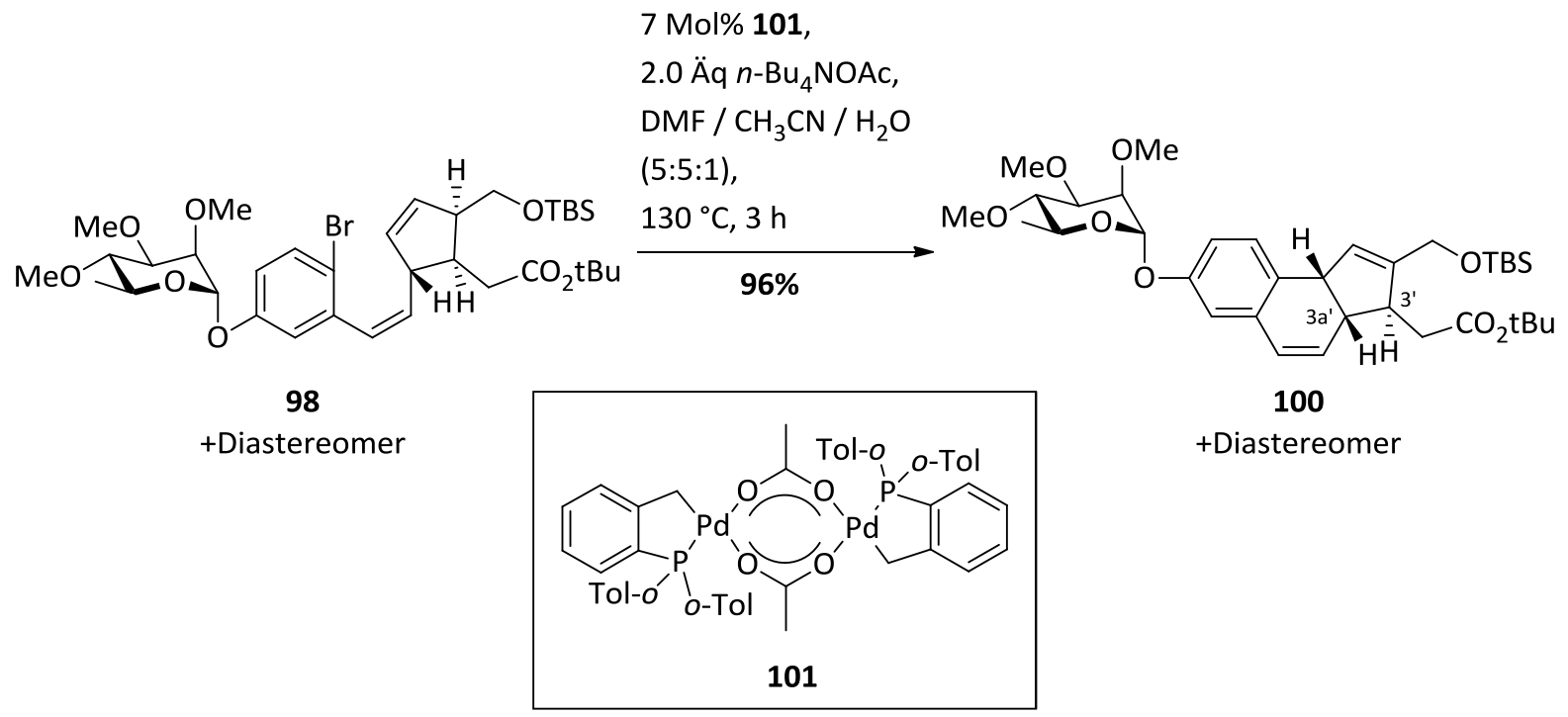

Abbildung 32: $\quad$ Intramolekulare Heck-Reaktion von Verbindung 98.

Das Kupplungsprodukt 98 wurde im folgenden Schritt zum Aufbau des tricyclischen Grundgerüsts in einer intramolekularen Heck-Reaktion verwendet. Die Wahl des Katalysatorsystems für diese Heck-Reaktion wurde durch Vorarbeiten auf verschiedenen Themengebieten im Arbeitskreis Tietze beeinflusst. Die Reaktion ließ sich in 96\% Ausbeute mit dem Palladacyclus 101, auch unter dem Namen Herrmann-BellerKatalysator bekannt, unter Zusatz von $n-\mathrm{Bu}_{4} \mathrm{NOAc}$ in einem Lösungsmittelgemisch aus DMF / $\mathrm{CH}_{3} \mathrm{CN} / \mathrm{H}_{2} \mathrm{O}(5: 5: 1)$ bei $130{ }^{\circ} \mathrm{C}$ durchführen (Abbildung 32).

\subsection{Synthese des Aldehyds 60}

Der nächste Schritt in der Synthesesequenz bestand nun darin, die primäre Alkoholfunktion wieder freizusetzen. Dies gelang unter sauren Bedingungen bei $0{ }^{\circ} \mathrm{C}$ durch den Einsatz von $p$-TsOH $\cdot \mathrm{H}_{2} \mathrm{O}$ in $\mathrm{MeOH}$ problemlos. Der freie Alkohol 102 konnte in 
ausgezeichneter Ausbeute von 97\% isoliert werden (Abbildung 33). ${ }^{18 b}$ Auf dieser Stufe wurde das Minderdiastereomer durch präparative HPLC an chiraler stationärer Phase abgetrennt, so dass die Verbindung 102 nun enantio- und diastereomerenrein vorlag. Die Bestimmung der relativen Konfiguration gelang durch Vergleich der Drehwerte der beiden Diastereomere unter Korrelation einer ähnlichen Verbindung, die von C. Stadler ${ }^{18 b}$ aus dem Arbeitskreis Tietze synthetisiert wurde. Darüber hinaus konnte durch zusätzliche NOESY-Experimente zweifelsfrei die cis-Verknüpfung der Ringe $B$ und $\mathrm{C}$ belegt werden. Auch die trans-Ständigkeit der Protonen 3'-H und 3a'-H konnte auf diese Weise verifiziert werden. Abschließend wurde die freie Alkoholfunktion mit Hilfe von Dess-Martin-Periodinan (DMP) in guter Ausbeute von 89\% oxidiert und somit die Synthese des Tricyclus 60 vollendet (Abbildung 33).

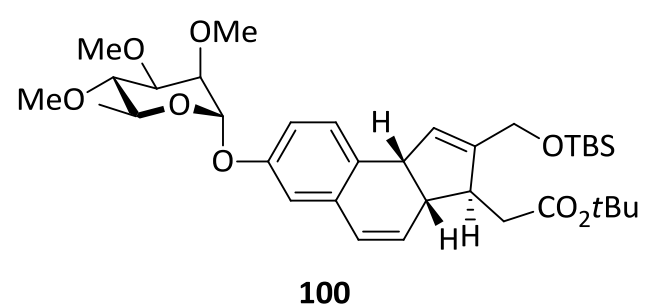

+Diastereomer

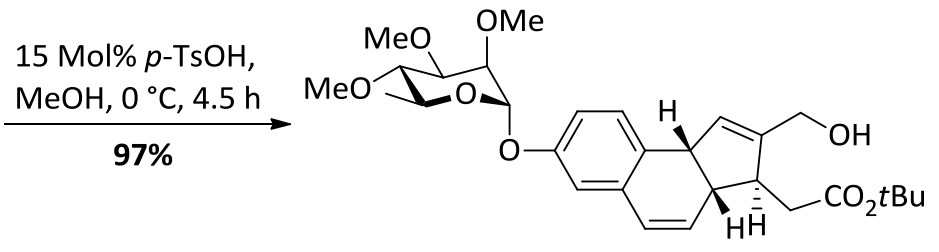

102

+Diastereomer

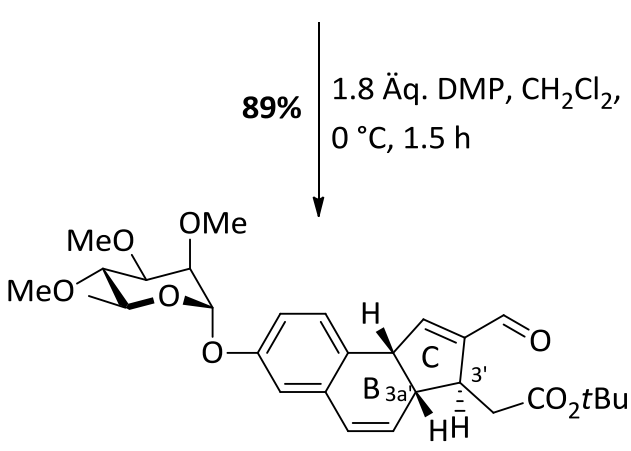

60

Abbildung 33: $\quad$ Synthese des Aldehyds 60.

\subsection{Diskussion ausgewählter spektroskopischer Daten der Verbindung 60}

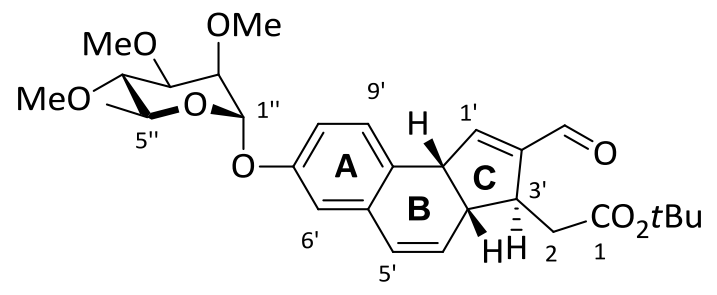

In Abbildung 34 ist das ${ }^{1} \mathrm{H}-\mathrm{NMR}$-Spektrum $\left(600 \mathrm{MHz}, \mathrm{CDCl}_{3}\right.$ ) der Verbindung 60 mit der entsprechenden Zuordnung der Signale dargestellt. Im Hochfeldbereich des 
Spektrums findet man zunächst ein Dublett bei $\delta=1.23 \mathrm{ppm}$ mit einer Kopplungskonstanten von $J=6.2 \mathrm{~Hz}$. Dieses Signal lässt sich den drei Protonen an C-6" zuordnen. Das Singulett mit einer Intensität von 9 bei $\delta=1.44 \mathrm{ppm}$ ist auf die Protonen des tert-Butylesters zurückzuführen. Die beiden diastereotopen Protonen 2- $\mathrm{H}_{\mathrm{A}}$ und $2-\mathrm{H}_{\mathrm{B}}$ resonieren bei $\delta=2.27$ bzw. $2.77 \mathrm{ppm}$ jeweils als Dublett vom Dublett. Sie besitzen eine gemeinsame geminale Kopplungskonstante von $J=15.9 \mathrm{~Hz}$, aber unterschiedliche vicinale Kopplungskonstanten von $J=10.7 \mathrm{~Hz}\left(2-\mathrm{H}_{\mathrm{A}}\right)$ und $J=3.6 \mathrm{~Hz}$ $\left(2-\mathrm{H}_{\mathrm{B}}\right)$. Die Resonanzfrequenz des 4"-H-Protons beträgt $\delta=3.17 \mathrm{ppm}$.

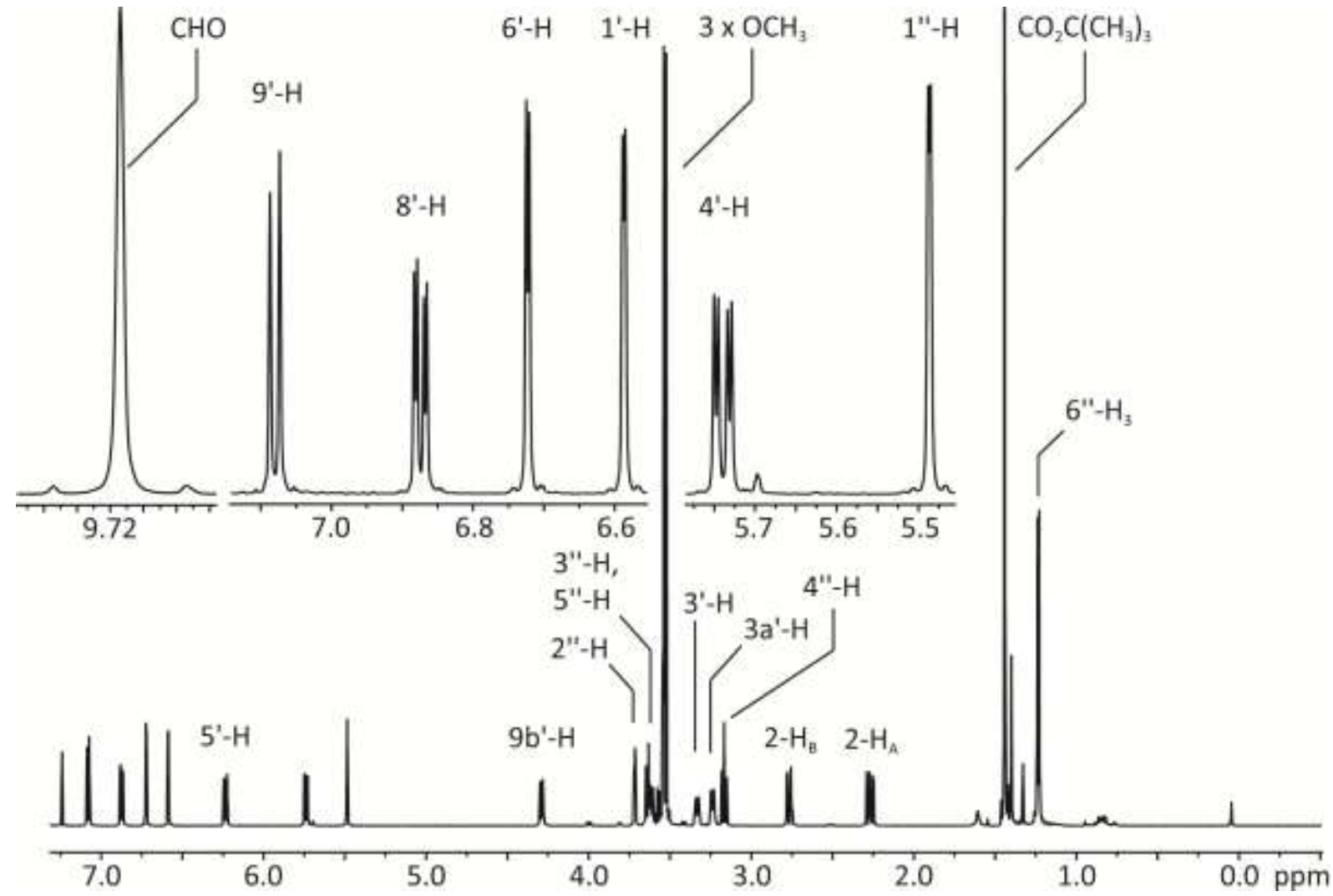

Abbildung 34: $\quad{ }^{1} \mathrm{H}-\mathrm{NMR}-S p e k t r u m\left(600 \mathrm{MHz}^{\left.-\mathrm{CDCl}_{3}\right)}\right.$ der Verbindung 60.

Hierfür wird ein Triplett mit $J=9.4 \mathrm{~Hz}$ gefunden. Dies lässt sich dadurch erklären, dass die beiden axial-axial-Kopplungen mit den benachbarten Protonen identisch sind. Die beiden allylischen Protonen $3 \mathrm{a}^{\prime}-\mathrm{H}$ und 3'-H resonieren jeweils als zentriertes Multiplett bei $\delta=3.24 \mathrm{ppm}$ und $\delta=3.33 \mathrm{ppm}$. Durch NOESY-Experimente konnte zudem die trans-Ständigkeit dieser beiden Protonen sowie die cis-Ständigkeit von 3a'-H und 9b'-H bestätigt werden. Die drei Methoxygruppen der Zuckereinheit weisen sehr ähnliche Resonanzfrequenzen auf und sind als drei Singuletts zwischen $\delta=3.52$ und $3.54 \mathrm{ppm}$ zu finden. Das Multiplett im Bereich von $\delta=3.59-3.64 \mathrm{ppm}$ ist dem Proton 
an C-5" zuzuordnen. Das 3"-H hingegen resoniert mit einer axial-axial-Kopplung von $J=9.4 \mathrm{~Hz}$ und einer äquatorial-axial-Kopplung von $J=3.3 \mathrm{~Hz}$ bei $\delta=3.64$ ppm in Form eines sauberen Dubletts vom Dublett. Das benachbarte Proton an C-2" weist die charakteristischen Kopplungskonstanten einer äquatorial-axial-Kopplung $(J=3.1 \mathrm{~Hz})$ sowie einer äquatorial-äquatorial-Kopplung $(J=2.1 \mathrm{~Hz})$ auf und hat eine Resonanzfrequenz von $\delta=3.72 \mathrm{ppm}$. Das zum aromatischen System in benzylischer Position stehende Proton an C-9b' resoniert als zentriertes Multiplett bei $\delta=4.29 \mathrm{ppm}$. Das Signal für das Proton am anomeren Zentrum der Zuckereinheit 1 "- $H$ ist bei $\delta=5.49 \mathrm{ppm}$ zu finden. Die starke Verschiebung in den Tieffeldbereich und die Tatsache, dass es sich bei dem Wert für die Kopplungskonstante $(J=1.8 \mathrm{~Hz})$ um einen sehr kleinen Wert handelt, der typisch für äquatorial-äquatorial-Kopplungen ist, lässt darauf schließen, dass es sich um ein $\alpha$-Glykosid handeln muss. Die beiden olefinischen Protonen des B-Rings 4'-H bzw. 5'-H zeigen sich im Spektrum jeweils in Form eines Dubletts vom Dublett und weisen eine für Doppelbindungsprotonen charakteristische chemische Verschiebung von $\delta=5.74\left(4^{\prime}-\mathrm{H}\right)$ bzw. 6.23 ppm (5'-H) auf. 5'-H steht dabei unter dem Einfluss des aromatischen A-Rings und ist deshalb weiter zum Tieffeldbereich verschoben. Das dritte olefinische Proton dieser Verbindung an C-1' erscheint als zentriertes Multiplett und steht in Konjugation zur Aldehydfunktion. Es besitzt daher mit $\delta=6.59 \mathrm{ppm}$ die größte chemische Verschiebung dieser Protonenart. Im aromatischen Bereich des Protonenspektrums lässt sich das markante Kopplungsmuster eines 1,2,4-trisubstituierten Aromaten finden. Das Proton an C $-6^{\prime}$ resoniert bei $\delta=6.72 \mathrm{ppm}$ als Dublett mit einer charakteristischen meta-Kopplungskonstanten von $J=2.6 \mathrm{~Hz}$. Das dazu in meta-Position stehende $8^{\prime}-\mathrm{H}$ wird bei $\delta=6.87 \mathrm{ppm}$ als Dublett vom Dublett gefunden. Dies ist darauf zurückzuführen, dass es neben der meta-Kopplung $(J=2.6 \mathrm{~Hz})$ auch eine ortho-Kopplung $(J=8.3 \mathrm{~Hz})$ mit 9'-H aufweist. Eben dieses Proton an C-9' besitzt eine Resonanzfrequenz von $\delta=7.08 \mathrm{ppm}$ und das Vorliegen eines Dubletts mit $J=8.3 \mathrm{~Hz}$ bestätigt die ortho-Ständigkeit zu 8'-H. Der Signalsatz des Protonenspektrums wird im Tieffeldbereich komplettiert durch das Singulett des Aldehydprotons bei $\delta=9.72 \mathrm{ppm}$. 
Charakteristische Signale des ${ }^{13}$ C-NMR-Spektrums (Abbildung 35) des Tricyclus 60 lassen sich bei $\delta=17.9 \mathrm{ppm}$ (C-6") und für die tert-Butylgruppierung bei $\delta=28.2 \mathrm{ppm}$ $\left(\mathrm{CO}_{2} \mathrm{C}\left(\mathrm{CH}_{3}\right)_{3}\right)$ bzw. $\delta=80.7 \mathrm{ppm}\left(\mathrm{CO}_{2} \underline{\mathrm{C}}\left(\mathrm{CH}_{3}\right)_{3}\right)$ finden. Darüber hinaus resoniert das C-2-Atom im Hochfeldbereich bei $\delta=38.4$ ppm. Die drei tertiären Kohlenstoffatome C-3a', C-9b' und C-3' weisen eine sehr ähnliche chemische Verschiebung auf und sind im Bereich zwischen $\delta=44.1$ und 47.4 ppm zu finden. Die drei Methoxysubstituenten der Zuckereinheit resonieren bei $\delta=57.9,59.2$ sowie $60.9 \mathrm{ppm}$. Das Kohlenstoffatom C-1" am anomeren Zentrum ist mit $\delta=95.2 \mathrm{ppm}$ das am weitesten in den Tieffeldbereich verschobene Ringkohlenstoffatom des Zuckers.

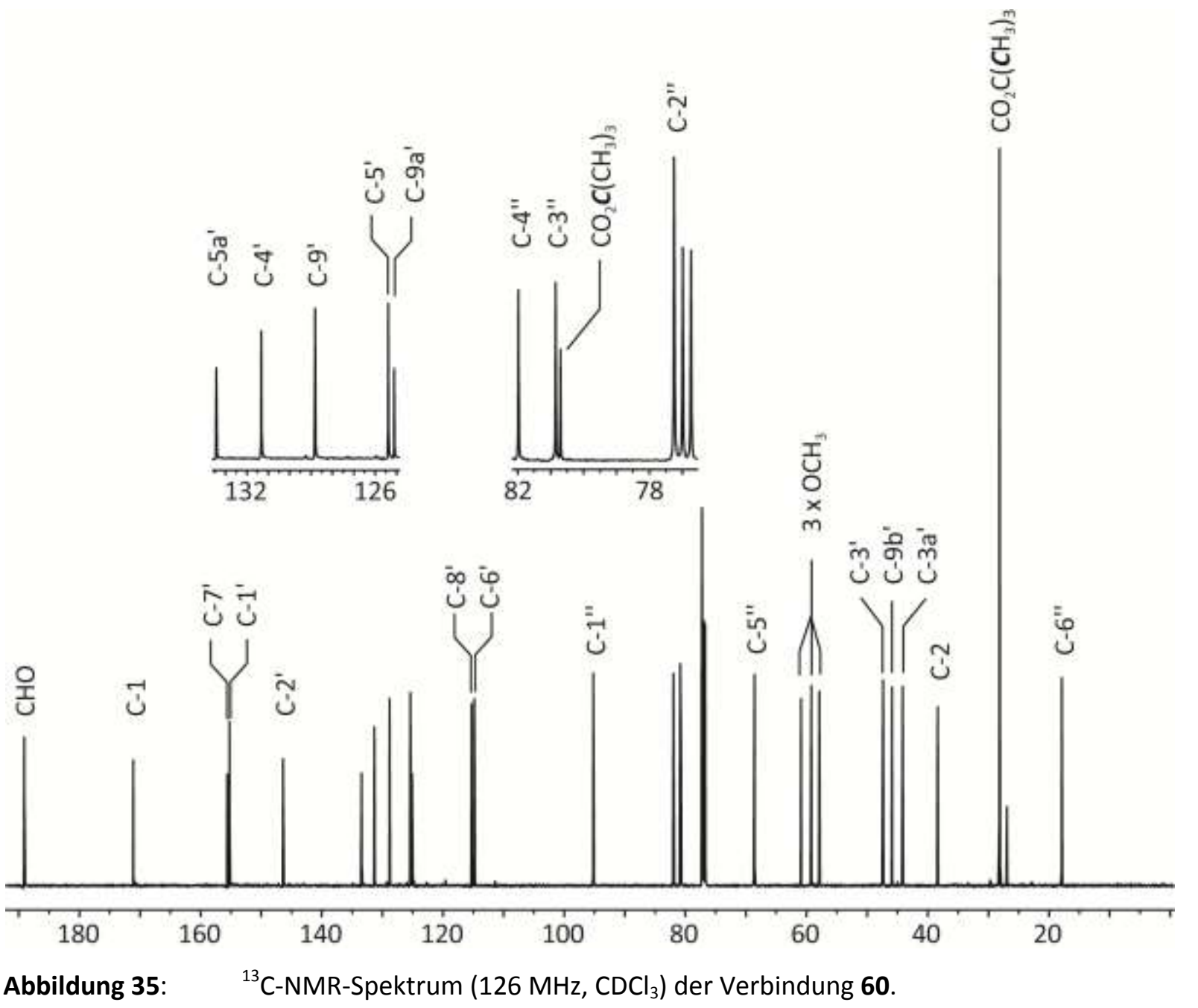

Die übrigen resonieren im Bereich von $\delta=68.6$ und $82.0 \mathrm{ppm}$. Im olefinischen und aromatischen Bereich des Kohlenstoffspektrums finden sich wie erwartet zehn Signale für die sechs Kohlenstoffe des A-Rings und die vier Doppelbindungskohlenstoffe. Besonders hervorzuheben ist hier die starke Entschirmung des C-2' und 
vor allem des $\mathrm{C}^{-1}$ ', die aufgrund der Konjugation zur Aldehydfunktion zustandekommt. Am weitesten in den Tieffeldbereich verschoben sind die Carbonyl-C-Atome der Esterfunktion und des Aldehyds, die bei $\delta=171.1 \mathrm{ppm}(\mathrm{C}-1) \mathrm{bzw}$. $\delta=189.1 \mathrm{ppm}$ $(\mathrm{CHO})$ resonieren.

Das ESI-Massenspektrum zeigt zwei signifikante Peaks mit $m / z=1051.5$ und 537.2. Diese lassen sich der Verbindung 60 als $[2 \mathrm{M}+\mathrm{Na}]^{+}$bzw. $[\mathrm{M}+\mathrm{Na}]^{+}$zuordnen. Darüber hinaus bestätigt die ESI-Hochauflösung die chemische Zusammensetzung mit der Summenformel $\mathrm{C}_{29} \mathrm{H}_{38} \mathrm{O}_{8}$. 


\section{Synthese der C-9-Fragmente $\alpha-103$ und $\beta-103$}

\subsection{Synthese der C-6-Bausteine $\alpha-115$ und $\beta-115$}

Für die $\beta$-selektive Einführung der D-Forosamineinheit wurde das literaturbekannte Hexenol 107 verwendet. $^{63}$ Die von Renaud et al. beschriebene Synthesesequenz zu Verbindung 107 führt dabei auf schnelle und elegante Weise hochdiastereoselektiv zu der gewünschten syn-Anordnung der beiden Hydroxyfunktionalitäten.

1.2 Äq. 108, $8 \mathrm{Mol} \% \mathrm{TfOH}$,

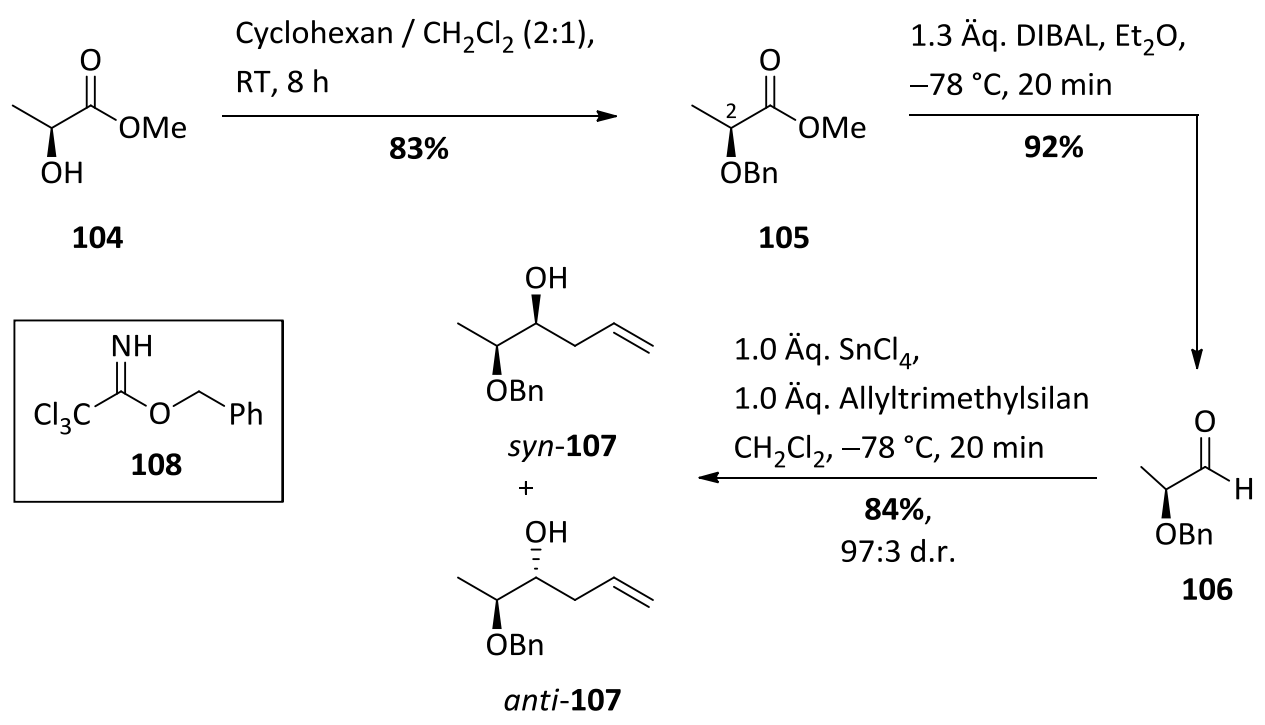

Abbildung 36: $\quad$ Synthese des syn-Aglykons 107.

Als Ausgangsverbindung diente das sehr günstig erhältliche (S)-Methyllactat (104), das mit 94\% ee geliefert wurde. Den ersten Schritt der dreistufigen Synthesesequenz stellte die Schützung des sekundären Alkohols dar. Hierfür wurde eine literaturbekannte Methode ${ }^{64}$ angewendet, die unter sehr milden Bedingungen und unter Verwendung von Trichloracetimidat 108 als Benzylierungsmittel Säure-katalysiert die Benzylschutzgruppe einführt. Dies gelang in guten Ausbeuten, so dass Verbindung 105 in 83\% isoliert werden konnte (Abbildung 36). Eine Benzylschützung von Verbindung 104 unter Einsatz von $\mathrm{NaH}$ als Base und Benzylbromid war zwar ebenfalls erfolgreich, führte aber in hohem Maße zur Racemisierung des stereogenen Zentrums. Darüber hinaus wurde zuvor auch eine Schützung als TBS-Ether getestet, was jedoch im späteren Verlauf der Synthese keinen selektiven Aufbau des zweiten stereogenen Zentrums ermöglichte. 
Das benzylgeschützte Lactat 105 sollte nun in einen Aldehyd überführt werden. Dies war selektiv unter Verwendung des Reduktionsmittels DIBAL bei einer Reaktionsführung bei $-78{ }^{\circ} \mathrm{C}$ möglich. Die sehr guten Ausbeuten für den Aldehyd 106 von bis zu 92\% wurden vor allem dann erreicht, wenn die DIBAL-Lösung aus einem mit Trockeneis gekühlten Tropftrichter zugegeben wurde.

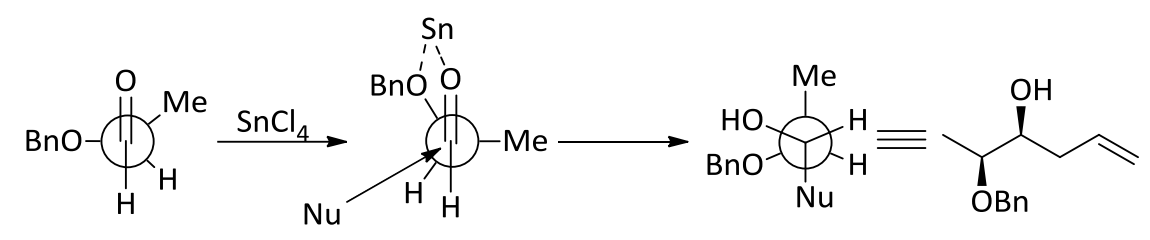

Abbildung 37: Schematische Darstellung der Cram-Chelat-Kontrolle.

Der letzte Schritt der Synthesesequenz war eine Lewis-Säure-vermittelte Allylierung des Aldehyds 106. Diese Reaktion verlief aufgrund von Cram-Chelat-Kontrolle (Abbildung 37) hoch diastereoselektiv, so dass das zweite stereogene Zentrum in einem Verhältnis von syn/anti = 97:3 aufgebaut werden konnte. Das Diastereomerengemisch (84\% Gesamtausbeute) konnte problemlos säulenchromatographisch getrennt und das gewünschte syn-Aglykon 107 mit 81\% Ausbeute erhalten werden. Jedoch kam es auch im Verlauf dieser Synthesesequenz, vermutlich im Reduktionsschritt, zu einer partiellen Racemisierung des Substrats, so dass abschließend nur noch ein Enantiomerenüberschuss von $86 \%$ gefunden wurde. Dies sollte allerdings kein Problem darstellen, da eine Trennung der Enantiomere mittels präparativer HPLC an chiraler stationärer Phase zu einem späteren Zeitpunkt der Synthese möglich sein sollte. Wegen der besseren Übersichtlichkeit wird in der Folge ausschließlich das Hauptenantiomer abgebildet. Eine solche Chelat-Kontrolle wäre mit der TBS-Schutzgruppe nicht möglich gewesen, weshalb dieser Weg nicht weiter verfolgt wurde.

Die anschließende Glykosidierung des Bausteins 107 war Gegenstand der Dissertation $^{65}$ von S. Dietz aus dem Arbeitskreis Tietze. Es wurde explizit ein solch früher Punkt der Synthesesequenz zur Glykosidierung gewählt, da in früheren Arbeiten im Arbeitskreis Tietze die Einführung der D-Forosamineinheit an komplexeren Vorstufen der Spinosynanaloga entweder gar nicht oder nur in moderaten Ausbeuten sowie mit nicht zufriedenstellenden $\beta$-Selektivitäten realisiert werden konnte. 


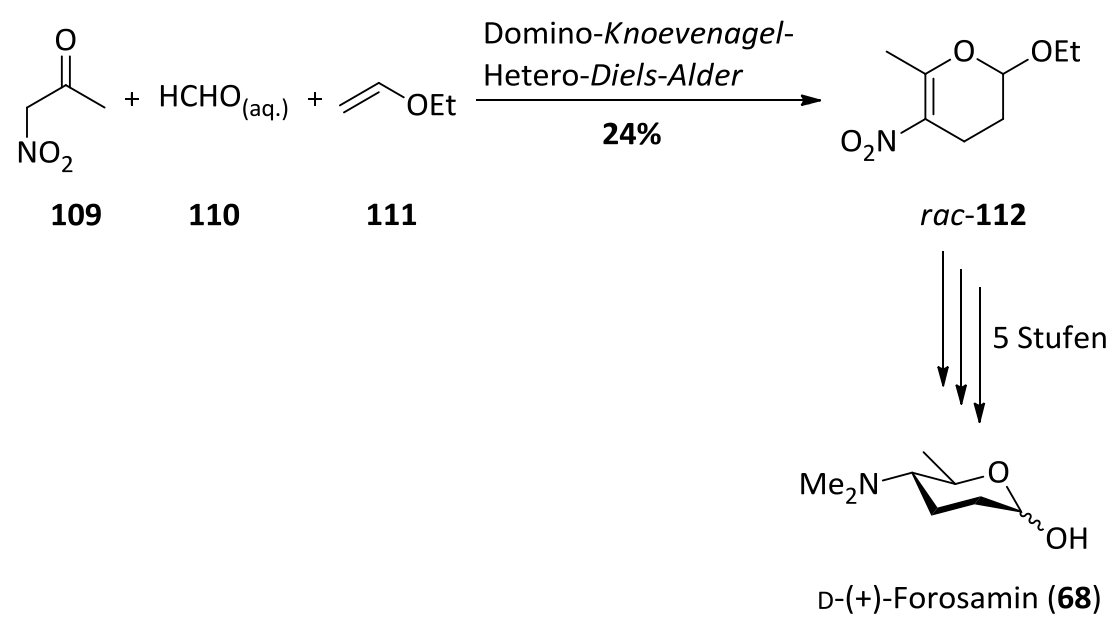

Abbildung 38: Schematische Darstellung der Synthese von enantiomerenreinem D-Forosamin nach S. Dietz.

S. Dietz gelang es, eine neue effiziente Synthese von enantiomerenreinem D-Forosamin (68) zu etablieren. Sie konnte ausgehend von Nitroaceton (109), Formaldehyd (110) und Ethylvinylether (111) in nur sechs Stufen den hochgradig desoxygenierten Zucker herstellen (Abbildung 38). Der Schlüsselschritt der Synthese war eine von N. Böhnke und S. Dietz im Arbeitskreis Tietze entwickelte Domino-KnoevenagelHetero-Diels-Alder-Reaktion. ${ }^{66}$ Darüber hinaus stellte sie im Rahmen ihrer Arbeit eine Reihe geeigneter Forosamindonoren zur Glykosidierung des Hexenols 107 bereit. Als wirkungsvollster Kandidat stellte sich dabei das Trichloracetimidat $\mathbf{1 1 3}$ heraus, das als Gemisch der Anomere $(\alpha / \beta \approx 1: 9)$ synthetisiert wurde. Seine Azid-Funktion an C-4 war ideal dazu geeignet, zu einem beliebigen späteren Zeitpunkt eine Vielzahl an Derivaten bereitzustellen oder auf kurzem Weg die für das Spinosynderivat 58 benötigte Dimethylaminofunktion der D-Forosamineinheit zu installieren.

Die Glykosidierung erfolgte unter Verwendung von $\mathrm{Et}_{3} \mathrm{SiH}$ und elementarem lod bei tiefer Temperatur von $-90^{\circ} \mathrm{C}^{67}$ Es wurde ein 7:5-Gemisch der Anomere $\alpha-114$ und ß-114 in exzellenter Gesamtausbeute von 96\% erhalten (Abbildung 39).

Allerdings konnte nicht die gewünschte Bevorzugung der $\beta$-Verknüpfung, wie sie in den natürlich vorkommenden Spinosynen A (30) und D (31) vorliegt (vergleiche Abbildung 18), erreicht werden. Das Ergebnis war jedoch sowohl bezogen auf die Ausbeute als auch auf die Selektivität besser als die zuvor im Arbeitskreis Tietze erzielten Ergebnisse. 


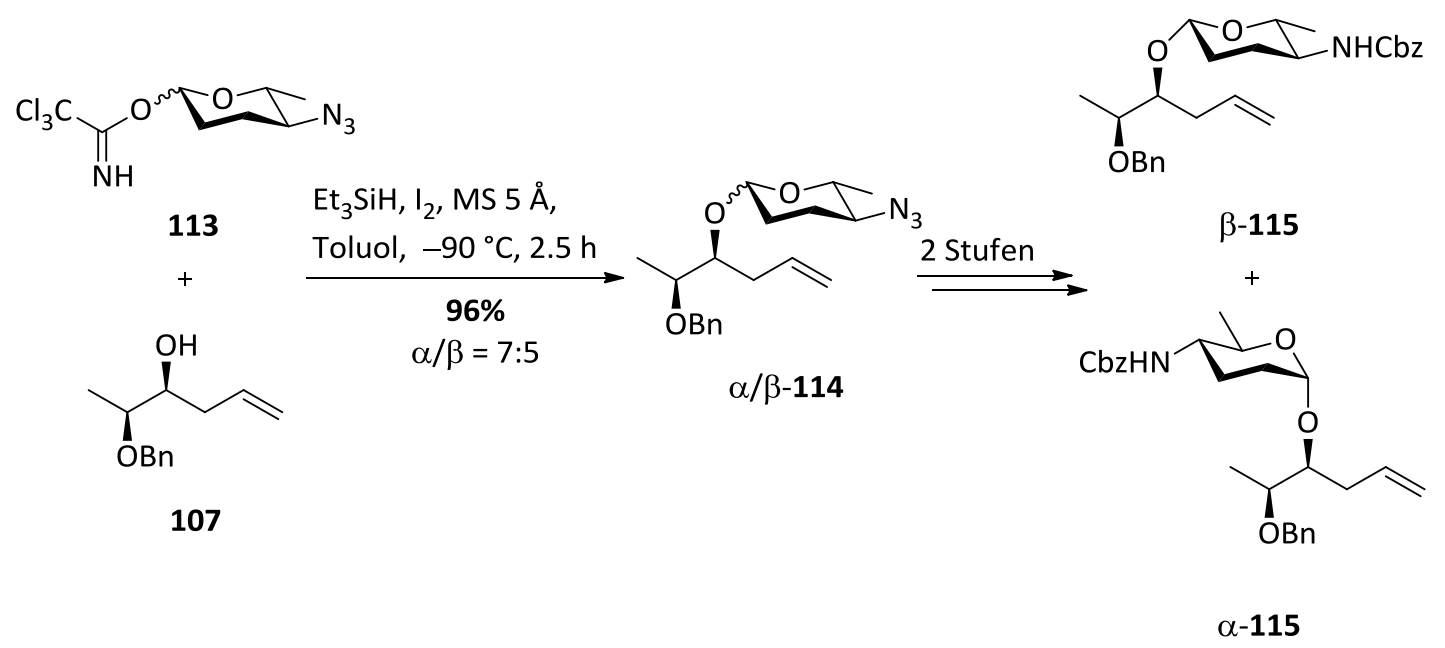

Abbildung 39: $\quad$ Schematische Darstellung der Synthese der Glykoside $\alpha-\mathbf{1 1 5}$ und $\mathbf{\beta - 1 1 5 . ~}$

Darüber hinaus war es möglich, das Anomerengemisch durch säulenchromatographische Reinigung an Kieselgel zu trennen und so die reinen Verbindungen $\mathbf{\alpha - 1 1 4}$ und ß-114 zu erhalten. Wie oben bereits erwähnt, stellte das Azid eine geeignete Ausgangsverbindung dar, um eine Reihe von Derivaten herzustellen. ${ }^{65}$ Als aussichtsreichste Verbindungen für die spätere Synthese stellten sich die Cbz-geschützten Derivate $\alpha-\mathbf{1 1 5}$ und $\beta-\mathbf{1 1 5}$ heraus, die problemlos ausgehend von Verbindung $\mathbf{1 1 4}$ in zwei weiteren Stufen in sehr guten Ausbeuten hergestellt werden konnten (Abbildung 39).

\subsection{Synthese des enantiomerenreinen C-5-Bausteins $\mathbf{1 1 8}$}

Die Kettenverlängerung der oben beschriebenen C-6-Bausteine sollte mittels der in der Synthesechemie weit verbreiteten Metathese-Reaktion geschehen. ${ }^{68}$ Hierfür wurde ein C-5-Baustein mit endständiger Doppelbindung benötigt, der zusätzlich ein (S)-konfiguriertes stereogenes Zentrum an C-3 trägt. Für die spätere Synthese wäre 1-Penten-3-ol (116) ideal geeignet. Dieser Baustein war jedoch nur als racemisches Gemisch käuflich erhältlich. Eine asymmetrische Synthese dieses kleinen Bausteins wurde zwar angestrebt, aber nach wenigen nicht erfolgreichen Versuchen wieder aufgegeben. Im Verhältnis zur Größe des Bausteins wäre dieses Vorhaben zu aufwändig und zeitintensiv gewesen. Als Alternative blieb nun noch eine kinetische Racematspaltung. Dabei wird ausgenutzt, dass eines der beiden Enantiomere in einer Reaktion, z. B. unter Verwendung eines Katalysators oder eines Enzyms, schneller 
reagiert als das andere. Idealerweise sollte bei $50 \%$ Umsatz nur das Produkt des schneller reagierenden Enantiomers gebildet werden, so dass es zu einer Anreicherung des nicht umgesetzten Enantiomers sowie des zum Produkt reagierten anderen Enantiomers kommt. Der große Nachteil dieses Verfahrens ist offensichtlich, da maximal eine theoretische Ausbeute von $50 \%$ erreicht werden kann. Eine in der Literatur weit verbreitete Reaktion dieser Art ist die von $K$. B. Sharpless entwickelte und nach ihm benannte Sharpless-Epoxidierung. ${ }^{69}$ Bei dieser Reaktion wird unter Verwendung eines chiralen Titan-Tartrat-Komplexes (D- oder L-Tartrat) gezielt nur eines der beiden Enantiomere eines Allylalkohols epoxidiert. Da es sich bei 1-Penten-3-ol (116) ebenfalls um einen Allylalkohol handelt, schien die SharplessEpoxidierung die ideale Variante zur Herstellung des enantiomerenreinen Bausteins zu sein. Allerdings waren die Ergebnisse unter verschiedenen Bedingungen nicht zufriedenstellend. Auf diese Weise konnte ein maximaler Enantiomerenüberschuss von $51 \%$ für das gewünschte (S)-Enantiomer erreicht werden. Hinzu kam, dass sich das kleine Molekül als sehr flüchtig erwies und damit schlecht zu handhaben war.

Eine andere Art der kinetischen Racematspaltung macht sich die Eigenschaften von Enzymen zunutze. Bei dieser enzymatischen Racematspaltung werden Hydrolasen eingesetzt. Die häufigsten Reaktionstypen sind dabei Veresterungen und Esterspaltungen, bei denen die Enzyme eines der beiden Enantiomere bevorzugt umsetzen. Eine weitverbreitete Methode ist z. B. die enzymatische Übertragung eines Acetats auf eine freie Hydroxyfunktion unter Verwendung von Vinylacetat als Acetatquelle. Diese Reaktion sollte sich also auch für die Synthese des benötigten enantiomerenreinen Bausteins 118 eignen. Dazu wurden verschiedene Enzyme und Bedingungen getestet. Wie bereits weiter oben beschrieben, war das Pentenol 116 leicht flüchtig, was die Isolierung erschwerte. Die Lösung dafür war die direkte Überführung des Produktes der Racematspaltung in das Dinitrobenzoat 118 (siehe Abbildung zu Tabelle 5). Dies hatte den Vorteil, dass es sich bei dem Dinitrobenzoat 118 auf der einen Seite um einen kristallinen Feststoff handelt und auf der anderen Seite um eine chromophore Einheit, die eine Trennung der Enantiomere mittels 
präparativer HPLC ermöglicht. Die Ergebnisse dieser Umsetzung sind in Tabelle 5 dargestellt.

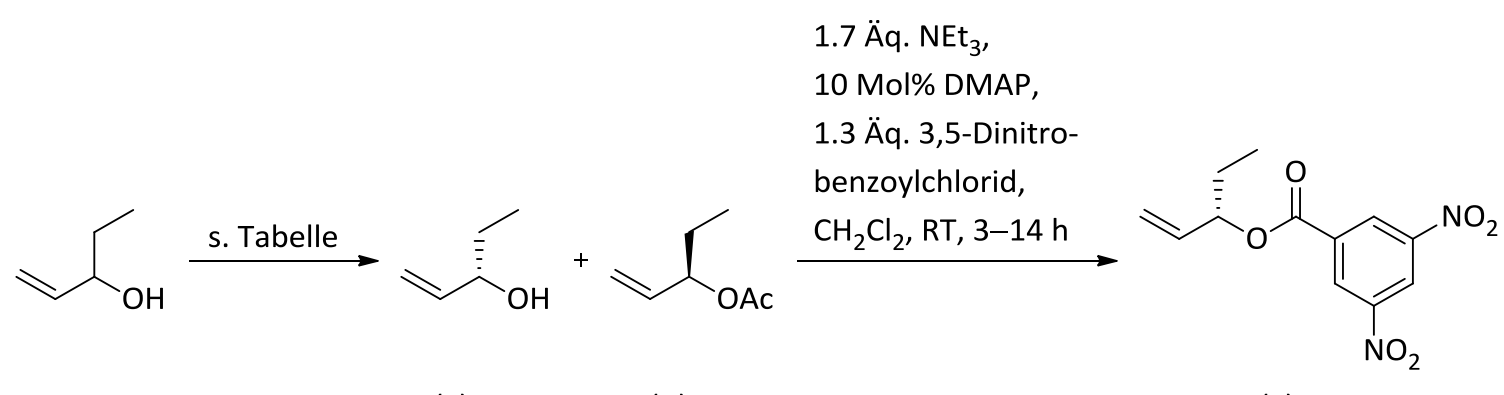

rac-116

(S)-116

$(R)-117$

(S)-118

\begin{tabular}{|c|c|c|c|}
\hline \# & Enzym & Bedingungen & Ausbeute $^{[\mathrm{a}]}$ \\
\hline 1 & Pancreatin & $\begin{array}{c}0.7 \ddot{A q} . \mathrm{NEt}_{3}, 2.1 \text { Äq. Vinylacetat, } \\
\text { THF, RT, } 6 \text { d }\end{array}$ & $\begin{array}{c}22 \% \\
(75 \% \text { ee })\end{array}$ \\
\hline 2 & Amano Lipase AK & $\begin{array}{c}2.1 \text { Äq. Vinylacetat, MS } 4 \AA \text {, } \\
n \text {-Hexan, RT, } 5.5 \mathrm{~h}\end{array}$ & $\begin{array}{l}13 \% \\
(66 \% \text { ee })\end{array}$ \\
\hline 3 & Amano Lipase AK & $\begin{array}{c}2.0 \text { Äq. Vinylacetat, MS } 4 \AA \text {, } \\
\text { n-Hexan, RT, } 6 \mathrm{~h}\end{array}$ & $\begin{array}{c}25 \% \\
(75 \% \text { ee })\end{array}$ \\
\hline 4 & Amano Lipase AK & $\begin{array}{c}2.0 \text { Äq. Vinylacetat, MS } 4 \AA \text {, } \\
\text { n-Hexan, RT, } 7 \mathrm{~h}\end{array}$ & $\begin{array}{c}23 \% \\
(81 \% \text { ee })\end{array}$ \\
\hline 5 & Lipase B (Novozym 435) & $\begin{array}{c}0.8 \text { Äq. Vinylacetat, MS } 4 \text { Á, Toluol, } \\
\text { RT, } 7 \mathrm{~h}\end{array}$ & $\begin{array}{c}46 \% \\
(16 \% \text { ee })^{[\mathrm{b}]}\end{array}$ \\
\hline
\end{tabular}

[a] Ausbeute und ee-Wert als Dinitrobenzoat 118, Bestimmung des ee-Wertes via HPLC; [b] (R)-Enantiomer

Tabelle 5: Enzymatische Racematspaltung von rac-116 und anschließende Umsetzung zum Dinitrobenzoat 118.

Wie aus Tabelle 5 ersichtlich wird, lieferte das Enzym Amano Lipase $\mathrm{AK}^{70}$ aus Pseudomonas fluorescens die besten Ergebnisse. Die anschließende Trennung der Enantiomere mittels präparativer HPLC an chiraler stationärer Phase lieferte enantiomerenreines (S)-118 mit >99\% ee. Es wurde auch versucht, die Enantiomerenreinheit durch Umkristallisation zu erhöhen. Dies war jedoch nicht effektiv genug und hätte mehrere Umkristallisationsschritte benötigt. Ebenfalls zufriedenstellend waren die Ergebnisse für die Enantiomerenüberschüsse unter Verwendung von Pancreatin ${ }^{71}$ aus der Bauchspeicheldrüse von Hausschweinen. Allerdings war die lange Reaktionszeit von $6 \mathrm{~d}$ 
nicht akzeptabel. Die Verwendung von Lipase $\mathrm{B}^{18 \mathrm{~b}}$ aus Candida antartica, kommerziell erhältlich unter dem Namen Novozym 435, führte zu dem nicht gewünschten Enantiomer $(R)-118$.

\subsection{Synthese der Glykoside $\alpha-103$ und $\beta-103$}

Der nächste Schritt des Syntheseplans sah die Zusammenführung des C-6Bausteins 115 und des enantiomerenreinen Dinitrobenzoats 118 vor. Wie bereits zuvor erwähnt, sollte hierfür eine Olefin-Metathese verwendet werden. Ohne größere Optimierungen wurden schnell geeignete Bedingungen gefunden. ${ }^{72}$ Unter Verwendung des Grubbs-Katalysators 2. Generation konnten die beiden Bausteine in für Kreuzmetathesen guten Ausbeuten von 74\% für das $\alpha$-Glykosid-119 und 70\% für das $\beta$-Glykosid-119 gekuppelt werden. Die Metathese-Addukte wurden jeweils als das Gemisch der Doppelbindungsisomere $(E / Z \approx 7: 1)$ isoliert (Abbildung 40). Die Selektivität dieser Reaktion spielte aber keine Rolle, da die Doppelbindung im späteren Verlauf der Synthese hydriert werden musste.

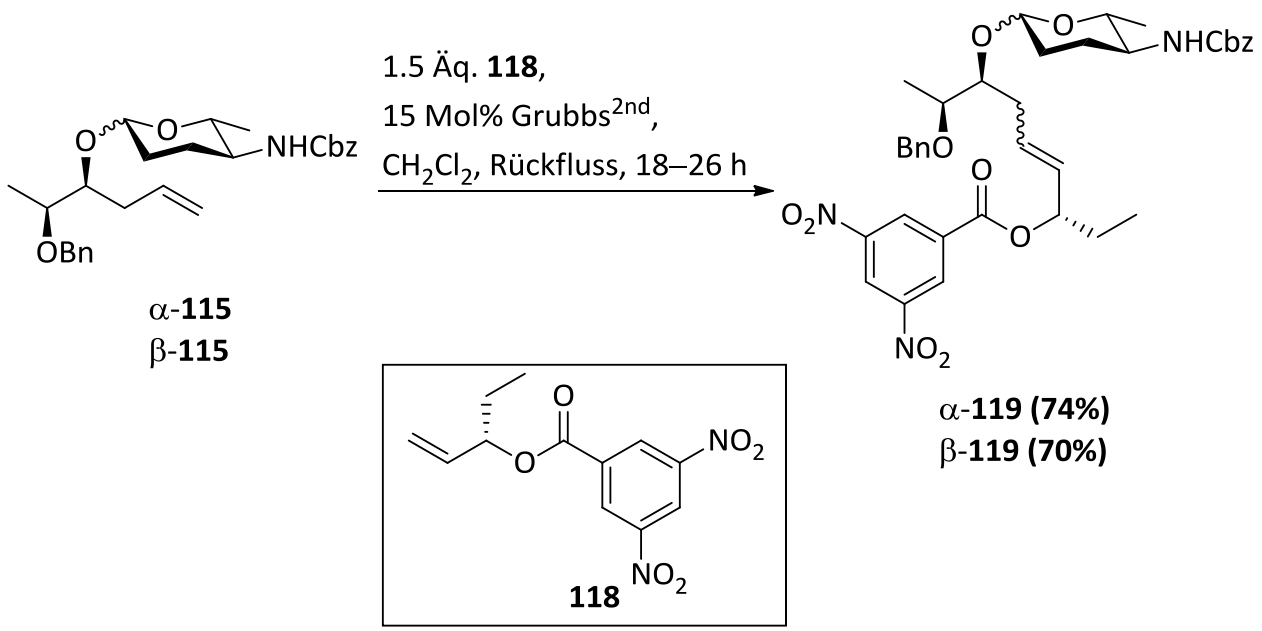

Abbildung 40: $\quad$ Olefin-Metathese von $\alpha-115$ bzw. $\beta-115$ mit 118.

Nun musste die für die Reinigung des C-5-Bausteins benötigte Dinitrobenzoatgruppe wieder entfernt werden, um sie durch eine flexiblere Schutzgruppe zu ersetzen, die eine größere Vielfalt an Reaktionsbedingungen toleriert. Die Spaltung der Esterfunktion erfolgte unter basischen Bedingungen. Die Verwendung von $\mathrm{K}_{2} \mathrm{CO}_{3}$ in einem Methanol / THF-Gemisch lieferte in beiden Fällen den freien Alkohol 120 in guten Ausbeuten (Abbildung 41). 
Als neue Schutzgruppe sollte die TBS-Gruppe dienen. Die Schützung der sekundären Alkoholfunktion war in exzellenten Ausbeuten möglich. Dazu wurde auf ein Standardprotokoll zurückgegriffen, bei dem TBSOTf als Silylquelle und 2,6-Lutidin als Base verwendet wird. Eine Reaktionsführung bei $-10^{\circ} \mathrm{C}$ lieferte die entsprechend geschützten Glykoside in 94\% ( $\alpha-121)$ und 93\% ( $\beta-121)$.<smiles>CC[C@H](/C=C/C[C@@H](O[C@H]1CCC2CC1O2)[C@H](C)OCc1ccccc1)OC(=O)c1cc([N+](=O)[O-])cc([N+](=O)[O-])c1</smiles>

$\beta-119$

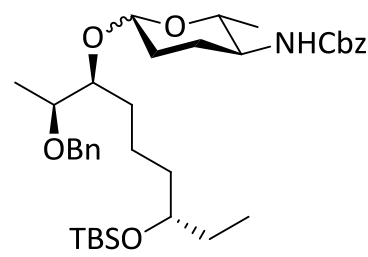

$\alpha-122(88 \%)$

$\beta-122$ (74\%)

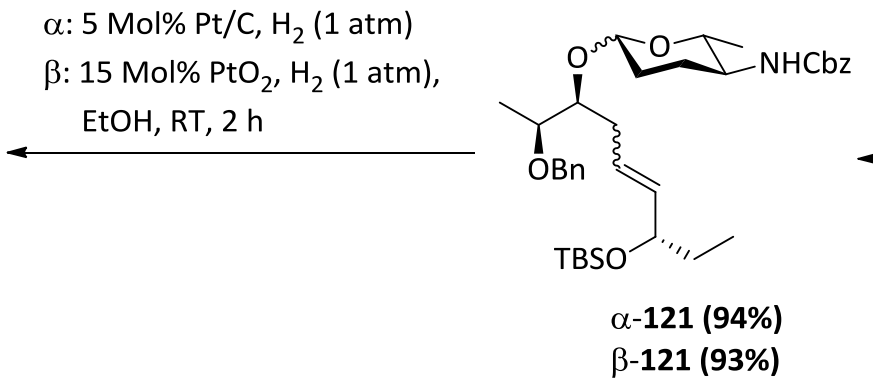

2.0 Äq. 2,6-Lutidin, 1.5 Äq. TBSOTf, $\mathrm{CH}_{2} \mathrm{Cl}_{2}$ $-10^{\circ} \mathrm{C}, 30 \mathrm{~min}$
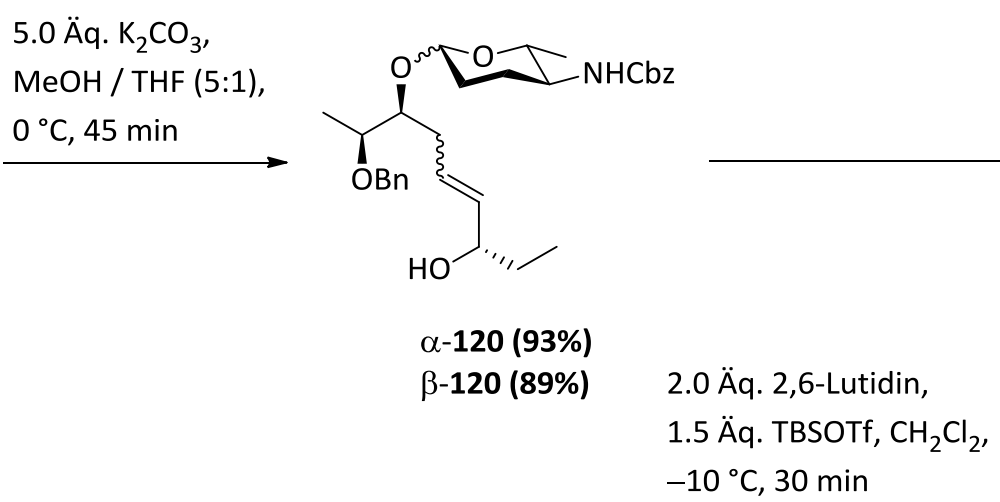

Abbildung 41: $\quad$ Synthese der Glykoside $\alpha-\mathbf{1 2 2}$ und $\beta-\mathbf{1 2 2}$.

An diesem Punkt der Synthese sollte die Hydrierung der Doppelbindung geschehen. Dabei war es von großer Wichtigkeit, eine Hydrierungsmethode zu finden, die selektiv die Doppelbindung in Gegenwart der Cbz- und Benzylschutzgruppe reduziert, denn die beiden Schutzgruppen wurden noch als chromophore Einheiten im Molekül benötigt, um eine spätere Reinigung mittels HPLC zu ermöglichen. Hydrierungsstudien an anderen Derivaten hatten gezeigt, dass das Vorhandensein beider Schutzgruppen essentiell für eine ausreichende UV-Aktivität war. Daher kam eine Hydrierung mit Palladium nicht in Frage, weil es zu einer hydrogenolytischen Spaltung der beiden Schutzgruppen kommen würde.

Platin bzw. Platinsalze hingegen sind dafür bekannt, nur unter speziellen, drastischeren Bedingungen die oben genannten Schutzgruppen zu spalten. Es war interessant zu beobachten, dass die beiden Glykoside $\alpha-\mathbf{1 2 1}$ und $\beta-\mathbf{1 2 1}$ in dieser 
Reaktion unterschiedliche Reaktivitäten aufwiesen (Abbildung 41). Während das $\beta$-Glykosid unter $\mathrm{H}_{2}$-Atmosphäre innerhalb von $2 \mathrm{~h}$ durch $\mathrm{Pt}_{2} \mathrm{O}$ völlig umgesetzt wurde, war dies für das $\alpha$-Glykosid auch nach mehr als $6 \mathrm{~h}$ nicht der Fall. Durch die lange Reaktionszeit traten zudem vermehrt Nebenprodukte auf. Hier musste auf das wesentlich aktivere Katalysatorsystem Pt/C (5\%ig) zurückgegriffen werden. So konnte das aliphatische Glykosid $\alpha-\mathbf{1 2 2}$ nach $2 \mathrm{~h}$ bei RT in guten Ausbeuten bis $\mathbf{8 8 \%}$ isoliert werden. Führte man die Hydrierung des $\beta$-Glykosids unter Verwendung von Pt/C durch, so kam es innerhalb kürzester Zeit zu einer verstärkten Bildung an unerwünschten Nebenprodukten. Den größten Anteil daran hatte eine Spezies bei der die TBS-Schutzgruppe samt Sauerstoffatom aus dem Molekül entfernt und durch ein Wasserstoffatom ersetzt wurde. Aber auch die Verwendung von $\mathrm{Pt}_{2} \mathrm{O}$ war nicht zufriedenstellend. Zwar konnte die gewünschte hydrierte Spezies $\beta$-122 nach 2 h und vollem Umsatz in 74\% erhalten werden, jedoch waren auch hier die Materialverluste durch Bildung von Nebenprodukten signifikant. Es kam zur partiellen Abspaltung der Cbz-Gruppe und völlig unerwartet zu einer Hydrierung des aromatischen Systems der Benzylschutzgruppe zu einem aliphatischen Ring.

Diese Stufe stellte den geeigneten Zeitpunkt dar, an dem die Verbindungen $\mathbf{\alpha - 1 2 2}$ und $\beta$-122 von dem durch partielle Racemisierung zu Beginn der Synthesesequenz erhaltenen Minderenantiomer getrennt werden sollten. Dies gelang ohne Probleme mittels präparativer HPLC an chiraler stationärer Phase (Chiralpak ${ }^{\circledR}$ IA).

Der Syntheseplan sah als nächstes eine simultane Spaltung der Benzyl- und CbzSchutzgruppe sowie eine reduktive Aminierung der dann freigesetzten Aminofunktionalität der Zuckereinheit vor. Unglücklicherweise war die gleichzeitige Spaltung der Schutzgruppen nicht umsetzbar. Als Katalysatorsysteme wurde Pd/C (10\%ig) sowie $\mathrm{Pd}(\mathrm{OH})_{2}$ mit unterschiedlichen Beladungen getestet und die Reaktion bei $\mathrm{H}_{2}$-Drücken zwischen 1 bar und 4 bar durchgeführt. Unter diesen Bedingungen kam es jedoch nur zur Spaltung der Cbz-Schutzgruppe und wenn zusätzlich angestrebt zur reduktiven Aminierung. Eine Spaltung der Benzylschutzgruppe konnte höchstens in Spuren festgestellt werden. Daher wurde beschlossen, diesen Teil der Synthese in zwei Schritte aufzuteilen. Die Spaltung der Cbz-Schutzgruppe und die anschließende 
reduktive Aminierung gelangen bei $\approx 3.8$ bar $\mathrm{H}_{2}$-Druck mit $\mathrm{Pd} / \mathrm{C}$ in einem Lösungsmittelgemisch von $\mathrm{MeOH} / \mathrm{CH}_{2} \mathrm{Cl}_{2}$ / EtOAc (3:1:1) unter Zusatz von Formalin-Lösung. Die gewünschten Verbindungen $\alpha-123$ und $\beta-123$ wurden dabei in Ausbeuten von $82 \%$ bzw. $80 \%$ gebildet (Abbildung 42).

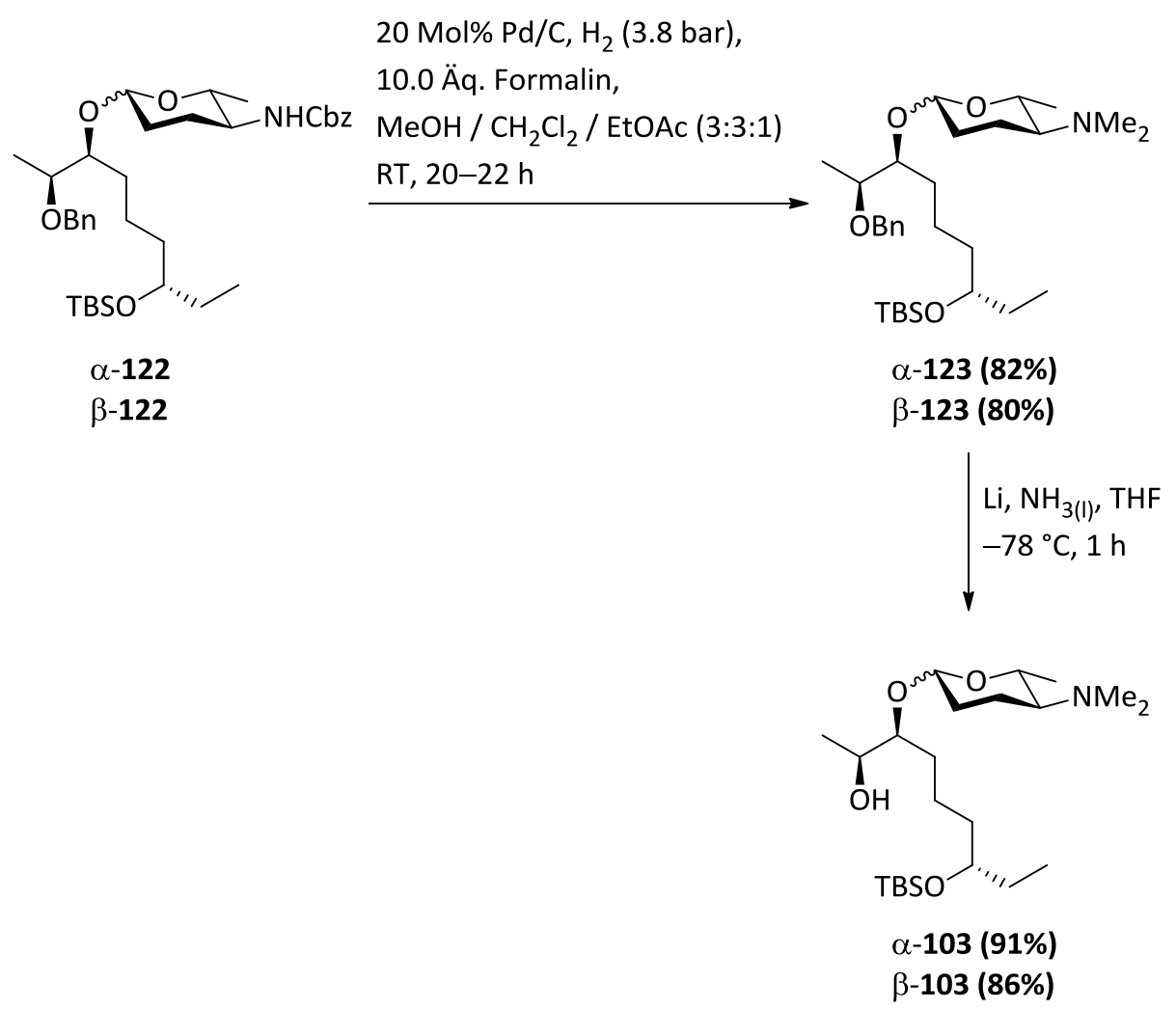

Abbildung 42: Reduktive Aminierung und Birch-Reduktion zur Darstellung von $\alpha-103$ und $\beta-103$.

Die Synthesesequenz sollte nun abschließend durch die Entfernung der Benzylschutzgruppe komplettiert werden. Da die übliche Methode, eine hydrogenolytische Spaltung unter heterogener Katalyse, nicht erfolgreich war, musste auf eine harschere Methode zurückgegriffen werden. In der Literatur ${ }^{55}$ wird dazu die Verwendung der Birch-Reduktion beschrieben. Diese Variante stellte sich in unserem Fall als sehr zuverlässig heraus und lieferte die benötigten Verbindungen mit freier sekundärer Alkoholfunktion in hervorragenden Ausbeuten von 91\% für $\alpha-103$ und 86\% für $\beta-103$ (Abbildung 42). 


\subsection{Diskussion ausgewählter spektroskopischer Daten der Verbindung 及-103}

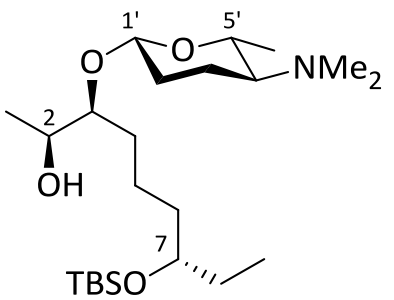

Abbildung 43 zeigt das ${ }^{1} \mathrm{H}-\mathrm{NMR}$-Spektrum $\left(600 \mathrm{MHz}, \mathrm{CDCl}_{3}\right.$ ) von Verbindung $\beta-103$ mit der entsprechenden Zuordnung der Signale. Im Hochfeldbereich des Protonenspektrums lassen sich die Signale für die Protonen der TBS-Schutzgruppe bei $\delta=0.01 \mathrm{ppm}\left(\mathrm{Si}\left(\mathrm{CH}_{3}\right)_{2}\right)$ und $\delta=0.85 \mathrm{ppm}\left(\mathrm{SiC}\left(\mathrm{CH}_{3}\right)_{3}\right)$ jeweils als Singulett beobachten. Weitere charakteristische Signale im Hochfeldbereich sind das Triplett bei $\delta=0.83$ ppm für die drei Protonen an C-9 mit einer Kopplungskonstanten von $J=7.4 \mathrm{~Hz}$ sowie die beiden Dubletts bei $\delta=1.13(J=6.4 \mathrm{~Hz})$ und $1.25 \mathrm{ppm}(J=6.2 \mathrm{~Hz})$. Beide besitzen eine Intensität von 3 und sind somit den Protonen an C-1 bzw. an C-6' zuzuordnen.

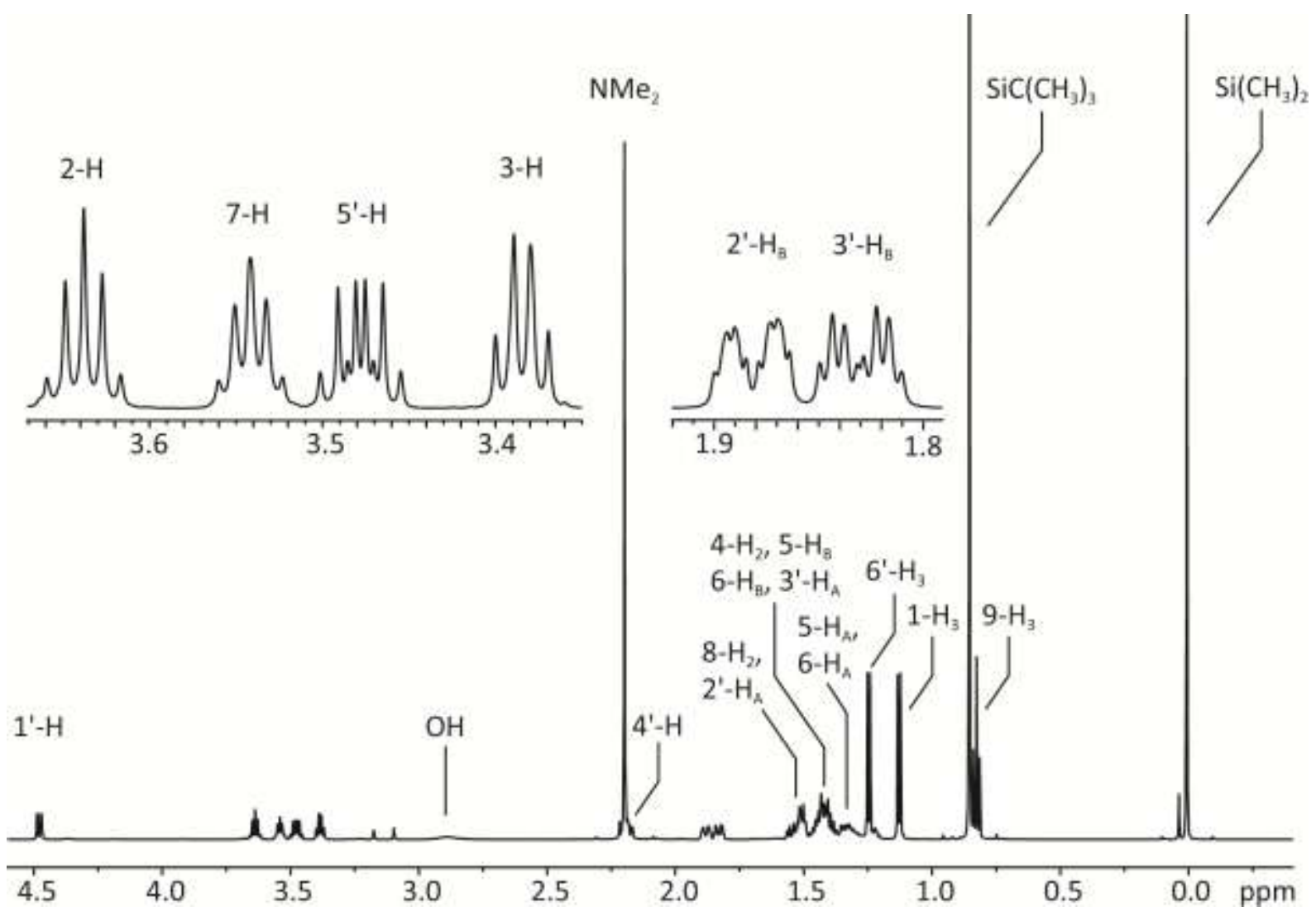

Abbildung 43: $\quad{ }^{1} \mathrm{H}-\mathrm{NMR}-S p e k t r u m\left(600 \mathrm{MHz}, \mathrm{CDCl}_{3}\right)$ der Verbindung $\beta$-103. 
Die diastereotopen Protonen an C-5 und C-6 resonieren jeweils mit leicht unterschiedlicher chemischer Verschiebung. Die Protonen $5-\mathrm{H}_{A}$ und $6-\mathrm{H}_{A}$ sind als Multiplett im Bereich von $\delta=1.29-1.36 \mathrm{ppm} z u$ finden, $5-\mathrm{H}_{B}$ und $6-\mathrm{H}_{B}$ als Multiplett bei $\delta=1.37-1.48 \mathrm{ppm}$. Letzteres Multiplett besitzt eine Intensität von 5 und ist somit das Ergebnis der Signalüberlagerung von drei weiteren Protonen. Darunter zählen zum einen die beiden Protonen an C-4, aber auch eines der beiden diastereotopen Protonen an C-3' (3'- $\left.\mathrm{H}_{\mathrm{A}}\right)$. Allerdings war hier selbst mit Hilfe von COSY-, HSQC- und HMBC-Experimenten keine genauere Beurteilung bzw. Zuordnung der Signale möglich. Das zweite dieser Protonen $\left(3^{\prime}-\mathrm{H}_{B}\right)$ erfährt offensichtlich eine wesentlich stärkere Entschirmung, so dass es bei $\delta=1.83 \mathrm{ppm}$ als Dublett vom Dublett vom Dublett mit Kopplungskonstanten von $J=12.6,7.1$ und $3.6 \mathrm{~Hz}$ resoniert. Bei $\delta=1.48-$ 1.57 ppm liegt ebenfalls ein Multiplett vor, das den beiden Protonen an C-8 und dem $2^{\prime}-\mathrm{H}_{\mathrm{A}}$ zuzuordnen ist. Auch hier ist ein deutlicher Unterschied für die chemische Verschiebung des zweiten dieser diastereotopen Protonen an C-2' zu beobachten. $2^{\prime}-\mathrm{H}_{\mathrm{B}}$ resoniert bei $\delta=1.88 \mathrm{ppm}$ als Dublett vom Dublett vom Dublett mit einer für geminale Kopplungen typischen Kopplungskonstanten von $J=12.6 \mathrm{~Hz}$ und zwei kleineren Kopplungskonstanten $J=5.6$ und $3.4 \mathrm{~Hz}$, die ein Hinweis auf eine äquatorial-axial bzw. äquatorial-äquatorial Kopplung sein könnten. Die sechs Protonen der Dimethylamino-Gruppe an der Zuckereinheit treten im Protonenspektrum als Singulett bei $\delta=2.20$ ppm auf. Ein weiteres Multiplett ist für das $4^{\prime}-\mathrm{H}$ Proton mit einer chemischen Verschiebung von $\delta=2.16-2.22 \mathrm{ppm} z u$ finden. Das Proton der freien Alkoholfunktion besitzt eine Resonanzfrequenz von $\delta=2.89 \mathrm{ppm}$ und ist als breites Singulett zu sehen. Noch weiter zu tiefem Feld verschoben ist das 3-H-Proton, welches bei $\delta=3.38 \mathrm{ppm}$ resoniert. Als sauberes Dublett vom Quartett ist das Proton an $C-5$ ' bei $\delta=3.48$ ppm zu sehen. Die größere Aufspaltung $(J=9.4 \mathrm{~Hz})$ beruht auf einer axial-axial-Kopplung mit dem 4'-H-Proton. Die kleinere Kopplungskonstante weist einen typischen Wert für eine vicinale Kopplung auf $(J=6.2 \mathrm{~Hz})$ und wird durch die drei Protonen der benachbarten Methylgruppe hervorgerufen. Die beiden Quintetts bei $\delta=3.54$ bzw. 3.64 ppm stellen die Signale für das 7-H bzw. das 2-H dar, die jeweils durch charakteristische vicinale Kopplungen $(J=6.2$ bzw. $6.4 \mathrm{~Hz})$ 
aufgespalten werden. Die letzten vier Protonen sind alle aufgrund ihrer unmittelbaren Nachbarschaft zu einem elektronenziehenden Sauerstoffatom wesentlich stärker als die übrigen aliphatischen Protonen entschirmt. Dies gilt auch für das Proton am anomeren Zentrum (1'-H) der Zuckereinheit, das sogar durch zwei Sauerstoffatome entschirmt wird. Es ist mit einer chemischen Verschiebung von $\delta=4.48$ ppm am stärksten in den Tieffeldbereich verschoben und ist dort als Dublett vom Dublett zu sehen. Die große Kopplungskonstante von $J=9.6 \mathrm{~Hz}$ ist typisch für eine axial-axial-Kopplung und die kleinere von $J=2.0 \mathrm{~Hz}$ steht für eine äquatorialaxial-Kopplung. Das Vorhandensein einer axial-axial-Kopplung ist ein zweifelsfreies Zeichen dafür, dass es sich bei Verbindung 103 um ein $\beta$-Glykosid handeln muss, da eine solche Kopplung bei einer $\beta$-Verknüpfung nicht möglich wäre.

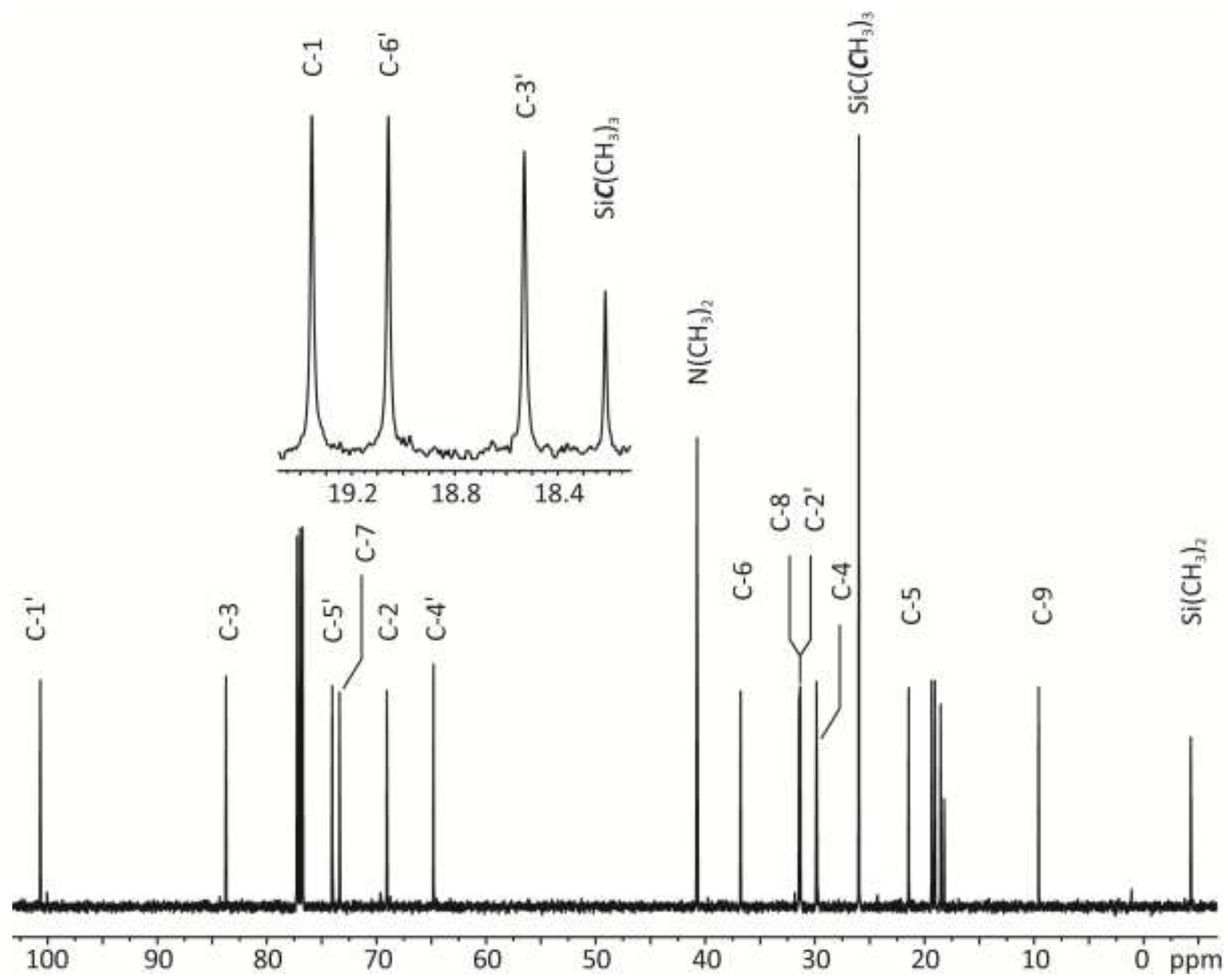

Abbildung 44: $\quad{ }^{13} \mathrm{C}-\mathrm{NMR}$-Spektrum $\left(126 \mathrm{MHz}, \mathrm{CDCl}_{3}\right)$ der Verbindung $\beta$-103.

Das ${ }^{13}$ C-NMR-Spektrum (Abbildung 44) der Verbindung 103 weist bei $\delta=-4.3 \mathrm{ppm}$ zwei Signale für die beiden Methylgruppen am Siliciumatom der TBS-Schutzgruppe 
auf. Hierzu gehören auch die Signale bei $\delta=18.2$ und $26.0 \mathrm{ppm}$, welche durch die tert-Butylgruppe hervorgerufen werden. Ebenfalls sehr stark abgeschirmt ist das C-9Kohlenstoffatom, das bei $\delta=9.6 \mathrm{ppm}$ resoniert. Die Kohlenstoffatome C-3', C-6' und $\mathrm{C}-1$ besitzen alle eine sehr ähnliche chemische Verschiebung und sind im Bereich von $\delta=18.5-19.4$ ppm zu finden. Etwas weiter zu tiefem Feld verschoben liegt das Signal für C-5 bei $\delta=21.4 \mathrm{ppm}$. Der nächste Block an Signalen $(\delta=29.9-31.4)$, die eine sehr ähnliche Resonanzfrequenz aufweisen, wird von den Kohlenstoffatomen C-4, C-2' und C-8 gebildet. Bei $\delta=36.8$ ppm kann das Signal für C-6 beobachtet werden. Die beiden Methylgruppen der Dimethylaminofunktion resonieren zusammen bei $\delta=40.7 \mathrm{ppm}$. Wesentlich weiter in den Tieffeldbereich verschoben findet man die Signale für die beiden Ringkohlenstoffatome C-4' $(\delta=64.8 \mathrm{ppm})$ und $\mathrm{C}^{-} 5^{\prime}(\delta=74.0 \mathrm{ppm})$, die sich jeweils in unmittelbarer Nähe zu einem Heteroatom befinden. Gleiches gilt für C-2 sowie C-7, die durch den Elektronenzug des benachbarten Sauerstoffatoms wesentlich stärker entschirmt sind als die übrigen aliphatischen Kohlenstoffatome und bei $\delta=69.1$ bzw. 73.4 ppm resonieren. Das Kohlenstoffatom an C-3 besitzt eine größere Resonanzfrequenz und ist bei $\delta=83.7 \mathrm{ppm}$ zu finden. Komplettiert wird der Datensatz des Kohlenstoffspektrums durch das Signal für das Kohlenstoffatom des anomeren Zentrums (C-1'), das eine chemische Verschiebung von $\delta=100.7 \mathrm{ppm}$ aufweist.

Das ESI-Massenspektrum zeigt zwei signifikante Peaks mit $m / z=432.4$ und 885.7. Diese können der Verbindung 103 als $[\mathrm{M}+\mathrm{H}]^{+}$und $[2 \mathrm{M}+\mathrm{H}]^{+}$zugeordnet werden. Darüber hinaus bestätigt die ESI-Hochauflösung die chemische Zusammensetzung mit der Summenformel $\mathrm{C}_{23} \mathrm{H}_{49} \mathrm{NO}_{4} \mathrm{Si}$. 


\section{Untersuchungen zur Kupplung des Tricyclus 60 mit den Bausteinen $\alpha / \beta-103$}

\subsection{Untersuchungen zur 1,2-Addition}

Die Kupplung der beiden Bausteine sollte durch eine 1,2-Addition einer Organolithiumspezies, hergestellt aus dem Bromid $\mathbf{1 2 4}$ an den tricyclischen Aldehyd $\mathbf{6 0}$ erfolgen (vergleiche dazu Abbildung 19). Dazu sollte die freie Alkoholfunktion der beiden Glykoside $\alpha-103$ und $\beta-103$ zunächst unter Inversion des stereogenen Zentrums an dieser Stelle in ein Halogenid überführt werden, welches dann durch einen Halogen-Lithium-Austausch (Retention der Konfiguration) die für die Kupplung benötigte Organolithiumspezies liefern würde. Die Transformation einer Hydroxyfunktion in ein Halogenid $(\mathrm{Cl}, \mathrm{Br}$ und $\mathrm{I})$ unter Inversion der Stereoinformation ist in der Literatur in vielen Beispielen beschrieben. Die wohl am häufigsten verwendeten Bedingungen sind unter dem Namen Appel-Reaktion ${ }^{73}$ bekannt. Jedoch ist es in unserem Fall nicht gelungen, geeignete Reaktionsbedingungen für eine Überführung des sekundären Alkohols in ein Halogenid $(\mathrm{Cl}, \mathrm{Br}$ und $\mathrm{I})$ zu finden. Die ersten Versuche beschränkten sich auf die Einführung eines Bromatoms unter Appel-Bedingungen bzw. Appel-ähnlichen Bedingungen (Tabelle 6). Es wurden sowohl verschiedene Phosphine als auch Bromquellen verwendet. Lediglich in einem Fall (Eintrag 8, Tabelle 6) konnte die gewünschte Verbindung in Spuren in der Reaktionslösung via Massenspektrometrie detektiert werden. Eine Isolierung und Charakterisierung der Substanz war aufgrund der geringen Menge nicht möglich. Darüber hinaus erwies sich das Protokoll als nicht reproduzierbar. 


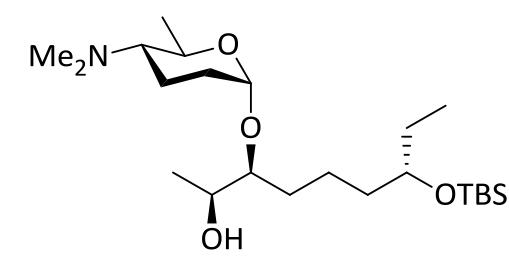

$\alpha-103$

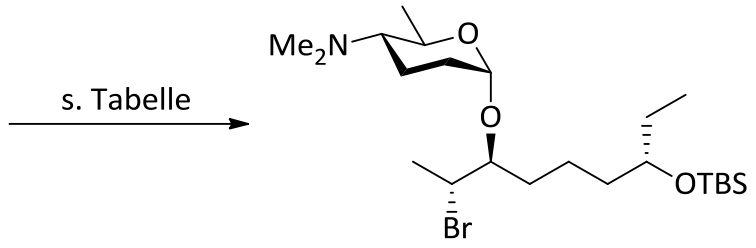

$\alpha-124$

\begin{tabular}{lcc}
\hline$\#$ & Bedingungen & Ergebnis \\
\hline $\mathbf{1}$ & $3.2 \mathrm{Äq} . \mathrm{PPh}_{3}, 3.0 \mathrm{Äq} . \mathrm{CBr}_{4}, \mathrm{CH}_{2} \mathrm{Cl}_{2}, \mathrm{RT}, 2.5 \mathrm{~h}$ & Kein \\
& & Umsatz \\
$\mathbf{2}$ & $4.5 \ddot{\mathrm{Aq}} . \mathrm{PPh}_{3}, 5.0 \mathrm{Äq} . \mathrm{CBr}_{4}$, Benzol, $50{ }^{\circ} \mathrm{C}, 1.5 \mathrm{~h}$ & Zersetzung
\end{tabular}

31.3 Äq. $\mathrm{PPh}_{3}, 1.5$ Äq. NBS, THF, $-15^{\circ} \mathrm{C}, 40 \mathrm{~min}$, dann RT, $40 \mathrm{~min}$

Zersetzung

1.5 Äq. $\mathrm{PMe}_{3}, 1.6 \mathrm{Äq} . \mathrm{CBr}_{4}, \mathrm{CH}_{2} \mathrm{Cl}_{2}, 0^{\circ} \mathrm{C}, 30 \mathrm{~min}$, dann RT, $24 \mathrm{~h}$, dann $40^{\circ} \mathrm{C}, 2 \mathrm{~h}$

Zersetzung

4

$5 \quad 1.5$ Äq. $\mathrm{P}(n \text {-Octyl })_{3}, 1.6$ Äq. $\mathrm{CBr}_{4}, \mathrm{THF}, \mathrm{RT}, 24 \mathrm{~h}$, dann $40^{\circ} \mathrm{C}, 12 \mathrm{~h} \quad$ Zersetzung

64.0 Äq. $\mathrm{P}(n-\mathrm{Octyl})_{3}, 2.0$ Äq. $\mathrm{CBr}_{4}$, Benzol, $50{ }^{\circ} \mathrm{C}, 30 \mathrm{~min}$

Zersetzung

4.0 Äq. P $(n-\mathrm{Octyl})_{3}, 3.0$ Äq. $\mathrm{CBr}_{4}, \mathrm{CH}_{2} \mathrm{Cl}_{2}, 0^{\circ} \mathrm{C}, 1.5 \mathrm{~h}$, dann RT, $19.5 \mathrm{~h}$, dann $40^{\circ} \mathrm{C}, 9 \mathrm{~h}$

Zersetzung

10.0 Äq. P(n-Octyl $)_{3}, 5.0$ Äq. $\mathrm{CBr}_{4}, 10.0$ Äq. Pyridin, Benzol, $70{ }^{\circ} \mathrm{C}$, $16 \mathrm{~h}$

Spuren $^{[\mathrm{a}]}$

2.2 Äq. PPh $3,2.2$ Äq. 2,4,4,6-Tetrabrom-2,5-cyclohexadien-1-on,

$$
\mathrm{CH}_{3} \mathrm{CN}, \mathrm{RT}, 12 \mathrm{~h}
$$

Zersetzung

[a] Detektion via Massenspektrometrie

Tabelle 6: $\quad$ Appel-Bedingungen und Appel-ähnliche Bedingungen zur Darstellung von 124.

Als nächstes wurden andere Bromierungen getestet, die z. T. harscher waren und auch nicht unbedingt ausschließlich unter Inversion der Stereoinformation ablaufen 
sollten. Wie jedoch aus Tabelle $7 \mathrm{zu}$ sehen ist, waren auch diese Methoden nicht erfolgreich.

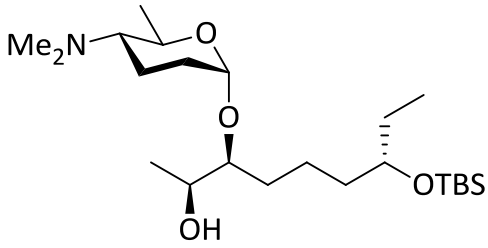

$\alpha-103$

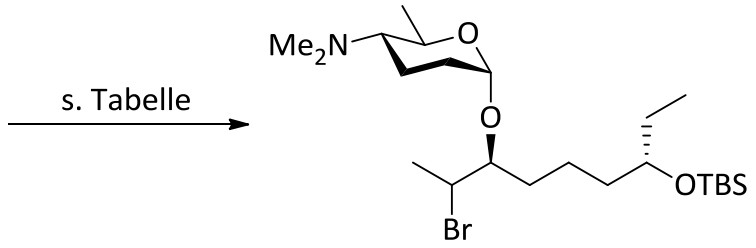

$\alpha-124$

\begin{tabular}{ccc}
\hline$\#$ & Bedingungen & Ergebnis \\
\hline 1 & 1.4 Äq. $\mathrm{PPh}_{3}, 1.4$ Äq. DEAD, 4.0 Äq. LiBr, THF, RT, $20 \mathrm{~h}$ & Kein \\
$\mathbf{2}$ & 2.8 Äq. $\mathrm{PBr}_{3}$, Benzol/THF $(2: 1), 0{ }^{\circ} \mathrm{C}, 1.5 \mathrm{~h}$ & Umsatz
\end{tabular}

310.0 Äq. $\mathrm{SOBr}_{2}, 25.0$ Äq. Pyridin, $\mathrm{CH}_{2} \mathrm{Cl}_{2}, 0{ }^{\circ} \mathrm{C}, 3 \mathrm{~h}$, dann RT, $16 \mathrm{~h} \quad$ Zersetzung

Tabelle 7: $\quad$ Untersuchungen zur Bromierung von $\alpha-103$.

Da der Versuch einer Bromierung unter „Appel-Bedingungen“ offensichtlich nicht möglich war, sollte nun untersucht werden, ob die Einführung eines Halogens über eine andere Methode möglich ist. Hierbei sollte primär ein Sulfonat gebildet werden, das dann in einer nucleophilen Substitution in das Halogenid überführt wird (Tabelle 8). Die Bildung des entsprechenden Mesylats war in geringen Mengen möglich und das Tosylat konnte in Spuren detektiert werden. Allerdings gelang es nicht, diese Verbindungen in die gewünschten Halogenide zu überführen. 


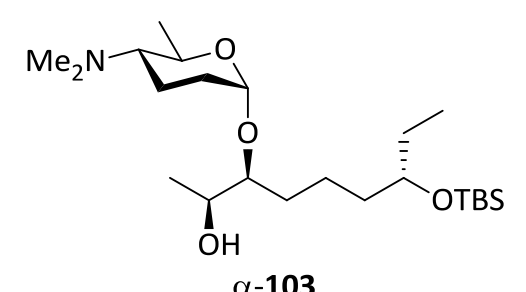

$\alpha-103$

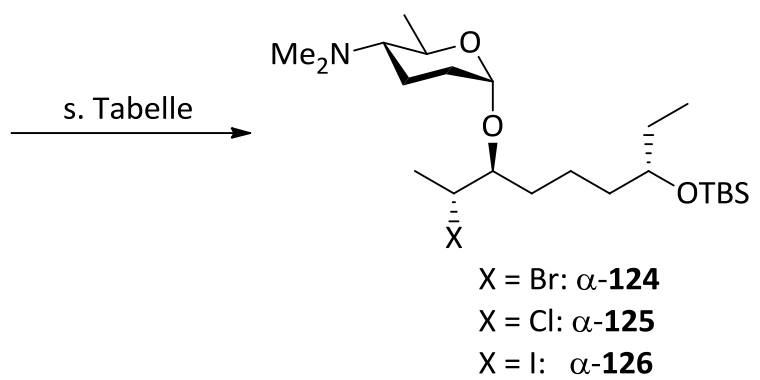

\begin{tabular}{|c|c|c|}
\hline \# & Bedingungen & Ergebnis \\
\hline 1 & $\begin{array}{l}\text { a) } 3.0 \text { Äq. } \mathrm{NEt}_{3}, 1.5 \text { Äq. } \mathrm{TsCl}, \mathrm{CH}_{2} \mathrm{Cl}_{2}, \mathrm{RT}, 20 \mathrm{~h} \\
\text { b) nicht weiter umgesetzt }\end{array}$ & a) in Spuren \\
\hline 2 & $\begin{array}{l}\text { 3.0 Äq. } \mathrm{NEt}_{3}, 10 \mathrm{Mol} \% \mathrm{DMAP}, 1.5 \text { Äq. TsCl, } \mathrm{CH}_{2} \mathrm{Cl}_{2}, \mathrm{RT}, 4 \mathrm{~h} \text {, } \\
\text { dann } 40^{\circ} \mathrm{C}, 15 \mathrm{~h}\end{array}$ & Kein Umsatz \\
\hline 3 & $\begin{array}{l}\text { a) } 3.0 \stackrel{\mathrm{Aq}}{ } \mathrm{NEt}_{3}, 3.2 \mathrm{Äq} . \mathrm{MsCl}, \mathrm{CH}_{2} \mathrm{Cl}_{2}, \mathrm{RT}, 20 \mathrm{~h} \text {, } \\
\text { b) } 2.0 \text { Äq. LiBr, THF, RT, } 48 \mathrm{~h}\end{array}$ & $\begin{array}{l}\text { a) erhalten } \\
\text { b) Kein Umsatz }\end{array}$ \\
\hline 4 & 3.0 Äq. $\mathrm{PPh}_{3}, 9.0$ Äq. $\mathrm{CCl}_{4}, 2.2$ Äq. Imidazol, $\mathrm{CH}_{2} \mathrm{Cl}_{2}, \mathrm{RT}, 24 \mathrm{~h}$ & Kein Umsatz \\
\hline 5 & $\begin{array}{l}\text { a) } 2.8 \text { Äq. } \mathrm{NEt}_{3}, 3.0 \mathrm{Äq} . \mathrm{MsCl}, \mathrm{CH}_{2} \mathrm{Cl}_{2}, \mathrm{RT}, 2 \mathrm{~h} \\
\text { b) } 4.0 \text { Äq. Nal, Aceton, } 60^{\circ} \mathrm{C}, 9 \mathrm{~h}\end{array}$ & $\begin{array}{l}\text { a) erhalten } \\
\text { b) Kein Umsatz }\end{array}$ \\
\hline 6 & $\begin{array}{c}1.5 \text { Äq. } \mathrm{PPh}_{3}, 1.5 \text { Äq. I }, 1.5 \text { Äq. Imidazol, } \mathrm{Et}_{2} \mathrm{O} / \mathrm{CH}_{3} \mathrm{CN}(3: 1) \text {, } \\
\text { RT, } 24 \mathrm{~h}\end{array}$ & Kein Umsatz \\
\hline
\end{tabular}

Tabelle 8:

Synthese eines Halogenids ausgehend von $\alpha-103$.

Es werden an dieser Stelle nur die Ergebnisse der Reaktionen des $\alpha$-Glykosids-103 dargestellt und diskutiert. Die Reaktivität des $\beta$-Glykosids-103 unterschied sich in ausgewählten Testreaktionen nicht und wird deshalb hier nicht explizit beschrieben.

\subsection{Untersuchungen zur Umpolung des Aldehyds 60}

Da die Einführung eines Halogens und somit ein späterer Halogen-Lithium-Austausch nicht umzusetzen war, wurde eine neue Strategie gesucht, um die beiden Bausteine miteinander zu verbinden. Der neue Syntheseplan sah vor, die Reaktivität des Aldehyds durch eine Umpolung ${ }^{74}$ umzukehren. Hierzu müsste zusätzlich die freie Hydroxyfunktion der C-9-Bausteine in eine gute Abgangsgruppe überführt werden, um einen nucleophilen Angriff des umgepolten Aldehyds zu ermöglichen. In der Literatur findet man für die Umpolung der Reaktivität vor allem zwei interessante 
Methoden. Die Umpolung nach Corey-Seebach ${ }^{75}$ erfolgt durch die Bildung eines Dithians mit anschließender Deprotonierung am ehemaligen Carbonylkohlenstoffatom mit einer Lithium-Base, die zur Bildung eines Carbanions führt. Der zweiten Methode, auch als Hünig-Stork-Umpolung bekannt, liegt eine Umwandlung der Carbonylfunktion in ein TMS-Cyanhydrin zugrunde, welches dann ebenfalls durch Deprotonierung mit einer Lithium-Base in ein Carbanion überführt wird. ${ }^{76}$

Die Anwendung dieser beiden Methoden für die Umpolung eines Aldehyds vom Typ 60 wurde bereits mit ähnlich komplexen Molekülen in der Literatur beschrieben. $^{77}$

Wie aus Tabelle 9 zu sehen ist, war es tatsächlich möglich ein Dithian des Typs 127 zur Reaktivitätsumpolung des Aldehyds zu synthetisieren. Unter Lewis-sauren Bedingungen konnte der Aldehyd 60 mit 1,3-Propandithiol in das (S,S)-Acetal 127 mit 24\% Ausbeute überführt werden. Die Synthese des Cyanhydrins 128 war hingegen nicht erfolgreich. 


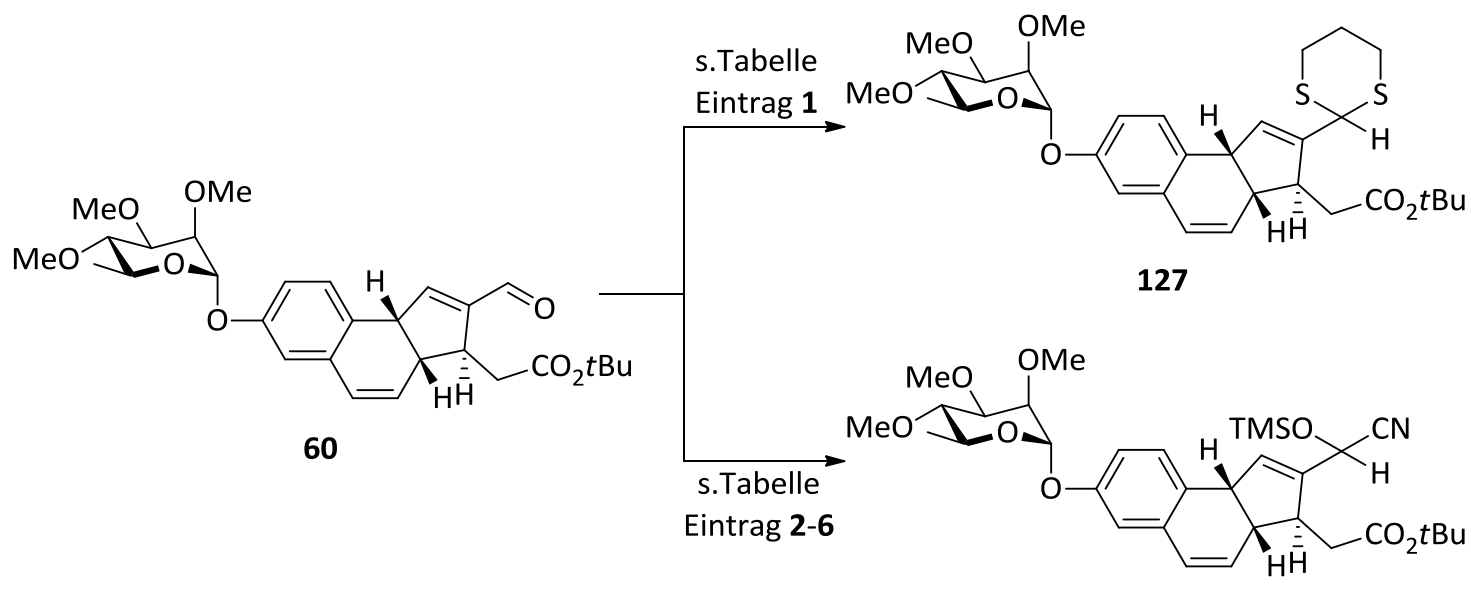

128

\begin{tabular}{ccc}
\hline$\#$ & Bedingungen & Ergebnis \\
\hline \multirow{2}{*}{1} & 1.3 Äq. 1,3-Propandithiol, 0.5 Äq. $\mathrm{BF}_{3} \cdot \mathrm{Et}_{2} \mathrm{O}, \mathrm{Et}_{2} \mathrm{O},-65^{\circ} \mathrm{C}, 16 \mathrm{~h}$, dann & $24 \%$ \\
& $-20{ }^{\circ} \mathrm{C}, 2 \mathrm{~h}$ & \\
\hline
\end{tabular}

21.3 Äq. TMSCN, 2 Mol\% KCN, 2 Mol\% 18-Krone-6, $\mathrm{CH}_{2} \mathrm{Cl}_{2}, \mathrm{RT}, 25 \mathrm{~h}$

Kein

Umsatz

Kein

31.3 Äq. TMSCN, 10 Mol\% KCN, 10 Mol\% 18-Krone-6, $\mathrm{CH}_{2} \mathrm{Cl}_{2}, \mathrm{RT}, 3 \mathrm{~h}$

Umsatz

4

1.3 Äq. TMSCN, 20 Mol\% KCN, 20 Mol\% 18-Krone-6, $\mathrm{CH}_{2} \mathrm{Cl}_{2}, 0^{\circ} \mathrm{C}$,

$$
\text { dann } 3 \mathrm{~h}, \mathrm{RT}, 14 \mathrm{~h}
$$

Zersetzung

1.1 Äq. TMSCN, 10 Mol\% DMAP, 2.5 Äq. $\mathrm{NEt}_{3}, \mathrm{CH}_{3} \mathrm{CN}, \mathrm{RT}, 23 \mathrm{~h}$,

Kein

5

dann 1.1 Äq. TMSCN, $50^{\circ} \mathrm{C}, 18 \mathrm{~h}$

Umsatz

6

1.4 Äq. TMSCN, $10 \mathrm{Mol} \% \mathrm{ZnI}_{2}, \mathrm{CH}_{2} \mathrm{Cl}_{2}, \mathrm{RT}, 4 \mathrm{~h}$

Zersetzung

\section{Tabelle 9: $\quad$ Untersuchungen zur Umpolung des Aldehyds 60.}

Um die Kupplung der Bausteine durchführen zu können, musste nach der erfolgreichen Umpolung des Aldehyds noch die Überführung der Hydroxyfunktion der C-9Bausteine $\alpha-103$ und $\beta-103$ in eine gute Abgangsgruppe bewältigt werden. Wie bereits zuvor beschrieben, war es nicht möglich, den Alkohol in ein Tosylat zu überführen. Eine Synthese des Mesylats war zwar erfolgreich, aber eine Umsetzung des Mesylats unter den späteren Reaktionsbedingungen der Kupplung hätte zu einer Deprotonierung eines der relativ aziden Protonen an der Methylgruppe geführt. Eine weitere, in der Literatur weit verbreitete Abgangsgruppe stellt das Triflat dar. Die 
Versuche zur Herstellung eines solchen Triflats unter verschiedenen Bedingungen sind in Tabelle 10 aufgeführt.

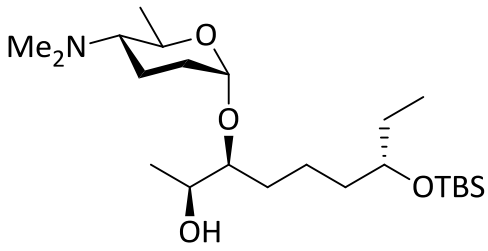

$\alpha-103$

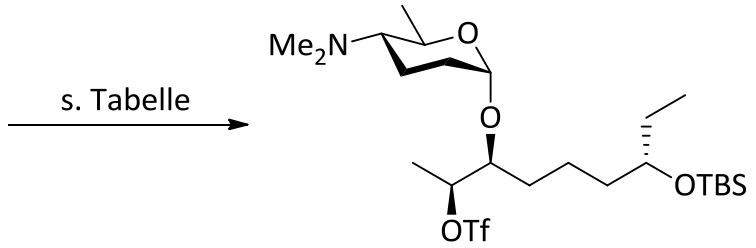

$\alpha-129$

\begin{tabular}{|c|c|c|}
\hline$\#$ & Bedingungen & Ergebnis \\
\hline 1 & 5.0 Äq. Pyridin, 1.3 Äq. $\mathrm{Tf}_{2} \mathrm{O}\left(1 \mathrm{M}\right.$ in $\left.\mathrm{CH}_{2} \mathrm{Cl}_{2}\right), \mathrm{CH}_{2} \mathrm{Cl}_{2}, \mathrm{RT}, 1 \mathrm{~h}$ & $\begin{array}{l}\text { Kein } \\
\text { Umsatz }\end{array}$ \\
\hline 2 & $\begin{array}{l}\text { 2.0 Äq. Pyridin, } 1.3 \ddot{A} q . \mathrm{Tf}_{2} \mathrm{O}\left(1 \mathrm{M} \text { in } \mathrm{CH}_{2} \mathrm{Cl}_{2}\right), 10 \mathrm{Mol} \% \text { DMAP, } \\
\qquad \mathrm{CH}_{2} \mathrm{Cl}_{2}, \mathrm{RT}, 4 \mathrm{~h}\end{array}$ & $\begin{array}{l}\text { Kein } \\
\text { Umsatz }\end{array}$ \\
\hline 3 & 5.0 Äq. Pyridin, 1.3 Äq. Tf ${ }_{2} \mathrm{O}$ (rein), 10 Mol\% DMAP, $\mathrm{CH}_{2} \mathrm{Cl}_{2}, \mathrm{RT}, 4 \mathrm{~h}$ & $\begin{array}{l}\text { Kein } \\
\text { Umsatz }\end{array}$ \\
\hline 4 & $\begin{array}{l}1.8 \text { Äq. 1-(Trifluormethansulfonyl)imidazol, } 10 \text { Mol\% Imidazol, } \\
\qquad \mathrm{CH}_{2} \mathrm{Cl}_{2}, \mathrm{RT}, 4 \mathrm{~h} \text {, dann } 40{ }^{\circ} \mathrm{C}, 13 \mathrm{~h}\end{array}$ & $\begin{array}{l}\text { Kein } \\
\text { Umsatz }\end{array}$ \\
\hline
\end{tabular}

Tabelle 10: Untersuchungen zur Darstellung des Triflats 129.

Unglücklicherweise war auch die Synthese eines Triflats nicht möglich, was dazu führte, dass keine weiteren Untersuchungen zur Optimierung der Umpolung durchgeführt wurden. Dementsprechend musste auch dieser Verknüpfungsversuch der beiden Bausteine als gescheitert betrachtet werden. Darüber hinaus war es nicht möglich, noch andere Kupplungsmethoden zu erforschen, da die vorhandenen Substanzmengen dafür nicht ausgereicht hätten. Die neuerliche Synthese des Bausteins oder anderer Derivate hätte im zeitlichen Rahmen dieser Arbeit nicht mehr stattfinden können. Der Grund dafür, warum die Manipulation des sekundären Alkohols auf so viele verschiedene Weisen nicht funktionierte, konnte nicht abschließend geklärt werden und ist rein spekulativer Natur. Es wird vermutet, dass die sekundäre Alkoholfunktion durch die Anwesenheit der sterisch sehr anspruchsvollen Zuckereinheit in der Nachbarposition stark abgeschirmt und somit für Reaktionen jeglicher Art schwer zugänglich ist. 


\section{E. ZUSAMMENFASSUNG}

Die in dieser Arbeit vorgestellte Naturstoffklasse der Spinosyne besitzt eine hohe insektizide Wirkung und ein 85:15-Gemisch von Spinosyn A (30) und D (31) wird im Pflanzenschutz eingesetzt. Diese als Hauptmetabolite der Streptomyces-Spezies Saccharopolyspora spinosa isolierten Verbindungen besitzen eine hohe Selektivität und Aktivität gegenüber einer Vielzahl von Schadorganismen. Aufgrund ihres hervorragenden ökologischen Profils werden die Verbindungen auch in der Veterinärmedizin zum Schutz von Nutz- und Haustieren vor Parasiten sowie in der MalariaProphylaxe verwendet.

Aufgrund von ersten Resistenzerscheinungen bei einzelnen Schädlingsklassen zu Beginn des neuen Jahrtausends ist es allerdings im Rahmen eines verantwortungsvollen Resistenzmanagements dringend erforderlich, neuartige Spinosynanaloga bereitzustellen. Dabei kann die Synthesechemie eine wichtige Rolle spielen, vor allem wenn man bedenkt, dass die Spinosyne für die industrielle Anwendung bis heute ausschließlich durch aufwändige Fermentation gewonnen werden. Die literaturbekannten Totalsynthesen sind jedoch für die industrielle Anwendung nicht geeignet, da sie zum einen zu viele Syntheseschritte benötigen und zum anderen wenig Spielraum für Derivatisierungen lassen. Darüber hinaus muss es im Hinblick auf Effizienz und Wirtschaftlichkeit das Ziel sein, die Komplexität der natürlichen Struktur durch die Herstellung vereinfachter Spinosynanaloga zu verringern.

In dieser Arbeit wird daher die Synthese der Bausteine 60 und 103 sowie erste Untersuchungen zu deren Kupplung beschrieben, um ein Spinosynanalogon vom Typ 58 mit modifiziertem Ring A aufzubauen (Abbildung 45). Die Einführung eines aromatischen Rings anstelle der aliphatischen Cyclopentaneinheit erschien sinnvoll, da Untersuchungen zu Struktur-Aktivitäts-Beziehungen gezeigt haben, dass die Einführung einer zusätzlichen $C=C$-Doppelbindung in Ring $A$, welche zu einer Verringerung der Anzahl stereogener Zentren führt, keine negativen Auswirkungen auf die biologische Aktivität hat. 


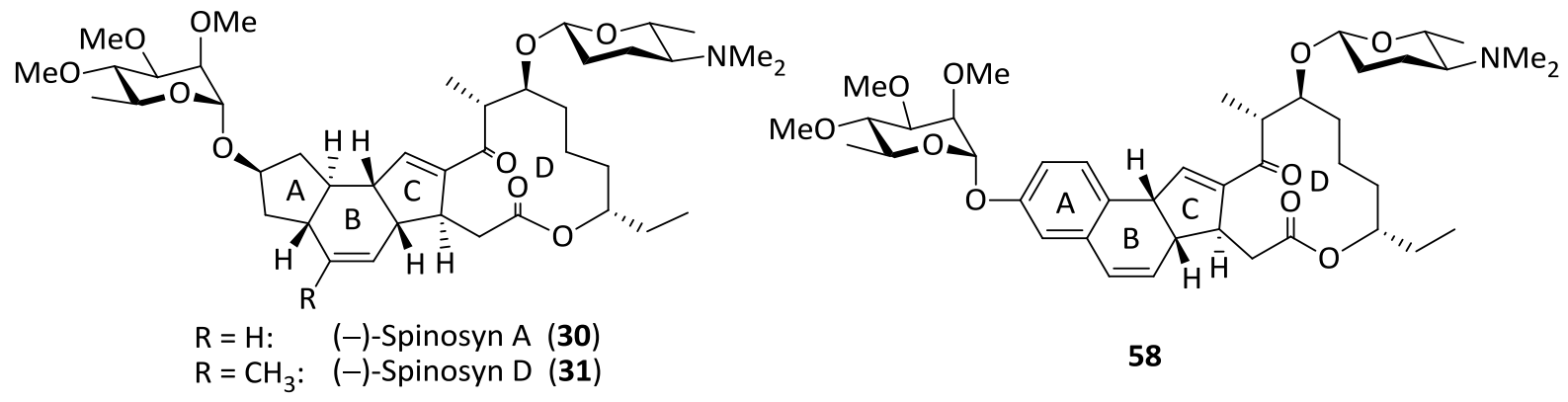

Abbildung 45: $\quad$ Natürliche Spinosyne A (30) und D (31) sowie Spinosynanalogon 58.

Ziel dieser Arbeit war die Entwicklung von Bausteinen für eine neue konvergente Syntheseroute zum Aufbau eines Spinosynanalogons vom Typ 58, bei der eine möglichst frühe Einführung der für die Wirkung des Moleküls essentiellen Forosamineinheit im Vordergrund stand. In Zusammenarbeit mit S. Dietz aus dem Arbeitskreis Tietze wurde daher die $\beta$-selektive Glykosidierung des Bausteins 61 und dessen Vorläufer mit enantiomerenreinem D-Forosamin (68) untersucht (Abbildung 46).
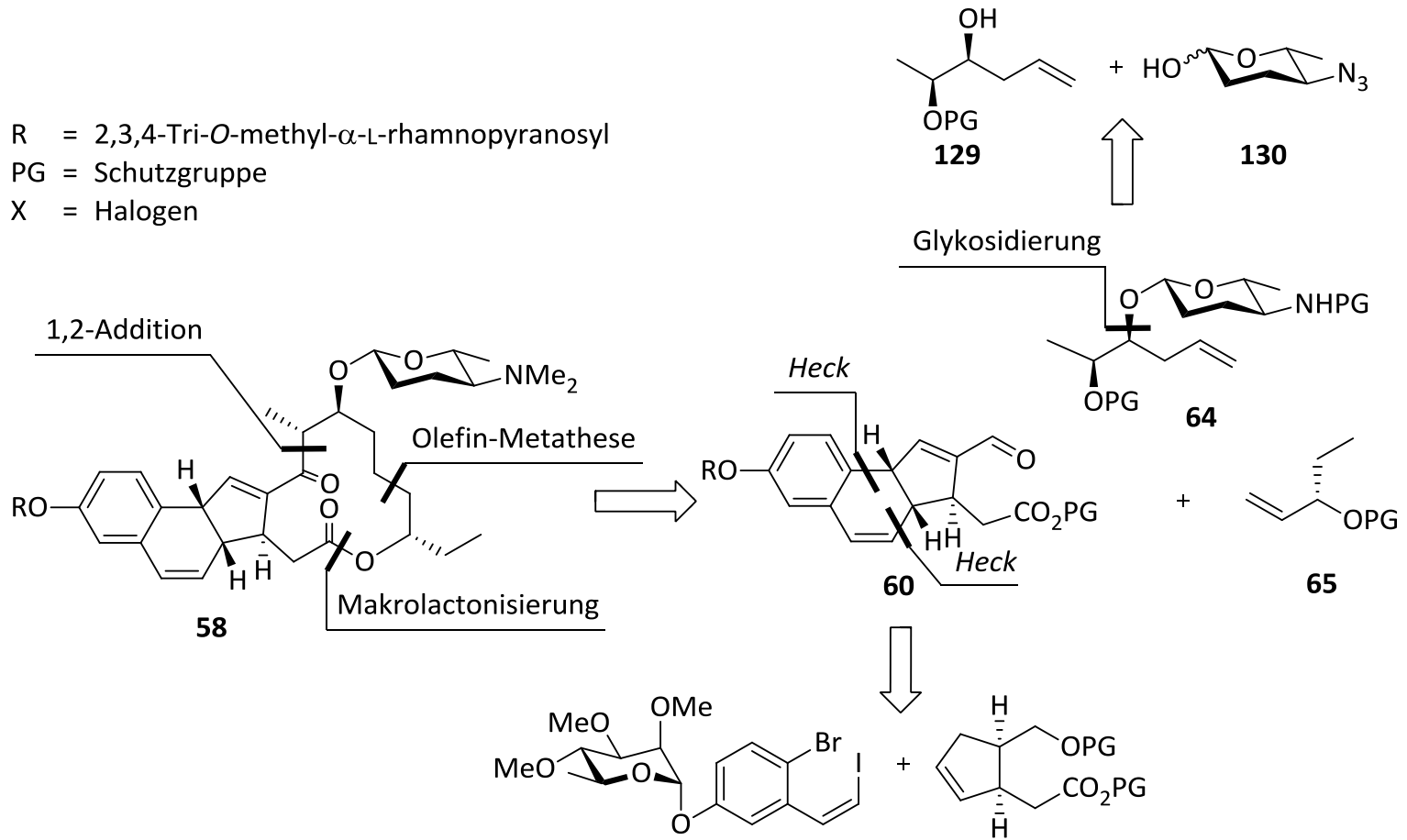

62

63

Abbildung 46: $\quad$ Retrosyntheseschema zum Aufbau eines Spinosynanalogons vom Typ 58.

Das tricyclische Grundgerüst 60 mit cis-Verknüpfung des B-C-Ringsystems ließ sich dabei durch zwei hintereinander geschaltete Heck-Reaktionen aufgebauen. Dazu wurden ein cis-1,2-disubstituiertes Cyclopenten vom Typ 63 und ein bereits die Rhamnoseeineit tragendes Vinyliodid 62 benötigt. Wie das Retrosyntheseschema in 
Abbildung 46 zeigt, sollte die Herstellung des benötigten $\beta$-Glykosids 61 durch eine Olefin-Metathese zwischen dem Hexen 64 und dem enantiomerenreinen C-5Fragment 65 erfolgen. Die Kupplung der beiden Bausteine 60 und 61 würde dann auf einer 1,2-Addition basieren, wobei geplant war, dass der Baustein 61 als Lithiumorganyl an der Aldehydfunktion des Tricyclus 60 angreift.

Als literaturbekannte Vorstufe für den Aufbau des cis-1,2-disubstituierten Cyclopentenbausteins wurde der leicht auch im Multigrammmaßstab herzustellende Bicyclus 79 eingesetzt (Abbildung 47). Ausgehend von Malonsäuredimethylester (73) und Cyclopentadien (77) erfolgte die Synthese in nur vier Stufen mit einer [2+2]Cycloaddition als Schlüsselschritt, welche die cis-Verknüpfung der Substituenten gewährleistete. Der Bicyclus 79 wurde anschließend in einer Dominoreaktion, die aus einer Reduktions/Retroaldol/Reduktions-Sequenz bestand, zu dem Lacton 83 umgesetzt. Überführung in den tert-Butylester 69 und nachfolgende Schützung der primären Alkoholfunktion als TBS-Ether lieferte den ersten Baustein für die zweifache Heck-Reaktion zum Aufbau des tricyclischen Grundgerüsts.

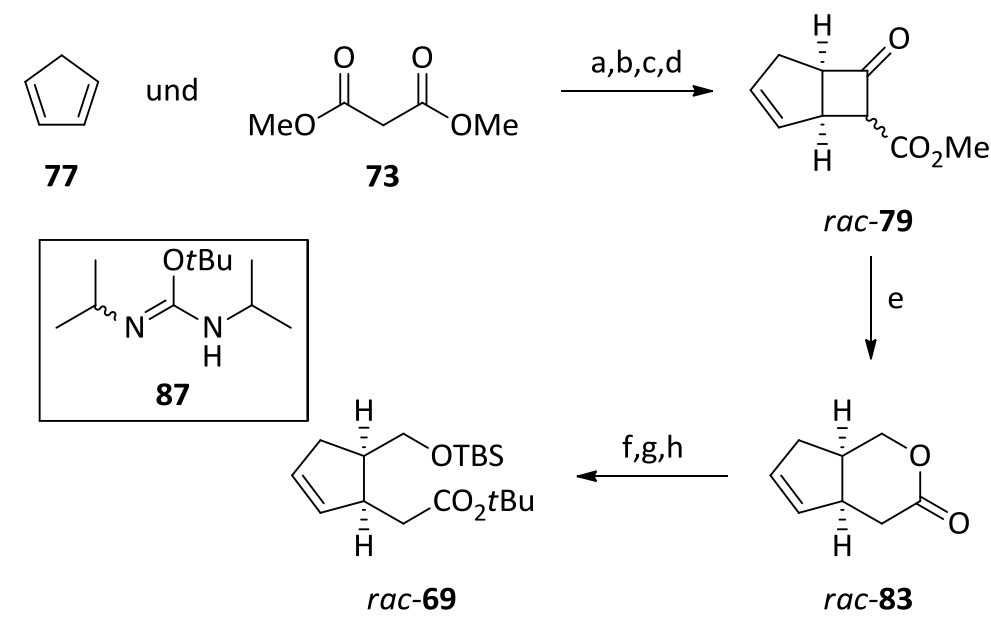

Abbildung 47: Synthese des cis-1,2-disubstituierten Cyclopentenbausteins 69: a) $\mathrm{KOH}, \mathrm{MeOH}, \mathrm{RT}$, $24 \mathrm{~h}$, dann Rückfluss, $1 \mathrm{~h}, 70 \%$; b) $\mathrm{SOCl}_{2}, \mathrm{NCS}, 70^{\circ} \mathrm{C}, 30 \mathrm{~min}$, dann $85^{\circ} \mathrm{C}, 100 \mathrm{~min}$, 76\%; c) NEt 3 , 77, n-Hexan, RT, 14 h, 70\% (roh); d) Zn-Staub, konz. HOAc, RT, 75 min, 78\%; e) $\mathrm{NaBH}_{4}, \mathrm{MeOH}, 0{ }^{\circ} \mathrm{C}, 45 \mathrm{~min}, 88 \%$; f) $\mathrm{NaOH}, \mathrm{MeOH}$, Rückfluss, 5 h; g) $\mathrm{NH}_{4} \mathrm{Cl}$, 87, $\mathrm{CH}_{2} \mathrm{Cl}_{2} / t$-BuOH, RT, 19 h, 79\% (über 2 Stufen); h) TBSCl, Imidazol, DMF, RT, 2 h, $85 \%$.

Die Herstellung des aromatischen Fragments 62 begann zunächst mit der Synthese des für die Glykosidierung benötigten Trichloracetimidats 92, welches in einer 
Gesamtausbeute von $47 \%$ erhalten wurde. Ausgangsverbindung für die vierstufige Sequenz war das kommerziell erhältliche L-Rhamnose-Monohydrat.

Der literaturbekannte Glykosylakzeptor 66 war über eine selektive Bromierung und anschließende Methylentschützung in guter Ausbeute ausgehend vom käuflich zu erwerbenden 3-Methoxybenzaldehyd (94) zugänglich (Abbildung 48).

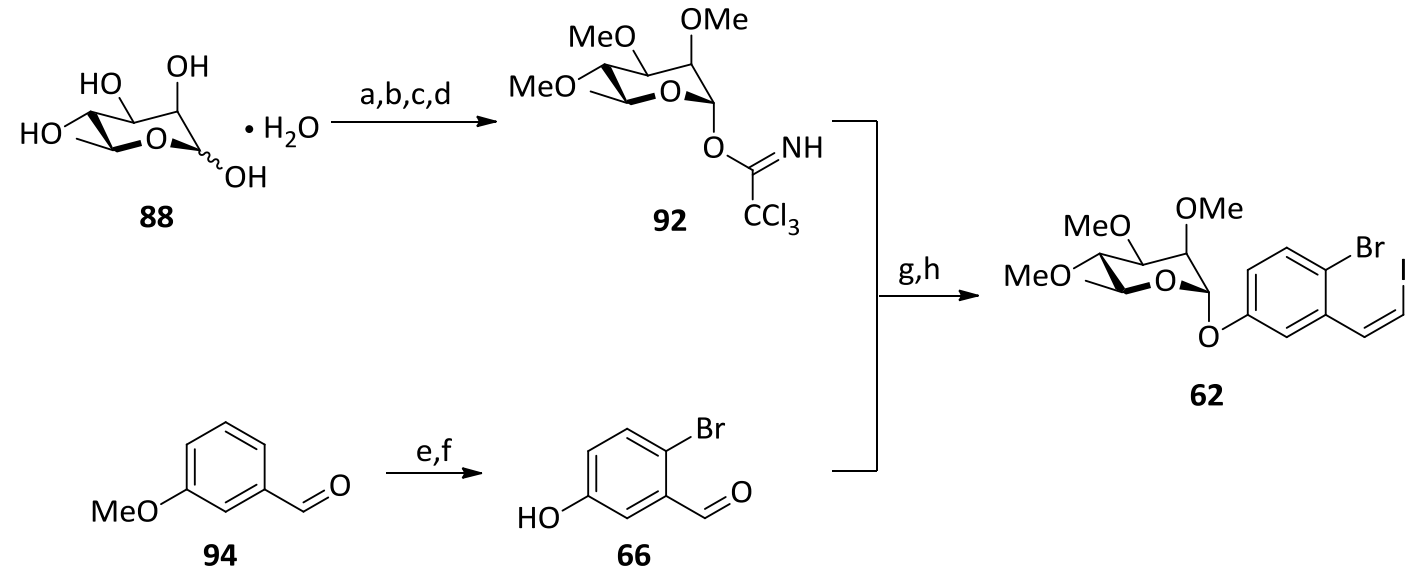

Abbildung 48: Synthese des Vinyliodids 62: a) Amberlite IR 120, MeOH, Rückfluss, 48 h, quant.; b) $\mathrm{NaH}$, Mel, DMSO, RT, $20 \mathrm{~h}, 48 \%$; c) $0.5 \mathrm{M} \mathrm{H}_{2} \mathrm{SO}_{4}, 100{ }^{\circ} \mathrm{C}, 19 \mathrm{~h}, 98 \%$; d) $\mathrm{CCl}_{3} \mathrm{CN}$, PS-DBU, $\mathrm{CH}_{2} \mathrm{Cl}_{2}, \mathrm{RT}, 20$ min, 99\%; e) $\mathrm{Br}_{2}, \mathrm{CH}_{2} \mathrm{Cl}_{2}, 0{ }^{\circ} \mathrm{C} \rightarrow \mathrm{RT}, 18 \mathrm{~h}, 78 \%$; f) $\mathrm{BBr}_{3}, \mathrm{CH}_{2} \mathrm{Cl}_{2}$, $-20{ }^{\circ} \mathrm{C}, 22 \mathrm{~h}, 77 \%$; g) 66, 92, TMSOTf (kat.), MS $4 \AA$, $0^{\circ} \mathrm{C}, \mathrm{CH}_{2} \mathrm{Cl}_{2}, 75 \mathrm{~min}, 88 \%$; h) $\left[\mathrm{Ph}_{3} \mathrm{PCH}_{2} \mathrm{I}^{+} \mathrm{I}^{-}, \mathrm{KHMDS}, \mathrm{THF},-78^{\circ} \mathrm{C}, 1 \mathrm{~h}\right.$, dann RT, $45 \mathrm{~min}, 78 \%$.

Die anschließende Lewis-Säure-katalysierte Glykosidierung mit TMSOTf gelang ebenfalls in sehr guter Ausbeute von 88\% unter Erhalt des $\alpha$-Glykosids 96. Eine abschließende Wittig-Reaktion führte zu dem für die Heck-Reaktion benötigten (Z)-Isomer des Vinyliodids 62.

Die zweifache Heck-Reaktion, zwischen dem Vinyliodid 62 und dem Cyclopenten 69, gelang insgesamt in 46\% Ausbeute, wobei das gewünschte Kupplungsprodukt 100 als 2:1-Gemisch der Diastereomere isoliert wurde (Abbildung 49). Nach der Entschützung der primären Alkoholfunktion konnten die Diastereomere mittels HPLC an chiraler stationärer Phase getrennt werden. Abschließende Oxidation mit Dess-MartinPeriodinan lieferte den tricyclischen Baustein 60 in einer Gesamtausbeute von 40\% über vier Stufen. 


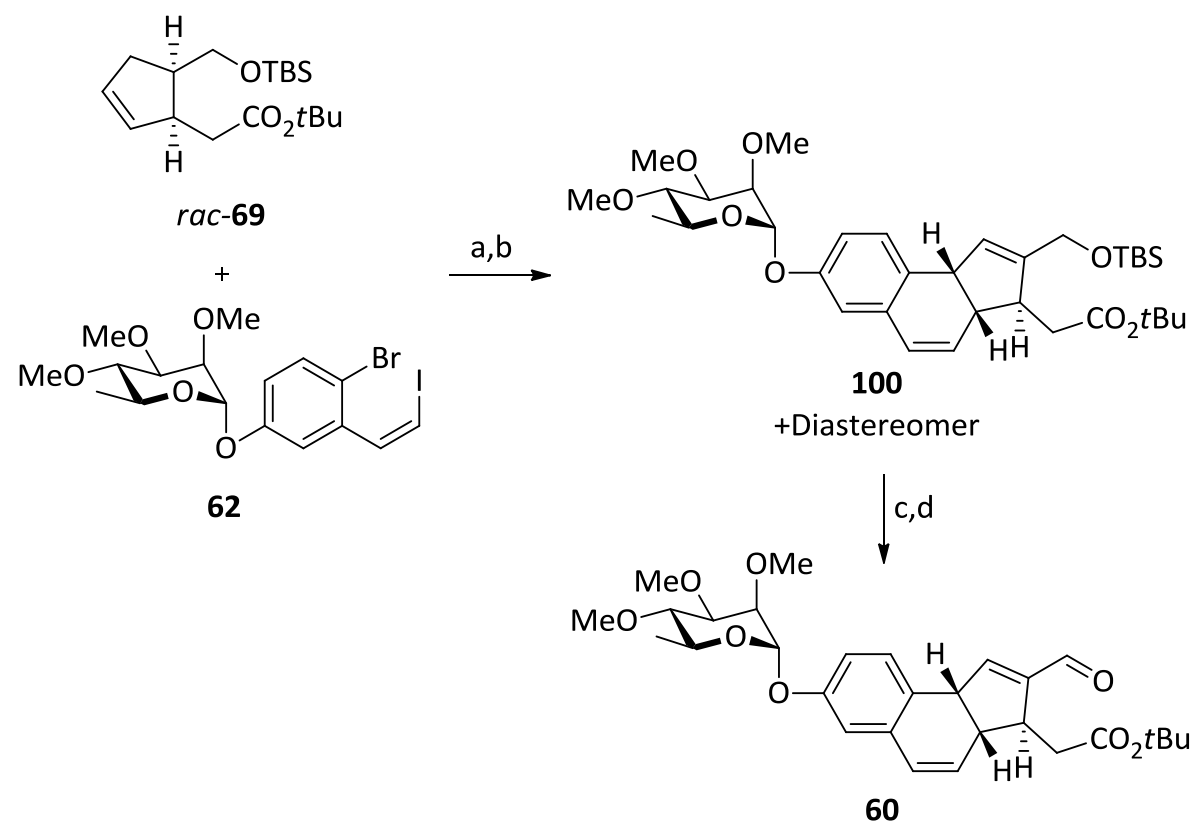

Abbildung 49: Synthese des tricyclischen Grundgerüsts 60: a) 62, 69, $\mathrm{Pd}(\mathrm{OAc})_{2}$ (kat.), $\mathrm{NaOAc}$, $n-\mathrm{Bu}_{4} \mathrm{NCl}, \mathrm{DMF}, \mathrm{RT}, 20 \mathrm{~h}, 48 \%$; b) Herrmann-Beller-Katalysator (101) (kat.), $n$-Bu $\mathrm{BuAC}_{4} \mathrm{DMF} / \mathrm{CH}_{3} \mathrm{CN} / \mathrm{H}_{2} \mathrm{O}, 130{ }^{\circ} \mathrm{C}, 3 \mathrm{~h}, 96 \%$; c) $p$ - $\mathrm{TsOH}$ (kat.), $\mathrm{MeOH}, 0{ }^{\circ} \mathrm{C}, 4.5 \mathrm{~h}$, 97\%; d) DMP, $\mathrm{CH}_{2} \mathrm{Cl}_{2}, 0^{\circ} \mathrm{C}, 1.5 \mathrm{~h}, 89 \%$.

Die C-9-Kette für die Synthese des Bausteins 103 wurde über eine Olefin-Metathese des C-5-Fragments 118 mit dem Hexen 115 synthetisiert. Der Aufbau des enantiomerenreinen C-5-Bausteins erfolgte ausgehend vom kommerziell erhältlichen rac-1-Penten-3-ol (116) durch eine enzymatische Racematspaltung mit anschließender Veresterung durch 3,5-Dinitrobenzoylchlorid. Über zwei Stufen wurde so das (S)-Dinitrobenzoat 118 in $23 \%$ Ausbeute und $81 \%$ ee erhalten. Durch präparative HPLC an chiraler stationärer Phase konnte der Enantiomerenüberschuss auf über 99\% erhöht werden.

Für die Synthese des Hexens 115 wurde zunächst das käuflich zu erwerbende (S)-Methyllactat in einer dreistufigen Synthesesequenz in das Hexenol 107 überführt. Schlüsselschritt dabei war eine Lewis-Säure-vermittelte Allylierung, die über CramChelat-Kontrolle hochdiastereoselektiv zum syn-Produkt führte. Die darauffolgende Glykosidierung mit dem Forosaminderivat 113 wurde von S. Dietz aus dem Arbeitskreis Tietze im Rahmen ihrer Dissertation untersucht und lieferte die Glykoside in einem Verhältnis von $\alpha / \beta=7: 5$ (Abbildung 50). Auch die beiden anschließenden Transformationen, welche die für die Metathese benötigten Bausteine $\alpha-\mathbf{1 1 5}$ und 
ß-115 lieferten, waren Bestandteil der Arbeit. Durch säulenchromatographische Trennung an Kieselgel war die Trennung der beiden Anomere möglich.<smiles>C=CC(O)CC</smiles>

$\operatorname{rac}-116$

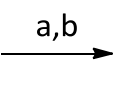<smiles>C=C[C@H](CC)OC(=O)c1cc([N+](=O)[O-])cc([N+](=O)[O-])c1</smiles>

118

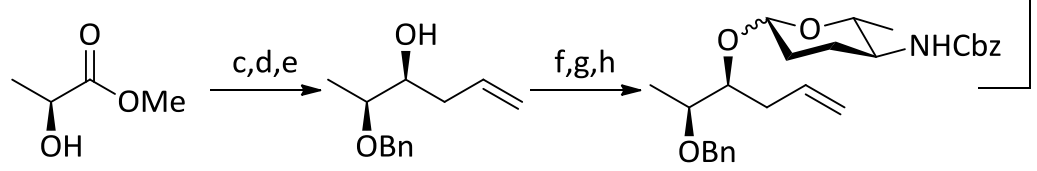

$\alpha / \beta-115$

107

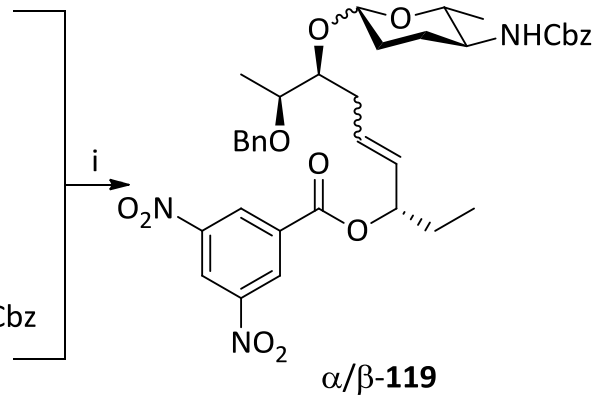

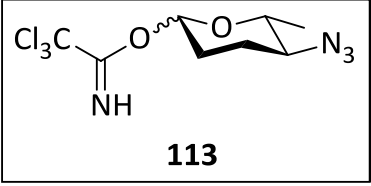

Abbildung 50: Aufbau der C-9-Fragmente $\alpha-119$ und $\beta$-119: a) Amano Lipase AK, Vinylacetat,

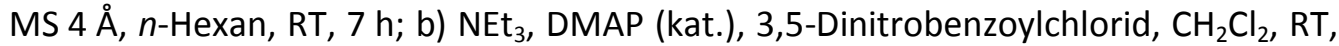
14 h, 23\% (über 2 Stufen); c) Benzyl-2,2,2-trichloracetimidat, TfOH (kat.), Cyclohexan $/ \mathrm{CH}_{2} \mathrm{Cl}_{2}, \mathrm{RT}, 8 \mathrm{~h}, 83 \%$; d) DIBAL, $\mathrm{Et}_{2} \mathrm{O},-78{ }^{\circ} \mathrm{C}, 20 \mathrm{~min}, 92 \%$; e) $\mathrm{SnCl}_{4}$, Allyltrimethylsilan, $-78{ }^{\circ} \mathrm{C}, 20 \mathrm{~min}, 81 \%$; f) 113, $\mathrm{Et}_{3} \mathrm{SiH}, \mathrm{I}_{2}, \mathrm{MS} 5 \AA$, Toluol, $-90{ }^{\circ} \mathrm{C}, 2.5 \mathrm{~h}, 96 \%$ $(\alpha / \beta=7: 5)$; g) $\mathrm{PMe}_{3}, \mathrm{NaOH}, \mathrm{H}_{2} \mathrm{O}, \mathrm{THF}, \mathrm{RT}, 3.5 \mathrm{~h}, 90 \%$; h) $\mathrm{CbzCl}, \mathrm{K}_{2} \mathrm{CO}_{3}, \mathrm{CH}_{2} \mathrm{Cl}_{2}, \mathrm{RT}$, 12 h, 96\%; i) 115, 118, Grubbs-Katalysator 2. Generation (kat.), $\mathrm{CH}_{2} \mathrm{Cl}_{2}$, Rückfluss, 1826 h, 74\% für $\alpha-119$ ((E/Z = 7:1), 70\% für $\beta-119(E / Z=7: 1)$.

Die Kupplung der Bausteine via Olefin-Metathese führte dann in beiden Fällen zu einem Gemisch der Doppelbindungsisomere $(E: Z=7: 1)$, was aber angesichts der später erforderlichen Hydrierung der Doppelbindung kein Problem darstellte. Im nächsten Schritt der Synthesesequenz wurde dann die nicht mehr benötigte Dinitrobenzoatgruppe durch die TBS-Schutzgruppe ersetzt, da diese eine größere Zahl an Reaktionsbedingungen toleriert und somit für den weiteren Verlauf der Synthese besser geeignet war. Nun erfolgte die Hydrierung der Doppelbindung in Gegenwart der Cbz- und Benzylschutzgruppe unter Erhalt der Glykoside $\alpha-122$ (77\% über 3 Stufen) und $\beta-122$ (61\% über 3 Stufen)(Abbildung 51). 


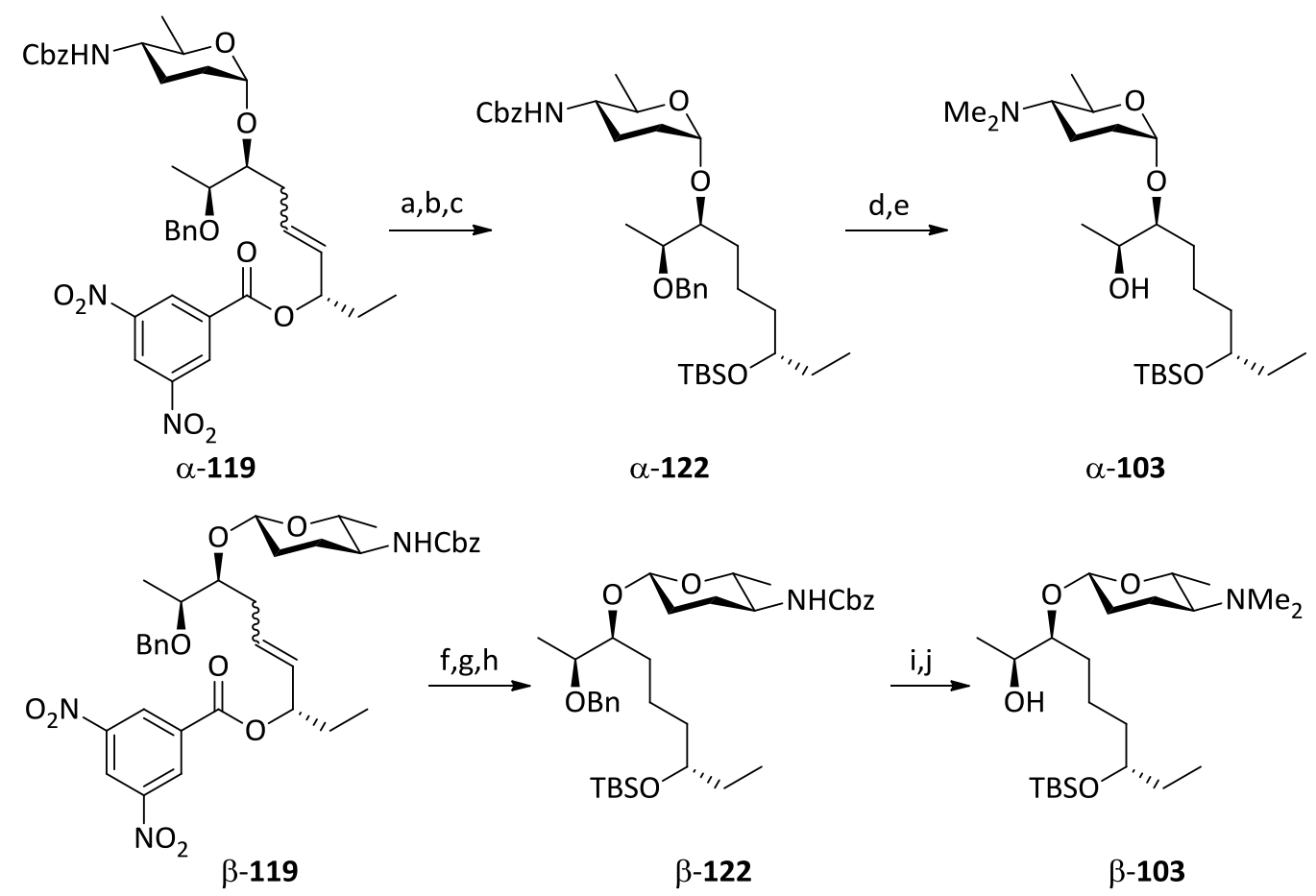

Abbildung 51: Synthese der Bausteine $\alpha-103$ und $\beta-103:$ a) $\mathrm{K}_{2} \mathrm{CO}_{3}, \mathrm{MeOH} / \mathrm{THF}, 0{ }^{\circ} \mathrm{C}, 45 \mathrm{~min}, 93 \%$ $(E / Z=7: 1)$; b) 2,6-Lutidin, TBSOTf, $\mathrm{CH}_{2} \mathrm{Cl}_{2},-10^{\circ} \mathrm{C}, 30 \mathrm{~min}, 94 \%(E / Z=7: 1)$; c) Pt/C (kat.), $\mathrm{H}_{2}$ (1 atm), EtOH / $\mathrm{CH}_{2} \mathrm{Cl}_{2}, \mathrm{RT}, 2 \mathrm{~h}, 88 \%$; d) Pd/C (kat.), $\mathrm{H}_{2}$ (3.8 bar), Formalin, $\mathrm{MeOH} / \mathrm{EtOAc} / \mathrm{CH}_{2} \mathrm{Cl}_{2}, \mathrm{RT}, 20 \mathrm{~h}, 82 \%$; e) Li, $\mathrm{NH}_{3(1)}$, THF, $-78 \mathrm{C}, 1 \mathrm{~h}, 91 \%$; f) $\mathrm{K}_{2} \mathrm{CO}_{3}$, $\mathrm{MeOH} / \mathrm{THF}, 0{ }^{\circ} \mathrm{C}, 45 \mathrm{~min}, 89 \%(E / Z=7: 1) ;$ g) 2,6-Lutidin, TBSOTf, $\mathrm{CH}_{2} \mathrm{Cl}_{2},-10{ }^{\circ} \mathrm{C}$, 35 min, 93\% (E/Z = 7:1); h) $\mathrm{PtO}_{2}$ (kat.), $\mathrm{H}_{2}$ (1 atm), EtOH, RT, 2 h, 74\%; i) Pd/C (kat.), $\mathrm{H}_{2}$ (3.8 bar), Formalin, $\mathrm{MeOH} / \mathrm{EtOAc} / \mathrm{CH}_{2} \mathrm{Cl}_{2}, \mathrm{RT}, 22 \mathrm{~h}, 80 \%$; j) Li, $\mathrm{NH}_{3(1)}, \mathrm{THF},-78 \mathrm{C}, 1 \mathrm{~h}$, $86 \%$.

Anschließend wurde in einem Schritt die Cbz-Schutzgruppe entfernt und das freie Amin direkt durch eine reduktive Aminierung in die Dimethylaminofunktion überführt. Eine abschließende Birch-Reduktion zur Abspaltung der Benzylschutzgruppe führte in sehr guten Ausbeuten zu den freien sekundären Alkoholen $\alpha-103$ und $\beta$-103. Eine angestrebte einstufige Sequenz dieser drei Schritte war nicht erfolgreich, da die Benzylschutzgruppe unter den verwendeten Bedingungen nicht abspaltbar war.

Mit den Glykosiden $\alpha-103, \beta-103$ und dem Tricyclus 60 konnten erste Untersuchungen zur Kupplung dieser Bausteine erfolgen. Der Syntheseplan sah eine Transformation der sekundären Alkoholfunktion in ein Halogenid vor (Abbildung 52). Diese musste unter Inversion der Stereoinformation erfolgen. Es wurde eine Vielzahl von 
Bedingungen getestet, jedoch war die Einführung eines Halogenids weder unter Inversion noch unter Retention der Konfiguration möglich.

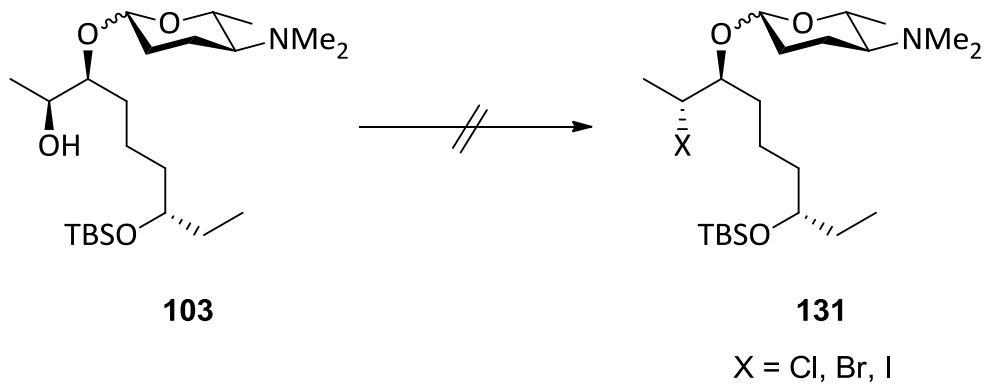

Abbildung 52: $\quad$ Synthese eines Halogenids vom Typ 131.

Eine weitere Möglichkeit zur Kupplung der Bausteine bestand darin, die Reaktivität der Aldehydfunktion in Verbindung 60 umzupolen, um einen nucleophilen Angriff auf die C-9-Kette durchzuführen. Eine Überführung des Aldehyds in ein TMS-Cyanhydrin des Typs 128 war nicht erfolgreich. Unter Verwendung der Corey-Seebach-Methode konnte jedoch das Dithian 127 hergestellt werden (Abbildung 53).

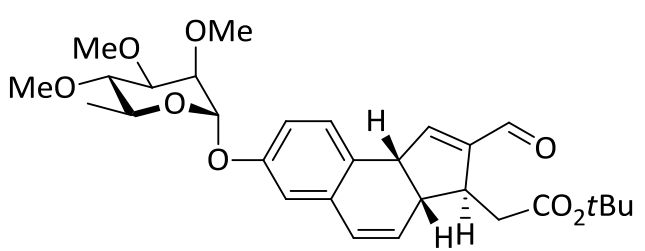

60

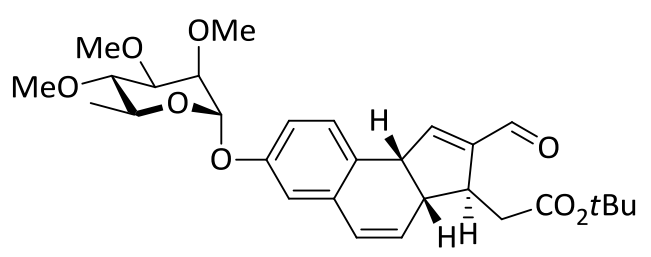

60

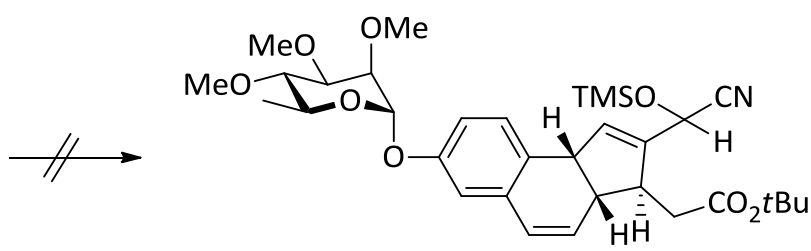

128

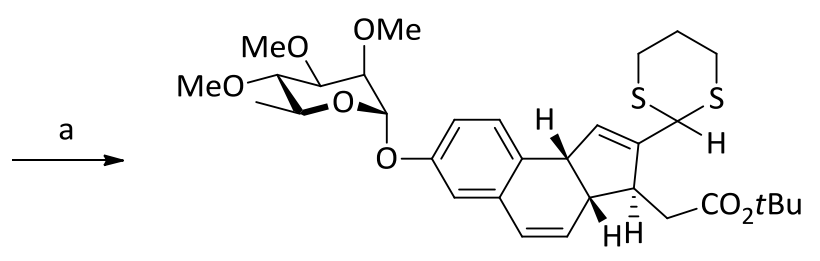

127

Abbildung 53: Synthese der Verbindungen 127 und 128: a) 1,3-Propandithiol, $\mathrm{BF}_{3} \cdot \mathrm{Et}_{2} \mathrm{O},-65^{\circ} \mathrm{C}$, dann $16 \mathrm{~h},-20^{\circ} \mathrm{C}, 2 \mathrm{~h}$.

Zur Kupplung mit dem Dithian 127, musste die sekundäre Alkoholfunktion der Glykoside $\alpha-103$ und $\beta-103$ in eine Abgangsgruppe umgewandelt werden. Eine Transformation in das entsprechende Tosylat oder Triflat war allerdings auch bei breiter Variation der Reaktionsbedingungen nicht möglich (Abbildung 54). 


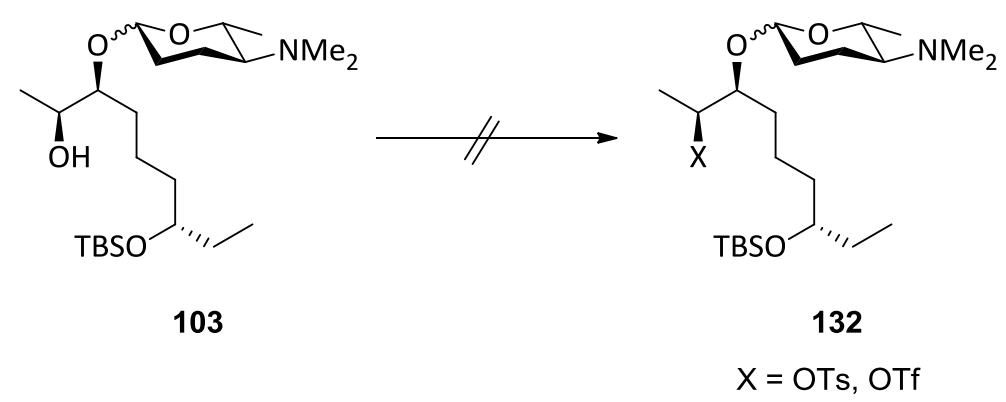

Abbildung 54: $\quad$ Synthese eines Glykosids vom Typ 132. 


\section{F. EXPERIMENTELLER TEIL}

\section{Allgemeine Arbeitstechniken}

Die Umsetzungen wurden, soweit nötig, in ausgeheizten Glasapparaturen unter einem leichten Argon-Überdruck durchgeführt. Die Lösungsmittel wurden entsprechend den üblichen Laboratoriumsmethoden getrocknet und destilliert ${ }^{78} \mathrm{bzw}$. als p.a.-Ware über Molsieb gelagert. Soweit nicht anders vermerkt, wurden kommerziell erhältliche Produkte ohne weitere Reinigung eingesetzt. Das Entgasen von Lösungsmitteln geschah durch Einleiten eines Argon-Gasstroms über einen längeren Zeitraum. Konzentrationsangaben beziehen sich, sofern nicht anders erwähnt, auf wässrige Lösungen.

\subsection{Verwendete Geräte}

Drehwerte: Drehwerte wurden mit einem Polarimeter Modell 241 der Firma PerkinElmer sowie P-2000 der Firma Jasco gemessen.

Schmelzpunkte: Die Bestimmung der Schmelzpunkte erfolgte mit Hilfe eines vollautomatischen Schmelzpunktbestimmungsgeräts Modell MPA120 (EZ-Melt) der Firma Stanford Research Systems.

Infrarotspektren: Sofern „(KBr)“ oder „(Film)“ hinter der Bezeichnung „IR“ vermerkt ist, wurden die Infrarotspektren mit dem Modell Vector 22 der Firma Bruker aufgenommen, wobei Feststoffe als KBr-Presslinge und Flüssigkeiten als Film zwischen $\mathrm{NaCl}$-Platten gemessen wurden. Findet sich kein Hinweis hinter der Bezeichnung „IR“, wurden die Infrarotspektren mit dem Modell FT/IR-4100 der Firma Jasco aufgenommen.

UV/Vis-Spektren: Die Aufnahme der UV/Vis-Spektren erfolgte mit den Modellen Lambda 2 der Firma Perkin-Elmer und V-630 der Firma Jasco. 
${ }^{1}$ H-NMR-Spektren: Die ${ }^{1}$ H-NMR-Spektren wurden mit den Modellen Mercury 300, Mercury-Vx 300, Unity 300, VNMRS 300 (jeweils 300 MHz) sowie Inova 600 (600 MHz) der Firma Varian von in deuterierten Solventien gelösten Proben aufgenommen. Die chemischen Verschiebungen sind in Einheiten der $\delta$-Skala angegeben und auf das Signal des angegebenen Lösungsmittels referenziert. Zur Kennzeichnung der Multiplizitäten der Signale wurden folgende Abkürzungen verwendet: s (Singulett), d (Dublett), t (Triplett), q (Quartett), dd (Dublett vom Dublett), dt (Dublett vom Triplett) usw. Signale, die durch Überlagerung oder Anteile höherer Ordnung nicht interpretierbar waren, wurden mit m (Multiplett) bzw. $\mathrm{m}_{\mathrm{c}}$ (symmetrisches, zentriertes Multiplett) bezeichnet und verbreiterte Signale durch den Zusatz br indiziert. Zur Bezeichnung aromatischer Protonen oder Kohlenstoffatome wurden folgende Abkürzungen verwendet: $i$ (ipso), $o$ (ortho), $m$ (meta), $p$ (para). Sofern nicht anders angegeben, beziehen sich alle Kopplungskonstanten $J$ auf ${ }^{1} \mathrm{H}-{ }^{1} \mathrm{H}-$ Kopplungen und sind in Hertz $(\mathrm{Hz})$ angegeben.

${ }^{13}$ C-NMR-Spektren: Soweit nicht anders vermerkt, wurden die ${ }^{1} \mathrm{H}$-breitbandentkoppelten ${ }^{13} \mathrm{C}$-NMR-Spektren mit den Geräten Unity $300(75 \mathrm{MHz})$, Inova $500(125 \mathrm{MHz})$ und Inova 600 (150 MHz) der Firma Varian aufgenommen. Die chemischen Verschiebungen sind in Einheiten der $\delta$-Skala angegeben. Als interner Standard diente das angegebene Lösungsmittel. Generell erfolgte die Zuordnung der Signale durch die 2DNMR-Experimente COSY, HSQC sowie HMBC.

Massenspektren: Zur Aufnahme der EI- und EI-HRMS-Spektren diente ein doppelfokussierendes Sektorfeld-Massenspektrometer MAT 95 der Firma Finnigan. Auch die DCl-Spektren mit $\mathrm{NH}_{3}$ als Reaktandgas wurden mit diesem Gerät gemessen. ESISpektren wurden mit einem Ion-Trap-Massenspektrometer LCQ der Firma Finnigan sowie einem Time-of-Flight Massenspektrometer micrOTOF der Firma Bruker aufgenommen. Die Messung der ESI-HRMS-Spektren erfolgte an einem 7-TeslaFourier-Transform-Ion-Cyclotron-Resonance (FTICR)-Massenspektrometer APEX IV der Firma Bruker und am micrOTOF-Gerät. Angegeben werden die Quotienten aus 
Masse zu Ladung sowie in Klammern die relativen Intensitäten bezogen auf den Basispeak ( $=100)$.

Kältetechnik: Die Reaktionsführung bei konstant tiefen Temperaturen über einen längeren Zeitraum erfolgte unter Einsatz von Kryostaten. Benutzt wurde das Modell EK 90 der Firma Haake. Darüber hinaus wurden übliche Kältemischungen wie Eis / Wasser- und Aceton / Trockeneis-Gemische zur temporären Kühlung verwendet.

\subsection{Chromatographische Methoden}

Dünnschichtchromatographie (DC): Es wurden Aluminium-Fertigfolien Si60 $\mathrm{F}_{254}$ der Firma Merck verwendet. Angegeben sind $\mathrm{R}_{f}$-Werte (Laufhöhe der Substanz relativ zur Höhe der Laufmittelfront). Neben der UV-Detektion diente eine Vanillin-Schwefelsäure-Lösung (0.5 g Vanillin, $3 \mathrm{~mL}$ konz. $\mathrm{H}_{2} \mathrm{SO}_{4}, 85 \mathrm{~mL} \mathrm{MeOH}$ und $10 \mathrm{~mL} \mathrm{HOAc}$ ) als Anfärbereagenz.

Säulenchromatographie: Alle säulenchromatographischen Trennungen wurden unter erhöhtem Druck (Flash-Chromatographie bei Drücken von 0.1-0.6 bar) mit Kieselgel 60 (Korngröße: 0.032-0.063 mm) der Firma Merck durchgeführt.

\section{Hochdruckflüssigkeitschromatographie (HPLC):}

Analytische HPLC: Analytische Trennungen wurden auf einer HPLC-Anlage der Firma Jasco, ausgestattet mit einer Lösungsmittelpumpe PU-2080, einer Mischkammer LG1590-04, einem Multiwellenlängendetektor MD-2010 Plus und der Steuerung LC-Net II/ADC, vorgenommen. Für die Injektion wurde ein automatischer Probenwechsler (Autosampler AS-2055) derselben Firma verwendet. Zur Bedienung, Datenerfassung und Datenauswertung wurden die Computerprogramme Borwin PDA, HSS 2000 und Borwin Chromatography der Firma Jasco eingesetzt. Für die analytischen Messungen

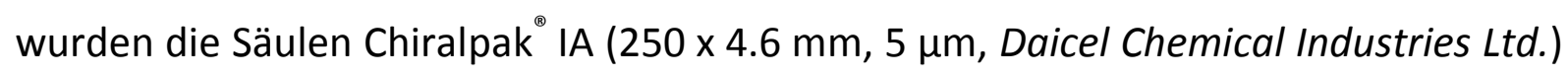
sowie Chiralpak IB (250 ${ }^{\oplus} 4.6 \mathrm{~mm}, 5 \mu \mathrm{m}$, Daicel Chemical Industries Ltd.) mit chiraler stationärer Phase verwendet. Die verwendeten Lösungsmittel waren von HPLC- 
Qualität. Alle Proben wurden membranfiltriert mit Polytetrafluorethylen-(PTFE)-Filtern der Firma Roth ( $\varnothing 25 \mathrm{~mm}, 0.2 \mu \mathrm{m})$ bzw. VWR (

Präparative HPLC: Präparative Trennungen wurden auf einem HPLC-System der Firma Jasco, ausgestattet mit zwei Lösungsmittelpumpen Modell PU-2087 PLUS und einem UV-Detektor Modell UV-2075 PLUS, vorgenommen. Verwendet wurden die Säulen Chiralpak ${ }^{\varpi}$ IA $\left(250 \times 20\right.$ mm, $5 \mu \mathrm{m}$, Daicel Chemical Industries Ltd.) sowie Chiralpak ${ }^{\circledR}$ IB (250 $\times 10$ mm, $5 \mu \mathrm{m}$, Daicel Chemical Industries Ltd.) mit chiraler stationärer Phase. Die verwendeten Lösungsmittel waren von HPLC-Qualität. Alle Proben wurden vor der Trennung membranfiltriert (siehe analytische HPLC). 


\section{Synthese der verwendeten Reagenzien}

\subsection{N,N'-Di-iso-propyl-O-tert-butyl-iso-harnstoff (87)}<smiles>CCC(C)NC(=NC(C)C)OC(C)C</smiles>

N,N'-Di-iso-propylcarbodiimid (86) (122 mL, $99.1 \mathrm{~g}, 782 \mathrm{mmol}, 1.00$ Äq.) wurde bei RT mit absolutem $t$-BuOH ( $86.1 \mathrm{~mL}, 66.7 \mathrm{~g}, 899 \mathrm{mmol}, 1.15$ Äq.) sowie $\mathrm{CuCl}$ (774 mg, $7.82 \mathrm{mmol}, 1.00 \mathrm{Mol} \%)$ versetzt. Das Reaktionsgemisch wurde für $6 \mathrm{~d}$ bei RT gerührt und im Anschluss im Vakuum (10 mbar) fraktioniert destilliert. Die Zielverbindung 87 (136 g, 680 mmol, 87\%) wurde als farbloses Öl erhalten.

Anmerkung: Verbindung 87 neigt zur Umlagerung vom Iso- in das stabilere Harnstoffderivat und ist damit selbst bei tiefer Temperatur nur begrenzt lagerfähig.

Sdp.: $64-67^{\circ} \mathrm{C}(10 \mathrm{mbar})$.

${ }^{1} \mathrm{H}-\mathrm{NMR}\left(300 \mathrm{MHz}, \mathrm{CDCl}_{3}\right): \delta(\mathrm{ppm})=1.04\left(\mathrm{dd}, J=12.2,6.3 \mathrm{~Hz}, 12 \mathrm{H}, 2 \times \mathrm{CH}\left(\mathrm{CH}_{3}\right)_{2}\right)$, 1.33-1.46 (m, $\left.9 \mathrm{H}, \mathrm{C}\left(\mathrm{CH}_{3}\right)_{3}\right), 3.11\left(\mathrm{~m}_{\mathrm{c}}, 1 \mathrm{H}, 1^{\prime}-\mathrm{H}\right), 3.22\left(\mathrm{~s}_{\mathrm{br}}, 1 \mathrm{H}, \mathrm{NH}\right), 3.48-3.78(\mathrm{~m}, 1 \mathrm{H}$, 3-H).

${ }^{13} \mathrm{C}-\mathrm{NMR}\left(126 \mathrm{MHz}, \mathrm{CDCl}_{3}\right): \delta(\mathrm{ppm})=24.0,24.4,24.7,29.2 \quad\left(2 \times \mathrm{CH}\left(\mathrm{CH}_{3}\right)_{2}\right), 28.5$ $\left(\mathrm{C}\left(\underline{C H}_{3}\right)_{3}\right), 43.6\left(\mathrm{C}-1^{\prime}\right), 46.3(\mathrm{C}-3), 78.2\left(\underline{\mathrm{C}}\left(\mathrm{CH}_{3}\right)_{3}\right), 149.5(\mathrm{C}-1)$.

$\mathrm{C}_{11} \mathrm{H}_{24} \mathrm{~N}_{2} \mathbf{O}$ (200.32).

\subsection{Polymer-gebundenes 1,8-Diazabicyclo[5.4.0]undec-7-en (93)}

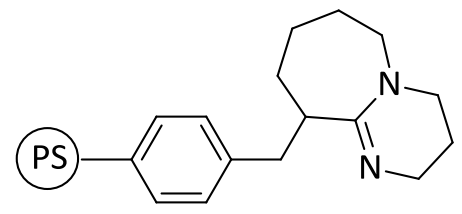

Eine Lösung von 1,8-Diazabicyclo[5.4.0]undec-7-en $(14.9 \mathrm{~mL}, 15.0 \mathrm{~g}, 100 \mathrm{mmol}$, 1.00 Äq.) in frisch destilliertem THF $(500 \mathrm{~mL})$ wurde auf $-78^{\circ} \mathrm{C}$ gekühlt und über 
einen Zeitraum von $1.5 \mathrm{~h}$ wurde $n$-Butyllithium $2.5 \mathrm{M}$ in $n$-Hexan, $38.4 \mathrm{~mL}, 96.0 \mathrm{mmol}$, 0.96 Äq.) zugetropft. Die Reaktionslösung wurde für $2 \mathrm{~h}$ bei $-78^{\circ} \mathrm{C}$ gerührt und anschließend portionsweise Merrifield-Harz (31.8 g, 2-2.5 MEQ Cl/g, 200-400 mesh, $2 \%$ crosslinked with DVB) zugegeben. Daraufhin wurde das Gemisch langsam auf RT erwärmt und für $2.5 \mathrm{~d}$ gerührt. Die Reaktion wurde durch langsame Zugabe von absolutem $\mathrm{MeOH}(10 \mathrm{~mL})$ abgebrochen, der Feststoff abfiltriert und mit absolutem THF / MeOH (1:1), MeOH, MeOH / $\mathrm{H}_{2} \mathrm{O}$ (1:1), Aceton und THF (jeweils $300 \mathrm{~mL}$ ) gewaschen. Nach $4 \mathrm{~h}$ Trocknen im Vakuum bei $70{ }^{\circ} \mathrm{C}$ wurde die Titelverbindung 93 (36.5 g) als hellgelber Feststoff erhalten.

Zur Bestimmung der Beladung wurden 200 mg des Produktes in einem Gemisch aus absolutem MeOH/1,4-Dioxan (1:2, $60 \mathrm{~mL}$ ) suspendiert und $2 \mathrm{~h}$ bei RT gerührt. Anschließende Titration mit $0.1 \mathrm{M} \mathrm{HCl}$-Lsg. unter Verwendung von Methylrot als Indikator ergab eine Beladung von $1.13 \mathrm{mmol} / \mathrm{g}$.

\section{3 lodmethyl-triphenyl-phosphonium-iodid (97)}<smiles>[O-][PbH]([O-])CI</smiles>

Triphenylphosphin (5.32 g, $20.3 \mathrm{mmol}, 1.00$ Äq.) wurde in absolutem Toluol (15 mL) vorgelegt und bei RT mit Diiodmethan (1.72 mL, $5.71 \mathrm{~g}, 21.3 \mathrm{mmol}, 1.05$ Äq.) versetzt. Das Reaktionsgemisch wurde für $14 \mathrm{~h}$ bei $80^{\circ} \mathrm{C}$ gerührt. Der ausgefallene Feststoff wurde anschließend abfiltriert und mit $n$-Pentan $(8 \times 40 \mathrm{~mL})$ gewaschen. Nach Trocknung im Hochvakuum wurde das Wittig-Salz 97 (6.50 g, $12.3 \mathrm{mmol}, 60 \%)$ in Form eines farblosen Pulvers analysenrein erhalten.

UV (MeOH): $\lambda_{\max }(\lg \varepsilon)=202 \mathrm{~nm}$ (4.8376), 221 (4.5971), 268 (3.5931), 275 (3.5093).

IR $(\mathrm{KBr}): \tilde{v}\left(\mathrm{~cm}^{-1}\right)=3041,2987,2916,2848,2742,2147,1586,1482,1438,1335,1317$.

${ }^{1} \mathrm{H}-\mathrm{NMR}\left(300 \mathrm{MHz}, \mathrm{DMSO}-\mathrm{d}_{6}\right): \delta(\mathrm{ppm})=5.03\left(\mathrm{~d}, J=8.6 \mathrm{~Hz}, 2 \mathrm{H}, \mathrm{CH}_{2}\right), 7.74-7.96(\mathrm{~m}$, $\left.15 \mathrm{H}, 3 \times \mathrm{Ph}-\mathrm{H}_{5}\right)$. 
${ }^{13}$ C-NMR (75 MHz, DMSO-d $): \delta(p p m)=118.3\left(\mathrm{~d}, J_{C, P}=88.7 \mathrm{~Hz}, 3 \times \mathrm{Ph}-\mathrm{C}_{i}\right), 130.0$ (d, $\left.J_{C, P}=12.7 \mathrm{~Hz}, 6 \times \mathrm{Ph}-\mathrm{C}_{0}\right), 133.7\left(\mathrm{~d}, J_{C, P}=10.1 \mathrm{~Hz}, 6 \times \mathrm{Ph}-\mathrm{C}_{m}\right), 135.0\left(\mathrm{~d}, J_{\mathrm{C}, \mathrm{P}}=2.9 \mathrm{~Hz}\right.$, $\left.3 \times \mathrm{Ph}-\mathrm{C}_{p}\right), 223.5\left(\mathrm{~d}, J_{\mathrm{C}, \mathrm{P}}=51.8 \mathrm{~Hz}, \mathrm{CH}_{2}\right)$.

MS $(E I, 70 \mathrm{eV}): m / z(\%)=275.4(100)[\mathrm{M}-\mathrm{I}-\mathrm{HI}]^{+}, 403.0(56)[\mathrm{M}-\mathrm{I}]^{+}, 932.4(15)[2 \mathrm{M}-\mathrm{I}]^{+}$. $\mathrm{C}_{19} \mathrm{H}_{17} \mathrm{I}_{2} \mathrm{P}$ (530.12). 


\section{Synthese des cis-disubstituierten Cyclopentenderivats 69}

\subsection{Malonsäuremonomethylester (74)}<smiles>COC(=O)CC(=O)O</smiles>

Zu einer Lösung des Malonsäuredimethylesters 73 (152 g, 1.15 mol, 1.00 Äq.) in absolutem $\mathrm{MeOH}(500 \mathrm{~mL})$ wurde $\mathrm{KOH}(64.6 \mathrm{~g}, 1.15 \mathrm{~mol}, 1.00 \mathrm{Äq}$.$) , gelöst in$ absolutem $\mathrm{MeOH}(500 \mathrm{~mL})$, getropft und das Gemisch für $24 \mathrm{~h}$ bei RT gerührt. Anschließend wurde für $1 \mathrm{~h}$ unter Rückfluss erhitzt, im Eisbad abgekühlt und das Mono-Kaliumsalz abfiltriert. Durch Einengen der Mutterlauge unter vermindertem Druck und nochmaliges Abkühlen im Eisbad konnte eine weitere Fraktion an MonoKaliumsalz erhalten werden. Die beiden Feststofffraktionen wurden vereint, mit kaltem $\mathrm{Et}_{2} \mathrm{O}(100 \mathrm{~mL})$ gewaschen und anschließend in $\mathrm{H}_{2} \mathrm{O}(300 \mathrm{~mL})$ aufgenommen. $\mathrm{Zu}$ dieser Lösung wurde unter Rühren und Eiskühlung konz. HCl-Lsg. (100 mL) getropft. Das Gemisch wurde mit $\mathrm{Et}_{2} \mathrm{O}(15 \times 150 \mathrm{~mL})$ extrahiert, die organischen Extrakte vereint, über $\mathrm{Na}_{2} \mathrm{SO}_{4}$ getrocknet und das Lösungsmittel im Vakuum entfernt. Die Zielverbindung 74 (95.1 g, 805 mmol, 70\%) wurde als farblose Flüssigkeit analysenrein erhalten.

IR (Film): $\tilde{v}\left(\mathrm{~cm}^{-1}\right)=2961,1741,1442,1335,1214,1162,1018,957,895,662$.

${ }^{1} \mathrm{H}-\mathrm{NMR}\left(300 \mathrm{MHz}, \mathrm{CDCl}_{3}\right): \delta(\mathrm{ppm})=3.41\left(\mathrm{~s}, 2 \mathrm{H}, \mathrm{CH}_{2}\right), 3.74\left(\mathrm{~s}, 3 \mathrm{H}, \mathrm{CO}_{2} \mathrm{CH}_{3}\right), 8.19\left(\mathrm{~s}_{\mathrm{br}}\right.$, $\left.1 \mathrm{H}, \mathrm{CO}_{2} \mathrm{H}\right)$.

${ }^{13} \mathrm{C}-\mathrm{NMR}\left(126 \mathrm{MHz}, \mathrm{CDCl}_{3}\right): \delta(\mathrm{ppm})=40.7\left(\mathrm{CH}_{2}\right), 52.8\left(\mathrm{CO}_{2} \underline{\mathrm{CH}}_{3}\right), 167.1\left(\underline{\mathrm{CO}}_{2} \mathrm{CH}_{3}\right)$, $171.0\left(\mathrm{CO}_{2} \mathrm{H}\right)$.

MS (DCl): $m / z(\%)=254.3(100)\left[2 \mathrm{M}+\mathrm{NH}_{4}\right]^{+}, 136.2(46)\left[\mathrm{M}+\mathrm{NH}_{4}\right]^{+}$.

$\mathrm{C}_{4} \mathrm{H}_{6} \mathbf{O}_{4}$ (118.09). 


\section{2 rac-2-Chlor-2-chlorcarbonyl-essigsäuremethylester (75)}<smiles>COC(=O)C(Cl)C(=O)Cl</smiles>

Zu der Carbonsäure 74 (44.2 g, 374 mmol, 1.00 Äq.) wurde bei RT unter heftigem Rühren zügig SOCl 2 (109 mL, 179 g, 1.50 mol, 4.01 Äq.) gegeben. Das Gemisch wurde für 30 min mit einem vorgeheizten Ölbad auf $70^{\circ} \mathrm{C}$ erwärmt, dann auf RT abgekühlt und mit $\mathrm{N}$-Chlorsuccinimid $\left(100 \mathrm{~g}, 748 \mathrm{mmol}, 2.00 \mathrm{Äq}\right.$.) sowie weiterem $\mathrm{SOCl}_{2}$ (50.0 mL, 82.0 g, 689 mmol, 1.84 Äq.) versetzt. Im Anschluss wurde die Reaktionslösung für 100 min mit einem auf $85^{\circ} \mathrm{C}$ vorgeheizten Ölbad erwärmt und die Reaktion dann durch Abkühlen auf RT abgebrochen. Überschüssiges $\mathrm{SOCl}_{2}$ wurde über eine Kühlfalle im Vakuum entfernt, der Rückstand in $\mathrm{CCl}_{4}(25 \mathrm{~mL})$ aufgenommen und filtriert. Das Filtrat wurde unter vermindertem Druck eingeengt und der Rückstand im Vakuum (8-9 mbar) fraktioniert destilliert. Das Säurechlorid 75 (48.9 g, 286 mmol, 76\%) wurde als farblose Flüssigkeit erhalten.

Sdp.: $53-55^{\circ} \mathrm{C}(8-9 \mathrm{mbar})$.

${ }^{1} \mathrm{H}-\mathrm{NMR}\left(300 \mathrm{MHz}, \mathrm{CDCl}_{3}\right): \delta(\mathrm{ppm})=3.92\left(\mathrm{~s}, 3 \mathrm{H}, \mathrm{CO}_{2} \mathrm{CH}_{3}\right), 5.18(\mathrm{~s}, 1 \mathrm{H}, 2-\mathrm{H})$.

${ }^{13} \mathrm{C}-\mathrm{NMR}\left(126 \mathrm{MHz}, \mathrm{CDCl}_{3}\right): \delta(\mathrm{ppm})=54.5\left(\mathrm{CO}_{2} \underline{\mathrm{CH}}_{3}\right), 62.3(\mathrm{C}-2), 162.9$ (C-1), 164.6 (C-3).

$\mathrm{C}_{4} \mathrm{H}_{4} \mathrm{Cl}_{2} \mathrm{O}_{3}$ (170.98).

\section{3 rac-(1R,5S)-6-Chlor-7-Oxobicyclo[3.2.0]hept-3-en-6-carbonsäureme-} thylester (78)

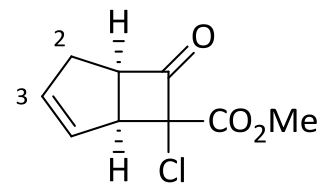

Zu einer Lösung von frisch destilliertem Carbonsäurechlorid 75 (13.2 g, 77.2 mmol, 1.00 Äq.) und Cyclopentadien (77) (25.5 g, 386 mmol, 5.00 Äq.) in absolutem $n$-Hexan 
(550 mL) wurde bei RT unter heftigem Rühren über einen Zeitraum von $4 \mathrm{~h} \mathrm{NEt}_{3}$ $(11.7 \mathrm{~mL}, 8.54 \mathrm{~g}, 84.4 \mathrm{mmol}, 1.09$ Äq.), gelöst in absolutem $n$-Hexan $(300 \mathrm{~mL})$, getropft. Nach erfolgter Zugabe wurde das Gemisch für $10 \mathrm{~h}$ bei RT gerührt und die Reaktion durch Zugabe von $\mathrm{H}_{2} \mathrm{O}(300 \mathrm{~mL})$ abgebrochen. Die organische Phase wurde abgetrennt, über $\mathrm{MgSO}_{4}$ getrocknet und das Lösungsmittel im Vakuum entfernt. Der Bicyclus 78 (10.9 g, $54.3 \mathrm{mmol}, 70 \%)$ wurde als brauner Feststoff analysenrein erhalten.

$\mathbf{R}_{f}=0.50$ (Petrolether / $\mathrm{Et}_{2} \mathrm{O} 2: 1$ ).

${ }^{1} \mathrm{H}-\mathbf{N M R}\left(300 \mathrm{MHz}, \mathrm{CDCl}_{3}\right): \delta(\mathrm{ppm})=2.47-2.61\left(\mathrm{~m}, 1 \mathrm{H}, 2-\mathrm{H}_{\mathrm{A}}\right), 2.80\left(\mathrm{~m}_{\mathrm{c}}, 1 \mathrm{H}, 2-\mathrm{H}_{\mathrm{B}}\right)$, $3.83\left(\mathrm{~s}, 3 \mathrm{H}, \mathrm{CO}_{2} \mathrm{CH}_{3}\right), 4.04-4.12(\mathrm{~m}, 1 \mathrm{H}, 1-\mathrm{H}), 4.19\left(\mathrm{~m}_{\mathrm{c}}, 1 \mathrm{H}, 5-\mathrm{H}\right), 5.73\left(\mathrm{~m}_{\mathrm{c}}, 1 \mathrm{H}, 3-\mathrm{H}\right)$, $5.98\left(m_{c}, 1 \mathrm{H}, 4-\mathrm{H}\right)$.

${ }^{13} \mathrm{C}-\mathrm{NMR}\left(126 \mathrm{MHz}, \mathrm{CDCl}_{3}\right): \delta(\mathrm{ppm})=36.0(\mathrm{C}-2), 50.5,53.9,61.2\left(\mathrm{C}-1, \mathrm{C}-5, \mathrm{CO}_{2} \mathrm{CH}_{3}\right)$, 79.8 (C-6), 127.9 (C-3), 136.1 (C-4), $166.6\left(\mathrm{CO}_{2} \mathrm{CH}_{3}\right), 199.4$ (C-7).

$\mathrm{C}_{9} \mathrm{H}_{9} \mathrm{ClO}_{3}$ (200.62).

Darstellung von Cyclopentadien:

Dicyclopentadien wurde bei Normaldruck auf $180^{\circ} \mathrm{C}$ erhitzt und das dabei im Gleichgewicht entstehende Monomer 77 (Sdp.: $\approx 40^{\circ} \mathrm{C}$ ) über eine Vigreux-Kolonne kontinuierlich in eine auf $-78{ }^{\circ} \mathrm{C}$ gekühlte Vorlage abdestilliert.

\section{4 rac-(1R,5R,6R/S)-7-Oxobicyclo[3.2.0]hept-3-en-6-carbonsäuremethyl- ester (79)}

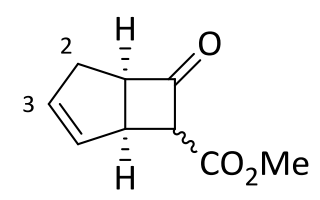

Zu einer Lösung des Bicyclus 78 (17.9 g, 89.2 mmol, 1.00 Äq.) in Eisessig (200 mL) wurde bei $0^{\circ} \mathrm{C}$ über einen Zeitraum von $20 \mathrm{~min}$ portionsweise Zinkstaub $(23.3 \mathrm{~g}$, $357 \mathrm{mmol}$, 4.00 Äq.) zugegeben. Nach Entfernen der Kühlung wurde das Gemisch 
75 min bei RT gerührt und anschließend das überschüssige Zink abfiltriert. Das Filtrat wurde mit Eiswasser $(650 \mathrm{~mL})$ verdünnt und mit $\mathrm{Et}_{2} \mathrm{O}(5 \times 200 \mathrm{~mL})$ extrahiert. Die vereinigten organischen Extrakte wurden mit ges. wässriger $\mathrm{NaHCO}_{3}$-Lsg. $(600 \mathrm{~mL})$ versetzt und anschließend festes $\mathrm{NaHCO}_{3}$ bis zur Beendigung der Gasentwicklung zugegeben. Die organische Phase wurde über $\mathrm{MgSO}_{4}$ getrocknet und das Lösungsmittel unter vermindertem Druck entfernt. Fraktionierte Destillation des Rückstands im Vakuum (0.05 mbar) lieferte die Zielverbindung 79 (11.6 g, $69.7 \mathrm{mmol}, 78 \%$ ) als farbloses Öl.

$\mathbf{R}_{f}=0.48$ (Petrolether / $\mathrm{Et}_{2} \mathrm{O} 2: 1$ ).

Sdp.: $57-60{ }^{\circ} \mathrm{C}(0.05 \mathrm{mbar})$.

${ }^{1} \mathrm{H}-\mathrm{NMR}\left(300 \mathrm{MHz}, \mathrm{CDCl}_{3}, 2: 1-G e m i s c h\right.$ der Diastereomere) $: \delta(\mathrm{ppm})=2.38-2.61 \quad(\mathrm{~m}$, $\left.1 \mathrm{H}, 2-\mathrm{H}_{\mathrm{A}}\right), 2.61-2.82\left(\mathrm{~m}, 1 \mathrm{H}, 2-\mathrm{H}_{\mathrm{B}}\right), 3.58-3.92\left(\mathrm{~m}, 5 \mathrm{H}, 1-\mathrm{H}, 5-\mathrm{H}, \mathrm{CO}_{2} \mathrm{CH}_{3}\right), 3.99-4.16$ und 4.36-4.40 (m, $1 \mathrm{H}, 6-\mathrm{H}), 5.77-5.99(\mathrm{~m}, 2 \mathrm{H}, 3-\mathrm{H}, 4-\mathrm{H})$.

${ }^{13}$ C-NMR (126 MHz, $\mathrm{CDCl}_{3}, 2: 1-G e m i s c h$ der Diastereomere): $\delta(\mathrm{ppm})=34.5$, 35.3 (C-2), 40.9, 41.3, 51.9, 52.5 (C-1, C-5), 60.2, 62.7, 67.0, 71.3 (C-6, $\mathrm{CO}_{2} \mathrm{CH}_{3}$ ), 129.7, 130.7, 133.5, 134.3 (C-3, C-4), 165.7, $167.1\left(\mathrm{CO}_{2} \mathrm{CH}_{3}\right), 203.6,203.9$ (C-7).

MS (DCl): $m / z(\%)=184.3(100)\left[\mathrm{M}+\mathrm{NH}_{4}\right]^{+}, 350.5(34)\left[2 \mathrm{M}+\mathrm{NH}_{4}\right]^{+}$.

$\mathrm{C}_{9} \mathrm{H}_{10} \mathrm{O}_{3}$ (166.17).

\section{5 rac-(4aS,7aR)-4,4a,7,7a-Tetrahydro-1H-cyclopenta[c]pyran-3-on (83)}

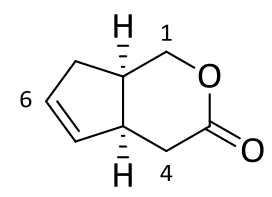

Zu einer auf $0{ }^{\circ} \mathrm{C}$ gekühlten Lösung des Bicyclus 79 (3.28 g, $19.7 \mathrm{mmol}, 1.00$ Äq.) in absolutem $\mathrm{MeOH}(100 \mathrm{~mL})$ wurde in einer Portion $\mathrm{NaBH}_{4}(2.21 \mathrm{~g}, 59.2 \mathrm{mmol}$, 3.01 Äq.) gegeben. Nach 45 min Rühren bei $0{ }^{\circ} \mathrm{C}$ wurde die Reaktion durch Entfernen des Lösungsmittels im Vakuum abgebrochen. Der Rückstand wurde in $\mathrm{Et}_{2} \mathrm{O}(60 \mathrm{~mL})$ aufgenommen und mit ges. wässriger $\mathrm{NaCl}$-Lsg. $(40 \mathrm{~mL})$ und $2 \mathrm{M} \mathrm{HCl}$-Lsg. (40 mL) ver- 
setzt. Die Phasen wurden getrennt und die wässrige Phase mit $\mathrm{Et}_{2} \mathrm{O}(4 \times 40 \mathrm{~mL})$ extrahiert. Die vereinigten organischen Phasen wurden über $\mathrm{MgSO}_{4}$ getrocknet und das Lösungsmittel im Vakuum entfernt. Der Rückstand wurde säulenchromatographisch an Kieselgel (120 g, Petrolether / $\mathrm{Et}_{2} \mathrm{O}$ 1:1) gereinigt. Die Zielverbindung 83 (2.39 g, $17.3 \mathrm{mmol}, 88 \%)$ wurde als weißer Feststoff erhalten.

$\mathbf{R}_{f}=0.22$ (Petrolether / $\mathrm{Et}_{2} \mathrm{O} 1: 1$ ).

${ }^{1} \mathrm{H}-\mathrm{NMR}\left(300 \mathrm{MHz}, \mathrm{CDCl}_{3}\right): \delta(\mathrm{ppm})=2.17-2.28\left(\mathrm{~m}, 1 \mathrm{H}, 7-\mathrm{H}_{\mathrm{A}}\right), 2.30(\mathrm{dd}, J=15.1$, $\left.7.4 \mathrm{~Hz}, 1 \mathrm{H}, 4-\mathrm{H}_{\mathrm{A}}\right), 2.58-2.83\left(\mathrm{~m}, 2 \mathrm{H}, 7-\mathrm{H}_{\mathrm{B}}, 7 \mathrm{a}-\mathrm{H}\right), 2.68\left(\mathrm{dd}, J=15.1,7.4 \mathrm{~Hz}, 1 \mathrm{H}, 4-\mathrm{H}_{\mathrm{B}}\right)$, $3.29\left(m_{c}, 1 \mathrm{H}, 4 \mathrm{a}-\mathrm{H}\right), 4.00\left(\mathrm{dd}, J=11.4,6.6 \mathrm{~Hz}, 1 \mathrm{H}, 1-\mathrm{H}_{\mathrm{A}}\right), 4.25(\mathrm{dd}, J=11.4,4.5 \mathrm{~Hz}$, $\left.1 \mathrm{H}, 1-\mathrm{H}_{\mathrm{B}}\right), 5.51\left(\mathrm{~m}_{\mathrm{c}}, 1 \mathrm{H}, 6-\mathrm{H}\right), 5.71(\mathrm{dq}, J=5.7,2.2 \mathrm{~Hz}, 1 \mathrm{H}, 5-\mathrm{H})$.

${ }^{13} \mathrm{C}-\mathrm{NMR}\left(126 \mathrm{MHz}, \mathrm{CDCl}_{3}\right): \delta(\mathrm{ppm})=33.9$ (C-7), 34.0 (C-7a), 36.2 (C-4), 42.0 (C-4a), 70.3 (C-1), 130.8, 131.8 (C-5, C-6), 173.1 (C-3).

MS (DCI): $m / z(\%)=156.2(100)\left[\mathrm{M}+\mathrm{NH}_{4}\right]^{+}, 173.2(17)\left[\mathrm{M}+\mathrm{NH}_{3}+\mathrm{NH}_{4}\right]^{+}$.

$\mathrm{C}_{8} \mathrm{H}_{10} \mathrm{O}_{2}(138.16)$

ber.: 161.0573

gef.: $161.0573[\mathrm{M}+\mathrm{Na}]^{+}$(ESI-HRMS).

\section{6 rac-(1S,5R)-2-[(5-Hydroxymethyl)-cyclopent-2-enyl]-essigsäure-tert- butylester (85)}

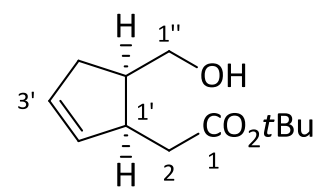

Das Lacton 83 (2.39 g, $17.3 \mathrm{mmol}, 1.00$ Äq.) wurde in absolutem $\mathrm{MeOH}(25 \mathrm{~mL})$ gelöst, mit NaOH (1.25 g, $31.1 \mathrm{mmol}, 1.80$ Äq.) versetzt und für $5 \mathrm{~h}$ unter Rückfluss erhitzt. Nach Abkühlen auf RT wurde das Lösungsmittel im Vakuum entfernt und der Rückstand $18 \mathrm{~h}$ am Hochvakuum getrocknet.

Das Salz wurde in absolutem $\mathrm{CH}_{2} \mathrm{Cl}_{2} / t$ - $\mathrm{BuOH}(80 \mathrm{~mL}, 1: 1)$ suspendiert, auf $0{ }^{\circ} \mathrm{C}$ gekühlt und mit $\mathrm{NH}_{4} \mathrm{Cl}(2.78 \mathrm{~g}, 52.0 \mathrm{mmol}, 3.01 \mathrm{Äq}$.) versetzt. Anschließend wurde frisch hergestellter Isoharnstoff $87(11.8 \mathrm{~g}, 58.7 \mathrm{mmol}, 3.39$ Äq.) tropfenweise 
zugefügt. Das Gemisch wurde für $2 \mathrm{~h}$ bei RT gerührt. Nach Abkühlen auf $0^{\circ} \mathrm{C}$ wurden erneut $\mathrm{NH}_{4} \mathrm{Cl}(1.39 \mathrm{~g}, 26.0 \mathrm{mmol}, 1.50$ Äq.) und Isoharnstoff $87(11.8 \mathrm{~g}, 58.7 \mathrm{mmol}$, 3.39 Äq.) zugefügt. Nach weiteren $2 \mathrm{~h}$ Rühren bei RT wurden wieder bei $0{ }^{\circ} \mathrm{C} \mathrm{NH}_{4} \mathrm{Cl}$ (1.39 g, $26.0 \mathrm{mmol}, 1.50$ Äq.) und Isoharnstoff 87 (11.8 g, $58.7 \mathrm{mmol}, 3.39$ Äq.) zugefügt. Nach weiteren $15 \mathrm{~h}$ Rühren bei RT wurde die Reaktion durch Zugabe von $\mathrm{H}_{2} \mathrm{O}(200 \mathrm{~mL})$ abgebrochen, die organische Phase abgetrennt und die wässrige Phase mit $\mathrm{CH}_{2} \mathrm{Cl}_{2}(5 \times 80 \mathrm{~mL})$ extrahiert. Die vereinigten organischen Extrakte wurden über $\mathrm{MgSO}_{4}$ getrocknet und im Vakuum eingeengt. Säulenchromatographische Reinigung des Rückstands an Kieselgel ( $500 \mathrm{~g}$, Petrolether / $\mathrm{Et}_{2} \mathrm{O} 2: 1$ ) lieferte das Cyclopenten 85 ( $2.90 \mathrm{~g}, 13.7 \mathrm{mmol}, 79 \%)$ als weißen Feststoff.

$\mathbf{R}_{f}=0.35$ (Petrolether / EtOAc 2:1).

$\left.{ }^{1} \mathrm{H}-\mathrm{NMR}(300 \mathrm{MHz}, \mathrm{CDCl})_{3}\right): \delta(\mathrm{ppm})=1.43\left(\mathrm{~s}, 9 \mathrm{H}, \mathrm{CO}_{2} \mathrm{C}\left(\mathrm{CH}_{3}\right)_{3}\right), 1.96-2.20(\mathrm{~m}, 2 \mathrm{H}, \mathrm{OH}$, 4'- $\left.\mathrm{H}_{\mathrm{A}}\right), 2.12\left(\mathrm{dd}, J=16.0,7.0 \mathrm{~Hz}, 1 \mathrm{H}, 2-\mathrm{H}_{\mathrm{A}}\right), 2.28-2.43\left(\mathrm{~m}, 1 \mathrm{H}, 4^{\prime}-\mathrm{H}_{\mathrm{B}}\right), 2.38(\mathrm{dd}$, $\left.J=16.0,8.0 \mathrm{~Hz}, 1 \mathrm{H}, 2-\mathrm{H}_{\mathrm{B}}\right), 2.53\left(\mathrm{~m}_{\mathrm{c}}, 1 \mathrm{H}, 5^{\prime}-\mathrm{H}\right), 3.11\left(\mathrm{~m}_{\mathrm{c}}, 1 \mathrm{H}, 1^{\prime}-\mathrm{H}\right), 3.51-3.66(\mathrm{~m}, 2 \mathrm{H}$, $\left.1^{\prime \prime}-\mathrm{H}_{2}\right), 5.69\left(\mathrm{~m}_{\mathrm{c}}, 2 \mathrm{H}, 2^{\prime}-\mathrm{H}, 3^{\prime}-\mathrm{H}\right)$.

${ }^{13} \mathrm{C}-\mathrm{NMR}\left(126 \mathrm{MHz}, \mathrm{CDCl}_{3}\right): \delta(\mathrm{ppm})=28.1\left(\mathrm{CO}_{2} \mathrm{C}\left(\mathrm{CH}_{3}\right)_{3}\right), 34.4(\mathrm{C}-4$ '), 35.5 (C-2), 42.1 (C-1'), $43.5\left(\mathrm{C}-5^{\prime}\right), 63.0\left(\mathrm{C}-1^{\prime \prime}\right), 80.7\left(\mathrm{CO}_{2} \underline{\mathrm{C}}\left(\mathrm{CH}_{3}\right)_{3}\right), 130.0\left(\mathrm{C}-3^{\prime}\right), 134.3$ (C-2'), 173.1 (C-1). MS (DCI): $m / z(\%)=174.1$ (100) $\left[\mathrm{M}+\mathrm{NH}_{4}-\text { Isobuten }\right]^{+}, 230.2$ (54) $\left[\mathrm{M}+\mathrm{NH}_{4}\right]^{+}, 213.2$ $[\mathrm{M}+\mathrm{H}]^{+}$.

$\mathrm{C}_{12} \mathrm{H}_{20} \mathrm{O}_{3}(212.29) \quad$ ber.: 235.1305

gef.: $235.1305[\mathrm{M}+\mathrm{H}]^{+}(\mathrm{ESI}-\mathrm{HRMS})$. 


\section{7 rac-(1S,5R)-2-[(5-tert-Butyldimethylsilyloxymethyl)-cyclopent-2-} enyl]essigsäure-tert-butylester (69)

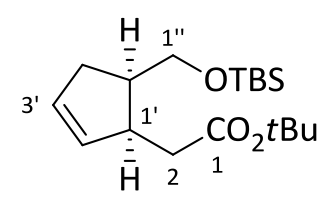

Der Alkohol 85 (1.65 g, 7.77 mmol, 1.00 Äq.) wurde in absolutem DMF (15 mL) gelöst und bei RT mit Imidazol (850 mg, 12.4 mmol, 1.60 Äq.) versetzt. Die Lösung wurde auf $0{ }^{\circ} \mathrm{C}$ abgekühlt und tropfenweise $\operatorname{TBSCl}(1.34 \mathrm{~g}, 8.94 \mathrm{mmol}, 1.15$ Äq.), gelöst in absolutem DMF (5 mL), zugefügt. Die Reaktionslösung wurde anschließend für $2 \mathrm{~h}$ bei RT gerührt. Die Reaktion wurde durch Zugabe von $\mathrm{H}_{2} \mathrm{O}(150 \mathrm{~mL})$ beendet. Die wässrige Phase wurde mit $\mathrm{Et}_{2} \mathrm{O}(3 \times 75 \mathrm{~mL})$ extrahiert und die vereinigten organischen Extrakte über $\mathrm{MgSO}_{4}$ getrocknet. Das Lösungsmittel wurde im Vakuum entfernt und der Rückstand säulenchromatographisch an Kieselgel (30 g, Petrolether / $\mathrm{Et}_{2} \mathrm{O}$ 50:1) gereinigt. Die Titelverbindung $69(2.15 \mathrm{~g}, 6.85 \mathrm{mmol}, 85 \%)$ wurde als farbloses Öl erhalten.

$\mathbf{R}_{f}=0.20$ (Petrolether / $\mathrm{Et}_{2} \mathrm{O}$ 100:1).

${ }^{1} \mathbf{H}-\mathbf{N M R}\left(300 \mathrm{MHz}, \mathrm{CDCl}_{3}\right): \delta(\mathrm{ppm})=0.02\left(\mathrm{~s}, 6 \mathrm{H}, \mathrm{Si}\left(\mathrm{CH}_{3}\right)_{2}\right), 0.86\left(\mathrm{~s}, 9 \mathrm{H}, \mathrm{SiC}\left(\mathrm{CH}_{3}\right)_{3}\right)$, $1.43\left(\mathrm{~s}, 9 \mathrm{H}, \mathrm{CO}_{2} \mathrm{C}\left(\mathrm{CH}_{3}\right)_{3}\right), 2.06\left(\mathrm{dd}, J=15.0,9.9 \mathrm{~Hz}, 1 \mathrm{H}, 2-\mathrm{H}_{\mathrm{A}}\right), 2.05-2.16(\mathrm{~m}, 1 \mathrm{H}$, 4'- $\left.\mathrm{H}_{\mathrm{A}}\right), 2.26-2.38\left(\mathrm{~m}, 1 \mathrm{H}, 4^{\prime}-\mathrm{H}_{\mathrm{B}}\right), 2.45\left(\mathrm{dd}, J=15.0,6.2 \mathrm{~Hz}, 1 \mathrm{H}, 2-\mathrm{H}_{\mathrm{B}}\right), 2.41-2.54(\mathrm{~m}$, $\left.1 \mathrm{H}, 5{ }^{\prime}-\mathrm{H}\right), 2.98-3.19\left(\mathrm{~m}, 1 \mathrm{H}, 1^{\prime}-\mathrm{H}\right), 3.52\left(\mathrm{dd}, J=10.0,7.3 \mathrm{~Hz}, 1 \mathrm{H}, 1^{\prime \prime}-\mathrm{H}_{\mathrm{A}}\right), 3.60$ (dd, $\left.J=10.0,7.1 \mathrm{~Hz}, 1 \mathrm{H}, 1^{\prime \prime}-\mathrm{H}_{\mathrm{B}}\right), 5.69\left(\mathrm{~m}_{\mathrm{c}}, 2 \mathrm{H}, 2^{\prime}-\mathrm{H}, 3^{\prime}-\mathrm{H}\right)$.

${ }^{13}$ C-NMR $\left(126 \mathrm{MHz}, \mathrm{CDCl}_{3}\right): \delta(\mathrm{ppm})=-5.3,-5.2\left(\mathrm{Si}\left(\mathrm{CH}_{3}\right)_{2}\right), 18.3 \quad\left(\mathrm{SiC}\left(\mathrm{CH}_{3}\right)_{3}\right), 26.0$ $\left(\mathrm{SiC}\left(\mathrm{CH}_{3}\right)_{3}\right), 28.2\left(\mathrm{CO}_{2} \mathrm{C}\left(\underline{\mathrm{CH}}_{3}\right)_{3}\right), 34.8\left(\mathrm{C}-4^{\prime}\right), 36.0(\mathrm{C}-2), 42.7,42.8\left(\mathrm{C}-1^{\prime}, \mathrm{C}-5^{\prime}\right), 63.2\left(\mathrm{C}-1^{\prime \prime}\right)$, $80.0\left(\mathrm{CO}_{2} \mathrm{C}\left(\mathrm{CH}_{3}\right)_{3}\right), 130.0\left(\mathrm{C}-3^{\prime}\right), 133.9$ (C-2'), 172.6 (C-1).

MS (ESI): $m / z(\%)=349.2(100)[\mathrm{M}+\mathrm{Na}]^{+}, 675.5(17)[2 \mathrm{M}+\mathrm{Na}]^{+}$.

$\mathrm{C}_{18} \mathrm{H}_{34} \mathrm{O}_{3} \mathrm{Si}(326.55) \quad$ ber.: 349.2169

gef.: $349.2169[\mathrm{M}+\mathrm{Na}]^{+}$(ESI-HRMS). 


\section{Synthese des aromatischen Fragments 62}

\subsection{Methyl- $\alpha$-L-rhamnopyranosid (89)}

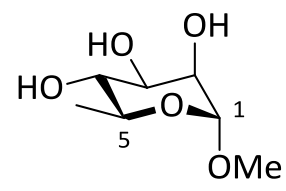

Eine Mischung von L-Rhamnose-Monohydrat (88) (9.22 g, $50.6 \mathrm{mmol}, 1.00$ Äq.) und Amberlite IR-120 (4.61 g) wurde in absolutem MeOH (185 mL) für $48 \mathrm{~h}$ unter Rückfluss erhitzt. Nach Abkühlen auf RT wurde der lonentauscher abfiltriert, mit $\mathrm{MeOH}(3 \times 50 \mathrm{~mL})$ gewaschen und das Filtrat unter vermindertem Druck eingeengt. Die Titelverbindung 89 (9.01 g, $50.6 \mathrm{mmol}$, quantitativ) wurde ohne weitere Aufreinigung analysenrein als farbloses Öl erhalten.

$\mathbf{R}_{f}=0.14\left(\mathrm{CH}_{2} \mathrm{Cl}_{2} / \mathrm{MeOH} 15: 1\right)$.

${ }^{1} \mathrm{H}-\mathrm{NMR}\left(300 \mathrm{MHz}\right.$, Aceton- $\mathrm{d}_{6}$, nach $\mathrm{D}_{2} \mathrm{O}$-Austausch): $\delta(\mathrm{ppm})=1.20 \quad(\mathrm{~d}, \quad J=6.1 \mathrm{~Hz}$, $\left.3 \mathrm{H}, 6-\mathrm{H}_{3}\right), 3.28\left(\mathrm{~s}, 3 \mathrm{H}, \mathrm{OCH}_{3}\right), 3.37(\mathrm{dd}, \mathrm{J}=9.2,4.2 \mathrm{~Hz}, 1 \mathrm{H}, 4-\mathrm{H}), 3.43-3.54(\mathrm{~m}, 1 \mathrm{H}$, 5-H), 3.54-3.63 (m, $1 \mathrm{H}, 3-\mathrm{H}), 3.76\left(\mathrm{~m}_{\mathrm{c}}, 1 \mathrm{H}, 2-\mathrm{H}\right), 4.54(\mathrm{~d}, \mathrm{~J}=1.6 \mathrm{~Hz}, 1 \mathrm{H}, 1-\mathrm{H})$.

${ }^{13}$ C-NMR (126 MHz, Aceton- $\left.\mathrm{d}_{6}\right): \delta(\mathrm{ppm})=18.1$ (C-6), $54.6\left(\mathrm{OCH}_{3}\right), 68.8(\mathrm{C}-5), 71.7$, 72.4, 73.6 (C-2, C-3, C-4), 102.0 (C-1).

MS (DCl): $m / z(\%)=196.3(100)\left[\mathrm{M}+\mathrm{NH}_{4}\right]^{+}, 374.7(51)\left[2 \mathrm{M}+\mathrm{NH}_{4}\right]^{+}$.

$\mathrm{C}_{7} \mathrm{H}_{14} \mathrm{O}_{5}$ (178.18).

\section{$4.2 \quad 1,2,3,4-$ Tetra-O-methyl- $\alpha$-L-rhamnopyranosid (90)}

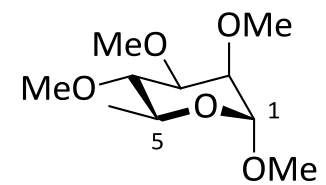

$\mathrm{NaH}$ (60\% in Mineralöl, $27.3 \mathrm{~g}, 683 \mathrm{mmol}, 13.5$ Äq.) wurde in absolutem DMSO $(100 \mathrm{~mL})$ suspendiert und bei $15{ }^{\circ} \mathrm{C}$ mit einer Lösung des Rhamnosederivats 89 (9.01 g, $50.6 \mathrm{mmol}, 1.00$ Äq.) in absolutem DMSO (300 mL) versetzt. Nach 45-minüti- 
gem Rühren bei $15^{\circ} \mathrm{C}$ wurde über einen Zeitraum von $3 \mathrm{~h}$ bei dieser Temperatur Mel (52.2 mL, 118 g, 834 mmol, 16.5 Äq.) hinzugetropft und anschließend das Reaktionsgemisch für $20 \mathrm{~h}$ bei RT gerührt. Nach Zugabe von MeOH $(25 \mathrm{~mL}$ ) wurde die Reaktionsmischung mit $\mathrm{Et}_{2} \mathrm{O}(600 \mathrm{~mL})$ versetzt, mit $\mathrm{H}_{2} \mathrm{O}(400 \mathrm{~mL})$ gewaschen und die wässrige Phase mit $\mathrm{Et}_{2} \mathrm{O}(4 \times 400 \mathrm{~mL})$ extrahiert. Die vereinigten organischen Phasen wurden mit $\mathrm{H}_{2} \mathrm{O}\left(400 \mathrm{~mL}\right.$ ) gewaschen, über $\mathrm{MgSO}_{4}$ getrocknet und das Lösungsmittel im Vakuum entfernt. Nach säulenchromatographischer Reinigung des Rückstands an Kieselgel (550 g, $n$-Pentan / EtOAc 7:1 $\rightarrow$ 3:1) wurde Verbindung 90 (5.40 g, $24.5 \mathrm{mmol}, 48 \%$ ) als hellgelbe Flüssigkeit erhalten.

$\mathbf{R}_{f}=0.24$ (n-Pentan / EtOAc 3:1).

IR (Film): $\tilde{v}\left(\mathrm{~cm}^{-1}\right)=2936,2835,1770,1448,1371,1272,1204,1109,1012,777$.

${ }^{1} \mathrm{H}-\mathrm{NMR}\left(300 \mathrm{MHz}, \mathrm{CDCl}_{3}\right): \delta(\mathrm{ppm})=1.27\left(\mathrm{~d}, J=6.3 \mathrm{~Hz}, 3 \mathrm{H}, 6-\mathrm{H}_{3}\right), 3.08(\mathrm{t}, J=9.4 \mathrm{~Hz}$, $1 \mathrm{H}, 4-\mathrm{H}), 3.32\left(\mathrm{~s}, 3 \mathrm{H}, 1-\mathrm{OCH}_{3}\right), 3.39-3.56(\mathrm{~m}, 3 \mathrm{H}, 2-\mathrm{H}, 3-\mathrm{H}, 5-\mathrm{H}), 3.45,3.46,3.52$ $\left(3 \times \mathrm{s}, 9 \mathrm{H}, 3 \times \mathrm{OCH}_{3}\right), 4.68(\mathrm{~d}, \mathrm{~J}=1.8 \mathrm{~Hz}, 1 \mathrm{H}, 1-\mathrm{H})$.

${ }^{13} \mathrm{C}-\mathrm{NMR}(126 \mathrm{MHz}, \mathrm{CDCl} 3): \delta(\mathrm{ppm})=17.8(\mathrm{C}-6), 54.8\left(1-\mathrm{OCH}_{3}\right), 57.7,59.0,60.9$ $\left(3 \times \mathrm{OCH}_{3}\right), 67.7$ (C-5), 77.3, 81.1, 82.1 (C-2, C-3, C-4), 97.8 (C-1).

MS (ESI): $m / z(\%)=243.1(100)[\mathrm{M}+\mathrm{Na}]^{+}, 463.3(18)[2 \mathrm{M}+\mathrm{Na}]^{+}$.

$\mathrm{C}_{10} \mathrm{H}_{20} \mathrm{O}_{5}(220.26) \quad$ ber.: 243.1203

gef.: $243.1205[\mathrm{M}+\mathrm{Na}]^{+}$(ESI-HRMS).

\section{$4.3 \quad 2,3,4-T r i-O-m e t h y l-\alpha / \beta-L-r h a m n o p y r a n o s i d(91)$}

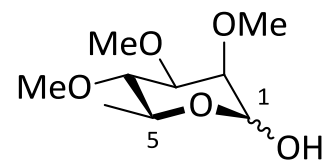

Das Rhamnosederivat 90 (5.40 g, $24.5 \mathrm{mmol}$, 1.00 Äq.) wurde in $0.5 \mathrm{M} \mathrm{H}_{2} \mathrm{SO}_{4}$-Lsg. (300 mL) gelöst und für $19 \mathrm{~h}$ bei $100{ }^{\circ} \mathrm{C}$ gerührt. Nach Abkühlen auf RT wurde die Reaktionsmischung durch Zugabe von festem $\mathrm{NaHCO}_{3}$ neutralisiert, mit festem $\mathrm{NaCl}$ gesättigt und mit EtOAc $(7 \times 100 \mathrm{~mL})$ extrahiert. Die vereinigten organischen Phasen 
wurden mit ges. wässriger $\mathrm{NaCl}$-Lsg. (200 mL) gewaschen, über $\mathrm{MgSO}_{4}$ getrocknet und das Lösungsmittel im Vakuum entfernt. Die Titelverbindung 91 (4.96 g, $24.0 \mathrm{mmol}$, 98\%) wurde nach säulenchromatographischer Reinigung an Kieselgel (150 g, $n$-Pentan / EtOAc 2:1 $\rightarrow$ 1:3) als farbloses Öl erhalten.

$\mathbf{R}_{f}=0.19$ (n-Pentan / EtOAc 1:1).

IR (Film): $\tilde{v}\left(\mathrm{~cm}^{-1}\right)=3406,2935,2830,1451,1387$, 1104, 1031, 912, 836, 789.

${ }^{1} \mathrm{H}-\mathrm{NMR}$ (300 MHz, $\mathrm{CDCl}_{3}$, 6:1-Gemisch der Anomere, $\alpha$-Anomer beschrieben): $\delta(\mathrm{ppm})=1.25\left(\mathrm{~s}, 3 \mathrm{H}, 6-\mathrm{H}_{3}\right), 3.10(\mathrm{t}, J=9.5 \mathrm{~Hz}, 1 \mathrm{H}, 4-\mathrm{H}), 3.27(\mathrm{~d}, J=3.3 \mathrm{~Hz}, 1 \mathrm{H}, \mathrm{OH})$, 3.47, $3.52\left(2 \times \mathrm{s}, 9 \mathrm{H}, 3 \times \mathrm{OCH}_{3}\right), 3.45-3.54(\mathrm{~m}, 1 \mathrm{H}, 3-\mathrm{H}), 3.57(\mathrm{dd}, J=3.5,1.8 \mathrm{~Hz}, 1 \mathrm{H}$, 2-H), 3.77 (dd, J = 9.5, 6.2 Hz, $1 \mathrm{H}, 5-\mathrm{H}), 5.22$ (dd, J = 3.3, $1.8 \mathrm{~Hz}, 1 \mathrm{H}, 1-\mathrm{H})$.

${ }^{13} \mathrm{C}-\mathrm{NMR}\left(126 \mathrm{MHz}, \mathrm{CDCl}_{3}\right.$, $\alpha$-Anomer beschrieben): $\delta(\mathrm{ppm})=17.9$ (C-6), 57.7, 59.1, $60.9\left(3 \times \mathrm{OCH}_{3}\right), 67.9$ (C-5), 77.6, 80.6, 82.1 (C-2, C-3, C-4), 91.7 (C-1).

MS (ESI): $m / z(\%)=229.1(100)[\mathrm{M}+\mathrm{Na}]^{+}, 435.2(19)[2 \mathrm{M}+\mathrm{Na}]^{+}$.

$\mathrm{C}_{9} \mathrm{H}_{18} \mathrm{O}_{5}$ (206.24) ber.: 229.1046

gef.: $229.1046[\mathrm{M}+\mathrm{Na}]^{+}$(ESI-HRMS).

\section{$4.4 \quad 0-(2,3,4-T r i-O-m e t h y l-\alpha-L-r h a m n o p y r a n o s y l)$ trichloracetimidat (92)}

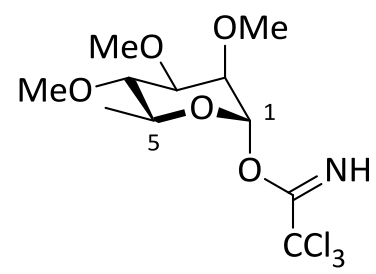

Eine Lösung von Verbindung 91 (1.00 g, $4.85 \mathrm{mmol}, 1.00$ Äq.) in absolutem $\mathrm{CH}_{2} \mathrm{Cl}_{2}$ $(90 \mathrm{~mL})$ wurde auf $0{ }^{\circ} \mathrm{C}$ gekühlt und mit Trichloracetonitril $(9.70 \mathrm{~mL}, 14.0 \mathrm{~g}$, $97.0 \mathrm{mmol}$, 20.0 Äq.) versetzt. Es wurde anschließend in mehreren Portionen Polymer-gebundenes DBU (1.26 mmol/g, 5.77 g, $7.27 \mathrm{mmol}, 1.50$ Äq.) zugegeben und 20 min bei RT gerührt. Das Reaktionsgemisch wurde über Celite filtriert und der Rückstand mit $\mathrm{CH}_{2} \mathrm{Cl}_{2}(250 \mathrm{~mL})$ gewaschen. Das Filtrat wurde im Vakuum eingeengt 
und die Titelverbindung 92 (1.63 g, 4.65 mmol, 99\%) ohne weitere Aufreingung als gelbe Flüssigkeit erhalten.

$\mathbf{R}_{f}=0.28$ (n-Pentan / EtOAc 5:1).

${ }^{1} \mathrm{H}-\mathrm{NMR}\left(300 \mathrm{MHz}, \mathrm{CDCl}_{3}\right): \delta(\mathrm{ppm})=1.31\left(\mathrm{~d}, J=6.2 \mathrm{~Hz}, 3 \mathrm{H}, 6-\mathrm{H}_{3}\right), 3.20(\mathrm{t}, J=9.5 \mathrm{~Hz}$, $1 \mathrm{H}, 4-\mathrm{H}), 3.49,3.54,3.55\left(3 \times \mathrm{s}, 9 \mathrm{H}, 3 \times \mathrm{OCH}_{3}\right), 3.48-3.57(\mathrm{~m}, 1 \mathrm{H}, 3-\mathrm{H}), 3.73$ (dd, $J=3.3,2.0 \mathrm{~Hz}, 1 \mathrm{H}, 2-\mathrm{H}), 3.75-3.80(\mathrm{~m}, 1 \mathrm{H}, 5-\mathrm{H}), 6.29(\mathrm{~d}, J=2.0 \mathrm{~Hz}, 1 \mathrm{H}, 1-\mathrm{H}), 8.57$ $\left(s_{b r}, 1 \mathrm{H}, \mathrm{NH}\right)$.

${ }^{13} \mathrm{C}-\mathrm{NMR}\left(126 \mathrm{MHz}, \mathrm{CDCl}_{3}\right): \delta(\mathrm{ppm})=17.9$ (C-6), 57.9, 59.1, $61.0\left(3 \times \mathrm{OCH}_{3}\right), 70.8$ $\left.(\mathrm{C}-5), 75.8,80.8,81.4(\mathrm{C}-2, \mathrm{C}-3, \mathrm{C}-4), 83.4\left(\mathrm{OC}(\mathrm{NH})(\underline{\mathrm{CCl}})_{3}\right)\right), 94.9(\mathrm{C}-1), 160.4$ $\left(\mathrm{O} \underline{\mathrm{C}}(\mathrm{NH})\left(\mathrm{CCl}_{3}\right)\right)$.

$\mathrm{C}_{11} \mathrm{H}_{18} \mathrm{Cl}_{3} \mathrm{NO}_{5}$ (350.62).

\subsection{2-Brom-5-methoxybenzaldehyd (95)}<smiles>COc1ccc(Br)c(C=O)c1</smiles>

3-Methoxybenzaldehyd (94) (50.0 g, $368 \mathrm{mmol}, 1.00$ Äq.) wurde in absolutem $\mathrm{CH}_{2} \mathrm{Cl}_{2}$ $(700 \mathrm{~mL})$ gelöst, die Lösung auf $0{ }^{\circ} \mathrm{C}$ gekühlt und tropfenweise mit Brom $(18.4 \mathrm{~mL}$, $58.8 \mathrm{~g}, 368 \mathrm{mmol}, 1.00$ Äq.) versetzt. Es wurde anschließend auf RT erwärmt und die Mischung für $18 \mathrm{~h}$ unter Lichtausschluss gerührt. Das Reaktionsgemisch wurde bis zur vollständigen Entfärbung mit 5\%iger wässriger $\mathrm{Na}_{2} \mathrm{~S}_{2} \mathrm{O}_{3}$-Lsg. und danach bis zur Beendigung der Gasentwicklung mit ges. wässriger $\mathrm{NaHCO}_{3}$-Lsg. versetzt. Die organische Phase wurde abgetrennt, über $\mathrm{Na}_{2} \mathrm{SO}_{4}$ getrocknet und im Vakuum eingeengt. Umkristallisation des Rohprodukts aus $n$-Pentan $(900 \mathrm{~mL}$ ) lieferte die Zielverbindung 95 (60.0 g, $279 \mathrm{mmol}, 76 \%)$ als hellgelbe Nadeln.

$\mathbf{R}_{f}=0.50$ (Petrolether / $\mathrm{Et}_{2} \mathrm{O} 4: 1$ ).

UV $\left(\mathrm{CH}_{3} \mathrm{CN}\right): \lambda_{\max }(\lg \varepsilon)=225 \mathrm{~nm}$ (4.3388), 253 (3.8498), 329 (3.4456). 
IR: $\tilde{v}\left(\mathrm{~cm}^{-1}\right)=2873,2363,1978,1895,1673,1469,1277,1197,931,864,819,647$, 597.

${ }^{1} \mathrm{H}-\mathrm{NMR}\left(300 \mathrm{MHz}, \mathrm{CDCl}_{3}\right): \delta(\mathrm{ppm})=3.81\left(\mathrm{~s}, 3 \mathrm{H}, \mathrm{OCH}_{3}\right), 7.00(\mathrm{dd}, J=8.8,3.2 \mathrm{~Hz}, 1 \mathrm{H}$, 4-H), 7.38 (d, J= 3.2 Hz, $1 \mathrm{H}, 6-\mathrm{H}), 7.49$ (d, J = 8.8 Hz, $1 \mathrm{H}, 3-\mathrm{H}), 10.28$ (s, $1 \mathrm{H}, \mathrm{CHO})$.

${ }^{13} \mathrm{C}-\mathrm{NMR}\left(126 \mathrm{MHz}, \mathrm{CDCl}_{3}\right): \delta(\mathrm{ppm})=55.7\left(\mathrm{OCH}_{3}\right), 112.6$ (C-6), $117.8(\mathrm{C}-2), 123.0$ (C-4), 133.8 (C-1), 134.4 (C-3), 159.1 (C-5), 191.5 (CHO).

MS (ESI): $m / z(\%)=516.9(100)[2 \mathrm{M}+2 \mathrm{MeOH}+\mathrm{Na}]^{+}, 269.0(63)[\mathrm{M}+\mathrm{MeOH}+\mathrm{Na}]^{+}$.

$\mathrm{C}_{8} \mathrm{H}_{7} \mathrm{BrO}_{2}$ (215.04)

ber.: 236.9522

gef.: $236.9519[\mathrm{M}+\mathrm{Na}]^{+}(\mathrm{ESI}-\mathrm{HRMS})$.

\section{6-Brom-5-hydroxybenzaldehyd (66)}

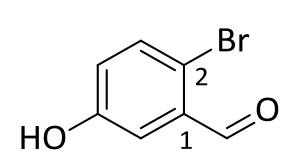

Zu einer Lösung des Aromaten 95 (5.00 g, $23.3 \mathrm{mmol}, 1.00$ Äq.) in absolutem $\mathrm{CH}_{2} \mathrm{Cl}_{2}$ (100 mL) wurde bei $-20{ }^{\circ} \mathrm{CBBr}_{3}\left(\approx 1 \mathrm{M}\right.$ in $\mathrm{CH}_{2} \mathrm{Cl}_{2}, 48.8 \mathrm{~mL}, 48.8 \mathrm{mmol}, 2.09 \mathrm{Äq}$.) langsam getropft. Die Reaktionsmischung wurde für $22 \mathrm{~h}$ bei dieser Temperatur gerührt, anschließend auf RT erwärmt und die Reaktion durch langsame Zugabe von ges. wässriger $\mathrm{NH}_{4} \mathrm{Cl}$-Lsg. $(700 \mathrm{~mL})$ beendet. Nach Zugabe von $\mathrm{Et}_{2} \mathrm{O}(500 \mathrm{~mL})$ wurde die organische Phase abgetrennt und die wässrige Phase mit $\mathrm{Et}_{2} \mathrm{O}(4 \times 30 \mathrm{~mL})$ extrahiert. Die vereinigten organischen Phasen wurden mit ges. wässriger $\mathrm{NaCl}$-Lsg. $(300 \mathrm{~mL})$ gewaschen, über $\mathrm{MgSO}_{4}$ getrocknet und das Lösungsmittel im Vakuum entfernt. Die Titelverbindung 66 (1.01 g, 4.70 mmol, 77\%) wurde nach säulenchromatographischer Reinigung des Rückstands an Kieselgel (350 g, n-Pentan / EtOAc 6:1) als hellbrauner Feststoff erhalten.

$\mathbf{R}_{f}=0.15$ (n-Pentan / EtOAc 6:1).

UV $\left(\mathrm{CH}_{3} \mathrm{CN}\right): \lambda_{\max }(\lg \varepsilon)=223 \mathrm{~nm}(4.3220), 253$ (3.8522), 330 (3.4494). 
IR: $\tilde{v}\left(\mathrm{~cm}^{-1}\right)=3314,1921,1733,1672,1590,1438,1303,1233,1169,864,831,761$, 656, 584 .

${ }^{1} \mathrm{H}-\mathrm{NMR}\left(300 \mathrm{MHz}, \mathrm{CDCl}_{3}\right): \delta(\mathrm{ppm})=5.75\left(\mathrm{~s}_{\mathrm{br}}, 1 \mathrm{H}, \mathrm{OH}\right), 7.00(\mathrm{dd}, J=8.7,3.2 \mathrm{~Hz}, 1 \mathrm{H}$, 4-H), 7.42 (d, J = 3.2 Hz, $1 \mathrm{H}, 6-\mathrm{H}), 7.50$ (d, J = 8.7 Hz, $1 \mathrm{H}, 3-\mathrm{H}), 10.27(\mathrm{~s}, 1 \mathrm{H}, \mathrm{CHO})$.

${ }^{13} \mathrm{C}-\mathrm{NMR}\left(126 \mathrm{MHz}, \mathrm{CDCl}_{3}\right): \delta(\mathrm{ppm})=115.7$ (C-6), 117.7 (C-2), 123.4 (C-4), 133.9 (C-1), 134.9 (C-3), 155.5 (C-5), 192.2 (CHO).

MS (El, $70 \mathrm{eV}): m / z(\%)=200.0(100)[\mathrm{M}]^{+}, 171.0(15)[\mathrm{M}-\mathrm{CHO}]^{+}$.

$\mathrm{C}_{7} \mathrm{H}_{5} \mathrm{BrO}_{2}$ (201.02)

ber.: 222.9365

gef.: $222.9371[\mathrm{M}+\mathrm{Na}]^{+}$(ESI-HRMS).

\subsection{2-Brom-5-(2,3,4-tri-O-methyl- $\alpha$-L-rhamnopyranosyl)benzaldehyd (96)}

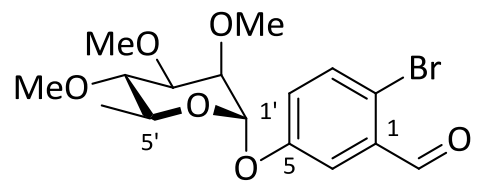

Der Aldehyd 66 (1.40 g, $6.97 \mathrm{mmol}, 1.53$ Äq.) wurde in absolutem $\mathrm{CH}_{2} \mathrm{Cl}_{2}(200 \mathrm{~mL})$ gelöst und bei RT für 90 min mit aktiviertem Molsieb $4 \AA$ (30 g) gerührt. Die Reaktionsmischung wurde auf $0{ }^{\circ} \mathrm{C}$ gekühlt und das Trichloracetimidat 92 (1.60 g, $4.56 \mathrm{mmol}, 1.00$ Äq.), gelöst in absolutem $\mathrm{CH}_{2} \mathrm{Cl}_{2}(20 \mathrm{~mL})$, sowie TMSOTf $(82.5 \mu \mathrm{L}$, $101 \mathrm{mg}, 456 \mu \mathrm{mol}, 10.0 \mathrm{Mol} \%)$, gelöst in absolutem $\mathrm{CH}_{2} \mathrm{Cl}_{2}(10 \mathrm{~mL})$, hinzugetropft. Es wurde für $75 \mathrm{~min}$ bei $0^{\circ} \mathrm{C}$ gerührt und anschließend Triethylamin $(300 \mu \mathrm{L}, 219 \mathrm{mg}$, $2.16 \mathrm{mmol}, 0.47$ Äq.) zugegeben. Nach Erwärmen auf RT wurde das Molsieb abfiltriert und mit $\mathrm{CH}_{2} \mathrm{Cl}_{2}(750 \mathrm{~mL})$ gewaschen. Das Filtrat wurde im Vakuum eingeengt und der Rückstand durch Säulenchromatographie an Kieselgel (210 g, Petrolether / MTBE 8:1 $\rightarrow$ 2:1) gereinigt. Die Titelverbindung 96 (1.56 g, $4.01 \mathrm{mmol}, 88 \%$ ) wurde als farbloses Öl erhalten.

$\mathbf{R}_{f}=0.24$ (n-Pentan / EtOAc 3:1).

${ }^{1} \mathrm{H}-\mathbf{N M R}\left(300 \mathrm{MHz}, \mathrm{CDCl}_{3}\right): \delta(\mathrm{ppm})=1.22\left(\mathrm{~d}, J=6.2 \mathrm{~Hz}, 3 \mathrm{H}, 6^{\prime}-\mathrm{H}_{3}\right), 3.17(\mathrm{t}, J=9.4 \mathrm{~Hz}$, $\left.1 \mathrm{H}, 4^{\prime}-\mathrm{H}\right), 3.54\left(\mathrm{~s}, 9 \mathrm{H}, 3 \times \mathrm{OCH}_{3}\right), 3.58-3.65\left(\mathrm{~m}, 2 \mathrm{H}, 3^{\prime}-\mathrm{H}, 5^{\prime}-\mathrm{H}\right), 3.74(\mathrm{dd}, \mathrm{J}=3.3$, 
$\left.2.0 \mathrm{~Hz}, 1 \mathrm{H}, 2^{\prime}-\mathrm{H}\right), 5.54\left(\mathrm{~d}, J=2.0 \mathrm{~Hz}, 1 \mathrm{H}, 1^{\prime}-\mathrm{H}\right), 7.16$ (dd, J= 8.8, 3.1 Hz, $\left.1 \mathrm{H}, 4-\mathrm{H}\right), 7.54$ (d, J = 8.8 Hz, $1 \mathrm{H}, 3-\mathrm{H}), 7.58(\mathrm{~d}, J=3.1 \mathrm{~Hz}, 1 \mathrm{H}, 6-\mathrm{H}), 10.27$ (s, $1 \mathrm{H}, \mathrm{CHO})$.

${ }^{13} \mathrm{C}-\mathrm{NMR}\left(126 \mathrm{MHz}, \mathrm{CDCl}_{3}\right): \delta(\mathrm{ppm})=17.9\left(\mathrm{C}^{\prime} 6^{\prime}\right), 58.0,59.3,60.9\left(3 \times \mathrm{OCH}_{3}\right), 69.0$ (C-5'), 77.0, 80.7, 81.8 (C-2', C-3', C-4'), 95.4 (C-1'), 116.9 (C-6), 119.0 (C-2), 123.6 (C-4), 134.1 (C-1), 134.6 (C-3), 155.8 (C-5), 191.2 (CHO).

MS (ESI): $m / z(\%)=443.1(100)[\mathrm{M}+\mathrm{MeOH}+\mathrm{Na}]^{+}, 411.0(51)[\mathrm{M}+\mathrm{Na}]^{+}$.

$\mathrm{C}_{16} \mathrm{H}_{21} \mathrm{BrO}_{6}$ (389.24) ber.: 411.0414

gef.: $411.0413[\mathrm{M}+\mathrm{Na}]^{+}$(ESI-HRMS).

\section{8 (Z)-2-(2-lodethenyl)-4-(2,3,4-tri-O-methyl- $\alpha$-L-rhamnopyranosyl)- brombenzol (62)}

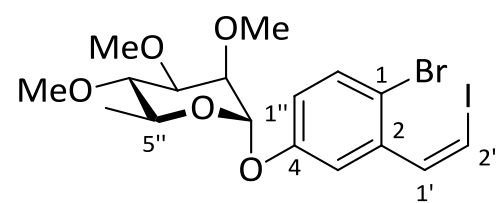

Das Wittig-Salz 97 (2.17 g, $4.08 \mathrm{mmol}, 1.50$ Äq.) wurde in absolutem THF (45 mL) suspendiert, bei RT KHMDS (0.5 M in Toluol, $9.50 \mathrm{~mL}, 4.75 \mathrm{mmol}, 1.75 \mathrm{Äq}$.) zugetropft und 5 min gerührt. Das Reaktionsgemisch wurde auf $-78^{\circ} \mathrm{C}$ gekühlt und eine Lösung des Aldehyds 96 (1.06 g, 2.72 mmol, 1.00 Äq.) in absolutem THF (25 mL) tropfenweise zugefügt. Es wurde $1 \mathrm{~h}$ bei $-78^{\circ} \mathrm{C}$ und $1.5 \mathrm{~h}$ bei RT gerührt. Die Reaktion wurde durch Zugabe von ges. wässriger $\mathrm{NH}_{4} \mathrm{Cl}$-Lsg. (200 mL) abgebrochen, die Phasen getrennt und die wässrige Phase mit Et $\mathrm{O}_{2} \mathrm{O}(4 \times 100 \mathrm{~mL})$ extrahiert. Die organischen Extrakte wurden vereint, mit ges. wässriger $\mathrm{NaCl}$-Lsg. $(150 \mathrm{~mL})$ gewaschen und über $\mathrm{MgSO}_{4}$ getrocknet. Nach Entfernung des Lösungsmittels im Vakuum wurde der Rückstand durch Säulenchromatographie an Kieselgel (350 g, Petrolether / MTBE 3:1) gereinigt. Die Titelverbindung 62 (1.09 g, $2.12 \mathrm{mmol}, 78 \%)$ konnte als hellbrauner Feststoff isoliert werden. 
$\mathbf{R}_{f}=0.34$ (Petrolether / MTBE 1:1).

${ }^{1} \mathrm{H}-\mathrm{NMR}\left(300 \mathrm{MHz}, \mathrm{CDCl}_{3}\right): \delta(\mathrm{ppm})=1.25\left(\mathrm{~d}, J=6.2 \mathrm{~Hz}, 3 \mathrm{H}, 6 "-\mathrm{H}_{3}\right), 3.18(\mathrm{t}, J=9.4 \mathrm{~Hz}$, $1 \mathrm{H}, 4$ "-H), 3.54, 3.55 (2 x s, $\left.9 \mathrm{H}, 3 \times \mathrm{OCH}_{3}\right), 3.58-3.68(\mathrm{~m}, 2 \mathrm{H}, 3$ "-H, 5"-H), 3.75 (dd, $\left.J=3.3,1.9 \mathrm{~Hz}, 1 \mathrm{H}, 2^{\prime \prime}-\mathrm{H}\right), 5.52$ (d, J=1.9 Hz, $\left.1 \mathrm{H}, 1^{\prime \prime}-\mathrm{H}\right), 6.72$ (d, J = 8.5 Hz, $\left.1 \mathrm{H}, 2^{\prime}-\mathrm{H}\right)$, $6.91(\mathrm{dd}, J=8.8,2.9 \mathrm{~Hz}, 1 \mathrm{H}, 5-\mathrm{H}), 7.27\left(\mathrm{~d}, J=8.5 \mathrm{~Hz}, 1 \mathrm{H}, 1^{\prime}-\mathrm{H}\right), 7.36(\mathrm{~d}, J=2.9 \mathrm{~Hz}, 1 \mathrm{H}$, 3-H), $7.46(\mathrm{~d}, J=8.8 \mathrm{~Hz}, 1 \mathrm{H}, 6-\mathrm{H})$.

${ }^{13}$ C-NMR (126 MHz, $\left.\mathrm{CDCl}_{3}\right): \delta(\mathrm{ppm})=18.0$ (C-6"), 58.0, 59.4, $61.0\left(3 \times \mathrm{OCH}_{3}\right), 68.8$ (C-5"), 77.2, 80.8, 81.9 (C-2", C-3", C-4"), 83.8 (C-2'), 95.5 (C-1"), 115.5 (C-1), 117.8, 117.9 (C-3, C-5), 133.3 (C-6), 138.3 (C-2), 138.6 (C-1'), 155.0 (C-4).

MS (ESI): $m / z(\%)=1049.0(100)[2 \mathrm{M}+\mathrm{Na}]^{+}, 535.0(59)[\mathrm{M}+\mathrm{Na}]^{+}$.

$\mathrm{C}_{17} \mathrm{H}_{22} \mathrm{BrIO}_{5}(513.16)$

ber.: 534.9588

gef.: $534.9582[\mathrm{M}+\mathrm{Na}]^{+}(\mathrm{ESI}-\mathrm{HRMS})$. 


\section{Synthese des Tricyclus 102}

\section{$5.1 \quad 2-\{2-[(Z)-2-(2-B r o m-5-(2,3,4-t r i-O-m e t h y l-\alpha-L-r h a m n o p y r a n o s y l)-$ phenyl)-vinyl]-5-tert-butyldimethylsilyloxymethyl-cyclopent-3-enyl\}- essigsäure-tert-butylester (98)}

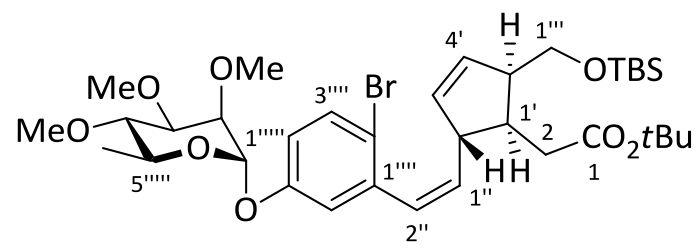

Das Vinyliodid 62 (379 mg, $739 \mu \mathrm{mol}, 1.00$ Äq.) und das Cyclopentenderivat 69 (362 mg, $1.11 \mathrm{mmol}, 1.50$ Äq.) wurden in absolutem DMF (10 mL) vorgelegt und die Lösung entgast. Danach wurden $\mathrm{Pd}(\mathrm{OAc})_{2}(16.6 \mathrm{mg}, 73.9 \mu \mathrm{mol}, 10.0 \mathrm{Mol} \%), \mathrm{NaOAc}$ (182 mg, $2.22 \mathrm{mmol}, 3.00$ Äq.) und $n-\mathrm{Bu}_{4} \mathrm{NCl}(246 \mathrm{mg}, 886 \mu \mathrm{mol}, 1.20$ Äq.) zugefügt. Die Lösung wurde für $24 \mathrm{~h}$ bei RT unter Lichtausschluss gerührt. Das Reaktionsgemisch wurde anschließend mit $\mathrm{Et}_{2} \mathrm{O}(20 \mathrm{~mL})$ verdünnt und mit $\mathrm{H}_{2} \mathrm{O}$ $(50 \mathrm{~mL})$ gewaschen. Die wässrige Phase wurde mit $\mathrm{Et}_{2} \mathrm{O}(3 \times 15 \mathrm{~mL})$ extrahiert. Die vereinigten organischen Extrakte wurden mit ges. wässriger $\mathrm{NaCl}$ Lsg. (35 mL) gewaschen, über $\mathrm{MgSO}_{4}$ getrocknet und das Lösungsmittel unter vermindertem Druck entfernt. Nach säulenchromatographischer Reinigung des Rückstands an Kieselgel (72 g, Petrolether / MTBE 7:1) wurde die Zielverbindung 98 (252 mg, $355 \mu \mathrm{mol}, 48 \%)$ als 2:1-Gemisch der Diastereomere als farbloses Öl erhalten.

$\mathbf{R}_{\boldsymbol{f}}=0.70$ (Petrolether / MTBE 1:1).

${ }^{1} \mathrm{H}-\mathrm{NMR}\left(300 \mathrm{MHz}, \mathrm{CDCl}_{3}, 2: 1-G e m i s c h\right.$ der Diastereomere): $\delta(\mathrm{ppm})=-0.11, \quad-0.09$ $\left(2 \times \mathrm{s}, 6 \mathrm{H}, \mathrm{Si}\left(\mathrm{CH}_{3}\right)_{2}\right), 0.71\left(\mathrm{~s}, 9 \mathrm{H}, \mathrm{SiC}\left(\mathrm{CH}_{3}\right)_{3}\right), 1.20$ (d, J = 6.2 Hz, $3 \mathrm{H}, 6$ "'"'- $\left.\mathrm{H}_{3}\right), 1.37$ (s, $\left.9 \mathrm{H}, \mathrm{CO}_{2} \mathrm{C}\left(\mathrm{CH}_{3}\right)_{3}\right), 2.22-2.43\left(\mathrm{~m}, 3 \mathrm{H}, 2-\mathrm{H}_{2}, 1^{\prime}-\mathrm{H}\right), 2.80\left(\mathrm{~m}_{\mathrm{c}}, 1 \mathrm{H}, 5^{\prime}-\mathrm{H}\right), 3.08-3.28(\mathrm{~m}$, 2 H, 2'-H, 4'"'-H), 3.50, 3.51, 3.52 (3 x s, 9 H, 3 x OCH $\mathrm{OCH}_{3}, 3.48-3.64$ (m, 4 H, 1"'-H , 3"'"'H, 5"'"'-H), 3.67-3.76 (m, 1 H, 2"'"-H), 5.42 (d, J=1.8 Hz, 1 H, 1"'"-H), 5.43-5.53 (m, $\left.1 \mathrm{H}, 1^{\prime}-\mathrm{H}\right), 5.59-5.79\left(\mathrm{~m}, 2 \mathrm{H}, 3^{\prime}-\mathrm{H}, 4^{\prime}-\mathrm{H}\right), 6.38$ (d, J = $\left.11.2 \mathrm{~Hz}, 1 \mathrm{H}, 2^{\prime \prime}-\mathrm{H}\right), 6.76-6.92$ (m, 2 H, 4"'--H, 6"'--H), 7.39 (d, J = 8.7 Hz, 1 H, 3"'"-H). 
${ }^{13}$ C-NMR (126 MHz, $\mathrm{CDCl}_{3}, 2: 1-G e m i s c h$ der Diastereomere): $\delta(\mathrm{ppm})=-5.6, \quad-5.4$ $\left(\mathrm{Si}\left(\mathrm{CH}_{3}\right)_{2}\right), 17.8\left(\mathrm{C}-6\right.$ '"'"), $18.0\left(\mathrm{SiC}\left(\mathrm{CH}_{3}\right)_{3}\right), 25.8\left(\mathrm{SiC}\left(\underline{\mathrm{C}} \mathrm{H}_{3}\right)_{3}\right), 28.1\left(\mathrm{CO}_{2} \mathrm{C}\left(\underline{\mathrm{C}} \mathrm{H}_{3}\right)_{3}\right), 34.5(\mathrm{C}-2)$, $44.6\left(\mathrm{C}-1^{\prime}\right), 48.6\left(\mathrm{C}-5^{\prime}\right), 49.6\left(\mathrm{C}-2^{\prime}\right), 57.9,59.2,60.8\left(3 \times \mathrm{OCH}_{3}\right), 62.5\left(\mathrm{C}-1^{\prime \prime \prime}\right), 68.6$ (C5"'"'), 77.1 (C-2"'"'), $79.9\left(\mathrm{CO}_{2} \mathrm{C}\left(\mathrm{CH}_{3}\right)_{3}\right), 80.8$ (C-3"'"'), 81.8 (C-4"'"'), 95.2 (C-1"'"'), 115.9 (C-4"'"), 116.0 (C-2"'"), 118.6 (C-6"'"), 129.5 (C-2"), 132.9 (C-3"'"), 133.6, 134.2 (C-3', C-4'), 136.3 (C-1"), 138.6 (C-1"'"), 155.1 (C-5"'"), 172.6 (C-1).

MS (ESI): $m / z(\%)=735.3(100)[\mathrm{M}+\mathrm{Na}]^{+}, 1445.6(30)[2 \mathrm{M}+\mathrm{Na}]^{+}$.

$\mathrm{C}_{35} \mathrm{H}_{55} \mathrm{BrO}_{8} \mathrm{Si}(711.80)$

ber.: 733.2742

gef.: $733.2742[\mathrm{M}+\mathrm{Na}]^{+}(\mathrm{ESI}-\mathrm{HRMS})$.

\subsection{2-[2-(tert-Butyldimethylsilyloxymethyl)-7-(2,3,4-tri-O-methyl- $\alpha$-L-} rhamnopyranosyl)-3a,9b-dihydro-3H-cyclopenta[a]naphthalin-3-yl]essigsäure-tert-butylester (100)

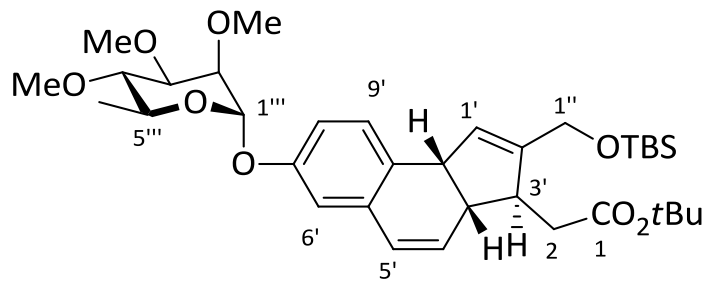

Eine Lösung von Verbindung $98(44.2 \mathrm{mg}, 62.1 \mu \mathrm{mol}, 1.00 \mathrm{Äq}$.$) und n$-Bu ${ }_{4} \mathrm{NOAc}$ (37.4 mg, $124 \mu \mathrm{mol}, 2.00$ Äq.) in einem Lösungsmittelgemisch aus DMF / $\mathrm{CH}_{3} \mathrm{CN} / \mathrm{H}_{2} \mathrm{O}$ (5:5:1, $5 \mathrm{~mL}$ ) wurde entgast und bei RT mit HBK 101 (4.08 mg, $4.34 \mu \mathrm{mol}, 6.99$ Mol\%) versetzt. Anschließend wurde das Reaktionsgemisch für $3 \mathrm{~h}$ mit einem auf $130{ }^{\circ} \mathrm{C}$ vorgeheizten Ölbad erhitzt. Nach Abkühlen auf RT wurde mit MTBE (15 mL) verdünnt und mit $\mathrm{H}_{2} \mathrm{O}(15 \mathrm{~mL})$ gewaschen. Die wässrige Phase wurde mit MTBE $(3 \times 15 \mathrm{~mL})$ extrahiert. Die vereinigten organischen Extrakte wurden über $\mathrm{MgSO}_{4}$ getrocknet und das Lösungsmittel im Vakuum entfernt. Die Titelverbindung 100 (37.6 mg, 59.6 mol, 96\%) konnte nach säulenchromatographischer Reinigung an Kieselgel (6 g, Petrolether / EtOAc 5:1) als 2:1-Gemisch der Diastereomere als farbloser Schaum isoliert werden. 
$\mathbf{R}_{f}=0.52$ (Petrolether / EtOAc 3:1).

UV (MeOH): $\lambda_{\max }(\lg \varepsilon)=228 \mathrm{~nm}$ (4.4565), 265 (3.7604), 274 (3.6873), 298 (3.4165), 309 (3.3836).

IR: $\tilde{v}\left(\mathrm{~cm}^{-1}\right)=2929,1990,1726,1602,1366,1255,1137,1100,1088,1008,992,835$, 776.

${ }^{1} \mathrm{H}-\mathrm{NMR}\left(600 \mathrm{MHz}, \mathrm{CDCl}_{3}, 2: 1-\right.$ Gemisch der Diastereomere): $\delta(\mathrm{ppm})=0.00 \quad(\mathrm{~s}, \quad 6 \mathrm{H}$, $\left.\mathrm{Si}\left(\mathrm{CH}_{3}\right)_{2}\right), 0.85\left(\mathrm{~s}, 9 \mathrm{H}, \mathrm{SiC}\left(\mathrm{CH}_{3}\right)_{3}\right), 1.23\left(\mathrm{~d}, J=6.2 \mathrm{~Hz}, 3 \mathrm{H}, 6\right.$ 6"'-H $\left.\mathrm{H}_{3}\right), 1.44(\mathrm{~s}, 9 \mathrm{H}$, $\left.\mathrm{CO}_{2} \mathrm{C}\left(\mathrm{CH}_{3}\right)_{3}\right), 2.26\left(\mathrm{dd}, J=15.4,9.9 \mathrm{~Hz}, 1 \mathrm{H}, 2-\mathrm{H}_{\mathrm{A}}\right), 2.58\left(\mathrm{dd}, J=15.4,4.3 \mathrm{~Hz}, 1 \mathrm{H}, 2-\mathrm{H}_{\mathrm{B}}\right)$, 2.94-2.99 ( $\left.m_{c}, 1 \mathrm{H}, 3^{\prime}-\mathrm{H}\right), 3.07-3.11\left(\mathrm{~m}_{\mathrm{c}}, 1 \mathrm{H}, 3 \mathrm{a}^{\prime}-\mathrm{H}\right), 3.16(\mathrm{t}, J=9.4 \mathrm{~Hz}, 1 \mathrm{H}, 4$ '"'-H), 3.52, 3.53, $3.54\left(3 \times \mathrm{s}, 9 \mathrm{H}, 3 \times \mathrm{OCH}_{3}\right), 3.61-3.67\left(\mathrm{~m}, 1 \mathrm{H}, 5^{\prime \prime}-\mathrm{H}\right), 3.64$ (dd, J = 9.4, $3.3 \mathrm{~Hz}, 1 \mathrm{H}, 3$ '"'-H), 3.71 (dd, J = 3.3, $1.7 \mathrm{~Hz}, 1 \mathrm{H}, 2$ "'-H), $4.03\left(\mathrm{~m}_{\mathrm{c}}, 1 \mathrm{H}, 9 \mathrm{~b}^{\prime}-\mathrm{H}\right), 4.17$ (d, $\left.J=1.9 \mathrm{~Hz}, 2 \mathrm{H}, 1^{\prime}-\mathrm{H}_{2}\right), 5.41\left(\mathrm{~m}_{\mathrm{c}}, 1 \mathrm{H}, 1^{\prime}-\mathrm{H}\right), 5.48(\mathrm{~d}, J=1.7 \mathrm{~Hz}, 1 \mathrm{H}, 1$ '"-H), 5.72 (dd, $\left.J=9.8,3.2 \mathrm{~Hz}, 1 \mathrm{H}, 4^{\prime}-\mathrm{H}\right), 6.20\left(\mathrm{dd}, J=9.8,2.2 \mathrm{~Hz}, 1 \mathrm{H}, 5^{\prime}-\mathrm{H}\right), 6.68(\mathrm{dd}, J=8.0,2.6 \mathrm{~Hz}$, $\left.1 \mathrm{H}, 6^{\prime}-\mathrm{H}\right), 6.82\left(\mathrm{~m}_{\mathrm{c}}, 1 \mathrm{H}, 8^{\prime}-\mathrm{H}\right), 7.01\left(\mathrm{~d}, J=8.0 \mathrm{~Hz}, 1 \mathrm{H}, 9^{\prime}-\mathrm{H}\right)$.

${ }^{13}$ C-NMR (126 MHz, CDCl $, 2: 1-G e m i s c h$ der Diastereomere): $\delta(p p m)=-5.3, \quad-5.3$ $\left(\mathrm{Si}\left(\mathrm{CH}_{3}\right)_{2}\right), 17.8(\mathrm{C}-6 " '), 18.3\left(\mathrm{SiC}\left(\mathrm{CH}_{3}\right)_{3}\right), 25.9\left(\mathrm{SiC}\left(\underline{\mathrm{CH}}_{3}\right)_{3}\right), 28.1\left(\mathrm{CO}_{2} \mathrm{C}\left(\underline{\mathrm{C}}_{3}\right)_{3}\right), 39.2(\mathrm{C}-2)$, 44.3 (C-9b'), 44.5 (C-3a'), 50.5 (C-3'), 57.8, 59.1, 60.8 (3 x OCH $\left.\mathrm{OCH}_{3}\right), 61.0$ (C-1"), 68.4 (C5"'), 77.3 (C-2"'), $80.4\left(\mathrm{CO}_{2} \mathrm{C}\left(\mathrm{CH}_{3}\right)_{3}\right), 80.8$ (C-3"'), 82.0 (C-4"'), 95.1 (C-1"'), 114.4 (C-6'), 114.8 (C-8'), 125.2 (C-5'), 128.4 (C-9a'), 128.6 (C-1'), 128.7 (C-9'), 131.9 (C-4'), 133.0 (C5a'), 144.4 (C-2'), 154.9 (C-7'), 171.8 (C-1) ppm.

MS (ESI): $m / z(\%)=653.3(100)[\mathrm{M}+\mathrm{Na}]^{+}, 1283.7(44)[2 \mathrm{M}+\mathrm{Na}]^{+}$.

$\mathrm{C}_{35} \mathrm{H}_{54} \mathrm{O}_{8} \mathrm{Si}(630.88) \quad$ ber.: 653.3480

gef.: $653.3482[\mathrm{M}+\mathrm{Na}]^{+}$(ESI-HRMS). 


\section{$5.3 \quad 2-[2-H y d r o x y m e t h y l-8-(2,3,4-t r i-0-m e t h y l-\alpha-I-r h a m n o p y r a n o s y l)-$} 3a,9b-dihydro-3H-cyclopenta[a]naphthalin-3-yl]-essigsäure-tertbutylester (102)

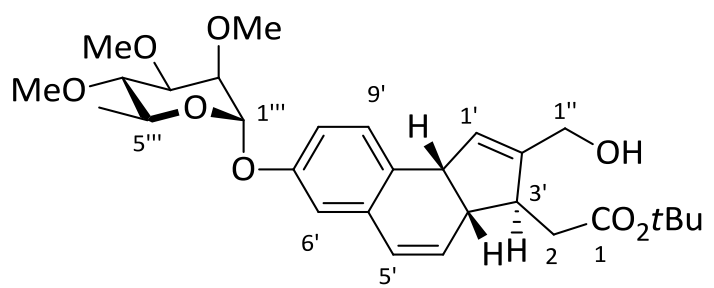

Eine Lösung des TBS-geschützten Alkohols 100 (94.6 mg, 150 mol, 1.00 Äq.) in absolutem Methanol $(15 \mathrm{~mL})$ wurde auf $0{ }^{\circ} \mathrm{C}$ gekühlt und mit $p-\mathrm{TsOH} \cdot \mathrm{H}_{2} \mathrm{O}(4.30 \mathrm{mg}$, $22.5 \mu \mathrm{mol}, 15.0 \mathrm{Mol} \%)$ versetzt. Nach $4.5 \mathrm{~h}$ Rühren bei $0{ }^{\circ} \mathrm{C}$ wurde das Reaktionsgemisch mit $\mathrm{CH}_{2} \mathrm{Cl}_{2}(15 \mathrm{~mL})$ verdünnt und mit $\mathrm{H}_{2} \mathrm{O}(20 \mathrm{~mL})$ gewaschen. Die Phasen wurden getrennt und die wässrige Phase mit $\mathrm{CH}_{2} \mathrm{Cl}_{2}(3 \times 15 \mathrm{~mL})$ extrahiert. Die vereinigten organischen Phasen wurden über $\mathrm{MgSO}_{4}$ getrocknet und das Lösungsmittel im Vakuum entfernt. Die Zielverbindung 102 (75.2 mg, 146 rmol, 97\%) wurde nach säulenchromatographischer Reinigung des Rückstands an Kieselgel (23 g, Petrolether ( MTBE 1:1) als farbloser Schaum erhalten.

$\mathbf{R}_{f}=0.35$ (Petrolether / EtOAc 1:1).

MS (ESI): $m / z(\%)=1055.6(100)[2 \mathrm{M}+\mathrm{Na}]^{+}, 539.3(43)[\mathrm{M}+\mathrm{Na}]^{+}$.

$\mathrm{C}_{29} \mathrm{H}_{40} \mathrm{O}_{8}(516.62)$

ber.: $\quad 539.2615$

gef.: $\quad 539.2618[\mathrm{M}+\mathrm{Na}]^{+}(\mathrm{ESI}-\mathrm{HRMS})$.

\subsection{Diastereomerentrennung von 2-[2-Hydroxymethyl-8-(2,3,4-tri-O- methyl- $\alpha$-L-rhamnopyranosyl)-3a,9b-dihydro-3H- cyclopenta[a]naphthalin-3-yl]-essigsäure-tert-butylester (102)}

Das Diastereomerengemisch (105 mg, $203 \mu \mathrm{mol})$ wurde in einem Gemisch aus $n$-Hexan $/ \mathrm{CH}_{2} \mathrm{Cl}_{2}(1: 1,700 \mu \mathrm{L})$ gelöst. Je $20 \mu \mathrm{L}$ dieser Probenlösung (entsprechend $3.0 \mathrm{mg}$ bzw. $5.81 \mu \mathrm{mol}$ des Gemisches) wurden in das präparative HPLC-System (Säule: Chiralpak ${ }^{\oplus} \mathrm{IB}, 250 \times 10 \mathrm{~mm}$, Partikelgröße: $5 \mu \mathrm{m}$, mobile Phase: n-Hexan / 2-Propanol = 95:5, Fluss: $6 \mathrm{~mL} / \mathrm{min}$, Druck: $4.7 \mathrm{MPa}$ ) injiziert. Fraktio- 
niertes Auffangen des Eluats (UV-Detektor: $230 \mathrm{~nm}$ ) lieferte die reinen Diastereomere (-)-102 und (+)-102.

\section{Analytische Daten für (-)-(3S,3aS, 9bS)-102:}

$\begin{array}{lll}\text { HPLC (präparativ): } & t_{\mathrm{R}}: & 9.70 \mathrm{~min} \\ & \text { Fraktion: } & 8.20-16.9 \mathrm{~min} \\ \text { HPLC (analytisch): } & \text { Säule: } & \text { Chiralpak }^{\oplus} \mathrm{IB} \\ & \text { Eluens: } & n \text {-Hexan / 2-Propanol = 93:7 } \\ & \text { Fluss: } & 0.8 \mathrm{~mL} / \mathrm{min} \\ & t_{\mathrm{R}}: & 23.68 \mathrm{~min}, 99.3 \% \text { ee. }\end{array}$

$[\alpha]_{\mathrm{D}}^{20}=-204.9^{\circ}\left(c=0.370, \mathrm{CHCl}_{3}\right)$.

UV $\left(\mathrm{CH}_{3} \mathrm{CN}\right): \lambda_{\max }(\lg \varepsilon)=227 \mathrm{~nm}$ (4.4583), 256 (3.7375), 265 (3.7754), 274 (3.7037), 299 (4.3051), 309 (3.2400).

IR: $\tilde{v}\left(\mathrm{~cm}^{-1}\right)=3447,2923,2852,2362,1722,1601,1574,1498,1365,1258,1137$, 1118, 1099, 1046, 1012, 989, 810.

${ }^{1} \mathrm{H}-\mathrm{NMR}\left(600 \mathrm{MHz}, \mathrm{CDCl}_{3}\right): \delta(\mathrm{ppm})=1.23\left(\mathrm{~d}, J=6.2 \mathrm{~Hz}, 3 \mathrm{H}, 6\right.$ '"'- $\left.\mathrm{H}_{3}\right), 1.45(\mathrm{~s}, 9 \mathrm{H}$, $\left.\mathrm{CO}_{2} \mathrm{C}\left(\mathrm{CH}_{3}\right)_{3}\right), 1.99\left(\mathrm{~s}_{\mathrm{br}}, 1 \mathrm{H}, \mathrm{OH}\right), 2.41\left(\mathrm{dd}, J=15.6,8.0 \mathrm{~Hz}, 1 \mathrm{H}, 2-\mathrm{H}_{\mathrm{A}}\right), 2.54(\mathrm{dd}, J=15.6$, $\left.5.8 \mathrm{~Hz}, 1 \mathrm{H}, 2-\mathrm{H}_{\mathrm{B}}\right), 3.02\left(\mathrm{~m}_{\mathrm{c}}, 1 \mathrm{H}, 3^{\prime}-\mathrm{H}\right), 3.08\left(\mathrm{~m}_{\mathrm{c}}, 1 \mathrm{H}, 3 \mathrm{a}^{\prime}-\mathrm{H}\right), 3.16(\mathrm{t}, J=9.4 \mathrm{~Hz}, 1 \mathrm{H}$, 4"'-H), 3.51, 3.53, $3.54\left(3 \times \mathrm{s}, 9 \mathrm{H}, 3 \times \mathrm{OCH}_{3}\right), 3.60-3.66(\mathrm{~m}, 1 \mathrm{H}, 5$ "'-H), 3.64 (dd, $\left.J=9.4,3.3 \mathrm{~Hz}, 1 \mathrm{H}, 3^{\prime \prime \prime}-\mathrm{H}\right), 3.71\left(\mathrm{dd}, J=3.3,1.9 \mathrm{~Hz}, 1 \mathrm{H}, 2^{\prime \prime}-\mathrm{H}\right), 4.04\left(m_{c}, 1 \mathrm{H}, 9 b^{\prime}-\mathrm{H}\right)$, 4.15 (s, $\left.2 \mathrm{H}, 1^{\prime \prime}-\mathrm{H}_{2}\right), 5.47$ (d, J = 1.9 Hz, $\left.1 \mathrm{H}, 1^{\prime \prime}-\mathrm{H}\right), 5.52\left(\mathrm{~m}_{\mathrm{c}}, 1 \mathrm{H}, 1^{\prime}-\mathrm{H}\right), 5.76$ (dd, J = 9.8, $\left.3.4 \mathrm{~Hz}, 1 \mathrm{H}, 4^{\prime}-\mathrm{H}\right), 6.23$ (dd, J = 9.8, 2.0 Hz, $1 \mathrm{H}, 5^{\prime}-\mathrm{H}$ ), 6.69 (d, J = 2.6 Hz, $\left.1 \mathrm{H}, 6^{\prime}-\mathrm{H}\right), 6.83$ (dd, $J=8.3,2.6 \mathrm{~Hz}, 1 \mathrm{H}, 8^{\prime}-\mathrm{H}$ ), 7.00 (d, J= $8.3 \mathrm{~Hz}, 1 \mathrm{H}, 9^{\prime}-\mathrm{H}$ ).

${ }^{13} \mathrm{C}-\mathrm{NMR}\left(126 \mathrm{MHz}, \mathrm{CDCl}_{3}\right): \delta(\mathrm{ppm})=17.9(\mathrm{C}-6 " \mathrm{\prime \prime}), 28.1\left(\mathrm{CO}_{2} \mathrm{C}\left(\mathrm{CH}_{3}\right)_{3}\right), 39.3(\mathrm{C}-2), 44.3$ (C-9b'), 44.7 (C-3a'), 50.4 (C-3'), 57.9, 59.2, 60.9 (3 x OCH 3 ), 60.6 (C-1"), 68.5 (C-5"'), 77.3 (C-2"'), 80.9 (C-3'"'), 80.9 ( $\left.\mathrm{CO}_{2} \underline{\mathrm{C}}\left(\mathrm{CH}_{3}\right)_{3}\right), 82.0$ (C-4"'), 95.1 (C-1"'), 114.6 (C-6'), 115.1 (C-8'), 125.4 (C-5'), 128.1 (C-9a'), 128.7 (C-9'), 130.2 (C-1'), 131.4 (C-4'), 132.9 (C-5a'), 144.8 (C-2'), 155.0 (C-7'), 172.2 (C-1). 
Analytische Daten für $(+)-(3 R, 3 \mathrm{a} R, 9 \mathrm{~b} R)-102:$

$\begin{array}{lll}\text { HPLC (präparativ): } & t_{\mathrm{R}}: & 3.44 \mathrm{~min} \\ & \text { Fraktion: } & 3.10-5.30 \mathrm{~min} \\ \text { HPLC (analytisch): } & \text { Säule: } & \text { Chiralpak }{ }^{\circledR} \mathrm{IB} \\ & \text { Eluens: } & n \text {-Hexan } / 2 \text {-Propanol = 93:7 } \\ & \text { Fluss: } & 0.8 \mathrm{~mL} / \mathrm{min} \\ & t_{\mathrm{R}}: & 13.68 \mathrm{~min}, 99.1 \% \text { ee. } \\ {[\alpha]_{\mathrm{D}}^{\mathbf{2 0}}=+88.2^{\circ}\left(c=0.335, \mathrm{CHCl}_{3}\right) .} & \end{array}$

5.5 (3S,3aS,9bS)-2-(2-Formyl-7-(2,3,4-tri-O-methyl- $\alpha$-L-rhamnopyranosyl)3a,9b-dihydro-3H-cyclopenta[a]naphthalin-3-yl)-essigsäure-tert-butylester (60)

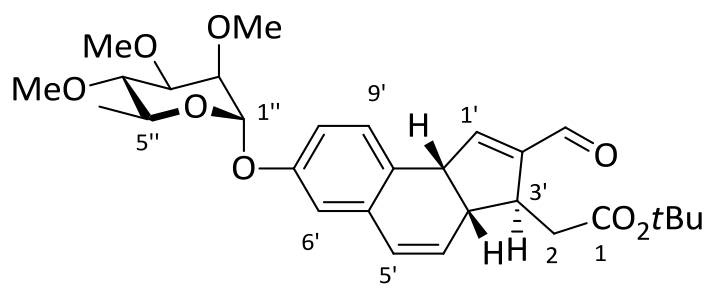

Der Alkohol 102 (20.0 mg, $38.7 \mu \mathrm{mol}, 1.00$ Äq.) wurde in absolutem $\mathrm{CH}_{2} \mathrm{Cl}_{2}$ (7.5 mL) gelöst und bei $0{ }^{\circ} \mathrm{C}$ mit DMP (28.7 mg, $67.7 \mu \mathrm{mol}, 1.75$ Äq.) versetzt. Das Reaktionsgemisch wurde $1.5 \mathrm{~h}$ bei $0^{\circ} \mathrm{C}$ gerührt und die Reaktion anschließend durch Zugabe von $1 \mathrm{M}$ wässriger $\mathrm{Na}_{2} \mathrm{~S}_{2} \mathrm{O}_{3}$-Lsg. $(4 \mathrm{~mL})$ und ges. wässriger $\mathrm{NaHCO}_{3}$-Lsg. $(4 \mathrm{~mL})$ abgebrochen. Das zunächst trübe Gemisch wurde bis zum Erhalt einer annähernd klaren, heterogenen Lösung (ca. $10 \mathrm{~min}$ ) bei $0{ }^{\circ} \mathrm{C}$ gerührt. Die Phasen wurden getrennt und die wässrige Phase mit $\mathrm{CH}_{2} \mathrm{Cl}_{2}(3 \times 7.5 \mathrm{~mL})$ extrahiert. Die vereinigten organischen Extrakte wurden über $\mathrm{MgSO}_{4}$ getrocknet und das Lösungsmittel im Vakuum entfernt. Säulenchromatographische Reinigung des Rückstands an Kieselgel (5 g, Petrolether / MTBE 1:1) lieferte das Produkt 60 (17.8 mg, $34.6 \mathrm{mmol}, 89 \%)$ als gelblichen Schaum. 
$\mathbf{R}_{f}=0.28$ (Petrolether / EtOAc 2:1).

$[\alpha]_{\mathrm{D}}^{20}=-158.0^{\circ}\left(c=0.865, \mathrm{CHCl}_{3}\right)$.

UV $\left(\mathrm{CH}_{3} \mathrm{CN}\right): \lambda_{\max }(\lg \varepsilon)=232 \mathrm{~nm}$ (4.5319), 309 (3.3689), 335 (3.2892), 354 (3.2729).

IR: $\tilde{v}\left(\mathrm{~cm}^{-1}\right)=2976,2930,2825,1724,1677,1498,1366,1259,1138,1119,1100$, 1046, 1010, 988, 841, 812, 793.

${ }^{1} \mathrm{H}-\mathrm{NMR}\left(600 \mathrm{MHz}, \mathrm{CDCl}_{3}\right): \delta(\mathrm{ppm})=1.23\left(\mathrm{~d}, J=6.2 \mathrm{~Hz}, 3 \mathrm{H}, 6 "-\mathrm{H}_{3}\right), 1.44(\mathrm{~s}, 9 \mathrm{H}$, $\left.\mathrm{CO}_{2} \mathrm{C}\left(\mathrm{CH}_{3}\right)_{3}\right), 2.27\left(\mathrm{dd}, J=15.9,10.7 \mathrm{~Hz}, 1 \mathrm{H}, 2-\mathrm{H}_{\mathrm{A}}\right), 2.77(\mathrm{dd}, J=15.9,3.6 \mathrm{~Hz}, 1 \mathrm{H}$, 2- $\left.\mathrm{H}_{\mathrm{B}}\right), 3.17\left(\mathrm{t}, J=9.4 \mathrm{~Hz}, 1 \mathrm{H}, 4^{\prime \prime}-\mathrm{H}\right), 3.24\left(\mathrm{~m}_{\mathrm{c}}, 1 \mathrm{H}, 3 \mathrm{a}^{\prime}-\mathrm{H}\right), 3.33\left(\mathrm{~m}_{\mathrm{c}}, 1 \mathrm{H}, 3^{\prime}-\mathrm{H}\right), 3.52$, 3.54, $3.54\left(3 \times \mathrm{s}, 9 \mathrm{H}, 3 \times \mathrm{OCH}_{3}\right), 3.59-3.64(\mathrm{~m}, 1 \mathrm{H}, 5 "-\mathrm{H}), 3.64(\mathrm{dd}, \mathrm{J}=9.4,3.3 \mathrm{~Hz}, 1 \mathrm{H}$, 3"-H), 3.72 (dd, J = 3.1, $2.1 \mathrm{~Hz}, 1 \mathrm{H}, 2 "-\mathrm{H}), 4.29\left(\mathrm{~m}_{\mathrm{c}}, 1 \mathrm{H}, \mathrm{b}^{\prime}-\mathrm{H}\right), 5.49$ (d, J = 1.8 Hz, $1 \mathrm{H}$, 1"-H), 5.74 (dd, J = 9.8, 3.0 Hz, $\left.1 \mathrm{H}, 4^{\prime}-\mathrm{H}\right), 6.23$ (dd, J = 9.8, 2.4 Hz, $\left.1 \mathrm{H}, 5^{\prime}-\mathrm{H}\right), 6.59\left(\mathrm{~m}_{\mathrm{c}}\right.$ $1 \mathrm{H}, 1^{\prime}-\mathrm{H}$ ), $6.72\left(\mathrm{~d}, J=2.6 \mathrm{~Hz}, 1 \mathrm{H}, 6^{\prime}-\mathrm{H}\right), 6.87$ (dd, $\left.J=8.3,2.6 \mathrm{~Hz}, 1 \mathrm{H}, 8^{\prime}-\mathrm{H}\right), 7.08$ (d, $\left.J=8.3 \mathrm{~Hz}, 1 \mathrm{H}, 9^{\prime}-\mathrm{H}\right), 9.72(\mathrm{~s}, 1 \mathrm{H}, \mathrm{CHO})$.

${ }^{13} \mathrm{C}-\mathrm{NMR}\left(126 \mathrm{MHz}, \mathrm{CDCl}_{3}\right): \delta(\mathrm{ppm})=17.9(\mathrm{C}-6 "), 28.2\left(\mathrm{CO}_{2} \mathrm{C}\left(\mathrm{CH}_{3}\right)_{3}\right), 38.4(\mathrm{C}-2), 44.1$ (C-3a'), 45.9 (C-9b'), 47.4 (C-3'), 57.9, 59.2, 60.9 (3 x OCH $)$, 68.6 (C-5"), 77.3 (C-2"), $80.7\left(\mathrm{CO}_{2} \underline{\mathrm{C}}\left(\mathrm{CH}_{3}\right)_{3}\right), 80.8$ (C-3"), 82.0 (C-4"), 95.2 (C-1"), 114.8 (C-6'), 115.3 (C-8'), 125.1 (C-9a'), 125.4 (C-5'), 128.8 (C-9'), 131.3 (C-4'), 133.4 (C-5a'), 146.4 (C-2'), 155.2 (C-1'), 155.6 (C-7'), 171.1 (C-1), 189.1 (CHO).

MS (ESI): $m / z(\%)=1051.5(100)[2 \mathrm{M}+\mathrm{Na}]^{+}, 537.2(57)[\mathrm{M}+\mathrm{Na}]^{+}$.

$\mathrm{C}_{29} \mathrm{H}_{38} \mathrm{O}_{8}$ (514.61) ber.: 537.2459

gef.: $537.2458[\mathrm{M}+\mathrm{Na}]^{+}(\mathrm{ESI}-\mathrm{HRMS})$. 


\section{Synthese des C-9 Fragments 103}

\section{1 (S)-2-(Benzyloxy)propansäuremethylester (105)}<smiles>COC(=O)C(C)Cc1ccccc1</smiles>

Methyl-(S)-(-)-lactat (104) (4.76 mL, 5.20 g, 49.9 mmol, 1.00 Äq.) und Benzyl-2,2,2-trichloracetimidat (108) $(11.2 \mathrm{~mL}, 15.2 \mathrm{~g}, 60.2 \mathrm{mmol}, 1.21$ Äq.) wurden in einem Gemisch aus absolutem Cyclohexan $/ \mathrm{CH}_{2} \mathrm{Cl}_{2}(2: 1,75 \mathrm{~mL})$ gelöst und die Lösung auf $0{ }^{\circ} \mathrm{C}$ gekühlt. Das Reaktionsgemisch wurde tropfenweise mit TfOH $(351 \mu \mathrm{L}, 595 \mathrm{mg}$, $3.96 \mathrm{mmol}$, 7.94 Mol\%) versetzt und anschließend für $8 \mathrm{~h}$ bei RT gerührt. Die Reaktion wurde durch Zugabe von ges. wässriger $\mathrm{NaHCO}_{3}$-Lsg. $(75 \mathrm{~mL}$ ) beendet und die Phasen getrennt. Die organische Phase wurde filtriert und der Rückstand mit $n$-Hexan $(100 \mathrm{~mL})$ gewaschen. Die wässrige Phase wurde mit $\mathrm{CH}_{2} \mathrm{Cl}_{2}(3 \times 30 \mathrm{~mL})$ extrahiert. Die vereinigten organischen Phasen wurden über $\mathrm{MgSO}_{4}$ getrocknet und das Lösungsmittel im Vakuum entfernt. Nach säulenchromatographischer Reinigung des Rückstands an Kieselgel (650 g, Petrolether / MTBE 20:1) wurde die Zielverbindung 105 $(8.08 \mathrm{~g}, 41.6 \mathrm{mmol}, 83 \%)$ als farblose Flüssigkeit erhalten.

$\mathbf{R}_{f}=0.35$ (Petrolether / MTBE 10:1).

${ }^{1} \mathrm{H}-\mathrm{NMR}\left(300 \mathrm{MHz}, \mathrm{CDCl}_{3}\right): \delta(\mathrm{ppm})=1.42\left(\mathrm{~d}, J=6.9 \mathrm{~Hz}, 3 \mathrm{H}, 3-\mathrm{H}_{3}\right), 3.74(\mathrm{~s}, 3 \mathrm{H}$, $\left.\mathrm{CO}_{2} \mathrm{CH}_{3}\right), 4.06(\mathrm{q}, J=6.9 \mathrm{~Hz}, 1 \mathrm{H}, 2-\mathrm{H}), 4.44\left(\mathrm{~d}, J=11.7 \mathrm{~Hz}, 1 \mathrm{H}, \mathrm{OCH}_{\mathrm{A}} \mathrm{Ph}\right), 4.68(\mathrm{~d}$, $\left.J=11.7 \mathrm{~Hz}, 1 \mathrm{H}, \mathrm{OCH}_{\mathrm{B}} \mathrm{Ph}\right), 7.21-7.41\left(\mathrm{~m}, 5 \mathrm{H}, \mathrm{Ph}-\mathrm{H}_{5}\right)$.

${ }^{13} \mathrm{C}-\mathrm{NMR}\left(126 \mathrm{MHz}, \mathrm{CDCl}_{3}\right): \delta(\mathrm{ppm})=18.7(\mathrm{C}-3), 51.9\left(\mathrm{CO}_{2} \mathrm{CH}_{3}\right), 72.0\left(\mathrm{OCH}_{2} \mathrm{Ph}\right), 73.9$ (C-2), $127.7\left(\mathrm{Ph}^{-\mathrm{C}_{p}}\right), 127.8\left(2 \times \mathrm{Ph} \mathrm{C}_{m}\right), 128.3\left(2 \times \mathrm{Ph} \mathrm{C}_{o}\right), 137.4\left(\mathrm{Ph}-\mathrm{C}_{i}\right), 173.5(\mathrm{C}-1)$. MS (ESI): $m / z(\%)=411.2(100)[2 \mathrm{M}+\mathrm{Na}]^{+}, 217.1(51)[\mathrm{M}+\mathrm{Na}]^{+}$. $\mathrm{C}_{11} \mathrm{H}_{14} \mathrm{O}_{3}$ (194.23) ber.: 217.0835 gef.: $217.0836[\mathrm{M}+\mathrm{Na}]^{+}$(ESI-HRMS). 


\section{2 (S)-2-(Benzyloxy)propanal (106)}<smiles>CC(O)C=O</smiles>

Zu einer auf $-78^{\circ} \mathrm{C}$ gekühlten Lösung des Lactats 105 (5.00 g, $25.7 \mathrm{mmol}, 1.00$ Äq.) in absolutem $\mathrm{Et}_{2} \mathrm{O}(100 \mathrm{~mL})$ wurde über einen Zeitraum von $45 \mathrm{~min}$ DIBAL $(1.0 \mathrm{M}$ in $n$-Hexan, $33.5 \mathrm{~mL}, 33.5 \mathrm{mmol}, 1.30$ Äq.), das auf $-78{ }^{\circ} \mathrm{C}$ vorgekühlt wurde, getropft. Anschließend wurde die Reaktionslösung für $20 \mathrm{~min}$ bei $-78^{\circ} \mathrm{C}$ gerührt und die Reaktion durch Zugabe von $\mathrm{H}_{2} \mathrm{O} / \mathrm{MeOH}(3: 1,48 \mathrm{~mL})$ bei dieser Temperatur abgebrochen. Es wurde langsam auf RT erwärmt, die Phasen getrennt und die organische Phase filtriert. Der Rückstand wurde mit MTBE (300 mL) gewaschen und die wässrige Phase mit MTBE $(3 \times 10 \mathrm{~mL})$ extrahiert. Die vereinigten organischen Phasen wurden über $\mathrm{MgSO}_{4}$ getrocknet und das Lösungsmittel unter vermindertem Druck entfernt. Der Rückstand wurde säulenchromatographisch an Kieselgel (360 g, Petrolether / MTBE 10:1) gereinigt und die Titelverbindung 106 (3.87 g, 23.6 mmol, 92\%) als farblose Flüssigkeit erhalten.

$\mathbf{R}_{f}=0.23$ (Petrolether / MTBE 10:1).

${ }^{1} \mathbf{H}-\mathbf{N M R}\left(300 \mathrm{MHz}, \mathrm{CDCl}_{3}\right): \delta(\mathrm{ppm})=1.32\left(\mathrm{~d}, J=6.9 \mathrm{~Hz}, 6 \mathrm{H}, 3-\mathrm{H}_{3}\right), 3.88(\mathrm{dq}, J=6.9$, $1.8 \mathrm{~Hz}, 1 \mathrm{H}, 2-\mathrm{H}), 4.58\left(\mathrm{~d}, J=11.7 \mathrm{~Hz}, 1 \mathrm{H}, \mathrm{OCH}_{\mathrm{A}} \mathrm{Ph}\right), 4.64\left(\mathrm{~d}, J=11.7 \mathrm{~Hz}, 1 \mathrm{H}, \mathrm{OCH}_{\mathrm{B}} P h\right)$, 7.27-7.39 (m, $\left.5 \mathrm{H}, \mathrm{Ph}-\mathrm{H}_{5}\right), 9.65(\mathrm{~d}, \mathrm{~J}=1.8 \mathrm{~Hz}, 1 \mathrm{H}, \mathrm{CHO})$.

${ }^{13} \mathrm{C}-\mathrm{NMR}\left(126 \mathrm{MHz}, \mathrm{CDCl}_{3}\right): \delta(\mathrm{ppm})=15.4$ (C-3), $72.0\left(\mathrm{OCH}_{2} \mathrm{Ph}\right), 79.4(\mathrm{C}-2), 127.8$ $\left(2 \times \mathrm{Ph}^{-\mathrm{C}_{o}}\right), 128.0\left(\mathrm{Ph}-\mathrm{C}_{p}\right), 128.4\left(2 \times \mathrm{Ph}-\mathrm{C}_{m}\right), 137.2\left(\mathrm{Ph}-\mathrm{C}_{i}\right), 203.2(\mathrm{CHO})$.

MS (ESI): $m / z(\%)=179.1(100)[\mathrm{M}+\mathrm{Cl}]^{-}, 163.1(25)\left[\mathrm{M}-\mathrm{H}^{+}\right]^{-}$.

$\mathrm{C}_{10} \mathrm{H}_{12} \mathrm{O}_{2}(164.20)$

ber.: 163.0765

gef.: $163.0766\left[\mathrm{M}-\mathrm{H}^{+}\right]^{-}$(ESI-HRMS). 


\section{3 (2S,3S)-2-(Benzyloxy)hex-5-en-3-ol (107)}<smiles>C=CCC(O)C(C)OCc1ccccc1</smiles>

Zu einer Lösung von $\mathrm{SnCl}_{4}\left(1.0 \mathrm{M}\right.$ in $\mathrm{CH}_{2} \mathrm{Cl}_{2}, 2.50 \mathrm{~mL}, 2.50 \mathrm{mmol}, 1.00 \mathrm{Äq}$.) in absolutem $\mathrm{CH}_{2} \mathrm{Cl}_{2}(10 \mathrm{~mL})$ wurde bei $-78^{\circ} \mathrm{C}$ über einen Zeitraum von 5 min der Aldehyd 106 (411 mg, $2.50 \mathrm{mmol}, 1.00$ Äq.), gelöst in absolutem $\mathrm{CH}_{2} \mathrm{Cl}_{2}(2.5 \mathrm{~mL})$, getropft und das Gemisch für 5 min bei dieser Temperatur gerührt. Anschließend wurde die Reaktionslösung tropfenweise mit Allyltrimethylsilan (436 $\mu \mathrm{L}, 314 \mathrm{mg}, 2.75 \mathrm{mmol}, 1.10$ Äq.) versetzt und weitere $20 \mathrm{~min}$ bei $-78{ }^{\circ} \mathrm{C}$ gerührt. Die Reaktion wurde durch Zugabe von $\mathrm{H}_{2} \mathrm{O}(5 \mathrm{~mL})$ und anschließendes langsames Erwärmen auf RT abgebrochen. Die Phasen wurden getrennt und die wässrige Phase mit $\mathrm{CH}_{2} \mathrm{Cl}_{2}(3 \times 5 \mathrm{~mL})$ extrahiert. Die vereinigten organischen Extrakte wurden über $\mathrm{MgSO}_{4}$ getrocknet und das Lösungsmittel im Vakuum entfernt. Säulenchromatographische Reinigung des Rückstands an Kieselgel (24 g, Petrolether / MTBE 7:1) lieferte die diastereomerenreine Zielverbindung syn-107 (416 mg, 2.02 mmol, 81\%) als farbloses Öl. Zusätzlich konnte das Diastereomer anti-107 (17.0 mg, 82.4 umol, 3\%) ebenfalls als farblöses Öl isoliert werden.

$\mathbf{R}_{f}=0.22$ (Petrolether / MTBE 7:1).

UV $\left(\mathrm{CH}_{3} \mathrm{CN}\right): \lambda_{\max }(\lg \varepsilon)=205 \mathrm{~nm}$ (3.9061), 253 (2.1190), 258 (2.2277).

IR: $\tilde{v}\left(\mathrm{~cm}^{-1}\right)=3565,3443,2976,2871,1640,1497,1454,1374,1067,1027,989,911$, $735,696$.

HPLC (analytisch): Säule: $\quad$ Chiralpak $^{\circledR}$ IA

Eluens: $\quad n$-Hexan / 2-Propanol $=99: 1$

Fluss: $\quad 0.8 \mathrm{~mL} / \mathrm{min}$

$t_{\mathrm{R}}: \quad 14.60 \mathrm{~min}, 86.3 \%$ ee.

${ }^{1} \mathrm{H}-\mathrm{NMR}\left(300 \mathrm{MHz}, \mathrm{CDCl}_{3}\right): \delta(\mathrm{ppm})=1.20\left(\mathrm{~d}, J=6.1 \mathrm{~Hz}, 3 \mathrm{H}, 1-\mathrm{H}_{3}\right), 2.20\left(\mathrm{~m}_{\mathrm{c}}, 1 \mathrm{H}\right.$, 4- $\left.\mathrm{H}_{\mathrm{A}}\right), 2.29-2.40\left(\mathrm{~m}, 1 \mathrm{H}, 4-\mathrm{H}_{\mathrm{B}}\right), 2.54(\mathrm{~d}, J=3.9 \mathrm{~Hz}, 1 \mathrm{H}, \mathrm{OH}), 3.44$ (quint, $J=6.1 \mathrm{~Hz}$, $1 \mathrm{H}, 2-\mathrm{H}), 3.52\left(\mathrm{~m}_{\mathrm{c}}, 1 \mathrm{H}, 3-\mathrm{H}\right), 4.43\left(\mathrm{~d}, J=11.5 \mathrm{~Hz}, 1 \mathrm{H}, \mathrm{OCH}_{\mathrm{A}} \mathrm{Ph}\right), 4.66(\mathrm{~d}, J=11.5 \mathrm{~Hz}$, 
$\left.1 \mathrm{H}, \mathrm{OCH}_{\mathrm{B}} \mathrm{Ph}\right), 5.04-5.14\left(\mathrm{~m}, 2 \mathrm{H}, 6-\mathrm{H}_{2}\right), 5.79-5.94(\mathrm{~m}, 1 \mathrm{H}, 5-\mathrm{H}), 7.23-7.39(\mathrm{~m}, 5 \mathrm{H}$, $\left.\mathrm{Ph}-\mathrm{H}_{5}\right)$.

${ }^{13} \mathrm{C}-\mathrm{NMR}\left(126 \mathrm{MHz}, \mathrm{CDCl}_{3}\right): \delta(\mathrm{ppm})=15.5(\mathrm{C}-1), 37.6(\mathrm{C}-4), 71.0\left(\mathrm{OCH}_{2} \mathrm{Ph}\right), 74.2(\mathrm{C}-3)$, 77.5 (C-2), 117.1 (C-6), $127.6\left(\mathrm{Ph}^{-C_{p}}\right), 127.7$ (2x Ph-C $), 128.3$ (2x Ph- $\left.\mathrm{C}_{m}\right), 134.7$ (C-5), $138.2\left(\mathrm{Ph}-\mathrm{C}_{i}\right)$.

MS (ESI): $m / z(\%)=435.3(100)[2 \mathrm{M}+\mathrm{Na}]^{+}, 413.3(13)[2 \mathrm{M}+\mathrm{H}]^{+}$.

$\mathrm{C}_{13} \mathrm{H}_{18} \mathrm{O}_{\mathbf{2}}$ (206.28) ber.: 229.1199

gef.: $229.1198[\mathrm{M}+\mathrm{Na}]^{+}$(ESI-HRMS).

\section{4 (rac)-Pent-1-en-3-yl-3,5-dinitrobenzoat (118)}

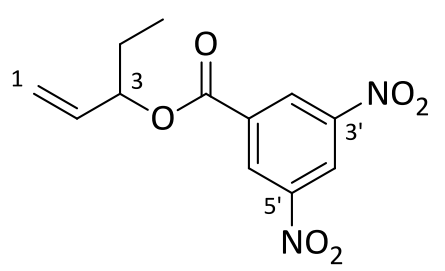

1-Penten-3-ol (116) (1.00 mL, $838 \mathrm{mg}, 9.67 \mathrm{mmol}, 1.00$ Äq.) wurde in absolutem $\mathrm{CH}_{2} \mathrm{Cl}_{2}(40 \mathrm{~mL})$ gelöst und die Lösung bei RT mit NEt $3(2.28 \mathrm{~mL}, 1.66 \mathrm{~g}, 16.4 \mathrm{mmol}$, 1.70 Äq.) sowie DMAP (118 mg, $967 \mu \mathrm{mol}, 10.0$ Mol\%) versetzt. Zu dem Reaktionsgemisch wurde unter Eiskühlung 3,5-Dinitrobenzoylchlorid $(2.90 \mathrm{~g}, 12.6 \mathrm{mmol}$, 1.30 Äq.) gegeben und anschließend wurde für $4 \mathrm{~h}$ bei RT gerührt. Die flüchtigen Bestandteile wurden im Vakuum entfernt und der Rückstand säulenchromatographisch an Kieselgel (200 g, Petrolether / EtOAc 15:1) gereinigt. Die Titelverbindung 118 (1.99 g, 7.10 mmol, 73\%) wurde als gelblicher Feststoff erhalten.

$\mathbf{R}_{f}=0.38$ (Petrolether / EtOAc 13:1).

Smp.: $70.3^{\circ} \mathrm{C}$.

${ }^{1} \mathrm{H}-\mathrm{NMR}\left(600 \mathrm{MHz}, \mathrm{CDCl}_{3}\right): \delta(\mathrm{ppm})=0.99\left(\mathrm{t}, J=7.5 \mathrm{~Hz}, 3 \mathrm{H}, 5-\mathrm{H}_{3}\right), 1.81(\mathrm{ddd}, J=13.9$, 7.5, $\left.6.5 \mathrm{~Hz}, 1 \mathrm{H}, 4-\mathrm{H}_{\mathrm{A}}\right), 1.88\left(\mathrm{~m}_{\mathrm{c}}, 1 \mathrm{H}, 4-\mathrm{H}_{\mathrm{B}}\right), 5.29\left(\mathrm{dt}, J=10.5,1.2 \mathrm{~Hz}, 1 \mathrm{H}, 1-\mathrm{H}_{\mathrm{A}}\right), 5.36$ $\left(\mathrm{dt}, J=17.3,1.2 \mathrm{~Hz}, 1 \mathrm{H}, 1-\mathrm{H}_{\mathrm{B}}\right.$ ), 5.47 (q, $J=7.0 \mathrm{~Hz}, 1 \mathrm{H}, 3-\mathrm{H}$ ), 5.88 (ddd, $J=17.3,10.5$, $7.0 \mathrm{~Hz}, 1 \mathrm{H}, 2-\mathrm{H}$ ), 9.13 (d, J = 2.2 Hz, $\left.2 \mathrm{H}, 2^{\prime}-\mathrm{H}, 6^{\prime}-\mathrm{H}\right), 9.20$ (t, J = $\left.2.2 \mathrm{~Hz}, 1 \mathrm{H}, 4^{\prime}-\mathrm{H}\right)$. 
${ }^{13} \mathrm{C}-\mathrm{NMR}\left(126 \mathrm{MHz}, \mathrm{CDCl}_{3}\right): \delta(\mathrm{ppm})=9.5$ (C-5), 27.2 (C-4), 79.3 (C-3), 118.6 (C-1), 122.3 (C-4'), 129.4 (C-2', C-6'), 134.3 (C-1'), 135.0 (C-2), 148.6 (C-3', C-5'), 161.8 (OC(O)Ar).

MS (EI): $m / z(\%)=281.1(100)[\mathrm{M}+\mathrm{H}]^{+}, 280.1(96)[\mathrm{M}]^{+}$.

$\mathrm{C}_{12} \mathrm{H}_{12} \mathbf{N}_{2} \mathbf{O}_{6}(280.23)$.

\section{5 (S)-Pent-1-en-3-yl-3,5-dinitrobenzoat (118)}<smiles>C=C[C@H](CC)OC(=O)c1cc([N+](=O)[O-])cc([N+](=O)[O-])c1</smiles>

Zu aktiviertem Molsieb $4 \AA$ (500 mg) wurde absolutes $n$-Hexan (30 mL) gegeben und darin nacheinander 1-Penten-3-ol (116) (1.19 mL, $1.00 \mathrm{~g}, 11.6 \mathrm{mmol}, 1.00$ Äq.) sowie Vinylacetat $(2.14 \mathrm{~mL}, 2.00 \mathrm{~g}, 32.2 \mathrm{mmol}, 2.00$ Äq.) gelöst. Das Reaktionsgemisch wurde bei RT mit Amano Lipase AK (500 mg) versetzt und die Suspension für $7 \mathrm{~h}$ bei RT gerührt. Das Enzym wurde abfiltriert und mit absolutem $\mathrm{CH}_{2} \mathrm{Cl}_{2}(30 \mathrm{~mL})$ gewaschen. Das Filtrat wurde bei RT mit NEt $3(1.37 \mathrm{~mL}, 1.00 \mathrm{~g}, 9.87 \mathrm{mmol}, 0.85$ Äq.) sowie DMAP $(70.9 \mathrm{mg}, 5.81 \mu \mathrm{mol}, 5.00 \mathrm{Mol} \%)$ versetzt und auf $0{ }^{\circ} \mathrm{C}$ gekühlt. Nach portionsweiser Zugabe von 3,5-Dinitrobenzoylchlorid (1.74 g, $7.55 \mathrm{mmol}, 0.65$ Äq.) wurde das Reaktionsgemisch für 5 min bei $0^{\circ} \mathrm{C}$ und anschließend $14 \mathrm{~h}$ bei RT gerührt. Die flüchtigen Bestandteile wurden im Vakuum entfernt und der Rückstand säulenchromatographisch an Kieselgel (200 g, Petrolether / MTBE 20:1) gereinigt. Die Titelverbindung 118 (734 mg, $2.62 \mathrm{mmol}, 23 \%$ über 2 Stufen) wurde als gelblicher Feststoff erhalten.

$\mathbf{R}_{f}=0.73$ (Petrolether / EtOAc 4:1).

MS (EI): $m / z(\%)=281.1(100)[\mathrm{M}+\mathrm{H}]^{+}, 280.1(96)[\mathrm{M}]^{+}$.

$\mathrm{C}_{12} \mathrm{H}_{12} \mathrm{~N}_{2} \mathrm{O}_{6}(280.23)$

ber.: 281.0678

gef.: $281.0670[\mathrm{M}+\mathrm{H}]^{+}$(EI-HRMS). 


\subsection{HPLC-Reinigung von (S)-Pent-1-en-3-yl-3,5-dinitrobenzoat (118)}

Verbindung 118 (734 mg, $2.62 \mathrm{mmol})$ wurde in einem Gemisch aus $n$-Hexan $/ \mathrm{CH}_{2} \mathrm{Cl}_{2}$ $(1: 1,2.5 \mathrm{~mL})$ gelöst. Je $100 \mu \mathrm{L}$ dieser Probenlösung (entsprechend $29.4 \mathrm{mg}$ bzw. $105 \mu \mathrm{mol}$ der Verbindung) wurden in das präparative HPLC-System (Säule: Chiralpak ${ }^{\circledR}$ IA, $250 \times 20 \mathrm{~mm}$, Partikelgröße: $5 \mu \mathrm{m}$, mobile Phase: $n$-Hexan / 2-Propanol = 98:2, Fluss: $18 \mathrm{~mL}$ / min, Druck: 6.6 MPa) injiziert. Fraktioniertes Auffangen des Eluats (UVDetektor: $225 \mathrm{~nm}$ ) lieferte die enantiomerenreine Verbindung (S)-118.

Analytische Daten für $(S)-\mathbf{1 1 8}$ :

$\begin{array}{lll}\text { HPLC (präparativ): } & \mathrm{t}_{\mathrm{R}}: & 15.00 \mathrm{~min} \\ & \text { Fraktion: } & 14.7-25.3 \mathrm{~min} \\ \text { HPLC (analytisch): } & \text { Säule: } & \text { Chiralpak }^{\circledR} \mathrm{IA} \\ & \text { Eluens: } & n \text {-Hexan } / 2 \text {-Propanol }=98: 2 \\ & \text { Fluss: } & 1.0 \mathrm{~mL} / \mathrm{min} \\ & \mathrm{t}_{\mathrm{R}}: & 14.9 \mathrm{~min}, 99.9 \% \text { ee. }\end{array}$

$[\alpha]_{\mathrm{D}}^{20}=+28.4^{\circ}\left(c=0.415, \mathrm{CHCl}_{3}\right)$.

Smp.: $80.2^{\circ} \mathrm{C}$.

UV $\left(\mathrm{CH}_{3} \mathrm{CN}\right): \lambda_{\max }(\lg \varepsilon)=209 \mathrm{~nm}$ (4.4272).

IR: $\tilde{v}\left(\mathrm{~cm}^{-1}\right)=3108,2974,2931,2879,1719,1629,1537,1458,1278,1171,1072,923$, $902,729,716$.

${ }^{1} \mathbf{H}-\mathbf{N M R}\left(300 \mathrm{MHz}, \mathrm{CDCl}_{3}\right): \delta(\mathrm{ppm})=0.99\left(\mathrm{t}, J=7.4 \mathrm{~Hz}, 3 \mathrm{H}, 5-\mathrm{H}_{3}\right), 1.72-1.97(\mathrm{~m}, 2 \mathrm{H}$, 4- $\left.\mathrm{H}_{2}\right), 5.29\left(\mathrm{dt}, J=10.5,1.1 \mathrm{~Hz}, 1 \mathrm{H}, 1-\mathrm{H}_{\mathrm{A}}\right), 5.36\left(\mathrm{dt}, J=17.3,1.1 \mathrm{~Hz}, 1 \mathrm{H}, 1-\mathrm{H}_{\mathrm{B}}\right), 5.48$ (q, $J=6.9 \mathrm{~Hz}, 1 \mathrm{H}, 3-\mathrm{H}), 5.89(\mathrm{ddd}, J=17.3,10.5,6.9 \mathrm{~Hz}, 1 \mathrm{H}, 2-\mathrm{H}), 9.14(\mathrm{~d}, J=2.2 \mathrm{~Hz}, 2 \mathrm{H}$, 2'-H, 6'-H), 9.20 (t, J = $\left.2.2 \mathrm{~Hz}, 1 \mathrm{H}, 4^{\prime}-\mathrm{H}\right)$.

${ }^{13} \mathrm{C}-\mathrm{NMR}\left(126 \mathrm{MHz}, \mathrm{CDCl}_{3}\right): \delta(\mathrm{ppm})=9.5$ (C-5), 27.2 (C-4), 79.3 (C-3), 118.7 (C-1), 122.3 (C-4'), 129.4 (C-2', C-6'), 134.3 (C-1'), 135.0 (C-2), 148.7 (C-3', C-5'), 161.8 (OC(O)Ar). 
6.7 (2S,3S,7S)-2-(Benzyloxy)-3-( $N, N$-didemethyl- $N$-benzyloxycarbonyl- $\alpha$-Dforosaminyl)-non-5-en-7-yl-3,5-dinitrobenzoat ( $\alpha-119)$

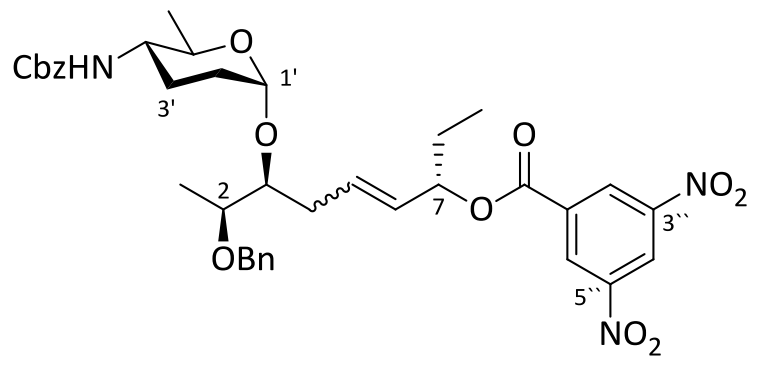

Das Glykosid $\alpha-115(100 \mathrm{mg}, 220 \mu \mathrm{mol}, 1.00$ Äq.) sowie das Dinitrobenzoat 118 (92.7 mg, $331 \mu \mathrm{mol}, 1.50$ Äq.) wurden in absolutem $\mathrm{CH}_{2} \mathrm{Cl}_{2}(16 \mathrm{~mL})$ gelöst und die Lösung auf $0{ }^{\circ} \mathrm{C}$ gekühlt. Zu dem Reaktionsgemisch wurde tropfenweise GrubbsKatalysator 2. Generation $(28.1 \mathrm{mg}, 33.1 \mu \mathrm{mol}, 15.0 \mathrm{Mol} \%)$, gelöst in absolutem $\mathrm{CH}_{2} \mathrm{Cl}_{2}(6 \mathrm{~mL})$, gegeben und anschließend für $18 \mathrm{~h}$ unter Rückfluss gerührt. Das Lösungsmittel wurde im Vakuum entfernt und der Rückstand säulenchromatographisch an Kieselgel (25 g, Petrolether / EtOAc 5:1) gereinigt. Die Zielverbindung wurde als Gemisch der Doppelbindungsisomere $(E)-\alpha-119$ / (Z)- $\alpha-119$ ( 7:1, 114 mg, $162 \mu \mathrm{mol}, 74 \%)$ in Form eines gelb-braunen Schaums erhalten.

$\mathbf{R}_{f}=0.42$ (Petrolether / EtOAc 3:1).

UV $\left(\mathrm{CH}_{3} \mathrm{CN}\right): \lambda_{\max }(\lg \varepsilon)=206 \mathrm{~nm}$ (4.6023).

IR: $\tilde{v}\left(\mathrm{~cm}^{-1}\right)=3403,3326,2971,2934,2877,1722,1629,1543,1455,1343,1271$, 1072, 1024, 985, 730, 720, 697.

${ }^{1} \mathrm{H}-\mathrm{NMR}\left(600 \mathrm{MHz}, \mathrm{CDCl}_{3}, E\right.$-Isomer beschrieben): $\delta(\mathrm{ppm})=0.95(\mathrm{t}, J=7.4 \mathrm{~Hz}, 3 \mathrm{H}$, 9- $\left.\mathrm{H}_{3}\right), 1.02\left(\mathrm{~d}, J=6.3 \mathrm{~Hz}, 3 \mathrm{H}, 6^{\prime}-\mathrm{H}_{3}\right), 1.16\left(\mathrm{~d}, J=6.4 \mathrm{~Hz}, 3 \mathrm{H}, 1-\mathrm{H}_{3}\right), 1.49-1.56(\mathrm{~m}, 1 \mathrm{H}$, $\left.3^{\prime}-H_{A}\right), 1.59-1.67\left(m, 1 \mathrm{H}, 2^{\prime}-H_{A}\right), 1.67-1.73\left(m, 2 \mathrm{H}, 2^{\prime}-\mathrm{H}_{B}, 3^{\prime} \mathrm{H}_{B}\right), 1.75\left(m_{c}, 1 \mathrm{H}, 8-\mathrm{H}_{A}\right)$, $1.85\left(m_{c}, 1 \mathrm{H}, 8-H_{B}\right), 2.20\left(d t, J=14.6,7.3 \mathrm{~Hz}, 1 \mathrm{H}, 4-\mathrm{H}_{A}\right), 2.42-2.48\left(\mathrm{~m}, 1 \mathrm{H}, 4-\mathrm{H}_{\mathrm{B}}\right), 3.29$ (dt, $\left.J=13.4,3.9 \mathrm{~Hz}, 1 \mathrm{H}, 4^{\prime}-\mathrm{H}\right), 3.53\left(\mathrm{dt}, J=15.8,6.3 \mathrm{~Hz}, 1 \mathrm{H}, 5^{\prime}-\mathrm{H}\right), 3.59\left(\mathrm{~m}_{\mathrm{c}}, 1 \mathrm{H}, 2-\mathrm{H}\right)$, $3.70(\mathrm{dd}, J=11.2,5.1 \mathrm{~Hz}, 1 \mathrm{H}, 3-\mathrm{H}), 4.21(\mathrm{~d}, J=9.5 \mathrm{~Hz}, 1 \mathrm{H}, \mathrm{NH}), 4.46(\mathrm{~d}, J=11.9 \mathrm{~Hz}$, $\left.1 \mathrm{H}, \mathrm{OCH}_{\mathrm{A}} \mathrm{Ph}\right), 4.61\left(\mathrm{~d}, J=11.9 \mathrm{~Hz}, 1 \mathrm{H}, \mathrm{OCH}_{\mathrm{B}} \mathrm{Ph}\right), 4.80\left(\mathrm{~m}_{\mathrm{c}}, 1 \mathrm{H}, 1^{\prime}-\mathrm{H}\right), 5.04(\mathrm{~s}, 2 \mathrm{H}$, $\mathrm{NC}(\mathrm{O}) \mathrm{OCH}_{2} \mathrm{Ph}$ ), 5.40 (dd, $\left.J=14.2,7.0 \mathrm{~Hz}, 1 \mathrm{H}, 7-\mathrm{H}\right), 5.54(\mathrm{dd}, J=15.4,7.8 \mathrm{~Hz}, 1 \mathrm{H}$, 
6-H), $5.82\left(\mathrm{~m}_{\mathrm{c}}, 1 \mathrm{H}, 5-\mathrm{H}\right), 7.16-7.36\left(\mathrm{~m}, 10 \mathrm{H}, 2 \times \mathrm{Ph}-\mathrm{H}_{5}\right), 9.09$ (d, J = 2.1 Hz, $2 \mathrm{H}, 2$ "-H, 6"-H), 9.18 (t, J = $\left.2.1 \mathrm{~Hz}, 1 \mathrm{H}, 4^{\prime \prime}-\mathrm{H}\right)$.

${ }^{13} \mathrm{C}-\mathrm{NMR}\left(126 \mathrm{MHz}, \mathrm{CDCl}_{3}\right.$, E-Isomer beschrieben): $\delta(\mathrm{ppm})=9.6$ (C-9), 14.9 (C-1), 18.0 (C-6'), 25.6 (C-3'), 27.4 (C-8), 29.6 (C-2'), 31.9 (C-4), 52.2 (C-4'), 66.6 (NC(O)OC $\left.\mathrm{H}_{2} \mathrm{Ph}\right)$, 68.7 (C-5'), $71.2\left(\mathrm{OCH}_{2} \mathrm{Ph}\right), 75.7$ (C-2), 77.4 (C-3), 79.4 (C-7), 95.1 (C-1'), 122.2 (C-4"), 127.5, 127.6, 128.0, 128.1, 128.3, 128.4 (4 x Ph-C 4 x Ph- $C_{m}, 2$ x Ph- $C_{p}$ ), 129.2 (C-2", C-6"), 129.5 (C-6), 132.5 (C-5), 134.3 (C-1"), 136.4 (NC(O)OCH$\left.{ }_{2} \mathrm{Ph}_{-} \underline{\mathrm{C}}_{i}\right), 138.7$ $\left(\mathrm{OCH}_{2} \mathrm{Ph}-\underline{\mathrm{C}}_{i}\right), 148.6$ (C-3", C-5"), 155.7 ( $\left.\mathrm{N} \underline{\mathrm{C}}(\mathrm{O}) \mathrm{OCH}_{2} \mathrm{Ph}\right), 161.7$ (OC(O)Ar).

MS (ESI): $m / z(\%)=728.3(100)[\mathrm{M}+\mathrm{Na}]^{+}, 534.3(17)\left[\mathrm{M}-\mathrm{C}_{7} \mathrm{H}_{2} \mathrm{~N}_{2} \mathrm{O}_{5}+\mathrm{Na}\right]^{+}$.

$\mathrm{C}_{37} \mathrm{H}_{43} \mathbf{N}_{3} \mathbf{O}_{11}(705.75) \quad$ ber.: 728.2790

gef.: $728.2784[\mathrm{M}+\mathrm{Na}]^{+}$(ESI-HRMS).

\section{8 (2S,3S,7S)-2-(Benzyloxy)-3-( $N, N$-didemethyl- $N$-benzyloxycarbonyl- $\beta$-D- forosaminyl)-non-5-en-7-yl-3,5-dinitrobenzoat ( $\beta$-119)}

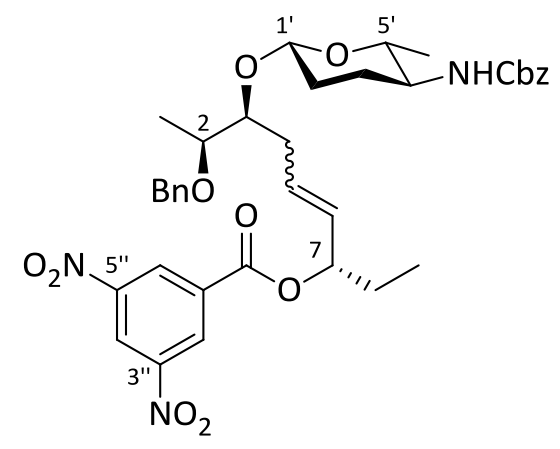

Das Glykosid $\beta-115$ (97.0 mg, $214 \mu \mathrm{mol}, 1.00$ Äq.) sowie das Dinitrobenzoat 118 (95.9 mg, $342 \mu \mathrm{mol}, 1.60$ Äq.) wurden in absolutem $\mathrm{CH}_{2} \mathrm{Cl}_{2}(20 \mathrm{~mL})$ gelöst und die Lösung auf $0{ }^{\circ} \mathrm{C}$ gekühlt. Zu dem Reaktionsgemisch wurde tropfenweise GrubbsKatalysator 2 . Generation $(27.2 \mathrm{mg}, 32.1 \mu \mathrm{mol}, 15.0 \mathrm{Mol} \%)$, gelöst in absolutem $\mathrm{CH}_{2} \mathrm{Cl}_{2}(5 \mathrm{~mL})$, gegeben und anschließend für $26 \mathrm{~h}$ unter Rückfluss gerührt. Das Lösungsmittel wurde im Vakuum entfernt und der Rückstand säulenchromatographisch an Kieselgel (23 g, Petrolether / EtOAc 5:1) gereinigt. Die Zielverbindung wurde als Gemisch der Doppelbindungsisomere $(E)-\beta-119 /(Z)-\beta-119$ ( $\approx: 1,105 \mathrm{mg}$, $149 \mu \mathrm{mol}, 70 \%)$ in Form eines gelb-braunen Schaums erhalten. 
$\mathbf{R}_{f}=0.39$ (Petrolether / EtOAc 3:1).

UV $\left(\mathrm{CH}_{3} \mathrm{CN}\right): \lambda_{\max }(\lg \varepsilon)=206 \mathrm{~nm}$ (4.6525).

IR: $\tilde{v}\left(\mathrm{~cm}^{-1}\right)=3329,2972,2934,2865,1721,1629,1543,1455,1343,1273,1167$, 1066, 730, 720, 697.

${ }^{1} \mathrm{H}-\mathrm{NMR}\left(600 \mathrm{MHz}, \mathrm{C}_{6} \mathrm{D}_{6}, \quad\right.$ E-Isomer beschrieben): $\delta(\mathrm{ppm})=0.85(\mathrm{t}, J=7.4 \mathrm{~Hz}, 3 \mathrm{H}$, 9- $\left.\mathrm{H}_{3}\right), 1.06\left(\mathrm{~d}, J=6.3 \mathrm{~Hz}, 3 \mathrm{H}, 1-\mathrm{H}_{3}\right), 1.20\left(\mathrm{~d}, J=6.1 \mathrm{~Hz}, 3 \mathrm{H}, 6^{\prime}-\mathrm{H}_{3}\right), 1.45-1.65(\mathrm{~m}, 5 \mathrm{H}$, 8- $\left.\mathrm{H}_{\mathrm{A}}, 2^{\prime}-\mathrm{H}_{2}, 3^{\prime}-\mathrm{H}_{2}\right), 1.69\left(\mathrm{~m}_{\mathrm{c}}, 1 \mathrm{H}, 8-\mathrm{H}_{\mathrm{B}}\right), 2.27\left(\mathrm{~m}_{\mathrm{c}}, 1 \mathrm{H}, 4-\mathrm{H}_{\mathrm{A}}\right), 2.45-2.54\left(\mathrm{~m}, 1 \mathrm{H}, 4-\mathrm{H}_{B}\right)$, $2.79\left(\mathrm{dq}, J=12.3,6.1 \mathrm{~Hz}, 1 \mathrm{H}, 5^{\prime}-\mathrm{H}\right), 3.16\left(\mathrm{~m}_{\mathrm{c}}, 1 \mathrm{H}, 4^{\prime}-\mathrm{H}\right), 3.50$ (quint, $J=6.2 \mathrm{~Hz}, 1 \mathrm{H}$, 2-H), $3.84\left(m_{c}, 1 \mathrm{H}, 3-\mathrm{H}\right), 3.92(\mathrm{~d}, J=9.3 \mathrm{~Hz}, 1 \mathrm{H}, \mathrm{NH}), 4.33(\mathrm{~d}, J=11.9 \mathrm{~Hz}, 1 \mathrm{H}$, $\left.\mathrm{OCH}_{\mathrm{A}} \mathrm{Ph}\right), 4.44\left(\mathrm{~d}, J=8.7 \mathrm{~Hz}, 1 \mathrm{H}, 1^{\prime}-\mathrm{H}\right), 4.47\left(\mathrm{~d}, J=11.9 \mathrm{~Hz}, 1 \mathrm{H}, \mathrm{OCH}_{\mathrm{B}} \mathrm{Ph}\right), 5.04(\mathrm{~s}, 2 \mathrm{H}$, $\left.\mathrm{NC}(\mathrm{O}) \mathrm{OCH}_{2} \mathrm{Ph}\right), 5.48-5.56(\mathrm{~m}, 2 \mathrm{H}, 6-\mathrm{H}, 7-\mathrm{H}), 6.17\left(\mathrm{~m}_{\mathrm{c}}, 1 \mathrm{H}, 5-\mathrm{H}\right), 7.03-7.35(\mathrm{~m}, 10 \mathrm{H}$, $\left.2 \times \mathrm{Ph}^{-\mathrm{H}_{5}}\right), 8.59\left(\mathrm{~s}_{\mathrm{br}}, 1 \mathrm{H}, 4\right.$ "-H), 8.79 (d, J = $1.2 \mathrm{~Hz}, 2 \mathrm{H}, 2$ "-H, 6"-H).

${ }^{13} \mathrm{C}-\mathrm{NMR}\left(126 \mathrm{MHz}, \mathrm{C}_{6} \mathrm{D}_{6}\right.$, E-Isomer beschrieben): $\delta(\mathrm{ppm})=9.7$ (C-9), 15.2 (C-1), 18.7 (C-6'), 27.6 (C-8), 29.4 (C-3'), 31.5 (C-2'), 34.3 (C-4), 52.5 (C-4'), 66.7 (NC(O)OC_H $\left.{ }_{2} \mathrm{Ph}\right)$, $71.4\left(\mathrm{OCH}_{2} \mathrm{Ph}\right), 75.0$ (C-5'), 76.9 (C-2), 79.1 (C-3), 79.2 (C-7), 101.6 (C-1'), 122.0 (C-4"), 127.6, 127.7, 128.3, 128.5, 128.5, 128.7 (4 x Ph-C , $_{0}$ x Ph- $\mathrm{C}_{m}, 2$ x Ph- $\mathrm{C}_{p}$ ), 128.6 (C-2", C-6"), 128.8 (C-6), 133.2 (C-5), 133.9 (C-1"), 137.3 (NC(O)OCH$\left.{ }_{2} \mathrm{Ph}_{-} \underline{C}_{i}\right), 139.4$

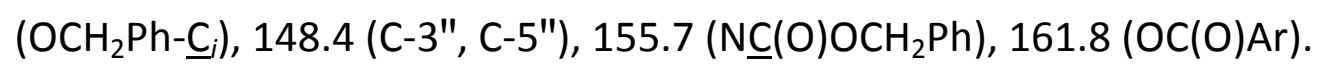

MS (ESI): $m / z(\%)=728.3(100)[\mathrm{M}+\mathrm{Na}]^{+}, 534.3(16)\left[\mathrm{M}-\mathrm{C}_{7} \mathrm{H}_{2} \mathrm{~N}_{2} \mathrm{O}_{5}+\mathrm{Na}\right]^{+}$.

$\mathrm{C}_{37} \mathrm{H}_{43} \mathbf{N}_{3} \mathbf{O}_{11}$ (705.75) ber.: 728.2790

gef.: $728.2784[\mathrm{M}+\mathrm{Na}]^{+}$(ESI-HRMS).

$6.9 \quad(2 S, 3 S, 7 S)-2-(B e n z y l o x y)-3-(N, N$-didemethyl- $N$-benzyloxycarbonyl- $\alpha-D-$ forosaminyl)-non-5-en-7-ol ( $\alpha$-120)

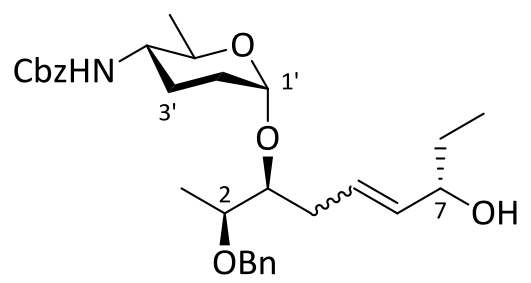

Das Dinitrobenzoat $\alpha-119$ (114 mg, $162 \mu \mathrm{mol}, 1.00$ Äq.) wurde in absolutem $\mathrm{MeOH} / \mathrm{THF}(5: 1,12 \mathrm{~mL})$ gelöst und die Lösung auf $0{ }^{\circ} \mathrm{C}$ gekühlt. Nach der Zugabe von 
$\mathrm{K}_{2} \mathrm{CO}_{3}$ (112 mg, $808 \mu \mathrm{mol}, 4.99$ Äq.) wurde das Reaktionsgemisch für $45 \mathrm{~min}$ bei $0{ }^{\circ} \mathrm{C}$ gerührt. Anschließend wurde mit MTBE $(20 \mathrm{~mL})$ verdünnt und mit ges. wässriger $\mathrm{NH}_{4} \mathrm{Cl}$-Lsg. $(15 \mathrm{~mL})$ versetzt. Die organische Phase wurde abgetrennt, über $\mathrm{MgSO}_{4}$ getrocknet und das Lösungsmittel im Vakuum entfernt. Säulenchromatographische Reinigung an Kieselgel (6 g, Petrolether / EtOAc 2:1) lieferte die Titelverbindung als Gemisch der Doppelbindungsisomere $(E)-\alpha-120$ / (Z)- $\alpha-120$ ( 7:1, 76.8 mg, $150 \mu \mathrm{mol}$, 93\%) in Form eines gelblichen Öls.

$\mathbf{R}_{f}=0.24$ (Petrolether / EtOAc 2:1).

UV $\left(\mathrm{CH}_{3} \mathrm{CN}\right): \lambda_{\max }(\lg \varepsilon)=252 \mathrm{~nm}$ (2.8742), 258 (3.8866).

IR: $\tilde{v}\left(\mathrm{~cm}^{-1}\right)=3401,3319,2958,2930,2873,1698,1535,1454,1302,1233,1119$, 1094, 1068, 1023, 984, 734, 696.

${ }^{1} \mathrm{H}-\mathrm{NMR}\left(600 \mathrm{MHz}, \mathrm{CDCl}_{3}\right.$, E-Isomer beschrieben): $\delta(\mathrm{ppm})=0.87(\mathrm{t}, J=7.4 \mathrm{~Hz}, 3 \mathrm{H}$, 9- $\left.\mathrm{H}_{3}\right), 1.03\left(\mathrm{~d}, J=6.2 \mathrm{~Hz}, 3 \mathrm{H}, 6^{\prime}-\mathrm{H}_{3}\right), 1.17\left(\mathrm{~d}, J=6.3 \mathrm{~Hz}, 3 \mathrm{H}, 1-\mathrm{H}_{3}\right), 1.43-1.60(\mathrm{~m}, 3 \mathrm{H}$, 8- $\left.\mathrm{H}_{2}, 3^{\prime}-\mathrm{H}_{\mathrm{A}}\right), 1.71\left(\mathrm{~m}_{\mathrm{c}}, 1 \mathrm{H}, 3^{\prime}-\mathrm{H}_{\mathrm{B}}\right), 1.76\left(\mathrm{~m}_{\mathrm{c}}, 2 \mathrm{H}, 2^{\prime}-\mathrm{H}_{2}\right), 2.15\left(\mathrm{~m}_{\mathrm{c}}, 1 \mathrm{H}, 4-\mathrm{H}_{\mathrm{A}}\right), 2.41\left(\mathrm{~m}_{\mathrm{c}}\right.$, $\left.1 \mathrm{H}, 4-\mathrm{H}_{B}\right), 3.31\left(\mathrm{~m}_{\mathrm{c}}, 1 \mathrm{H}, 4^{\prime}-\mathrm{H}\right), 3.53-3.61\left(\mathrm{~m}, 2 \mathrm{H}, 2-\mathrm{H}, 5^{\prime}-\mathrm{H}\right), 3.67\left(\mathrm{~m}_{\mathrm{c}}, 1 \mathrm{H}, 3-\mathrm{H}\right), 3.93$ $\left(m_{c}, 1 \mathrm{H}, 7-\mathrm{H}\right), 4.13(\mathrm{~d}, J=10.0 \mathrm{~Hz}, 1 \mathrm{H}, \mathrm{NH}), 4.46$ (d, J=11.8 Hz, $\left.1 \mathrm{H}, \mathrm{OCH}_{\mathrm{A}} \mathrm{Ph}\right), 4.63(\mathrm{~d}$, $\left.J=11.8 \mathrm{~Hz}, 1 \mathrm{H}, \mathrm{OCH}_{\mathrm{B}} \mathrm{Ph}\right), 4.84\left(\mathrm{~s}_{\mathrm{br}}, 1 \mathrm{H}, 1^{\prime}-\mathrm{H}\right), 5.05\left(\mathrm{~s}, 2 \mathrm{H}, \mathrm{NC}(\mathrm{O}) \mathrm{OCH}_{2} \mathrm{Ph}\right), 5.47$ (dd, $J=15.5,6.7 \mathrm{~Hz}, 1 \mathrm{H}, 6-\mathrm{H}), 5.55-5.61(\mathrm{~m}, 1 \mathrm{H}, 5-\mathrm{H}), 7.16-7.38\left(\mathrm{~m}, 10 \mathrm{H}, 2 \times \mathrm{Ph}^{-\mathrm{H}_{5}}\right)$.

${ }^{13}$ C-NMR (126 MHz, CDCl, E-Isomer beschrieben): $\delta(p p m)=9.7$ (C-9), $15.3(\mathrm{C}-1), 18.1$ (C-6'), 25.7 (C-3'), 29.6 (C-2'), 30.1 (C-8), 31.7 (C-4), 52.3 (C-4'), 66.7 (NC(O)OC $\left.\mathrm{H}_{2} \mathrm{Ph}\right)$, 68.6 (C-5'), $71.3\left(\mathrm{OCH}_{2} \mathrm{Ph}\right), 74.2$ (C-7), 75.8 (C-2), 77.6 (C-3), 94.6 (C-1'), 127.4 (C-5), 127.6, 127.7, 128.0, 128.1, 128.3, 128.5 (4 x Ph- $\mathrm{C}_{o}, 4 \times \mathrm{Ph}-\mathrm{C}_{m}, 2 \times \mathrm{Ph}-\mathrm{C}_{p}$ ), 135.5 (C-6), 136.5, $138.8\left(2 \times \mathrm{Ph}-\mathrm{C}_{i}\right), 155.8\left(\mathrm{~N} \underline{\mathrm{C}}(\mathrm{O}) \mathrm{OCH}_{2} \mathrm{Ph}\right)$.

MS (ESI): $m / z(\%)=534.3(100)[\mathrm{M}+\mathrm{Na}]^{+}$.

$\mathrm{C}_{30} \mathrm{H}_{41} \mathrm{NO}_{6}$ (511.65)

ber.: 534.2826

gef.: $\quad 534.2826[\mathrm{M}+\mathrm{Na}]^{+}$(ESI-HRMS). 
$6.10(2 S, 3 S, 7 S)$-2-(Benzyloxy)-3-( $N, N$-didemethyl- $N$-benzyloxycarbonyl- $\beta$-Dforosaminyl)-non-5-en-7-ol ( $\beta$-120)

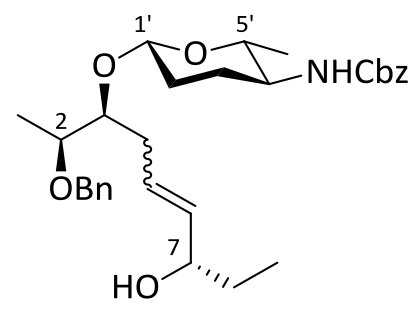

Das Dinitrobenzoat $\beta-119$ (105 mg, $149 \mu \mathrm{mol}, 1.00$ Äq.) wurde in absolutem $\mathrm{MeOH} / \mathrm{THF}(5: 1,10 \mathrm{~mL})$ gelöst und die Lösung auf $0{ }^{\circ} \mathrm{C}$ gekühlt. Nach der Zugabe von $\mathrm{K}_{2} \mathrm{CO}_{3}\left(103 \mathrm{mg}, 744 \mu \mathrm{mol}, 4.99\right.$ Äq.) wurde das Reaktionsgemisch für $45 \mathrm{~min}$ bei $0^{\circ} \mathrm{C}$ gerührt. Anschließend wurde mit MTBE $(20 \mathrm{~mL})$ verdünnt und mit ges. wässriger $\mathrm{NH}_{4} \mathrm{Cl}$-Lsg. (15 mL) versetzt. Die organische Phase wurde abgetrennt, über $\mathrm{MgSO}_{4}$ getrocknet und das Lösungsmittel im Vakuum entfernt. Nach säulenchromatographischer Reinigung des Rückstands an Kieselgel (6.4 g, Petrolether / EtOAc 2:1) wurde die Titelverbindung als Gemisch der Doppelbindungsisomere

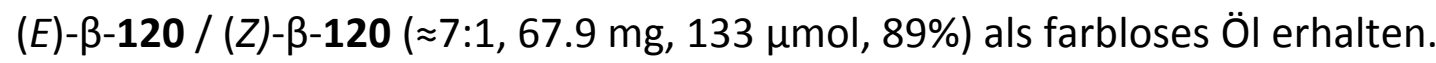

$\mathbf{R}_{f}=0.23$ (Petrolether / EtOAc 2:1).

UV $\left(\mathrm{CH}_{3} \mathrm{CN}\right): \lambda_{\max }(\lg \varepsilon)=252 \mathrm{~nm}$ (2.9386), 257 (2.9396).

IR: $\tilde{v}\left(\mathrm{~cm}^{-1}\right)=3312,2958,2931,2871,1696,1537,1454,1301,1241,1132,1062$, 1025, 984, 736, 696.

${ }^{1} \mathrm{H}-\mathrm{NMR}\left(600 \mathrm{MHz}, \mathrm{CDCl}_{3}, E\right.$-Isomer beschrieben): $\delta(\mathrm{ppm})=0.87$ (t, $J=7.4 \mathrm{~Hz}, 3 \mathrm{H}$, 9- $\left.\mathrm{H}_{3}\right), 1.13\left(\mathrm{~d}, J=6.3 \mathrm{~Hz}, 3 \mathrm{H}, 1-\mathrm{H}_{3}\right), 1.19-1.28\left(\mathrm{~m}, 1 \mathrm{H}, 3^{\prime}-\mathrm{H}_{\mathrm{A}}\right), 1.22(\mathrm{~d}, J=5.9 \mathrm{~Hz}, 3 \mathrm{H}$, $\left.6^{\prime}-\mathrm{H}_{3}\right), 1.48\left(\mathrm{dt}, J=13.6,7.5 \mathrm{~Hz}, 1 \mathrm{H}, 8-\mathrm{H}_{A}\right), 1.51-1.62\left(\mathrm{~m}, 2 \mathrm{H}, 8-\mathrm{H}_{B}, 2^{\prime}-\mathrm{H}_{\mathrm{A}}\right), 1.80\left(\mathrm{~m}_{\mathrm{c}}\right.$, $\left.1 \mathrm{H}, 2^{\prime}-\mathrm{H}_{B}\right), 2.01\left(m_{c}, 1 \mathrm{H}, 3^{\prime}-H_{B}\right), 2.19\left(m_{c}, 1 \mathrm{H}, 4-H_{A}\right), 2.36\left(m_{c}, 1 \mathrm{H}, 4-H_{B}\right), 3.20\left(m_{c}\right.$, $1 \mathrm{H}, 5^{\prime}-\mathrm{H}$ ), $3.31\left(\mathrm{~m}_{\mathrm{c}}, 1 \mathrm{H}, 4^{\prime}-\mathrm{H}\right.$ ), 3.51 (quint, $\left.J=6.2 \mathrm{~Hz}, 1 \mathrm{H}, 2-\mathrm{H}\right), 3.58-3.63(\mathrm{~m}, 1 \mathrm{H}$, 3-H), $3.93(\mathrm{q}, J=6.6 \mathrm{~Hz}, 1 \mathrm{H}, 7-\mathrm{H}), 4.42(\mathrm{~d}, J=8.6 \mathrm{~Hz}, 1 \mathrm{H}, \mathrm{NH}), 4.46(\mathrm{~d}, J=11.7 \mathrm{~Hz}$, $1 \mathrm{H}, \mathrm{OCH}_{\mathrm{A}} \mathrm{Ph}$ ), $4.51\left(\mathrm{~d}, J=9.1 \mathrm{~Hz}, 1 \mathrm{H}, 1^{\prime}-\mathrm{H}\right), 4.60\left(\mathrm{~d}, J=11.8 \mathrm{~Hz}, 1 \mathrm{H}, \mathrm{OCH}_{B} \mathrm{Ph}\right), 5.06(\mathrm{~s}$, $2 \mathrm{H}, \mathrm{NC}(\mathrm{O}) \mathrm{OCH}_{2} \mathrm{Ph}$ ), $5.46(\mathrm{dd}, J=15.5,7.1 \mathrm{~Hz}, 1 \mathrm{H}, 6-\mathrm{H}), 5.66-5.74(\mathrm{~m}, 1 \mathrm{H}, 5-\mathrm{H}), 7.24-$ $7.36\left(\mathrm{~m}, 10 \mathrm{H}, 2 \times \mathrm{Ph}-\mathrm{H}_{5}\right)$. 
${ }^{13}$ C-NMR (126 MHz, CDCl 3 , E-Isomer beschrieben): $\delta(\mathrm{ppm})=9.8$ (C-9), 15.5 (C-1), 18.5 (C-6'), 29.7 (C-3'), 29.8 (C-8), 31.0 (C-2'), 34.1 (C-4), 52.3 (C-4'), 66.8 (NC(O)OC $\left.\mathrm{CH}_{2} \mathrm{Ph}\right)$, $71.4\left(\mathrm{OCH}_{2} \mathrm{Ph}\right), 74.3$ (C-7), 75.1 (C-5'), 76.6 (C-2), 80.8 (C-3), 102.3 (C-1'), 127.5, 127.6, 128.1, 128.2, 128.3, 128.5 (4 x Ph-C $0,4 \times \mathrm{Ph}-\mathrm{C}_{m}, 2 \times \mathrm{Ph}-\mathrm{C}_{p}$ ), 128.7 (C-5), 134.8 (C-6), 136.4, $138.7\left(2 \times \mathrm{Ph}-\mathrm{C}_{i}\right), 155.7\left(\mathrm{~N} \underline{\mathrm{C}}(\mathrm{O}) \mathrm{OCH}_{2} \mathrm{Ph}\right)$.

MS (ESI): $m / z(\%)=1045.6(100)[2 \mathrm{M}+\mathrm{Na}]^{+}, 534.3(77)[\mathrm{M}+\mathrm{Na}]^{+}$.

$\mathrm{C}_{30} \mathrm{H}_{41} \mathrm{NO}_{6}(511.65) \quad$ ber.: 534.2826

gef.: $\quad 534.2827[\mathrm{M}+\mathrm{Na}]^{+}$(ESI-HRMS).

\subsection{1 (2S,3S,7S)-2-(Benzyloxy)-7-((tert-butyldimethylsilyl)oxy)-3-(N,N-dide- methyl- $N$-benzyloxycarbonyl- $\alpha$-D-forosaminyl)-non-5-en ( $\alpha-121)$}

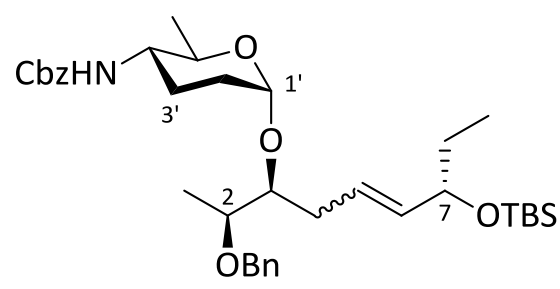

Der Alkohol $\alpha-120$ (107 mg, $209 \mu \mathrm{mol}, 1.00$ Äq.) wurde in absolutem $\mathrm{CH}_{2} \mathrm{Cl}_{2}(15 \mathrm{~mL})$ gelöst und bei RT mit 2,6-Lutidin (48.7 $\mu \mathrm{L}, 44.8 \mathrm{mg}, 418 \mathrm{mmol}, 2.00$ Äq.) versetzt. Die Reaktionslösung wurde auf $-10^{\circ} \mathrm{C}$ gekühlt, TBSOTf $(72.0 \mu \mathrm{L}, 82.9 \mathrm{mg}, 314 \mu \mathrm{mol}$, 1.50 Äq.) zugetropft und für $30 \mathrm{~min}$ bei $-10{ }^{\circ} \mathrm{C}$ gerührt. Durch Zugabe von $\mathrm{H}_{2} \mathrm{O}$ $(30 \mathrm{~mL})$ und langsames Erwärmen auf RT wurde die Reaktion abgebrochen. Die Phasen wurden getrennt und die wässrige Phase mit $\mathrm{CH}_{2} \mathrm{Cl}_{2}(3 \times 30 \mathrm{~mL})$ extrahiert. Die vereinigten organischen Phasen wurden über $\mathrm{MgSO}_{4}$ getrocknet und das Lösungsmittel im Vakuum entfernt. Der Rückstand wurde säulenchromatographisch an Kieselgel (23 g, Petrolether / MTBE 5:1) gereinigt und die Zielverbindung als Gemisch der Doppelbindungsisomere $(E)-\alpha-121 /(Z)-\alpha-121$ ( $7: 1,123$ mg, $197 \mu \mathrm{mol}$, 94\%) als farbloses Öl erhalten.

$\mathbf{R}_{f}=0.39$ (Petrolether / MTBE 3:1).

$\mathrm{UV}\left(\mathrm{CH}_{3} \mathrm{CN}\right): \lambda_{\max }(\lg \varepsilon)=252 \mathrm{~nm}$ (2.9401), 257 (2.9464). 
IR: $\tilde{v}\left(\mathrm{~cm}^{-1}\right)=3321,2954,2929,2856,1699,1515,1455,1303,1248,1120,1078$, $1024,986,835,774,735,696$.

${ }^{1} \mathrm{H}-\mathrm{NMR}\left(600 \mathrm{MHz}, \mathrm{CDCl}_{3}, \quad\right.$-Isomer beschrieben): $\delta(\mathrm{ppm})=-0.01,0.02 \quad(\mathrm{~s}, \quad 6 \mathrm{H}$, $\left.\mathrm{Si}\left(\mathrm{CH}_{3}\right)_{2}\right), 0.82\left(\mathrm{t}, J=7.4 \mathrm{~Hz}, 3 \mathrm{H}, 9-\mathrm{H}_{3}\right), 0.87\left(\mathrm{~s}, 9 \mathrm{H}, \mathrm{SiC}\left(\mathrm{CH}_{3}\right)_{3}\right), 1.02(\mathrm{~d}, J=6.2 \mathrm{~Hz}, 3 \mathrm{H}$, 6'- $\left.\mathrm{H}_{3}\right), 1.16\left(\mathrm{~d}, \mathrm{~J}=6.3 \mathrm{~Hz}, 3 \mathrm{H}, 1-\mathrm{H}_{3}\right), 1.43\left(\mathrm{~m}_{\mathrm{c}}, 2 \mathrm{H}, 8-\mathrm{H}_{2}\right), 1.53(\mathrm{dt}, J=12.1,7.4 \mathrm{~Hz}, 1 \mathrm{H}$, $\left.3^{\prime}-\mathrm{H}_{\mathrm{A}}\right), 1.67-1.72\left(\mathrm{~m}, 1 \mathrm{H}, 3^{\prime}-\mathrm{H}_{\mathrm{B}}\right), 1.72-1.78\left(\mathrm{~m}, 2 \mathrm{H}, 2^{\prime}-\mathrm{H}_{2}\right), 2.13\left(\mathrm{~m}_{\mathrm{c}}, 1 \mathrm{H}, 4-\mathrm{H}_{\mathrm{A}}\right), 2.40$ $\left(m_{c}, 1 \mathrm{H}, 4-\mathrm{H}_{B}\right), 3.30\left(m_{c}, 1 \mathrm{H}, 4^{\prime}-\mathrm{H}\right), 3.57\left(m_{c}, 2 \mathrm{H}, 2-\mathrm{H}, 5^{\prime}-\mathrm{H}\right), 3.66\left(m_{c}, 1 \mathrm{H}, 3-\mathrm{H}\right), 3.93$ (q, J = 6.1 Hz, $1 \mathrm{H}, 7-\mathrm{H}), 4.08(\mathrm{~d}, J=9.6 \mathrm{~Hz}, 1 \mathrm{H}, \mathrm{NH}), 4.47\left(\mathrm{~d}, J=11.8 \mathrm{~Hz}, 1 \mathrm{H}, \mathrm{OCH}_{\mathrm{A}} \mathrm{Ph}\right.$ ), $4.63\left(\mathrm{~d}, J=11.8 \mathrm{~Hz}, 1 \mathrm{H}, \mathrm{OCH}_{\mathrm{B}} \mathrm{Ph}\right), 4.85\left(\mathrm{~s}_{\mathrm{br}}, 1 \mathrm{H}, 1^{\prime}-\mathrm{H}\right), 5.05\left(\mathrm{~s}, 2 \mathrm{H}, \mathrm{NC}(\mathrm{O}) \mathrm{OCH}_{2} \mathrm{Ph}\right)$, $5.42(\mathrm{dd}, J=15.5,6.1 \mathrm{~Hz}, 1 \mathrm{H}, 6-\mathrm{H}), 5.45-5.52(\mathrm{~m}, 1 \mathrm{H}, 5-\mathrm{H}), 7.16-7.39(\mathrm{~m}, 10 \mathrm{H}$, $\left.2 \times \mathrm{Ph}-\mathrm{H}_{5}\right)$.

${ }^{13} \mathrm{C}$-NMR (126 MHz, $\mathrm{CDCl}_{3}$, E-Isomer beschrieben): $\delta(\mathrm{ppm})=-4.7,-4.4\left(\mathrm{Si}\left(\mathrm{CH}_{3}\right)\right.$ ) $), 9.7$ (C-9), 15.3 (C-1), 18.1 (C-6'), $18.2\left(\mathrm{SiC}\left(\mathrm{CH}_{3}\right)_{3}\right), 25.8$ (C-3'), $25.9\left(\mathrm{SiC}\left(\underline{\mathrm{CH}}_{3}\right)_{3}\right), 29.7$ (C-2'), 31.2 (C-8), 31.6 (C-4), 52.3 (C-4'), 66.6 ( $\left.\mathrm{NC}(\mathrm{O}) \mathrm{OCH}_{2} \mathrm{Ph}\right), 68.5$ (C-5'), $71.4\left(\mathrm{OCH}_{2} \mathrm{Ph}\right), 74.6$ (C-7), 76.0 (C-2), 77.8 (C-3), 94.4 (C-1'), 125.4 (C-5), 127.5, 127.6, 128.0, 128.1, 128.3, 128.5 (4 x Ph-C $\left.\mathrm{C}_{o} 4 \times \mathrm{Ph}-\mathrm{C}_{m}, 2 \times \mathrm{Ph}-\mathrm{C}_{p}\right), 136.2,139.0\left(2 \times \mathrm{Ph}-\mathrm{C}_{i}\right), 155.8$ (NC(O)OCH$\left.{ }_{2} \mathrm{Ph}\right)$. MS (ESI): $m / z(\%)=648.4(100)[\mathrm{M}+\mathrm{Na}]^{+}$. $\mathrm{C}_{36} \mathrm{H}_{55} \mathrm{NO}_{6} \mathrm{Si}(625.91)$

ber.: 648.3691

gef.: $\quad 648.3694[\mathrm{M}+\mathrm{Na}]^{+}$(ESI-HRMS).

\subsection{2 (2S,3S,7S)-2-(Benzyloxy)-7-((tert-butyldimethylsilyl)oxy)-3-(N,N-dide-} methyl-N-benzyloxycarbonyl- $\beta$-D-forosaminyl)-non-5-en ( $\beta$-121)

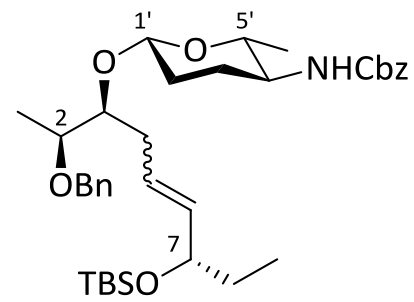

Der Alkohol $\beta$-120 (97.1 mg, $190 \mu \mathrm{mol}, 1.00$ Äq.) wurde in absolutem $\mathrm{CH}_{2} \mathrm{Cl}_{2}(12 \mathrm{~mL})$ gelöst und bei RT mit 2,6-Lutidin ( $44.2 \mu \mathrm{L}, 40.7 \mathrm{mg}, 380 \mathrm{mmol}, 2.00$ Äq.) versetzt. Die Reaktionslösung wurde auf $-10{ }^{\circ} \mathrm{C}$ gekühlt, TBSOTf $(65.4 \mu \mathrm{L}, 75.2 \mathrm{mg}, 285 \mu \mathrm{mol}$, 
1.50 Äq.) zugetropft und für $35 \mathrm{~min}$ bei $-10{ }^{\circ} \mathrm{C}$ gerührt. Durch Zugabe von $\mathrm{H}_{2} \mathrm{O}$ $(35 \mathrm{~mL})$ und langsames Erwärmen auf RT wurde die Reaktion abgebrochen. Die Phasen wurden getrennt und die wässrige Phase mit $\mathrm{CH}_{2} \mathrm{Cl}_{2}(3 \times 35 \mathrm{~mL})$ extrahiert. Die vereinigten organischen Phasen wurden über $\mathrm{MgSO}_{4}$ getrocknet und das Lösungsmittel im Vakuum entfernt. Säulenchromatographische Reinigung des Rückstands an Kieselgel (23 g, Petrolether/MTBE 5:1) lieferte die gewünschte Verbindung als Gemisch der Doppelbindungsisomere $(E)-\beta-\mathbf{1 2 1} /(Z)-\beta-121 \quad(\approx 7: 1$, 110 mg, $176 \mu \mathrm{mol}, 93 \%)$ in Form eines farblosen Öls.

$\mathbf{R}_{f}=0.33$ (Petrolether / MTBE 3:1).

UV $\left(\mathrm{CH}_{3} \mathrm{CN}\right): \lambda_{\max }(\lg \varepsilon)=252 \mathrm{~nm}$ (2.6956), 257 (3.7104).

IR: $\tilde{v}\left(\mathrm{~cm}^{-1}\right)=3326,2954,2928,2855,1698,1535,1455,1300,1247,1160,1133$, $1065,1025,985,834,774,734,696$.

${ }^{1} \mathrm{H}-\mathbf{N M R}\left(600 \mathrm{MHz}, \mathrm{CDCl}_{3}, \quad\right.$-Isomer beschrieben): $\delta(\mathrm{ppm})=-0.01,0.02(2 \times \mathrm{s}, 6 \mathrm{H}$, $\left.\mathrm{Si}\left(\mathrm{CH}_{3}\right)_{2}\right), 0.82\left(\mathrm{t}, J=7.4 \mathrm{~Hz}, 1 \mathrm{H}, 9-\mathrm{H}_{3}\right), 0.86\left(\mathrm{~s}, 9 \mathrm{H}, \mathrm{SiC}\left(\mathrm{CH}_{3}\right)_{3}\right), 1.12(\mathrm{~d}, J=6.3 \mathrm{~Hz}, 3 \mathrm{H}$, 1- $\left.\mathrm{H}_{3}\right), 1.18-1.28\left(\mathrm{~m}, 1 \mathrm{H}, 3^{\prime}-\mathrm{H}_{\mathrm{A}}\right), 1.22\left(\mathrm{~d}, J=6.1 \mathrm{~Hz}, 3 \mathrm{H}, 6^{\prime}-\mathrm{H}_{3}\right), 1.41\left(\mathrm{~m}_{\mathrm{c}}, 1 \mathrm{H}, 8-\mathrm{H}_{\mathrm{A}}\right)$, $1.48\left(\mathrm{dt}, J=13.7,6.9 \mathrm{~Hz}, 1 \mathrm{H}, 8-\mathrm{H}_{\mathrm{B}}\right), 1.54-1.63\left(\mathrm{~m}, 1 \mathrm{H}, 2^{\prime}-\mathrm{H}_{\mathrm{A}}\right), 1.80\left(\mathrm{~m}_{\mathrm{c}}, 1 \mathrm{H}, 2^{\prime}-\mathrm{H}_{\mathrm{B}}\right)$, $2.02\left(m_{c}, 1 \mathrm{H}, 3^{\prime}-\mathrm{H}_{B}\right), 2.21\left(\mathrm{~m}_{\mathrm{c}}, 1 \mathrm{H}, 4-\mathrm{H}_{\mathrm{A}}\right), 2.39\left(\mathrm{dt}, J=11.5,5.2 \mathrm{~Hz}, 1 \mathrm{H}, 4-\mathrm{H}_{\mathrm{B}}\right), 3.18$ $\left(m_{c}, 1 \mathrm{H}, 5^{\prime}-\mathrm{H}\right), 3.29-3.37\left(\mathrm{~m}, 1 \mathrm{H}, 4^{\prime}-\mathrm{H}\right), 3.52\left(\mathrm{~m}_{\mathrm{c}}, 1 \mathrm{H}, 2-\mathrm{H}\right), 3.61\left(\mathrm{~m}_{\mathrm{c}}, 1 \mathrm{H}, 3-\mathrm{H}\right), 3.91$ $(d, J=6.5 \mathrm{~Hz}, 1 \mathrm{H}, 7-\mathrm{H}), 4.37(\mathrm{~d}, J=9.1 \mathrm{~Hz}, 1 \mathrm{H}, \mathrm{NH}), 4.46\left(\mathrm{~d}, J=11.7 \mathrm{~Hz}, 1 \mathrm{H}, \mathrm{OCH}_{\mathrm{A}} P h\right)$, $4.54\left(\mathrm{dd}, J=9.2,1.2 \mathrm{~Hz}, 1 \mathrm{H}, 1^{\prime}-\mathrm{H}\right), 4.58\left(\mathrm{~d}, J=11.7 \mathrm{~Hz}, 1 \mathrm{H}, \mathrm{OCH}_{\mathrm{B}} \mathrm{Ph}\right), 5.07$ (d, $\left.J=2.9 \mathrm{~Hz}, 1 \mathrm{H}, \mathrm{NC}(\mathrm{O}) \mathrm{OCH}_{2} \mathrm{Ph}\right), 5.38(\mathrm{dd}, J=15.5,7.0 \mathrm{~Hz}, 1 \mathrm{H}, 6-\mathrm{H}), 5.54-5.63(\mathrm{~m}, 1 \mathrm{H}$, 5-H), 7.23-7.36 (m, $\left.10 \mathrm{H}, 2 \times \mathrm{Ph}-\mathrm{H}_{5}\right)$.

${ }^{13} \mathrm{C}-\mathrm{NMR}\left(126 \mathrm{MHz}, \mathrm{CDCl}_{3}\right.$, E-Isomer beschrieben): $\delta(\mathrm{ppm})=-4.7,-4.2\left(\mathrm{Si}\left(\mathrm{CH}_{3}\right)_{2}\right), 9.8$ (C-9), 15.6 (C-1), $18.2\left(\mathrm{SiC}\left(\mathrm{CH}_{3}\right)_{3}\right), 18.5\left(\mathrm{C}-6^{\prime}\right), 25.9\left(\mathrm{SiC}\left(\mathrm{CH}_{3}\right)_{3}\right), 29.8$ (C-3'), 31.0 (C-2'), 31.2 (C-8), 34.5 (C-4), 52.2 (C-4'), 66.8 ( $\left.\mathrm{NC}(\mathrm{O}) \mathrm{O} \mathrm{CH}_{2} \mathrm{Ph}\right), 71.5\left(\mathrm{OCH}_{2} \mathrm{Ph}\right), 75.1,75.1$ (C-7, C-5'), 81.1 (C-3), 102.3 (C-1'), 126.5 (C-5), 127.4, 127.6, 128.1, 128.2, 128.3, 128.5 $\left(4 \times \mathrm{Ph}-\mathrm{C}_{o}, 4 \times \mathrm{Ph}-\mathrm{C}_{m}, \quad 2 \times \mathrm{Ph}-\mathrm{C}_{p}\right), \quad 135.4$ (C-6), $138.8,138.8 \quad\left(2 \times \mathrm{Ph}-\mathrm{C}_{i}\right), \quad 155.8$ $\left(\mathrm{NC}(\mathrm{O}) \mathrm{OCH}_{2} \mathrm{Ph}\right)$.

MS (ESI): $m / z(\%)=648.4(100)[\mathrm{M}+\mathrm{Na}]^{+}, 1273.8(25)[2 \mathrm{M}+\mathrm{Na}]^{+}$. 
$\mathrm{C}_{36} \mathrm{H}_{55} \mathrm{NO}_{6} \mathrm{Si}(625.91) \quad$ ber.: 648.3691

gef.: $648.3700[\mathrm{M}+\mathrm{Na}]^{+}$(ESI-HRMS).

\subsection{3 (2S,3S,7S)-2-(Benzyloxy)-7-((tert-butyldimethylsilyl)oxy)-3-(N,N-dide-} methyl-N-benzyloxycarbonyl- $\alpha$-D-forosaminyl)-nonan ( $\alpha-122)$

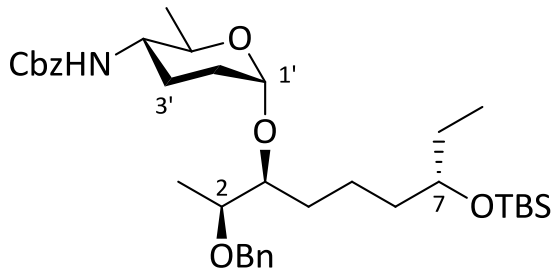

Das Glykosid $\alpha-121$ (24.0 mg, 38.3 $\mu \mathrm{mol}, 1.00$ Äq.) wurde in absolutem EtOH / $\mathrm{CH}_{2} \mathrm{Cl}_{2}$ (12:1, $13 \mathrm{~mL}$ ) gelöst und die Lösung sorgfältig entgast. Es wurde Pt/C (5\%ig, $7.50 \mathrm{mg}$, $1.92 \mu \mathrm{mol}, 5.01 \mathrm{Mol} \%)$ zugegeben und anschließend unter Rühren für $2 \mathrm{~h} \mathrm{H}_{2}$-Gas durch das Reaktionsgemisch geleitet. Es wurde über Celite filtriert und der Rückstand mit $\mathrm{CH}_{2} \mathrm{Cl}_{2}(40 \mathrm{~mL})$ gespült. Das Filtrat wurde im Vakuum eingeengt und der Rückstand säulenchromatographisch an Kieselgel (6 g, Petrolether / MTBE 5:1) gereinigt. Die Zielverbindung $\alpha-122(21.1 \mathrm{mg}, 33.6 \mu \mathrm{mol}, 88 \%)$ konnte als farbloses Öl isoliert werden.

$\mathbf{R}_{f}=0.43$ (Petrolether / MTBE 3:1).

MS (ESI): $m / z(\%)=650.4(100)[\mathrm{M}+\mathrm{Na}]^{+}$.

$\mathrm{C}_{36} \mathrm{H}_{57} \mathrm{NO}_{6} \mathrm{Si}(627.93) \quad$ ber.: 650.3847

gef.: $\quad 650.3846[\mathrm{M}+\mathrm{Na}]^{+}(\mathrm{ESI}-\mathrm{HRMS})$.

\subsection{HPLC-Reinigung von (2S,3S,7S)-2-(Benzyloxy)-7-((tert-} butyldimethylsilyl)oxy)-3-( $N, N$-didemethyl- $N$-benzyloxycarbonyl- $\alpha$-Dforosaminyl)-nonan ( $\alpha-122)$

Verbindung $\alpha$-122 (90.0 mg, $143 \mu \mathrm{mol}$ ) wurde in einem Gemisch aus $n$-Hexan / $\mathrm{CH}_{2} \mathrm{Cl}_{2}$ (1:1, $700 \mu \mathrm{L})$ gelöst. Je $150 \mu \mathrm{L}$ dieser Probenlösung (entsprechend $19.3 \mathrm{mg}$ bzw. $30.6 \mu \mathrm{mol}$ der Verbindung) wurden in das präparative HPLC-System (Säule: Chiralpak ${ }^{\circ}$ 
IA, $250 \times 20 \mathrm{~mm}$, Partikelgröße: $5 \mu \mathrm{m}$, mobile Phase: $n$-Hexan / 2-Propanol = 98:2, Fluss: $18 \mathrm{~mL}$ / min, Druck: 6.1 MPa) injiziert. Fraktioniertes Auffangen des Eluats (UVDetektor: $210 \mathrm{~nm}$ ) lieferte die enantiomerenreine Verbindung $\mathbf{\alpha - 1 2 2 . ~}$

Analytische Daten für $\alpha-122:$

HPLC (präparativ): $\quad t_{R}$ :

$8.55 \mathrm{~min}$

Fraktion: $\quad 8.20-10.8 \mathrm{~min}$

HPLC (analytisch): $\quad$ Säule: $\quad$ Chiralpak ${ }^{\circledR}$ IA

Eluens: $\quad n$-Hexan / 2-Propanol $=98: 2$

Fluss: $\quad 0.8 \mathrm{~mL} / \mathrm{min}$

$t_{\mathrm{R}}: \quad 9.92 \mathrm{~min}$.

$[\alpha]_{\mathrm{D}}^{20}=+63.2^{\circ}\left(c=0.210, \mathrm{CHCl}_{3}\right)$.

UV $\left(\mathrm{CH}_{3} \mathrm{CN}\right): \lambda_{\max }(\lg \varepsilon)=252 \mathrm{~nm}$ (2.6146), 258 (2.661).

IR: $\tilde{v}\left(\mathrm{~cm}^{-1}\right)=3316,2929,2856,1698,1533,1455,1384,1303,1248,1120,1094$, $1073,1022,987,834,772,734,696$.

${ }^{1} \mathrm{H}-\mathrm{NMR}\left(600 \mathrm{MHz}, \mathrm{CDCl}_{3}\right): \delta(\mathrm{ppm})=0.00,0.01\left(2 \times \mathrm{s}, 6 \mathrm{H}, \mathrm{Si}\left(\mathrm{CH}_{3}\right)_{2}\right), 0.83(\mathrm{t}, J=7.4 \mathrm{~Hz}$, $\left.3 \mathrm{H}, 9-\mathrm{H}_{3}\right), 0.86\left(\mathrm{~s}, 9 \mathrm{H}, \mathrm{SiC}\left(\mathrm{CH}_{3}\right)_{3}\right), 1.03\left(\mathrm{~d}, J=6.2 \mathrm{~Hz}, 3 \mathrm{H}, 6^{\prime}-\mathrm{H}_{3}\right), 1.15(\mathrm{~d}, J=6.2 \mathrm{~Hz}$, $\left.3 \mathrm{H}, 1-\mathrm{H}_{3}\right), 1.17-1.25\left(\mathrm{~m}, 1 \mathrm{H}, 5-\mathrm{H}_{\mathrm{A}}\right), 1.32-1.46\left(\mathrm{~m}, 6 \mathrm{H}, 4-\mathrm{H}_{\mathrm{A}}, 5-\mathrm{H}_{\mathrm{B}}, 6-\mathrm{H}_{2}, 8-\mathrm{H}_{2}\right), 1.54$ $\left(m_{c}, 1 \mathrm{H}, 3^{\prime}-\mathrm{H}_{\mathrm{A}}\right), 1.57-1.64\left(\mathrm{~m}, 1 \mathrm{H}, 4-\mathrm{H}_{\mathrm{B}}\right), 1.68-1.81\left(\mathrm{~m}, 3 \mathrm{H}, 2^{\prime}-\mathrm{H}_{2}, 3^{\prime}-\mathrm{H}_{\mathrm{B}}\right), 3.32\left(\mathrm{~m}_{\mathrm{c}}\right.$, $\left.1 \mathrm{H}, 4^{\prime}-\mathrm{H}\right), 3.54\left(m_{c}, 2 \mathrm{H}, 7-\mathrm{H}, 5^{\prime}-\mathrm{H}\right), 3.62\left(m_{c}, 2 \mathrm{H}, 2-\mathrm{H}, 3-\mathrm{H}\right), 4.21(\mathrm{~d}, J=9.5 \mathrm{~Hz}, 1 \mathrm{H}$, $\mathrm{NH}), 4.49\left(\mathrm{~d}, J=11.9 \mathrm{~Hz}, 1 \mathrm{H}, \mathrm{OCH}_{\mathrm{A}} \mathrm{Ph}\right), 4.61\left(\mathrm{~d}, J=11.9 \mathrm{~Hz}, 1 \mathrm{H}, \mathrm{OCH}_{\mathrm{B}} \mathrm{Ph}\right), 4.82\left(\mathrm{~s}_{\mathrm{br}}\right.$, $\left.1 \mathrm{H}, 1^{\prime}-\mathrm{H}\right), 5.06$ (s, $\left.2 \mathrm{H}, \mathrm{NC}(\mathrm{O}) \mathrm{OCH}_{2} \mathrm{Ph}\right), 7.17-7.38\left(\mathrm{~m}, 10 \mathrm{H}, 2 \times \mathrm{Ph}-\mathrm{H}_{5}\right)$.

${ }^{13} \mathrm{C}-\mathrm{NMR}\left(126 \mathrm{MHz}, \mathrm{CDCl}_{3}\right): \delta(\mathrm{ppm})=-4.5,-4.4\left(\mathrm{Si}\left(\mathrm{CH}_{3}\right)_{2}\right), 9.5$ (C-9), 14.9 (C-1), 18.1 (C-6'), $18.1\left(\mathrm{Si} \underline{C}\left(\mathrm{CH}_{3}\right)_{3}\right), 21.3$ (C-5), 25.8 (C-3'), 25.9 ( $\left.\mathrm{SiC}\left(\underline{\mathrm{CH}}_{3}\right)_{3}\right), 28.9$ (C-4), 29.8 (C-8), 29.8 (C-2'), 36.8 (C-6), 52.4 (C-4'), 66.7 (NC(O)OC $\left.\mathrm{CH}_{2} \mathrm{Ph}\right), 68.7$ (C-5'), $71.2\left(\mathrm{OCH}_{2} \mathrm{Ph}\right)$, 73.4 (C-7), 75.9 (C-2), 79.0 (C-3), 95.8 (C-1'), 127.5, 127.5 128.0, 128.1 128.3, 128.5

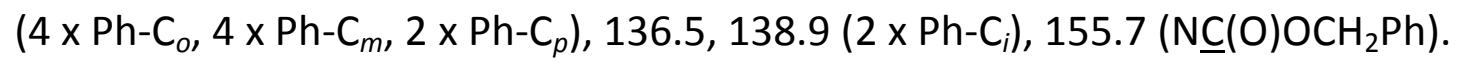


6.15 (2S,3S,7S)-2-(Benzyloxy)-7-((tert-butyldimethylsilyl)oxy)-3-(N,N-didemethyl- $N$-benzyloxycarbonyl- $\beta$-D-forosaminyl)-nonan ( $\beta$-122)

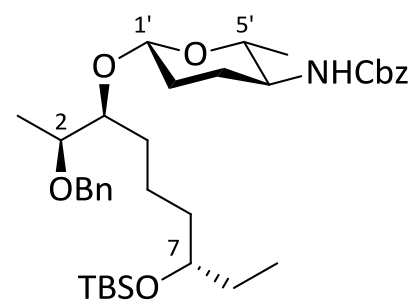

Das Glykosid $\beta$-121 (76.8 mg, $123 \mu \mathrm{mol}, 1.00$ Äq.) wurde in absolutem EtOH (30 mL) gelöst und die Lösung sorgfältig entgast. Es wurde $\mathrm{PtO}_{2}(4.20 \mathrm{mg}, 18.4 \mu \mathrm{mol}$, 15.0 Mol\%) zugegeben und anschließend unter Rühren für $2 \mathrm{~h}$ durchgehend $\mathrm{H}_{2}$-Gas durch das Reaktionsgemisch geleitet. Es wurde über Celite filtriert und der Rückstand mit $\mathrm{CH}_{2} \mathrm{Cl}_{2}(150 \mathrm{~mL})$ gespült. Das Filtrat wurde im Vakuum eingeengt und nach säulenchromatographischer Reinigung des Rückstands an Kieselgel $16 \mathrm{~g}$, Petrolether / MTBE 5:1) wurde die Zielverbindung $\beta-122$ (56.8 mg, $90.5 \mu \mathrm{mol}, 74 \%$ ) als farbloses Öl erhalten.

$\mathbf{R}_{f}=0.36$ (Petrolether / MTBE 3:1).

MS (ESI): $m / z(\%)=650.4(100)[\mathrm{M}+\mathrm{Na}]^{+}, 1277.8(64)[2 \mathrm{M}+\mathrm{Na}]^{+}$.

$\mathrm{C}_{36} \mathrm{H}_{57} \mathrm{NO}_{6} \mathrm{Si}$ (627.93) ber.: 650.3847

gef.: $\quad 650.3847[\mathrm{M}+\mathrm{Na}]^{+}(\mathrm{ESI}-\mathrm{HRMS})$.

\subsection{HPLC-Reinigung von (2S,3S,7S)-2-(Benzyloxy)-7-((tert-} butyldimethylsilyl)oxy)-3-( $N, N$-didemethyl- $N$-benzyloxycarbonyl- $\beta$-Dforosaminyl)-nonan ( $\beta$-122)

Verbindung $\beta$-122 (75.0 mg, $119 \mu \mathrm{mol})$ wurde in einem Gemisch aus $n$-Hexan / $\mathrm{CH}_{2} \mathrm{Cl}_{2}$ (1:1, $500 \mu \mathrm{L})$ gelöst. Je $50 \mu \mathrm{L}$ dieser Probenlösung (entsprechend $7.5 \mathrm{mg}$ bzw. $11.9 \mu \mathrm{mol}$ der Verbindung) wurden in das präparative HPLC-System (Säule: Chiralpak ${ }^{\bullet}$ IA, $250 \times 20 \mathrm{~mm}$, Partikelgröße: $5 \mu \mathrm{m}$, mobile Phase: $n$-Hexan / 2-Propanol = 98:2, Fluss: $18 \mathrm{~mL}$ / min, Druck: $5.9 \mathrm{MPa}$ ) injiziert. Fraktioniertes Auffangen des Eluats (UVDetektor: $210 \mathrm{~nm}$ ) lieferte die enantiomerenreine Verbindung $\beta$-122. 
Analytische Daten für $\beta-122:$

HPLC (präparativ): $\quad t_{R}$ :

Fraktion: $\quad$ 12.0-15.4 min

HPLC (analytisch):

$12.45 \mathrm{~min}$
Chiralpak ${ }^{\oplus} \mathrm{IA}$

Eluens: $\quad n$-Hexan / 2-Propanol $=98: 2$

Fluss: $\quad 0.8 \mathrm{~mL} / \mathrm{min}$

$t_{R}: \quad 14.57$ min.

$[\alpha]_{\mathrm{D}}^{20}=-13.8^{\circ}\left(c=0.305, \mathrm{CHCl}_{3}\right)$.

UV $\left(\mathrm{CH}_{3} \mathrm{CN}\right): \lambda_{\max }(\lg \varepsilon)=206 \mathrm{~nm}$ (4.1961), 258 (2.1153).

IR: $\tilde{v}\left(\mathrm{~cm}^{-1}\right)=3318,2951,2928,2855,1697,1535,1455,1299,1248,1161,1131,109$, 1064, 1025, 987, 834, 772, 735, 696.

${ }^{1} \mathrm{H}-\mathrm{NMR}\left(600 \mathrm{MHz}, \mathrm{C}_{6} \mathrm{D}_{6}\right): \delta(\mathrm{ppm})=0.09,0.14\left(2 \times \mathrm{s}, 6 \mathrm{H}, \mathrm{Si}\left(\mathrm{CH}_{3}\right)_{2}\right), 0.80\left(\mathrm{~m}_{\mathrm{c}}, 1 \mathrm{H}\right.$, $\left.3^{\prime}-\mathrm{H}_{\mathrm{A}}\right) 0.92\left(\mathrm{t}, J=7.4 \mathrm{~Hz}, 3 \mathrm{H}, 9-\mathrm{H}_{3}\right), 1.03\left(\mathrm{~s}, 9 \mathrm{H}, \mathrm{SiC}\left(\mathrm{CH}_{3}\right)_{3}\right), 1.09(\mathrm{~d}, J=6.3 \mathrm{~Hz}, 3 \mathrm{H}$, 1- $\left.\mathrm{H}_{3}\right), 1.29\left(\mathrm{~d}, J=6.0 \mathrm{~Hz}, 3 \mathrm{H}, 6^{\prime}-\mathrm{H}_{3}\right), 1.47-1.71\left(\mathrm{~m}, 10 \mathrm{H}, 4-\mathrm{H}_{2}, 5-\mathrm{H}_{\mathrm{A}}, 6-\mathrm{H}_{2}, 8-\mathrm{H}_{2}, 2^{\prime}-\mathrm{H}_{2}\right.$, 3'- $\left.\mathrm{H}_{\mathrm{B}}\right), 1.85\left(\mathrm{~m}_{\mathrm{c}}, 1 \mathrm{H}, 5-\mathrm{H}_{\mathrm{B}}\right), 2.81\left(\mathrm{~m}_{\mathrm{c}}, 1 \mathrm{H}, 5^{\prime}-\mathrm{H}\right), 3.40\left(\mathrm{~m}_{\mathrm{c}}, 1 \mathrm{H}, 4^{\prime}-\mathrm{H}\right), 3.51\left(\mathrm{~m}_{\mathrm{c}}, 1 \mathrm{H}, 2-\mathrm{H}\right)$, $3.62\left(m_{c}, 1 \mathrm{H}, 7-\mathrm{H}\right), 3.75(\mathrm{~d}, J=9.2 \mathrm{~Hz}, 1 \mathrm{H}, \mathrm{NH}), 3.85\left(\mathrm{~m}_{\mathrm{c}}, 1 \mathrm{H}, 3-\mathrm{H}\right), 4.36(\mathrm{~d}$, $\left.J=11.9 \mathrm{~Hz}, 1 \mathrm{H}, \mathrm{OCH}_{\mathrm{A}} \mathrm{Ph}\right), 4.49\left(\mathrm{~d}, J=11.9 \mathrm{~Hz}, 1 \mathrm{H}, \mathrm{OCH}_{\mathrm{B}} \mathrm{Ph}\right), 4.54(\mathrm{~d}, J=8.6 \mathrm{~Hz}, 1 \mathrm{H}$, 1'-H), 5.06 (s, $\left.2 \mathrm{H}, \mathrm{NC}(\mathrm{O}) \mathrm{OCH}_{2} \mathrm{Ph}\right), 7.00-7.34\left(\mathrm{~m}, 10 \mathrm{H}, 2 \times \mathrm{Ph}^{-\mathrm{H}_{5}}\right)$.

${ }^{13} \mathrm{C}-\mathrm{NMR}\left(126 \mathrm{MHz}, \mathrm{C}_{6} \mathrm{D}_{6}\right): \delta(\mathrm{ppm})=-4.3,-4.2\left(\mathrm{Si}\left(\mathrm{CH}_{3}\right)_{2}\right), 9.6$ (C-9), 15.6 (C-1), 18.2 $\left(\mathrm{SiC}\left(\mathrm{CH}_{3}\right)_{3}\right), 18.6$ (C-6'), 21.5 (C-5), 26.0 (C-2'), 26.0 ( $\left.\mathrm{SiC}\left(\underline{C H}_{3}\right)_{3}\right), 29.9$ (C-8), 29.9 (C-3'), 31.1 (C-4), 36.8 (C-6), 52.3 (C-4'), 66.8 ( $\left.\mathrm{NC}(\mathrm{O}) \mathrm{OCH}_{2} \mathrm{Ph}\right), 71.4\left(\mathrm{OCH}_{2} \mathrm{Ph}\right), 73.5$ (C-7), 75.1 (C-5'), 77.3 (C-2), 80.3 (C-3), 101.6 (C-1'), 127.4, 127.5, 128.0, 128.1, 128.2, 128.4

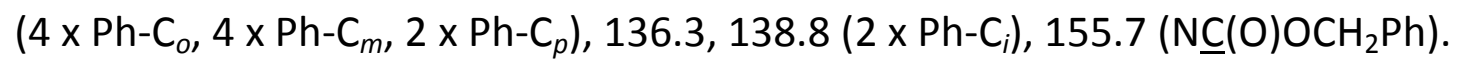




\subsection{7 (2S,3S,7S)-2-(Benzyloxy)-7-((tert-butyldimethylsilyl)oxy)-3-( $\alpha$-D-foros- aminyl)-nonan ( $\alpha-123)$}

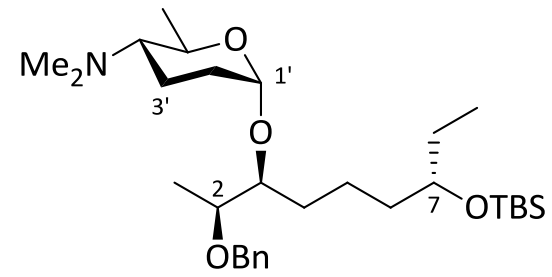

Eine Lösung von Verbindung $\alpha-122$ (57.0 mg, $90.8 \mu \mathrm{mol}, 1.00$ Äq.) und 37\%iger wässriger Formaldehydlösung ( $67.6 \mu \mathrm{L}, 73.7 \mathrm{mg}, 908 \mu \mathrm{mol}, 10.0$ Äq.) in einem Lösungsmittelgemisch aus absolutem $\mathrm{MeOH} / \mathrm{CH}_{2} \mathrm{Cl}_{2} /$ EtOAc (3:1:1, $5 \mathrm{~mL}$ ) wurde sorgfältig entgast. Es wurde Pd/C (10\%ig, 19.3 mg, 18.2 mol, 20.0 Mol\%) zugegeben und das Reaktionsgemisch in einer Parr-Apparatur bei RT für $20 \mathrm{~h}$ in einer $\mathrm{H}_{2}$ Atmosphäre (55 psi $\approx 3.8$ bar) geschüttelt. Die Suspension wurde über Celite filtriert und mit $\mathrm{CH}_{2} \mathrm{Cl}_{2}(40 \mathrm{~mL})$ gespült. Das Filtrat wurde im Vakuum eingeengt und der Rückstand säulenchromatographisch an Kieselgel (7 g, EtOAc / MeOH 98:2) gereinigt. Die Zielverbindung $\alpha-\mathbf{1 2 3}$ (39.0 mg, $74.7 \mu \mathrm{mol}, \mathbf{8 2 \%}$ ) konnte als farbloses Öl isoliert werden.

$\mathbf{R}_{f}=0.45\left(\mathrm{CH}_{2} \mathrm{Cl}_{2} / \mathrm{MeOH} 9: 1\right)$.

$[\alpha]_{\mathrm{D}}^{20}=+65.9^{\circ}\left(c=0.395, \mathrm{CHCl}_{3}\right)$.

IR: $\tilde{v}\left(\mathrm{~cm}^{-1}\right)=2923,2856,2825,2770,1454,1379,1361,1253,1233,1122,1067$, 1035, 1002, 985, 834, 772, 733, 696.

${ }^{1} \mathrm{H}-\mathrm{NMR}\left(600 \mathrm{MHz}, \mathrm{CDCl}_{3}\right): \delta(\mathrm{ppm})=0.00,0.01\left(2 \times \mathrm{s}, 6 \mathrm{H}, \mathrm{Si}\left(\mathrm{CH}_{3}\right)_{2}\right), 0.83(\mathrm{t}, J=7.4 \mathrm{~Hz}$, $\left.3 \mathrm{H}, 9-\mathrm{H}_{3}\right), 0.86\left(\mathrm{~s}, 9 \mathrm{H}, \mathrm{SiC}\left(\mathrm{CH}_{3}\right)_{3}\right), 1.11\left(\mathrm{~d}, J=6.3 \mathrm{~Hz}, 3 \mathrm{H}, 6^{\prime}-\mathrm{H}_{3}\right), 1.13-1.18(\mathrm{~m}, 1 \mathrm{H}$, $\left.5-\mathrm{H}_{\mathrm{A}}\right), 1.18\left(\mathrm{~d}, \mathrm{~J}=6.3 \mathrm{~Hz}, 3 \mathrm{H}, 1-\mathrm{H}_{3}\right), 1.34-1.47\left(\mathrm{~m}, 6 \mathrm{H}, 4-\mathrm{H}_{\mathrm{A}}, 5-\mathrm{H}_{B}, 6-\mathrm{H}_{2}, 8-\mathrm{H}_{2}\right), 1.57-$ $1.66\left(\mathrm{~m}, 3 \mathrm{H}, 4-\mathrm{H}_{B}, 2^{\prime}-\mathrm{H}_{\mathrm{A}}, 3^{\prime}-\mathrm{H}_{\mathrm{A}}\right), 1.66-1.74\left(\mathrm{~m}, 1 \mathrm{H}, 3^{\prime}-\mathrm{H}_{B}\right), 1.88\left(\mathrm{~m}, 1 \mathrm{H}, 2^{\prime}-\mathrm{H}_{B}\right), 2.15-$ $2.20\left(\mathrm{~m}, 1 \mathrm{H}, 4^{\prime}-\mathrm{H}\right), 2.18\left(\mathrm{~s}, 6 \mathrm{H}, \mathrm{N}\left(\mathrm{CH}_{3}\right)_{2}\right), 3.53\left(\mathrm{~m}_{\mathrm{c}}, 1 \mathrm{H}, 7-\mathrm{H}\right), 3.64\left(\mathrm{~m}_{\mathrm{c}}, 1 \mathrm{H}, 3-\mathrm{H}\right), 3.66-$ $3.71(\mathrm{~m}, 1 \mathrm{H}, 2-\mathrm{H}), 3.79\left(\mathrm{dq}, J=9.8,6.2 \mathrm{~Hz}, 1 \mathrm{H}, 5^{\prime}-\mathrm{H}\right), 4.52(\mathrm{~d}, J=12.1 \mathrm{~Hz}, 1 \mathrm{H}$, $\mathrm{OCH}_{\mathrm{A}} \mathrm{Ph}$ ), $4.59\left(\mathrm{~d}, J=12.1 \mathrm{~Hz}, 1 \mathrm{H}, \mathrm{OCH}_{B} \mathrm{Ph}\right), 4.78\left(\mathrm{~d}, J=2.8 \mathrm{~Hz}, 1 \mathrm{H}, 1^{\prime}-\mathrm{H}\right), 7.22-7.34$ (m, $\left.5 \mathrm{H}, \mathrm{Ph}-\mathrm{H}_{5}\right)$. 
${ }^{13} \mathrm{C}-\mathrm{NMR}\left(126 \mathrm{MHz}, \mathrm{CDCl}_{3}\right): \delta(\mathrm{ppm})=-4.3,-4.3\left(\mathrm{Si}\left(\mathrm{CH}_{3}\right)_{2}\right), 9.6(\mathrm{C}-9), 14.6(\mathrm{C}-1), 15.0$ (C-3'), $18.2\left(\mathrm{SiC}\left(\mathrm{CH}_{3}\right)_{3}\right), 18.8$ (C-6'), 22.0 (C-5), 26.0 ( $\left.\mathrm{SiC}\left(\mathrm{CH}_{3}\right)_{3}\right), 28.9$ (C-4), 29.9 (C-8), 30.5 (C-2'), 36.9 (C-6), $40.7\left(\mathrm{~N}\left(\mathrm{CH}_{3}\right)_{2}\right), 65.5$ (C-4'), 67.3 (C-5'), $71.0\left(\mathrm{OCH}_{2} \mathrm{Ph}\right), 73.4$ (C-7), 75.6 (C-2), 79.3 (C-3), 97.0 (C-1'), $127.3\left(\mathrm{Ph}-\mathrm{C}_{p}\right), 127.5$ (2x Ph-Co), 128.2 $\left(2 \times \mathrm{Ph}-\mathrm{C}_{m}\right), 138.8\left(\mathrm{Ph}-\mathrm{C}_{i}\right)$.

MS (ESI): $m / z(\%)=522.4(100)[\mathrm{M}+\mathrm{H}]^{+}, 544.4(33)[\mathrm{M}+\mathrm{Na}]^{+}$.

$\mathrm{C}_{30} \mathrm{H}_{55} \mathrm{NO}_{4} \mathrm{Si}(521.85) \quad$ ber.: 522.3973

gef.: $\quad 522.3976[\mathrm{M}+\mathrm{H}]^{+}$(ESI-HRMS).

\subsection{8 (2S,3S,7S)-2-(Benzyloxy)-7-((tert-butyldimethylsilyl)oxy)-3-( $\beta$-D-foros- aminyl)-nonan ( $\beta-123)$}

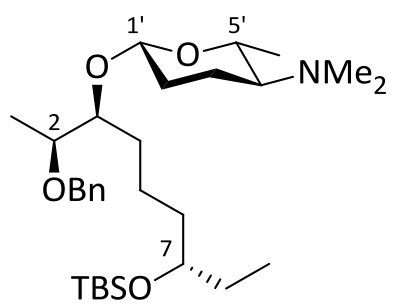

Eine Lösung von Verbindung $\beta$-122 (105 mg, $167 \mu \mathrm{mol}, 1.00$ Äq.) und 37\%iger wässriger Formaldehydlösung ( $125 \mu \mathrm{L}, 136 \mathrm{mg}, 1.67 \mathrm{mmol}, 10.0$ Äq.) in einem Lösungsmittelgemisch aus absolutem $\mathrm{MeOH} / \mathrm{CH}_{2} \mathrm{Cl}_{2} /$ EtOAc $(3: 1: 1,15 \mathrm{~mL})$ wurde sorgfältig entgast. Es wurde Pd/C (10\%ig, $35.6 \mathrm{mg}, 33.4 \mu \mathrm{mol}, 20.0 \mathrm{Mol} \%)$ zugegben und das Reaktionsgemisch in einer Parr-Apparatur bei RT für $22 \mathrm{~h}$ in einer $\mathrm{H}_{2}$ Atmosphäre ( $55 \mathrm{psi} \approx 3.8 \mathrm{bar}$ ) geschüttelt. Die Suspension wurde über Celite filtriert, mit $\mathrm{CH}_{2} \mathrm{Cl}_{2}(100 \mathrm{~mL})$ gespült und das Filtrat im Vakuum eingeengt. Säulenchromatographische Reinigung des Rückstands an Kieselgel (23 g, EtOAc / MeOH 98:2) lieferte die Titelverbindung $\beta$-123 (50.4 mg, $133 \mu \mathrm{mol}, 80 \%)$ als gelbliches Öl.

$\mathbf{R}_{f}=0.51\left(\mathrm{CH}_{2} \mathrm{Cl}_{2} / \mathrm{MeOH} 9: 1\right)$.

$[\alpha]_{\mathrm{D}}^{20}=-10.1^{\circ}\left(c=0.355, \mathrm{CHCl}_{3}\right)$.

IR: $\tilde{v}\left(\mathrm{~cm}^{-1}\right)=2928,2856,2825,2781,1454,1371,1253,1163,1123,1065,1005,988$, $834,772,733,696$. 
${ }^{1} \mathrm{H}-\mathrm{NMR}\left(600 \mathrm{MHz}, \mathrm{CDCl}_{3}\right): \delta(\mathrm{ppm})=0.01,0.02\left(2 \times \mathrm{s}, 6 \mathrm{H}, \mathrm{Si}\left(\mathrm{CH}_{3}\right)_{2}\right), 0.83(\mathrm{t}, J=7.4 \mathrm{~Hz}$, $\left.3 \mathrm{H}, 9-\mathrm{H}_{3}\right), 0.86\left(\mathrm{~s}, 9 \mathrm{H}, \mathrm{SiC}\left(\mathrm{CH}_{3}\right)_{3}\right), 1.12\left(\mathrm{~d}, J=6.3 \mathrm{~Hz}, 3 \mathrm{H}, 1-\mathrm{H}_{3}\right), 1.23(\mathrm{~d}, J=6.2 \mathrm{~Hz}$, $\left.3 \mathrm{H}, 6{ }^{\prime}-\mathrm{H}_{3}\right), 1.25-1.48\left(\mathrm{~m}, 8 \mathrm{H}, 4-\mathrm{H}_{\mathrm{A}}, 5-\mathrm{H}_{2}, 6-\mathrm{H}_{2}, 8-\mathrm{H}_{2}, 3^{\prime}-\mathrm{H}_{\mathrm{A}}\right), 1.49-1.58\left(\mathrm{~m}, 2 \mathrm{H}, 4-\mathrm{H}_{B}\right.$, $\left.2^{\prime}-H_{A}\right), 1.78\left(m_{c}, 1 \mathrm{H}, 3^{\prime}-H_{B}\right), 1.85\left(m_{c}, 1 \mathrm{H}, 2^{\prime}-H_{B}\right), 2.15-2.20\left(m, 1 \mathrm{H}, 4^{\prime}-\mathrm{H}\right), 2.20(\mathrm{~s}, 6 \mathrm{H}$, $\left.\mathrm{N}\left(\mathrm{CH}_{3}\right)_{2}\right), 3.40\left(\mathrm{dq}, J=9.4,6.1 \mathrm{~Hz}, 1 \mathrm{H}, 5^{\prime}-\mathrm{H}\right), 3.53\left(\mathrm{~m}_{\mathrm{c}}, 2 \mathrm{H}, 2-\mathrm{H}, 7-\mathrm{H}\right), 3.62\left(\mathrm{~m}_{\mathrm{c}}, 1 \mathrm{H}\right.$, 3-H), 4.46 (dd, J=9.4, 1.9 Hz, $\left.1 \mathrm{H}, 1^{\prime}-\mathrm{H}\right), 4.49$ (d, J=11.9 Hz, $1 \mathrm{H}, \mathrm{OCH}_{\mathrm{A}} \mathrm{Ph}$ ), 4.59 (d, $\left.J=11.9 \mathrm{~Hz}, 1 \mathrm{H}, \mathrm{OCH}_{\mathrm{B}} \mathrm{Ph}\right), 7.23-7.34\left(\mathrm{~m}, 5 \mathrm{H}, \mathrm{Ph}-\mathrm{H}_{5}\right)$.

${ }^{13} \mathrm{C}-\mathrm{NMR}\left(126 \mathrm{MHz}, \mathrm{CDCl}_{3}\right): \delta(\mathrm{ppm})=-4.3,-4.2\left(\mathrm{Si}\left(\mathrm{CH}_{3}\right)_{2}\right), 9.6$ (C-9), $15.5(\mathrm{C}-1), 18.2$ $\left(\mathrm{SiC}\left(\mathrm{CH}_{3}\right)_{3}\right), 18.5$ (C-3'), 19.1 (C-6'), 21.6 (C-5), $26.0\left(\mathrm{SiC}\left(\underline{\mathrm{CH}}_{3}\right)_{3}\right), 29.9$ (C-4), 31.0 (C-2'), 31.5 (C-8), 36.9 (C-6), $40.7\left(\mathrm{~N}\left(\mathrm{CH}_{3}\right)_{2}\right), 65.0$ (C-4'), $71.4\left(\mathrm{OCH}_{2} \mathrm{Ph}\right), 73.5(\mathrm{C}-2), 73.6\left(\mathrm{C}-5^{\prime}\right)$, 77.1 (C-7), 80.0 (C-3), 101.9 (C-1'), $127.3\left(\mathrm{Ph}-\mathrm{C}_{p}\right), 127.5$ (2x Ph-C $), 128.2\left(2 \times \mathrm{Ph}-\mathrm{C}_{m}\right)$, $138.9\left(\mathrm{Ph}-\mathrm{C}_{i}\right)$.

MS (ESI): $m / z(\%)=522.4(100)[\mathrm{M}+\mathrm{H}]^{+}, 1043.8(45)[2 \mathrm{M}+\mathrm{H}]^{+}, 1065.8(25)[2 \mathrm{M}+\mathrm{Na}]^{+}$, $544.4(18)[\mathrm{M}+\mathrm{Na}]^{+}$.

$\mathrm{C}_{30} \mathrm{H}_{55} \mathrm{NO}_{4} \mathrm{Si}(521.85) \quad$ ber.: 522.3973

gef.: $\quad 522.3972[\mathrm{M}+\mathrm{H}]^{+}(\mathrm{ESI}-\mathrm{HRMS})$.

\section{$6.19(2 S, 3 S, 7 S)-7-(($ tert-Butyldimethylsilyl)oxy)-3-( $\alpha$-D-forosaminyl)-nonan- 2-ol ( $\alpha-103)$}

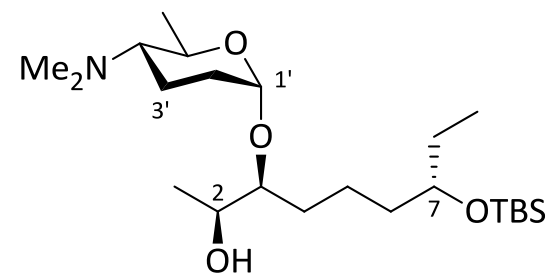

Es wurde $\mathrm{NH}_{3}$-Gas $(30 \mathrm{~mL})$ kondensiert und bei $-78^{\circ} \mathrm{C}$ mit Lithium-Granulat $(28.0 \mathrm{mg}$, $4.03 \mathrm{mmol}, 62.8$ Äq.) versetzt. Die tiefblaue Lösung wurde tropfenweise mit dem benzylgeschützten Alkohol $\alpha-123$ (33.5 mg, $64.2 \mu \mathrm{mol}, 1.00$ Äq.), gelöst in absolutem THF $(1.5 \mathrm{~mL})$, versetzt und anschließend für $1 \mathrm{~h}$ bei $-78^{\circ} \mathrm{C}$ gerührt. Die Reaktion wurde durch langsame Zugabe von $\mathrm{MeOH}(3 \mathrm{~mL}$ ) beendet (Entfärbung) und überschüssiges $\mathrm{NH}_{3}$ durch langsames Erwärmen auf RT verdampft. Das Lösungsmittel wurde im Vakuum entfernt und der Rückstand durch säulenchromatographische 
Reinigung an Kieselgel (23 g, EtOAc / MeOH 99:1) gereinigt. Die Zielverbindung $\alpha-103$ (25.1 mg, $58.1 \mu \mathrm{mol}, 91 \%$ ) wurde als farbloses Öl erhalten.

$\mathbf{R}_{f}=0.25\left(\mathrm{CH}_{2} \mathrm{Cl}_{2} / \mathrm{MeOH} 9: 1\right)$.

$[\alpha]_{\mathrm{D}}^{20}=+39.9^{\circ}\left(c=0.380, \mathrm{CHCl}_{3}\right)$.

IR: $\tilde{v}\left(\mathrm{~cm}^{-1}\right)=3429,2929,2856,2826,2781,1461,1379,1363,1254,1233,1119$, 1066, 1049, 1035, 988, 834, 772.

${ }^{1} \mathrm{H}-\mathrm{NMR}\left(600 \mathrm{MHz}, \mathrm{CDCl}_{3}\right): \delta(\mathrm{ppm})=0.01\left(2 \times \mathrm{s}, 6 \mathrm{H}, \mathrm{Si}\left(\mathrm{CH}_{3}\right)_{2}\right), 0.83(\mathrm{t}, J=7.4 \mathrm{~Hz}, 3 \mathrm{H}$, 9- $\left.\mathrm{H}_{3}\right), 0.86\left(\mathrm{~s}, 9 \mathrm{H}, \mathrm{SiC}\left(\mathrm{CH}_{3}\right)_{3}\right), 1.12\left(\mathrm{~d}, J=6.4 \mathrm{~Hz}, 3 \mathrm{H}, 6^{\prime}-\mathrm{H}_{3}\right), 1.22(\mathrm{~d}, J=6.2 \mathrm{~Hz}, 3 \mathrm{H}$, $\left.1-\mathrm{H}_{3}\right), 1.22-1.26\left(\mathrm{~m}, 1 \mathrm{H}, 5-\mathrm{H}_{A}\right), 1.33-1.52\left(\mathrm{~m}, 7 \mathrm{H}, 4-\mathrm{H}_{2}, 5-\mathrm{H}_{\mathrm{B}}, 6-\mathrm{H}_{2}, 8-\mathrm{H}_{2}\right), 1.63(\mathrm{dt}$, $\left.J=7.1,3.5 \mathrm{~Hz}, 1 \mathrm{H}, 3^{\prime}-\mathrm{H}_{\mathrm{A}}\right), 1.66-1.74\left(\mathrm{~m}, 2 \mathrm{H}, 2^{\prime}-\mathrm{H}_{\mathrm{A}}, 3^{\prime}-\mathrm{H}_{\mathrm{B}}\right), 1.85-1.90\left(\mathrm{~m}, 1 \mathrm{H}, 2^{\prime}-\mathrm{H}_{B}\right)$, 2.18-2.25 (m, $\left.1 \mathrm{H}, 4^{\prime}-\mathrm{H}\right), 2.22\left(\mathrm{~s}, 6 \mathrm{H}, \mathrm{N}\left(\mathrm{CH}_{3}\right)_{2}\right), 3.27\left(\mathrm{~m}_{\mathrm{c}}, 1 \mathrm{H}, 3-\mathrm{H}\right), 3.54\left(\mathrm{~m}_{\mathrm{c}}, 1 \mathrm{H}, 7-\mathrm{H}\right)$, $3.67\left(m_{c}, 1 \mathrm{H}, 2-\mathrm{H}\right), 3.73\left(\mathrm{~s}_{\mathrm{br}}, 1 \mathrm{H}, \mathrm{OH}\right), 3.93\left(\mathrm{dq}, J=9.8,6.2 \mathrm{~Hz}, 1 \mathrm{H}, 5^{\prime}-\mathrm{H}\right), 4.79\left(\mathrm{~s}_{\mathrm{br}}\right.$, $\left.1 \mathrm{H}, 1^{\prime}-\mathrm{H}\right)$.

$\left.{ }^{13} \mathrm{C}-\mathrm{NMR}\left(126 \mathrm{MHz}, \mathrm{CDCl}_{3}\right): \delta(\mathrm{ppm})=-4.4,-4.3\left(\mathrm{Si}_{\left(\mathrm{CH}_{3}\right)}\right)_{2}\right), 9.6$ (C-9), 14.9 (C-3'), 18.2 $\left.\left(\mathrm{SiC}(\underline{\mathrm{CH}})_{3}\right)_{3}\right), 19.0\left(\mathrm{C}-6^{\prime}\right), 19.2(\mathrm{C}-1), 21.2(\mathrm{C}-5), 26.0\left(\mathrm{SiC}\left(\mathrm{CH}_{3}\right)_{3}\right), 29.9$ (C-4), $30.5\left(\mathrm{C}-2^{\prime}\right)$,

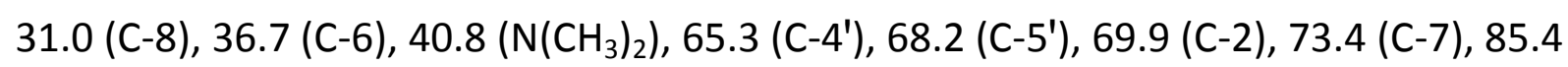
(C-3), $97.2\left(\mathrm{C}-1^{\prime}\right)$.

MS (ESI): $m / z(\%)=432.4(100)[\mathrm{M}+\mathrm{H}]^{+}, 885.7(43)[2 \mathrm{M}+\mathrm{H}]^{+}, 454.3(23)[\mathrm{M}+\mathrm{Na}]^{+}$.

$\mathrm{C}_{23} \mathrm{H}_{49} \mathrm{NO}_{4} \mathrm{Si}(431.73)$

ber.: 432.3504

gef.: $432.3504[\mathrm{M}+\mathrm{H}]^{+}$(ESI-HRMS). 


\section{$6.20(2 S, 3 S, 7 S)-7-(($ tert-Butyldimethylsilyl)oxy)-3-( $\beta$-D-forosaminyl)-nonan- 2-ol ( $\beta$-103)}

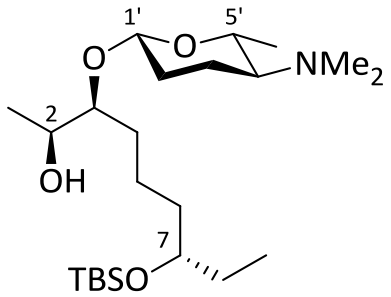

Es wurde $\mathrm{NH}_{3}$-Gas $(20 \mathrm{~mL})$ kondensiert und bei $-78{ }^{\circ} \mathrm{C}$ mit Lithium-Granulat $(49.0 \mathrm{mg}$, $7.06 \mathrm{mmol}$, 53.1 Äq.) versetzt. Die tiefblaue Lösung wurde tropfenweise mit dem benzylgeschützten Alkohol $\beta-123$ (69.3 mg, 133 mol, 1.00 Äq.), gelöst in absolutem THF $(1.5 \mathrm{~mL})$, versetzt und anschließend für $1 \mathrm{~h}$ bei $-78{ }^{\circ} \mathrm{C}$ gerührt. Die Reaktion wurde durch langsame Zugabe von $\mathrm{MeOH}(5 \mathrm{~mL}$ ) beendet (Entfärbung) und überschüssiges $\mathrm{NH}_{3}$ durch langsames Erwärmen auf RT verdampft. Das Lösungsmittel wurde im Vakuum entfernt und der Rückstand säulenchromatographisch an Kieselgel

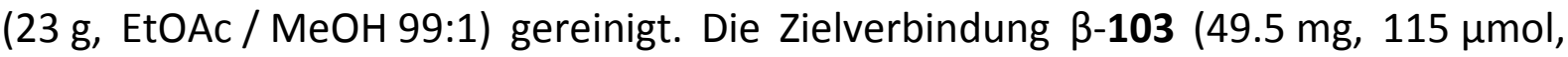
$86 \%)$ wurde als farbloses Öl erhalten.

$\mathbf{R}_{f}=0.16\left(\mathrm{CH}_{2} \mathrm{Cl}_{2} / \mathrm{MeOH} 9: 1\right)$.

$[\alpha]_{\mathrm{D}}^{20}=+3.7^{\circ}\left(c=0.375, \mathrm{CHCl}_{3}\right)$.

IR: $\tilde{v}\left(\mathrm{~cm}^{-1}\right)=3435,2928,2856,2828,2781,1461,1370,1253,1163,1123,1065$, $1005,988,834,772$.

${ }^{1} \mathrm{H}-\mathrm{NMR}\left(600 \mathrm{MHz}, \mathrm{CDCl}_{3}\right): \delta(\mathrm{ppm})=0.01\left(\mathrm{~s}, 6 \mathrm{H}, \mathrm{Si}\left(\mathrm{CH}_{3}\right)_{2}\right), 0.83(\mathrm{t}, J=7.4 \mathrm{~Hz}, 3 \mathrm{H}$, 9- $\left.\mathrm{H}_{3}\right), 0.85\left(\mathrm{~s}, 9 \mathrm{H}, \mathrm{SiC}\left(\mathrm{CH}_{3}\right)_{3}\right), 1.13\left(\mathrm{~d}, J=6.4 \mathrm{~Hz}, 3 \mathrm{H}, 1-\mathrm{H}_{3}\right), 1.25(\mathrm{~d}, J=6.2 \mathrm{~Hz}, 3 \mathrm{H}$, 6'- $\left.\mathrm{H}_{3}\right), 1.29-1.36\left(\mathrm{~m}, 2 \mathrm{H}, 5-\mathrm{H}_{\mathrm{A}}, 6-\mathrm{H}_{\mathrm{A}}\right), 1.37-1.48\left(\mathrm{~m}, 5 \mathrm{H}, 4-\mathrm{H}_{2}, 5-\mathrm{H}_{B}, 6-\mathrm{H}_{B}, 3-\mathrm{H}_{\mathrm{A}}\right), 1.48-$ $1.57\left(\mathrm{~m}, 3 \mathrm{H}, 8-\mathrm{H}_{2}, 2^{\prime}-\mathrm{H}_{\mathrm{A}}\right), 1.83$ (ddd, $J=12.6,7.1,3.6 \mathrm{~Hz}, 1 \mathrm{H}, 3^{\prime}-\mathrm{H}_{\mathrm{B}}$ ), 1.88 (ddd, $\left.J=12.6,5.6,3.4 \mathrm{~Hz}, 1 \mathrm{H}, 2^{\prime}-\mathrm{H}_{\mathrm{B}}\right), 2.16-2.22\left(\mathrm{~m}, 1 \mathrm{H}, 4^{\prime}-\mathrm{H}\right), 2.20\left(\mathrm{~s}, 6 \mathrm{H}, \mathrm{N}\left(\mathrm{CH}_{3}\right)_{2}\right), 2.89$ $\left(s_{b r}, 1 \mathrm{H}, \mathrm{OH}\right), 3.38\left(m_{c}, 1 \mathrm{H}, 3-\mathrm{H}\right), 3.48(\mathrm{dq}, J=9.4,6.2 \mathrm{~Hz}, 1 \mathrm{H}, 5 '-\mathrm{H}), 3.54$ (quint, $J=6.2 \mathrm{~Hz}, 1 \mathrm{H}, 7-\mathrm{H}$ ), 3.64 (quint, $J=6.4 \mathrm{~Hz}, 1 \mathrm{H}, 2-\mathrm{H}$ ), 4.48 (dd, J = 9.6, $2.0 \mathrm{~Hz}, 1 \mathrm{H}$, $\left.1^{\prime}-\mathrm{H}\right)$.

${ }^{13} \mathrm{C}-\mathrm{NMR}\left(126 \mathrm{MHz}, \mathrm{CDCl}_{3}\right): \delta(\mathrm{ppm})=-4.3,-4.3\left(\mathrm{Si}\left(\mathrm{CH}_{3}\right)_{2}\right), 9.6(\mathrm{C}-9), 18.2\left(\mathrm{SiC}\left(\mathrm{CH}_{3}\right)_{3}\right)$, 18.5 (C-3'), 19.1 (C-6'), 19.4 (C-1), 21.4 (C-5), 26.0 ( $\left.\mathrm{SiC}\left(\mathrm{CH}_{3}\right)_{3}\right), 29.9$ (C-4), 31.3 (C-2'), 
31.4 (C-8), 36.8 (C-6), $40.7\left(\mathrm{~N}\left(\mathrm{CH}_{3}\right)_{2}\right), 64.8$ (C-4'), 69.1 (C-2), 73.4 (C-7), 74.0 (C-5'), 83.7 (C-3), $100.7\left(C-1^{\prime}\right)$.

MS (ESI): $m / z(\%)=432.4(100)[\mathrm{M}+\mathrm{H}]^{+}, 885.7(23)[2 \mathrm{M}+\mathrm{H}]^{+}$.

$\mathrm{C}_{23} \mathrm{H}_{49} \mathrm{NO}_{4} \mathrm{Si}(431.73)$ ber.: 432.3504 gef.: $432.3507[\mathrm{M}+\mathrm{H}]^{+}$(ESI-HRMS). 


\section{G. ANHANG}

\section{Abkürzungen und Akronyme}

\begin{tabular}{|c|c|}
\hline$\AA$ & Ångström $\left(10^{-10} \mathrm{~m}\right)$ \\
\hline Ac & Acetyl \\
\hline Äq. & Äquivalente \\
\hline aq. & wäßrig \\
\hline atm & Atmosphäre \\
\hline ber. & berechnet \\
\hline $\mathrm{Bn}$ & Benzyl \\
\hline $\mathrm{Bu}$ & Butyl \\
\hline bzw. & beziehungsweise \\
\hline c & Konzentration \\
\hline $\mathrm{Cbz}$ & Benzyloxycarbonyl \\
\hline COSY & correlated spectroscopy \\
\hline$d$ & Tag \\
\hline DBU & 1,8-Diazabicyclo[5.4.0]-undec-7-en \\
\hline $\mathrm{DC}$ & Dünnschichtchromatographie \\
\hline $\mathrm{DCl}$ & Direkte Chemische Ionisation \\
\hline DDT & Dichlordiphenyltrichlorethan \\
\hline d. h. & das heißt \\
\hline DIBAL & Diisobutylaluminiumhydrid \\
\hline DMAP & 4-N,N-Dimethylaminopyridin \\
\hline DMF & $N, N$-Dimethylformamid \\
\hline DMI & Demethylierungsinhibitoren \\
\hline DMP & Dess-Martin-Periodinan \\
\hline DMSO & Dimethylsulfoxid \\
\hline d.s. & Diastereoselektivität \\
\hline EA & Evans-Auxiliar \\
\hline$e e$ & Enantiomerenüberschuss \\
\hline $\mathrm{El}$ & Elektronenstoß-Ionisation \\
\hline
\end{tabular}


ESI

Et

EtOAc

$\mathrm{Et}_{2} \mathrm{O}$

gef.

ges.

$\mathrm{h}$

HBK

$\mathrm{HMBC}$

HOAC

HPLC

HRMS

HSQC

$\mathrm{Hz}$

i

Inj.

iPr

IR

kat.

KHMDS

konz.

$\mathrm{L}$

$\mathrm{LC}_{50}$

$\mathrm{LD}_{50}$

Lsg.

$m$

M

$\mathrm{Me}$

$\mathrm{MeOH}$

$\min$

Ms
Elektrospray-Ionisation

Ethyl

Essigsäureethylester

Diethylether

gefunden

gesättigt

Stunde

Herrmann-Beller-Katalysator

heteronuclear multiple-bond correlation

Eisessig

high performance (pressure) liquid chromatography

high resolution mass spectrometry

heteronuclear single-quantum correlation

Hertz

ipso

Injektion

iso-Propyl

Infrarot-Spektroskopie

katalytische Mengen

Kalium-bis-(trimethylsilyl)amid

konzentriert

Ligand

lethal concentration; Toxinkonzentration in Luft, Wasser oder Boden, bei der $50 \%$ der Versuchstiere sterben

lethal dose; verabreichte Einzeldosis, bei der $50 \%$ der

Versuchstiere sterben

Lösung

meta

Molar

Methyl

Methanol

Minute

Mesyl (Methansulfonyl) 


\begin{tabular}{|c|c|}
\hline MS & Molsieb \\
\hline MS & Massenspektrometrie \\
\hline $\mathrm{MsCl}$ & Methansulfonsäurechlorid \\
\hline MTBE & Methyl-tert-butylether \\
\hline$n$ & normal, unverzweigt \\
\hline NBS & $N$-Bromsuccinimid \\
\hline NCS & $N$-Chlorsuccinimid \\
\hline NMR & nuclear magnetic resonance \\
\hline NOE & nuclear Overhauser effect \\
\hline NOESY & NOE-Spektroskopie \\
\hline $\mathrm{Nu}$ & Nucleophil \\
\hline 0 & ortho \\
\hline$p$ & para \\
\hline PG & protecting group (Schutzgruppe) \\
\hline $\mathrm{Ph}$ & Phenyl \\
\hline PMB & p-Methoxybenzyl \\
\hline ppm & parts per million \\
\hline PS & Polymer supportet (Polymer-gebunden) \\
\hline Pv & Pivaloyl \\
\hline quant. & quantitativ \\
\hline $\mathrm{R}$ & Organischer Rest \\
\hline $\mathrm{R}_{f}$ & Retentionswert für DC \\
\hline rac & racemisch \\
\hline Rham & 2,3,4-Tri-O-methyl-L-rhamnopyranosyl \\
\hline RT & Raumtemperatur \\
\hline Sdp. & Siedepunkt \\
\hline Smp. & Schmelzpunkt \\
\hline $\mathrm{T}$ & Temperatur \\
\hline$t$ & tertiär \\
\hline$t_{\mathrm{R}}$ & Retentionszeit für HPLC \\
\hline TBDPS & tert-Butyldiphenylsilyl \\
\hline TBS & tert-Butyldimethylsilyl \\
\hline
\end{tabular}




$\begin{array}{ll}\text { tert } & \text { tertiär } \\ \text { TES } & \text { Triethylsilyl } \\ \text { Tf } & \text { Trifluormethylsulfonyl } \\ \text { TfO }_{2} & \text { Trifluormethansulfonsäureanhydrid } \\ \text { TfOH } & \text { Trifluormethansulfonsäure } \\ \text { THF } & \text { Tetrahydrofuran } \\ \text { THP } & \text { Tetrahydro-2-pyranyl } \\ \text { TMI } & \text { 5,6,7-Trimethoxy-1H-indol } \\ \text { TMS } & \text { Trimethylsilyl } \\ \text { Ts } & \text { Tosyl (Toluolsulfonyl) } \\ \text { TsCl } & \text { Toluolsulfonsäurechlorid } \\ \text { TsOH } & \text { Toluolsulfonsäure } \\ \text { UV } & \text { Ultraviolett-Spektroskopie } \\ \text { X } & \text { Halogenid } \\ \text { z. B. } & \text { zum Beispiel } \\ \text { z. T. } & \text { zum Teil } \\ \text { 2,4-D } & \text { 2,4-Dichlorphenoxyessigsäure } \\ & \end{array}$




\section{Literatur}

J. Stetter, F. Lieb, Angew. Chem. 2000, 112, 1792-1812; Angew. Chem. Int. Ed. 2000, 39, 1724-11744.

W. Schwab, Pharm. uns. Zeit 2000, 2, 107-114.

a) C. Fest, K. J. Schmidt; Chemie der Pflanzenschutz- und Schädlingsbekämpfungsmittel, Bd. 1 (Hrsg.: R. Wegler), Springer, Berlin, 1970, S. 246-453;

b) M. Eto, Organophosphorous Pesticides: Organic and Biological Chemistry, CRC, Cleveland, Ohio, 1974.

a) K. Mellanby, The DDT Story, The British Crop Protection Council, Farnham, Surrey, Großbritannien, 1992; b) R. G. Beatty, The DDT Myth, John Day, New York, 1973; c) H. Röchling; Chemie der Pflanzenschutz- und Schädlingsbekämpfungsmittel, Bd. 1 (Hrsg.: R. Wegler), Springer, Berlin, 1970, S. 121-129.

R. Wegler, L. Eue; Chemie der Pflanzenschutz- und Schädlingsbekämpfungsmittel, Bd. 5 (Hrsg.: R. Wegler), Springer, Berlin, 1977, S. 180-191.

W. Krämer; Chemie der Pflanzenschutz- und Schädlingsbekämpfungsmittel (Hrsg.: K. H. Büschel), Springer, Stuttgart, 1977, S. 137.

M. Perugini, M. Cavaliere, A. Giammarino, P. Mazzone, V. Olivieri, M. Amorena, Chemosphere 2004, 57, 391-400.

M. Zumbado, M. Goethals, E. E. Álvarez-León, O. P. Luzardo, F. Cabrera, L. Serra-Majem, L. Dominguez-Boada, Sci. Total Environ. 2005, 339, 49-62.

R. Carson, Silent Spring, Houghton Mifflin, Boston, 1962.

Verordnung (EG) Nr. 1107/2009 des Europäischen Parlaments und des Rates vom 21. Oktober 2009 über das Inverkehrbringen von Pflanzenschutzmitteln und zur Aufhebung der Richtlinien 79/117/EWG und 91/414/EWG des Rates. Pressemitteilung Nr. 169 des Bundesministeriums für Ernährung, Landwirtschaft und Verbraucherschutz (31.08.2011). M. Henningsen, Chem. uns. Zeit 2003, 27, 98-111. 
Bundesministerium für Ernährung, Landwirtschaft und Verbraucherschutz (Hrsg.): Statistisches Jahrbuch über Ernährung, Landwirtschaft und Forsten der Bundesrepublik Deutschland Jahrgang 2010, NW-Verlag, 2011.

H. Sauter, W. Steglich, T. Anke, Angew. Chem. 1999, 111, 1416-1438.

T. Seitz, M. G. Hoffmann, H. Krähmer, Chem. uns. Zeit 2003, 37, 112-126.

J. Davies, J. C. Casaley, Pestic. Sci. 1999, 55, 1043-1058.

M. Beckmann, K.-J. Haack, Chem. uns. Zeit 2003, 37, 88-97.

Dieses Kapitel wurde in Anlehnung an folgende Arbeiten verfasst: a) G. Brasche, Dissertation, Göttingen 2005; b) C. Stadler, Dissertation, Göttingen 2006; c) A. Grube, Dissertation, Göttingen 2007, N. Böhnke, Dissertation, Göttingen 2007.

Eine hervorragende Übersicht liefert: H. A. Kirst, J. Antibiot. 2010, 63, 101-111. F. P. Mertz, R. C. Yao, Int. J. Syst. Bacteriol. 1990, 40, 34-39.

M. Bertasso, M. Holzenkämpfer, A. Zeeck, E. Stackebrandt, W. Beil, H.-P. Fiedler, J. Antibiot. 2003, 56, 365-371.

H. Seto, H. Yonehara, S. Aizawa, H. Akutsu, J. Clardy, E. Arnold, M. Tanabe, S. Urano, Chem. Abstr. 1980, 92, 211459u.

P. Lewer, D. R. Hahn, L. L. Karr, D. O. Duebelbeis, J. R. Gilbert, G. D. Crouse, T. Worden, T. C. Sparks, P. McKamey, R. Edwards, P. R. Graupner, Bioorg. Med. Chem. 2009, 17, 4185-4196.

T. Perry, J. A. McKenzie, P. Batterham, Insect. Biochem. Molec. Biol. 2007, 37, 184-188.

G. B. Watson, Pest. Biochem. Physiol. 2009, 71, 20-28.

N. Orr, A. J. Shaffner, K. Richey, G. D. Crouse, Pest. Biochem. Physiol. 2009, 95, $1-5$.

T. C. Sparks, J. J. Sheets, J. R. Skomp, T. V. Worden, M. B. Hertlein, D. Bellows, S. Thibault, L. Wally, Proc. Beltwide Cotton Production Conf. 1997, 1259-1264. G. D. Thompson, R. Dutton, T. C. Sparks, Pest. Manag. Sci. 2000, 56, 696-702. 
T. C. Sparks, G. D. Crouse, G. Durst, Pest, Manag. Sci. 2001, 57, 896-905. BienSchV 1992; Bienenschutzverordnung vom 22. Juli 1992 (BGBI. I S.1420). E. I. Rabea, H. M. Nasr, M. E. I. Badaway, Arch. Environ. Contam. Toxicol. 2010, $58,722-732$.

M. Miles, Bull. Insectol. 2003, 56, 119-124.

S. Liu, Q. X. Li, Chemosphere 2004, 56, 1121-1127.

a) M. D. K. Markussen, M. Kristensen, Pest. Manag. Sci. 2012, 68, 75-82; b) T. Shono, J. G. Scott, Pestic. Biochem. Physiol. 2003, 75, 1-7; c) H. P. Young, W. B. Bailey, R. M. Roe, T. Iwasa, T. C. Sparks, Proc. Beltwide Cotton Production Conf. 2001, S. 1167-1171; d) C. F. Wyuss, H. P. Young, J. Shukla, R. M. Roe, Crop. Protect. 2003, 22, 307-314; e) W. D. Bailey, H. P. Young, R. M. Roe, Proc. Beltwide Cotton Production Conf. 1999, S. 1221-1224.

a) J. K. Moulton, D. A. Pepper, T. J. Dennehy, Proc. Beltwide Cotton Production Conf. 1999, S. 884-889; b) J. K. Moulton, D. A. Pepper, T. J. Dennehy, Pest Manag. Sci. 2000, 56, 842-848; c) J.-Z. Zhao, Y.-X. Li, H. L. Collins, L. GusukumaMinuto, R. F. L. Mau, G. D. Thompson, A. M. Shelton, J. Econ. Entomol. 2002, 95, 430-436; d) A. H. Sayyed, D. Omar, D. J. Wright, Pest. Manag. Sci. 2004, 60, 827-832.

T. C. Sparks, P. B. Anzeveno, J. G. Martynow, J. Gifford, M. B. Hertlein, T. V. Worden, H. A. Kirst, Pest. Biochem. Physiol. 2000, 67, 103-110.

L. C. Creemer, H. A. Kirst, J. W. Paschal, J. Antibiot. 1998, 51, 795-800

G. D. Crouse, T. C. Sparks, J. Schoonover, J. Gifford, J. Dripps, T. Bruce, L. J. Larson, J. Garlich, C. Hatton, R. L. Hill, T. V. Worden, J. G. Martynow, Pest, Manag. Sci. 2001, 57, 177-185.

J. E. Dripps, B. Olson, T. C. Sparks, G. D. Crouse, Plant Health Progress 2008, (22. August 2008).

L. Besard, V. Mommaerts, G. Abdu-Alla, G. Smagghe, Pest. Manag. Sci. 2011, $67,541-547$. 
41

J. Daeuble, T. C. Sparks, P. Johnson, P. R. Graupner, Bioorg. Med. Chem. 2009, 17, 4197-4205.

H. J. Kim, M. W. Ruszczycky, S. Choi, Y. Liu, H. Liu, Nature 2011, 473, 109-112.

H. J. Kim, J. A. White-Phillip, Y. Ogasawara, N. Shin, E. A. Isiorho, H.-W. Liu, J. Am. Chem. Soc. 2010, 132, 2901-2903.

Für synthetische Beispiele siehe: C. E. Aroyan, A. Dermenci, S. J. Miller, Tetrahedron 2009, 65, 4069-4084.

D. A. Evans, W. C. Black, J. Am. Chem. Soc. 1993, 115, 4497-4513.

a) L. A. Paquette, Z. Gao, Z. Ni, G. F. Smith, J. Am. Chem. Soc. 1998, 120, 2543-2552; b) L. A. Paquette, I. Collado, M. Purdie, J. Am. Chem. Soc. 1998, 120, 2553-2562; c) L. A. Paquette, Z. Gao, Z. Ni, G. F. Smith, Tetrahedron Lett. 1997, 38, 1271-1274.

a) D. J. Mergott, S. A. Frank, W. R. Roush, Org. Lett. 2002, 4, 3157-3160; b) D. J. Mergott, S. A. Frank, W. R. Roush, Proc. Natl. Acad. Sci. U.S.A. 2004, 101, 11955-11959.

Übersichten zu Dominoreaktionen bieten: a) L. F. Tietze, Chem. Rev. 1996, 96, 115-136; b) L. F. Tietze, U. Beifuss, Angew. Chem. 1993, 105, 137-170; Angew. Chem. Int. Ed. Engl. 1993, 32, 131-163.

S. Goldstein, P. Vannes, C. Houge, A. M. Frisque-Hesbain, C. Wiaux-Zamar, L. Ghosez, G. Germain, J. P. Declercq, M. Van Meerssche, J. M. Arrieta, J. Am. Chem. Soc. 1981, 103, 4616-4618.

L. F. Tietze, G. Brasche, A. Grube, N. Böhnke, C. Stadler, Chem. Eur. J. 2007, 13, 8543-8563.

D. S. Breslow, E. Baumgarten, C. R. Hauser, J. Am. Chem. Soc. 1944, 66, 12801288.

D. N. Harpp, L. Q. Bao, C. J. Black, J. G. Gleason, R. A. Smith, J. Org. Chem. 1975, $40,3420-3427$. 
I. Flemming, Grenzorbitale und Reaktionen organischer Verbindungen, WileyVCH, Weinheim, 1990.

B. B. Snider, B. A. McCarthy, Tetrahedron 1993, 49, 9447-9452.

a) P. J. Kocieński, Protecting Groups, 3rd Ed., Thieme, Stuttgart, New York, 2003; b) T. W. Greene, P. G. M. Wuts, Protective Groups in Organic Chemistry, John Wiley \& Sons Inc, New York, 1999.

Zur Darstellung des Isoharnstoffs siehe: E. Schmidt, F. Moosmüller, Justus Liebigs Ann. Chem. 1955, 597, 235-240.

G. Capozzi, C. Ciampi, G. Delogu, S. Menichetti, C. Nativi, J. Org. Chem. 2001, $66,8787-8792$.

R. M. Giuliano, S. Kasperowicz, Carbohyd. Res. 1986, 155, 252-257.

R. R. Schmidt, J. Michel, Angew. Chem. 1980, 92, 763-765; Angew. Chem. Int. Ed. Engl. 1980, 19, 731-732.

H. J. Schuster, Disseration, Göttingen 2008.

A. Speicher, J. Prakt. Chem. 2000, 342, 162-168.

J. C. Conway, P. Quayle, A. C. Reagan, C. J. Urch, Tetrahedron 2005, 61, 1191011923.

P. Renaud, A. Stojanovic, K. Schenk, Helv. Chim. Acta 1998, 81, 268-284.

H.-P. Wessel, T. Iversen, D. R. Bundle, J. Chem. Soc., Perkin Trans. 1 1985, 2247-2250.

S. Dietz, Dissertation, Göttingen 2011.

a) L. F. Tietze, N. Böhnke, S. Dietz, Org. Lett. 2009, 11, 2948-2950; b) L. F. Tietze, S. Dietz, N. Böhnke, M. A. Düfert, I. Objartel, D. Stalke, Eur. J. Org. Chem. 2011, 6574-6580.

H. Tanaka, A. Yoshozawa, T. Takahaschi, Angew. Chem. 2007, 119, 2557-2559.

Für eine Übersicht siehe: a) R. H. Grubbs, Tetrahedron 2004, 60, 7117-7140; b)

S. J. Connon, S. Blechert, Angew. Chem. Int. Ed. 2003, 42, 1900-1923; c) A. Fürstner, Angew. Chem. Int. Ed. 2000, 39, 3012-3043. 
a) T. Katsuki, K. B. Sharpless, J. Am. Chem. Soc. 1980, 102, 5974-5976; b) für eine Übersicht siehe: A. Pfenninger, Synthesis 1986, 2, 89-116.

70

C. M. König, K. Harms, U. Koert, Org. Lett. 2007, 9, 4777-4779.

D. Lutz, A. Güldner, R. Thums, P. Schreier, Tetrahedron: Asymmetry 1990, 1, 783-792.

G. Sabitha, B. Vangala, S. Siva Sankara Reddy, J. S. Yadav, Helv. Chim. Acta 2010, 93, 329-338.

R. Appel, Angew. Chem. Int. Ed. 1975, 14, 801-811.

Für eine Übersicht siehe: a) D. Seebach, Angew. Chem. 1979, 91, 259-278; b) B.-T. Gröbel, D. Seebach, Synthesis 1977, 6, 357-402.

A. B. Smith III, C. M. Adams, Acc. Chem. Res. 2004, 37, 365-377.

Für Beispiele siehe: a) D. S. Pisoni, D. B. Silva, R. A. Schenato, M. A. Ceschi, J. Braz. Chem. Soc. 2004, 14, 652-657; b) H. Takayanagi, Y. Kitano, Y. Morinaka, J. Org. Chem. 1994, 59, 2700-2706.
a) K. C. Nicolaou, X.-S. Peng, Y.-P. Sun, D. Polet, B. Zou, C. S. Lim, D. Y.-K. Chen, J. Am. Chem. Soc. 2009, 131, 10587-10597; b) M. E. Jung, B. A. Duclos, Tetrahedron 2006, 62, 9321-9334.

D. D. Perrin, W. L. F. Arnarego, Purification of Laboratory Chemicals, $3^{\text {rd }}$ Ed., Pergamon Press, Oxford, 1988. 


\section{Danksagung}

Wer glaubt, dass ein solch großes Unterfangen wie eine Doktorarbeit die Leistung einer einzelnen Person ist, der irrt sich. Um diese Aufgabe erfolgreich bestehen zu können, benötigt man neben dem chemischen Können und Wissen die Hilfe zahlreicher Leute, die einen auf verschiedene Art und Weise unterstützen.

Ein großer Dank gilt deshalb den analytischen Abteilungen des Instituts für Organische und Biomolekulare Chemie. Die Mitarbeiter der NMR-Abteilung Herr Reinhard Machinek, Frau Evelyn Pfeil, Frau Christiane Siebert, Herr Martin Weitemeyer und Frau Carola Zolke haben sowohl für die sorgfältige Aufnahme meiner NMR-Spektren als auch für die Erfüllung zahlreicher Sonderwünsche gesorgt. Auch bei den Mitarbeitern der Abteilung Massenspektrometrie Herrn Holm Frauendorf, Frau Gabriele Krökel, Frau Györgyi Sommer-Udvarnoki und Herrn Frank Hambloch bedanke ich mich für ihre stets freundliche, hilfsbereite Art und die Aufnahme meiner gefühlt unendlichen Zahl an Massenspektren. Besonders während der letzten Phase meiner Arbeit wart ihr eine große Unterstützung! Viel Arbeit hat mir auch Herr Olaf Senge abgenommen, indem er einen Großteil meiner ee-Werte per GC oder HPLC bestimmt hat.

Des Weiteren danke ich den Mitarbeitern der Chemikalienausgabe Herrn Rupert Schrommek und Herrn Holger Tucholla sowie allen Mitarbeitern der Werkstätten.

Unserer Sekretärin Frau Sabine Schacht möchte ich an dieser Stelle dafür danken, dass sie zu jeder Zeit den nötigen Durchblick im Chaos der Bürokratie hatte, Frau Martina Pretor danke ich für die Lösung meiner auftretenden PC-Probleme. Bei den Mitgliedern der Abteilung Tietze bedanke ich mich für die nette Arbeitsatmosphäre, besonderer Dank gilt dabei allen Weggefährten auf dem Gebiet der Spinosyne. Auch Daniel und seiner gesamten Abteilung danke ich für die vielen nützlichen Tipps und Ratschläge.

Für das sorgfältige Korrekturlesen dieser Arbeit danke ich Christoph „Heiko“ Eichhorst, Sven „Rapunzel“ Heidemann, Stefan Jackenkroll und Johannes Reiner 
sowie Tanja und Carolin Scheffer. Falls sich immer noch irgendwo Fehler eingeschlichen haben sollten, kann das auf jeden Fall nicht an euch gelegen haben!

Glücklicherweise bestand mein Leben in den letzten 8 1/2 Jahren nicht ausschließlich aus Chemie, Laboralltag und Uni. In dieser langen Zeit in Göttingen habe ich viele neue Freundschaften geschlossen und tolle, einzigartige Menschen kennengelernt. Die unzähligen lustigen Abende und auch Nächte werde ich niemals vergessen. Vielen Dank Alex, Annika, Arne, Claudia, Elli, Francesco, Frank, Frerk, Futti, Hannes, Julle, Jerome, Kiggi, Lennart, Nico, Stefan, Tilli, Wimbi oder wie ihr alle heißt. Aus dieser langen Liste möchte ich vor allem Arne, Frank, Futti und Julle hervorheben, mit denen ich seit Beginn meines Studiums durch Dick und Dünn gegangen bin. Ihr hattet auch immer Verständnis für meine kleinen Verspätungen. Auch meinen beiden langjährigen Mitbewohnern Miggen und Philipp (mein Arzt des Vertrauens) möchte ich danken. Ihr habt es mir wirklich leicht gemacht hier in Göttingen eine zweite Heimat zu finden.

Natürlich darf ich an dieser Stelle nicht meine Freunde aus der Heimat vergessen. Auch wenn wir uns nicht so häufig sehen, heißt das nicht, dass ihr mir nicht ebenso wichtig seid und dass euer Beitrag zum Gelingen dieser Arbeit nicht genauso groß war. Ihr hattet jederzeit ein offenes Ohr für meine Probleme und seid einfach großartig! Ich danke euch Dominik, Jordi, Jo, Anna, Joachim, Landi, Seppel, Seppi, Tommy und Trossi.

Mein größter Dank gilt allerdings meiner Familie ohne deren Unterstützung weder mein Studium noch diese Arbeit hier möglich gewesen wäre. Ihr habt mir in allen Lebenslagen Halt gegeben und mir immer gezeigt, dass ich auf dem richtigen Weg bin: Danke Mama \& Papa, Boris und Tanja! 
Meine akademischen Lehrer waren u.a. die folgenden Professoren und Dozenten:

G. Beuermann, P. Botschwina, M. Buback, A. de Meijere, U. Diederichsen, C. Ducho, L. Fitjer, H. Frauendorf, C. Griesinger, S. Grond, W. Hack, U. Klingebiel, H. Laatsch, T. Lenzer, R. Machinek, J. Magull, F. Meyer, C. Mösch-Zanetti, H. W. Roesky, J. Schroeder, C. Schulzke, G. M. Sheldrick, D. Stalke, C. Steinem, J. Stülke, M. Suhm, L. F. Tietze, J. Troe, S. Tsogoeva, D. B. Werz, A. Zeeck und P. von Zezschwitz. 


\section{Lebenslauf}

\section{Persönliche Angaben}

$\begin{array}{ll}\text { Name } & \text { Timo Scheffer } \\ \text { Geburtsdatum } & \text { 8. September } 1983 \\ \text { Geburtsort } & \text { Bad Hersfeld } \\ \text { Eltern } & \text { Werner Scheffer und Barbara Scheffer, geb. Michel } \\ \text { Nationalität } & \text { deutsch } \\ \text { Familienstand } & \text { ledig }\end{array}$

\section{Schulausbildung und Wehrdienst}

1989 bis 1993

1993 bis 2002

11. Juni 2002

$7 / 2002$ bis $3 / 2003$

\section{Hochschulausbildung}

$10 / 2003$

$10 / 2007$ bis $3 / 2008$

30. Juni 2008

$9 / 2008$ bis $2 / 2012$
Grundschule Asbach, Bad Hersfeld-Asbach

Wigbert Gymnasium Hünfeld, Hünfeld

Allgemeine Hochschulreife

Grundwehrdienst 2./ PzGrenBtl 152 in Schwarzenborn

Beginn des Chemiestudiums an der Georg-August-Universität Göttingen

Diplomarbeit am Institut für Organische und Biomolekulare Chemie der Georg-August-Universität Göttingen unter der Leitung von Prof. Dr. Dr. h.c. L. F. Tietze über das Thema "Untersuchungen zur Synthese von Spinosynanaloga"

Diplomprüfung im Studiengang Chemie Dissertation am Institut für Organische und Biomolekulare Chemie der Georg-August-Universität Göttingen unter der Leitung von Prof. Dr. Dr. h.c. Lutz F. Tietze über das Thema "Aufbau von Bausteinen zur Synthese von Spinosynanaloga" 